\title{
DRILLING BURR FORMATION WITH TOOL WEAR
}

by

Anna M. Mandra

Master of Engineering, Aerospace Engineering, Ryerson University (2010)

Bachelor of Engineering, Aerospace Engineering, Ryerson University (2007)

\author{
A dissertation \\ presented to Ryerson University \\ in partial fulfillment of the \\ requirements for the degree of \\ Doctor of Philosophy \\ in the program of \\ Aerospace Engineering
}

Toronto, Ontario, Canada, 2019

Copyright @Anna M. Mandra, 2019 


\section{AUTHOR'S DECLARATION FOR ELECTRONIC SUBMISSION OF A DISSERTATION}

I hereby declare that I am the sole author of this dissertation. This is a true copy of the dissertation, including any required final revisions, as accepted by my examiners.

I authorize Ryerson University to lend this dissertation to other institutions or individuals for the purpose of scholarly research.

I further authorize Ryerson University to reproduce this dissertation by photocopying or by other means, in total or in part, at the request of other institutions or individuals for the purpose of scholarly research.

I understand that my dissertation may be made electronically available to the public. 


\title{
DRILLING BURR FORMATION WITH TOOL WEAR
}

Anna M. Mandra, 2019

Doctor of Philosophy, Aerospace Engineering, Ryerson University, Toronto (2019)

\begin{abstract}
Automated assembly processes are being widely used in aircraft manufacturing as a hole must be drilled before fastener installation. Burr formation is of importance for machining processes including drilling. A burr, an unwanted by-product of machining, is a raised edge that remains attached to a workpiece because of the loss of shear action. In drilling, burrs form as the drill enters and exits the part, particularly when the tool becomes worn. Burrs require an additional process to deburr, incurring extra cost and time.
\end{abstract}

To understand drilling burr formation, this dissertation presents a new model that combines a burr model with a tool-wear model. The first adopted model describes a burr with respect to its height and thickness and relates burr formation to the drilling thrust force generated from drilling with a sharp tool. This model comprises a number of key factors: part material property, tool geometry, and drilling parameters. In reality, these factors will vary. Preliminary work was done to use this model to develop a sensitivity matrix for the purpose of studying the effect of variation in the key factors on burr formation. Unfortunately, tool wear, which is a major issue, cannot be simply considered as a variation in tool geometry. When a tool becomes worn, the cutting edge radius becomes large, and the cutting action changes from shearing to ploughing.

A second model adopted is a tool wear model, leading to the core work of this dissertation. The aim of this study is to develop a drilling burr-formation model for realistic conditions, incorporating variance within the tool and process parameters. The proposed method applies a weighting model that can 
consider the wear/no-wear condition for each segment, thereby leading to a hybrid model for drilling burr formation. The research method is validated through simulation and an experiment entailing the generation of drilling burrs in conditions mimicking those in aerospace manufacturing. The results show that the model successfully estimates burr height and thickness trends with increasing levels of tool wear, for penetrated holes. The methods and results presented in this dissertation show good promise for understanding the effects of tool wear on drilling burr growth. 


\section{ACKNOWLEDGEMENTS}

First, I would like to thank my supervisor Dr. Fengfeng Xi, without whose support, encouragement and guidance this thesis would not have been possible. His comprehensive knowledge of machining and manufacturing was instrumental to what I have accomplished. I would also like to thank my cosupervisor Dr. Puren Ouyang, for his direction and helpful feedback during the course of this work. Thank you.

I wish to express my gratitude to the defense committee consisting of Dr. O. Remus Tutunea-Fatan from Western University, Dr. Seyed Hashemi and Dr. Bo Tan from the Department of Aerospace Engineering, and Dr. Ahmad Ghasempoor from the Department of Mechanical Engineering. Their insightful comments greatly improved the quality of this dissertation. I would like to thank Dr. Jeffrey Yokota, Graduate Program Director for the Department of Aerospace Engineering. Dr. Yokota's enthusiasm and inspiration when I was contemplating graduate studies when I was still an undergraduate student and throughout graduate school, got me to where I am today.

I would also like to thank Ryerson University for the various graduate scholarships and awards which helped support this research.

Finally I would like to thank my dear parents and my amazing friends. You were always there for me with support, understanding and constant encouragement. Thank you. 


\section{TABLE OF CONTENTS}

AUTHOR'S DECLARATION FOR ELECTRONIC SUBMISSION OF A DISSERTATION .........................................ii

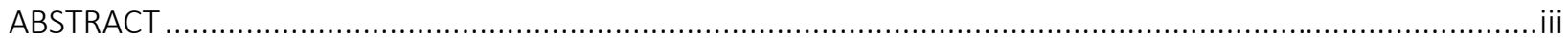

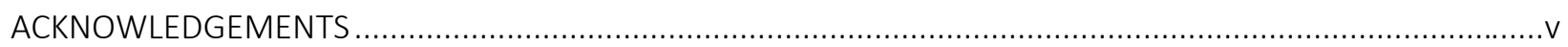

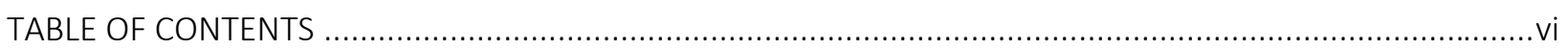

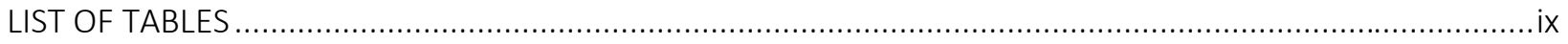

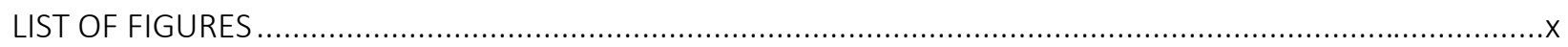

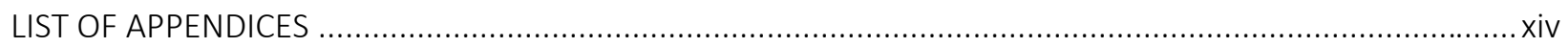

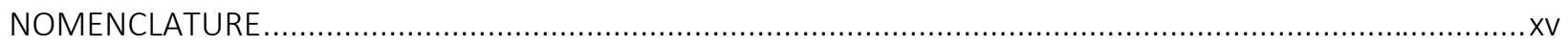

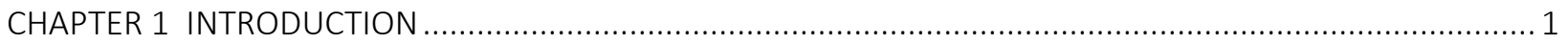

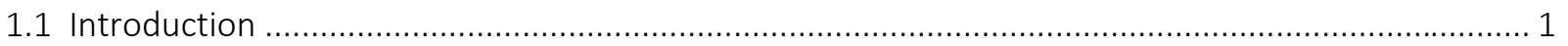

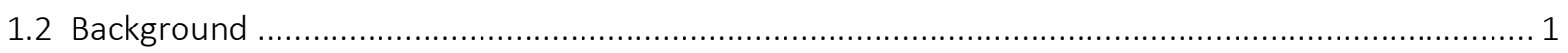

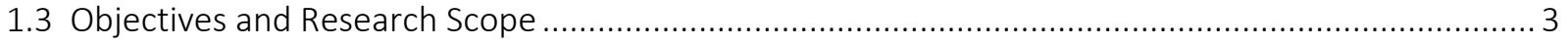

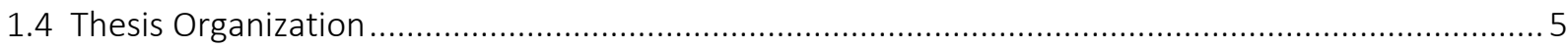

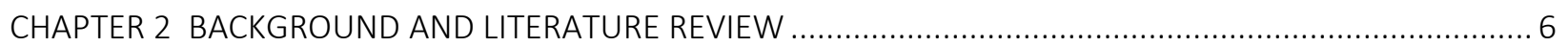

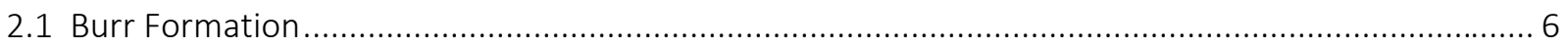

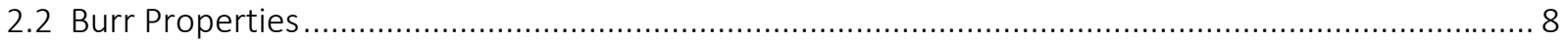

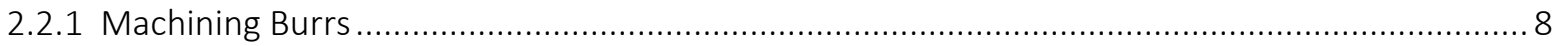

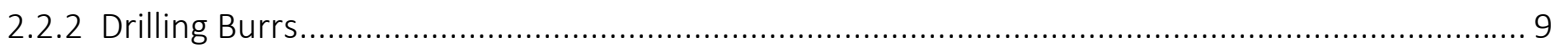

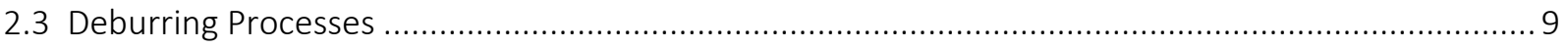

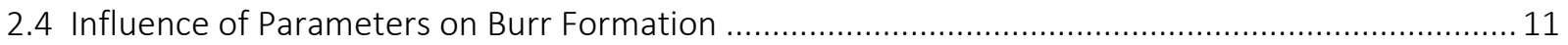

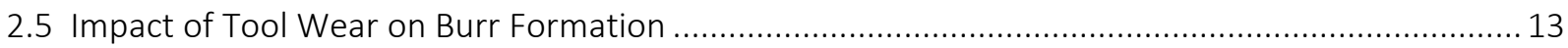

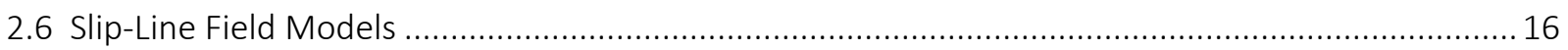

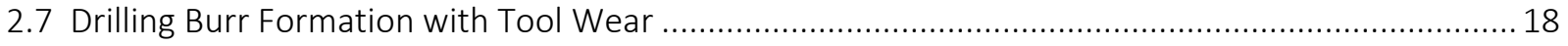

CHAPTER 3 DRILLING BURR FORMATION AND BURR SENSITIVITY ANALYSIS ...................................... 20

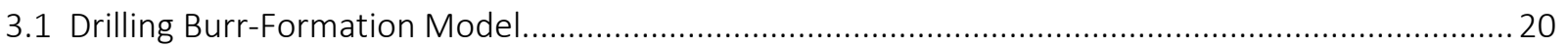

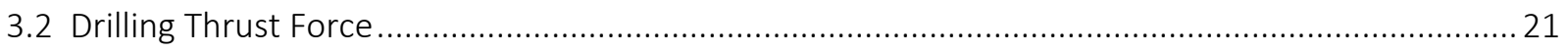

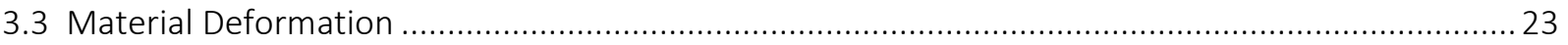

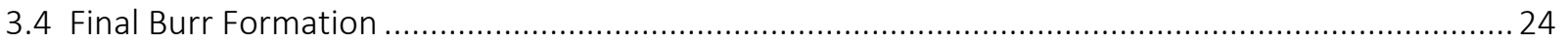

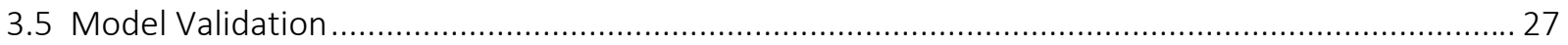




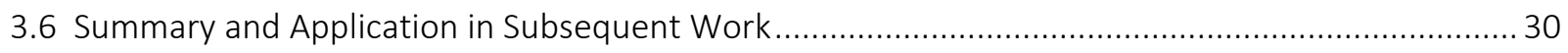

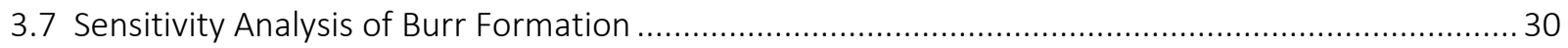

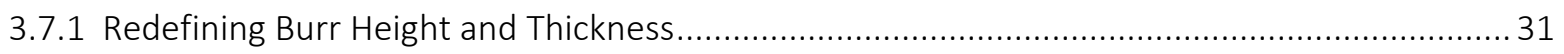

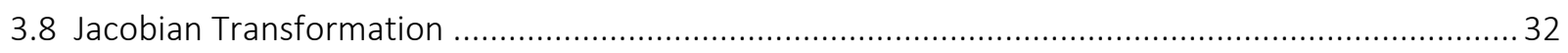

3.8.1 Jacobian Transformation Applied to Burr Height ................................................................. 33

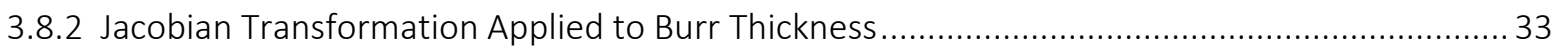

3.8.3 Continued Derivation of Burr-Height and Thickness Relationships ....................................... 34

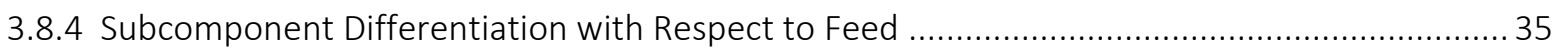

3.8.5 Subcomponent Differentiation with Respect to Point Angle ................................................ 36

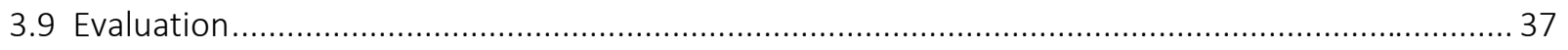

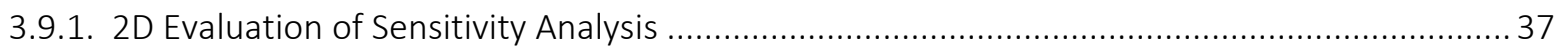

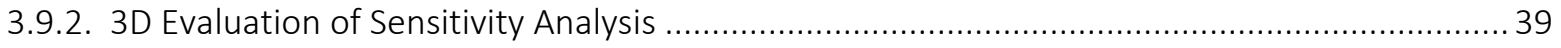

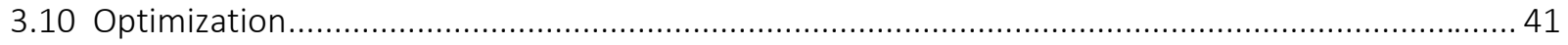

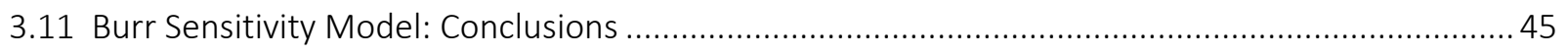

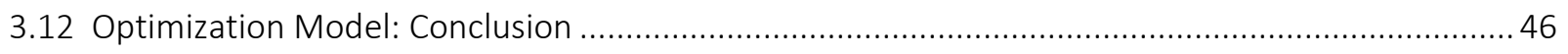

CHAPTER 4 PLOUGHING FORCE ANALYSIS AND NEW BURR FORMATION MODEL ................................ 49

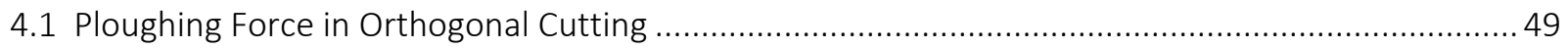

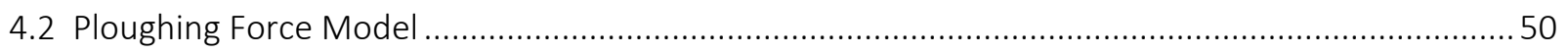

4.3 New Burr Formation Model: Combining Ploughing and Drilling Thrust Forces............................. 52

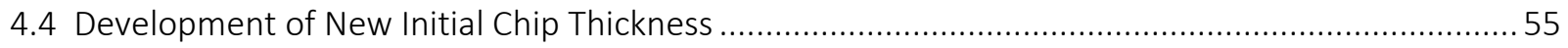

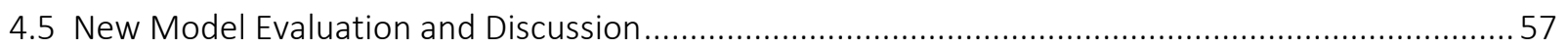

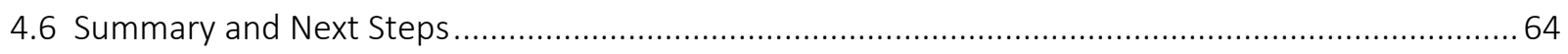

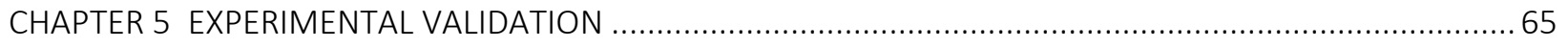

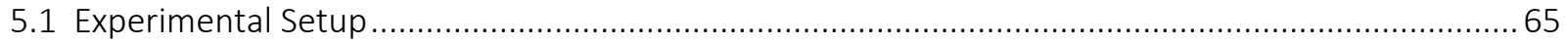

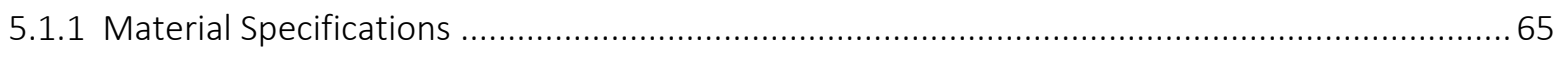

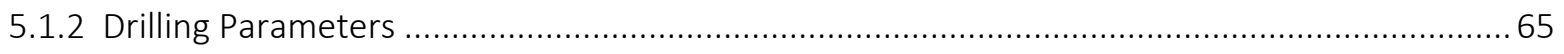

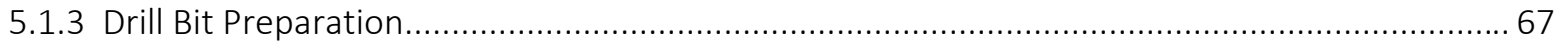

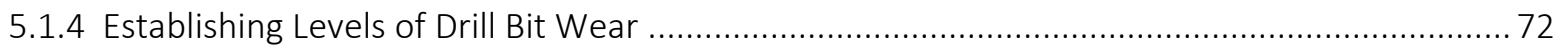

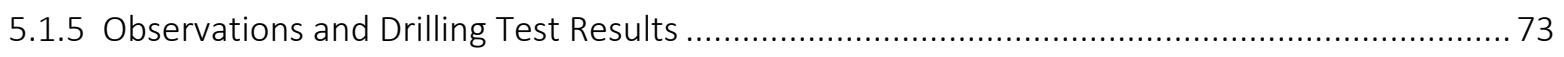

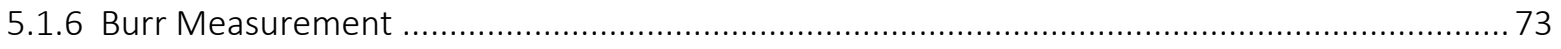

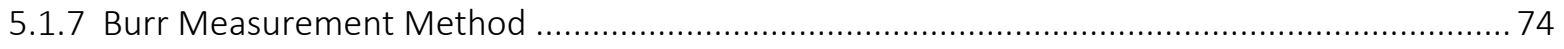

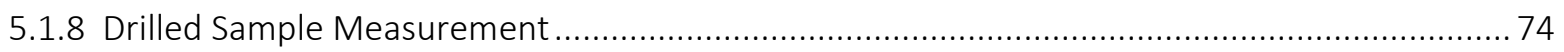




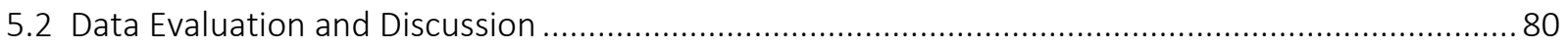

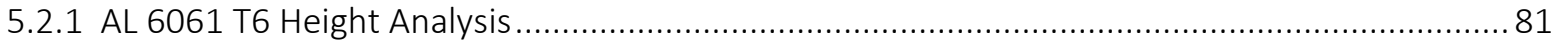

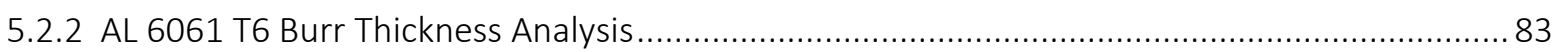

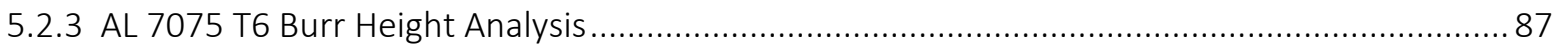

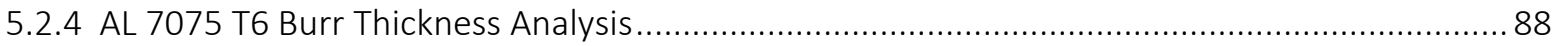

5.3 Additional Considerations Regarding Experimental Data .......................................................... 93

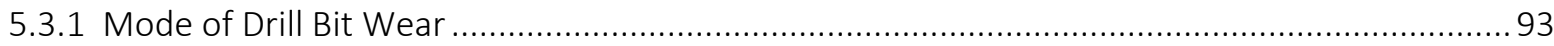

5.3.2 Modeling for Penetrated vs. Non-Penetrated Holes ...................................................... 94

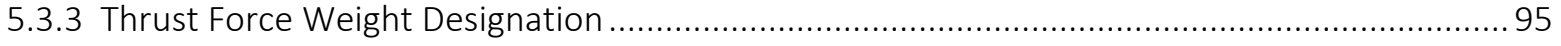

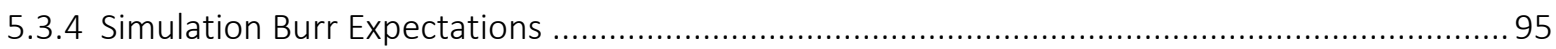

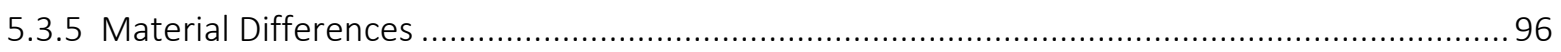

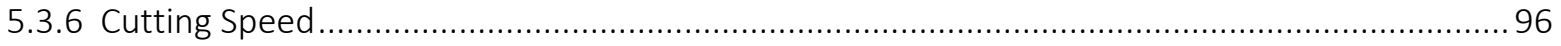

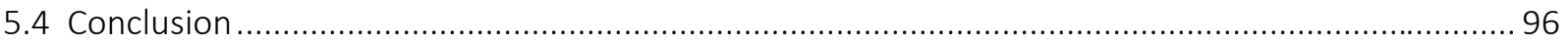

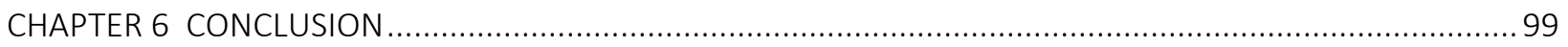

6.1 Summary of Proposed Mode and Research Conclusions ............................................................. 99

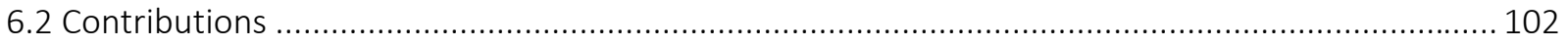

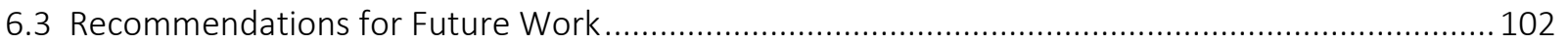

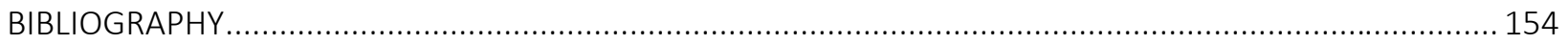




\section{LIST OF TABLES}

Table 2.1 Three common drilling burrs and associated drilling burr formation mechanisms.................... 9

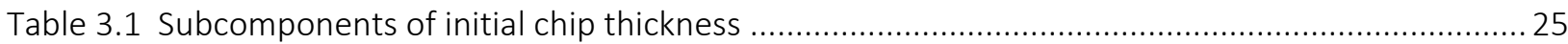

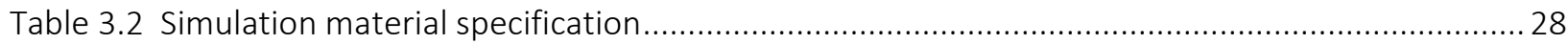

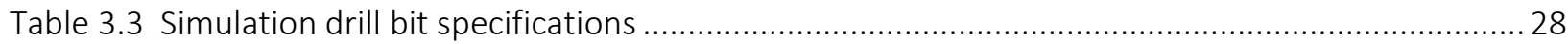

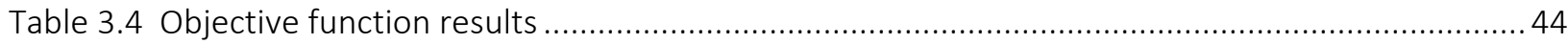

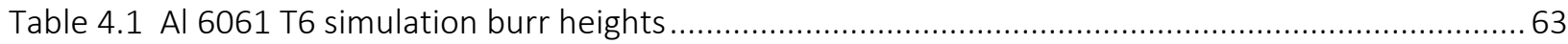

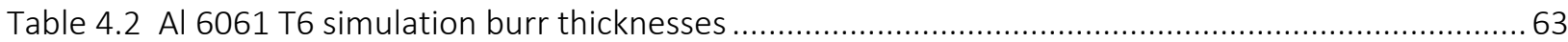

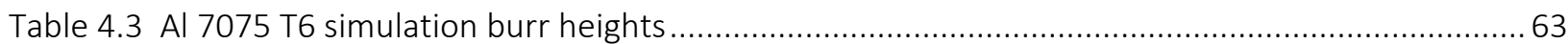

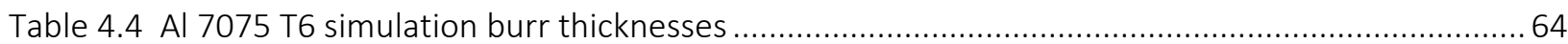

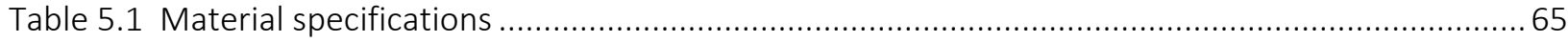

Table 5.2 Drill bit edge radii measurements and wear calculation ..................................................... 72

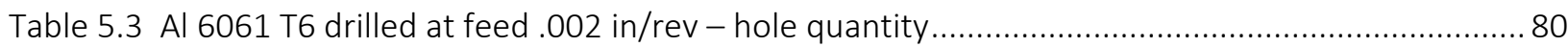

Table D.1 Objective function results, continued …............................................................................ 133

Table G.1 Al 6061 T6 burr height data at feed .002 in/rev............................................................. 142

Table G.2 Al 6061 T6 burr height data at feed .004 in/rev............................................................ 143

Table G.3 Al 6061 T6 burr height data at feed .006 in/rev............................................................ 144

Table G.4 Al 6061 T6 burr thickness data at feed .002 in/rev ............................................................... 145

Table G.5 Al 6061 T6 burr thickness data at feed .004 in/rev ............................................................. 146

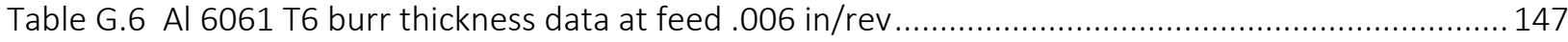

Table G.7 Al 7075 T6 burr height data at feed .002 in/rev............................................................... 148

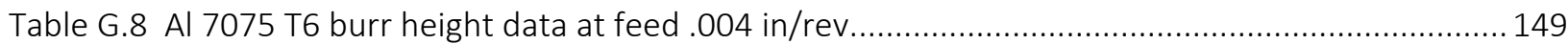

Table G.9 Al 7075 T6 burr height data at feed .006 in/rev.............................................................. 150

Table G.10 Al 7075 T6 burr thickness data at feed .002 in/rev ........................................................... 151

Table G.11 Al 7075 T6 burr thickness data at feed .004 in/rev ....................................................... 152

Table G.12 Al 7075 T6 burr thickness data at feed .006 in/rev .......................................................... 153 


\section{LIST OF FIGURES}

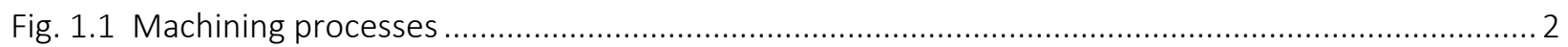

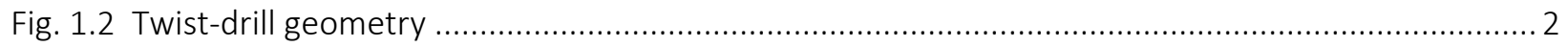

Fig. 1.3 Tool with a sharp cutting edge in orthogonal cutting, adapted from [10, Fig. 1.1] ...................... 3

Fig. 1.4 Orthogonal cutting with a worn tool, adapted from [10, Fig. 2.4.1] .......................................... 4

Fig. 2.1 Four stages of burr formation, adapted from [22, Fig. 30] ......................................................... 6

Fig. 2.2 Slip-line field for wedge sliding, adapted from [15, Fig. 2], [39] .................................................. 17

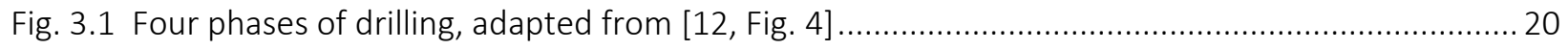

Fig. 3.2 Orthogonal-cutting model and Merchant-circle model ............................................................ 22

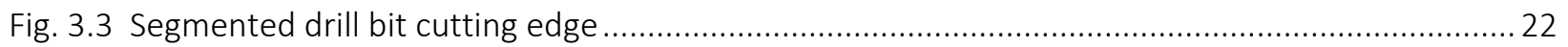

Fig. 3.4 Graphical depiction of material deformation in drilling .............................................................. 24

Fig. 3.5 Half cross section of material prior to and after deformation, adapted from [12, Fig. 6] ............ 24

Fig. 3.6 Burr height and thickness formation, adapted from [12, Fig. 7] .............................................. 27

Fig. 3.7 Burr height values variance with increasing N-value of segments of AISI 304L, drilled with 3.968

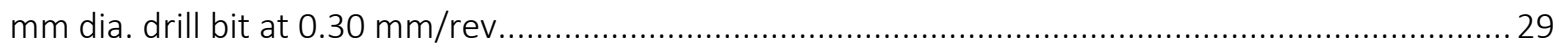

Fig. 3.8 Burr height and rate of change of burr height for AISI 304L, 1.984-mm dia. drill bit .................. 38

Fig. 3.9 Burr thickness and rate of change of burr thickness for AISI 304L, 1.984-mm dia. drill bit .......... 39

Fig. 3.10 AISI 304L 1.984-mm dia. - surface plots of burr height, thickness, and respective rates of change of height and thickness vs. point angle and feed

Fig. 3.11 Sample objective function 1 - Material: AISI 304L, drill bit dia. $1.984 \mathrm{~mm}$.............................. 44

Fig. 3.12 Sample objective function 1 - Material: AISI 304L, drill bit dia. 3.968 mm ............................ 44

Fig. 3.13 Sample objective function 1 - Material: AISI 4118, drill bit dia. $4 \mathrm{~mm}$.................................... 44

Fig. 3.14 Sample objective function 1 - Material: AISI 4118, drill bit dia. $7 \mathrm{~mm}$.................................. 45

Fig. 4.1 Slip-line field model for ploughing in orthogonal cutting, adapted from [15, Fig. 3] ...................51

Fig. $4.2(\mathrm{a}-\mathrm{c})$ Calculated total drilling thrust force [N] in relation to drill bit wear [\%] for Al 6061 T6. The

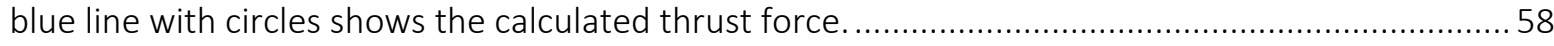

Fig. $4.3(a-c)$ Calculated total drilling thrust force [N] in relation to drill bit wear [\%] for Al 7075 T6. The blue line with circles shows the calculated thrust force. 59

Fig. $4.4(\mathrm{a}-\mathrm{c})$ Calculated initial thickness of deforming material t0 [ $\mu \mathrm{m}]$ in relation to drill bit wear [\%] for Al 6061 T6. The blue line with circles shows the calculated t0. 60 
Fig. $4.5(\mathrm{a}-\mathrm{c})$ Calculated initial thickness of deforming material t0 [ $\mu \mathrm{m}]$ in relation to drill bit wear [\%] for Al 7075 T6. The blue line with circles shows the calculated t0. 61

Fig. 5.1 Drilling test article drawing 67

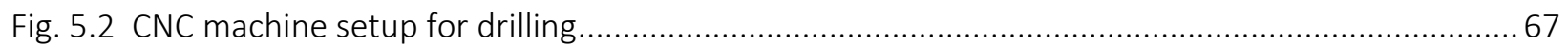

Fig. 5.3 Dremel tool with flat grinding disk used for drill bit wear down .............................................. 68

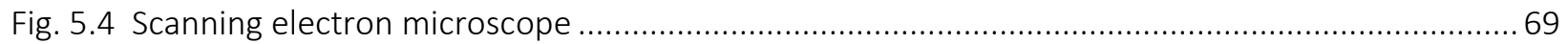

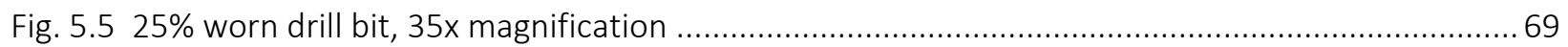

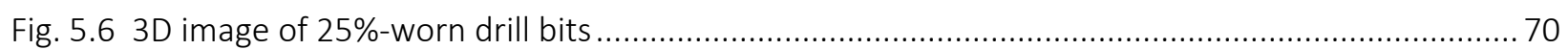

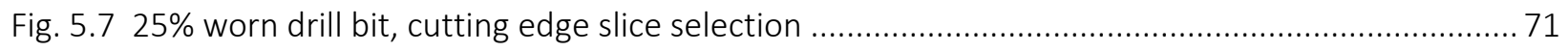

Fig. 5.8 25\% worn drill bit, edge radius measurement...................................................................... 71

Fig. 5.9 Burr generated from an Al 6061 T6, .090"-thick plate, drilled with a 50\% worn drill bit at .002 in/rev (sample $1-\mathrm{L})$. .74

Fig. 5.10 Burr height measurement of burr generated from a plate of Al 6061 T6, .090" thick, drilled with

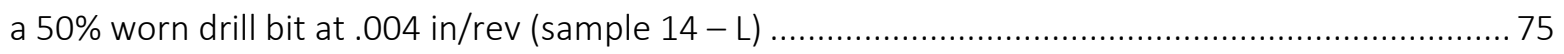

Fig. 5.11 Burr height measurement of burr generated from a plate of Al 6061 T6, .040" thick, drilled with

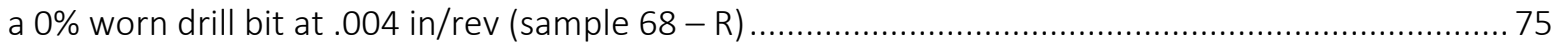

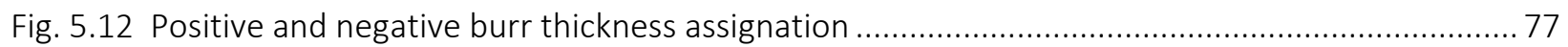

Fig. 5.13 Burr thickness measurement of burr generated from drilling a Al 6061 T6, .090" thick plate, with

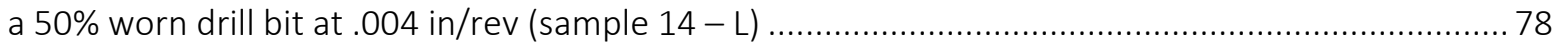

Fig. 5.14 Burr thickness measurement of burr generated from drilling a Al 6061 T6, .040" thick plate, with a $50 \%$ worn drill bit at .004 in/rev (sample $9-\mathrm{L}$ ) 78

Fig. 5.15 Burr thickness, top view of burr generated from drilling a Al 6061 T6, .090" thick plate with a $75 \%$ worn drill bit at .006 in/rev (sample $40-\mathrm{L}$ )

Fig. 5.16 Burr thickness, top view of burr generated from drilling a Al 6061 T6, .090" thick plate with a $75 \%$ worn drill bit at .006 in/rev (sample $40-$ L) . . . 79

Fig. $5.17(\mathrm{a}-\mathrm{c})$ Burr height [ $\mu \mathrm{m}]$ in relation to drill-bit wear [\%] resulting from drilling Al 6061 T6. The red error bars represent burr heights drilled in .090" thick sheets, the green error bars represent burr heights drilled in .040" thick sheets, and the blue line with circles shows the calculated burr heights.

Fig. 5.18 (a-c) Burr thickness [ $\mu \mathrm{m}$ ] in relation to drill-bit wear [\%] resulting from drilling Al 6061 T6. The red error bars represent burr heights drilled in .090" thick sheets, the green error bars represent 
burr heights drilled in .040" thick sheets, and the blue line with circles shows calculated burr heights. 86

Fig. 5.19 (a-c) Burr height [ $\mu \mathrm{m}]$ in relation to drill-bit wear [\%] resulting from drilling Al 7075 T6. The red error bars represent burr heights drilled in .090" thick sheets, the green error bars represent burr heights drilled in .040" thick sheets, and the blue line with circles shows calculated burr heights.... 91

Fig. $5.20(a-c)$ Burr thickness [ $\mu \mathrm{m}$ ] in relation to drill-bit wear [\%] resulting from drilling Al 7075 T6. The red error bars represent burr heights drilled in .090" thick sheets, the green error bars represent burr heights drilled in .040" thick sheets, and the blue line with circles shows the calculated burr heights. 92

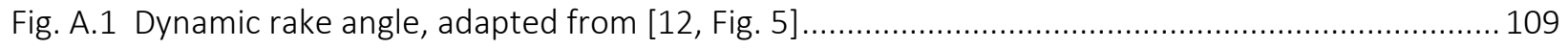

Fig. A.2 Burr Formation Model for Sharp Tools Flowchart.................................................................. 113

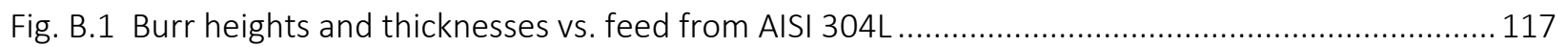

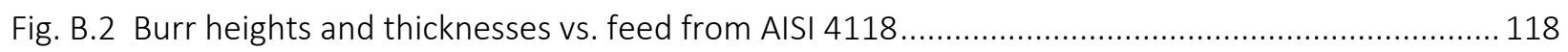

Fig. B.3 Burr heights and thicknesses vs. point angle from AISI 304L ................................................ 119

Fig. B.4 Burr heights and thicknesses vs. point angle from AISI 4118 ............................................... 120

Fig. D.1 Burr height and rate of change of burr height for AISI 304L, 3.968 mm dia. drill bit ................ 124

Fig. D.2 Burr thickness and rate of change of burr thickness for AISI 304L, 3.968mm dia. drill bit .......... 125

Fig. D.3 Burr height and rate of change of burr height for AISI 4118, 4 mm dia. drill bit....................... 126

Fig. D.4 Burr thickness and rate of change of burr thickness for AISI 4118, 4 mm dia. drill bit ............... 127

Fig. D.5 Burr height and rate of change of burr height for AISI 4118, $7 \mathrm{~mm}$ dia. drill bit...................... 128

Fig. D.6 Burr thickness and rate of change of burr thickness for AISI 4118, 7 mm dia. drill bit .............. 129

Fig. D.7 AISI 304L dia. 3.968mm - surface plots of burr height, thickness, and respective rates of change

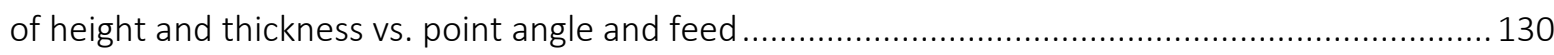

Fig. D.8 AISI 4118 dia. $4 \mathrm{~mm}$ - surface plots of burr height, thickness, and respective rates of change of height and thickness vs. point angle and feed 131

Fig. D.9 AISI 4118 dia. $7 \mathrm{~mm}$ - surface plots of burr height, thickness, and respective rates of change of

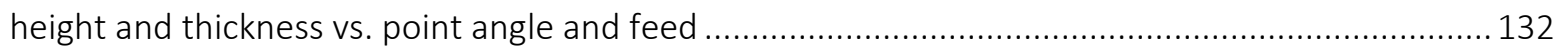

Fig. D.10 Sample objective function 2 - Material: AISI 304L, drill bit dia. $1.984 \mathrm{~mm}$............................ 133

Fig. D.11 Sample objective function 2 - Material: AISI 304L, drill bit dia. $3.968 \mathrm{~mm}$............................ 133

Fig. D.12 Sample objective function 2 - Material: AISI 4118, drill bit dia. 4 mm .................................... 133

Fig. D.13 Sample objective function 2 - Material: AISI 4118, drill bit dia. 7 mm .................................. 134

Fig. D.14 Sample objective function 3 - Material: AISI 304L, drill bit dia. 1.984 mm ............................ 134 
Fig. D.15 Sample objective function 3 - Material: AISI 304L, drill bit dia. 3.968 mm .

Fig. D.16 Sample objective function 3 - Material: AISI 4118, drill bit dia. 4 mm ................................... 135

Fig. D.17 Sample objective function 3 - Material: AISI 4118, drill bit dia. 7 mm ................................... 135

Fig. F.1 Ploughing force and new combined drilling thrust force model flowchart............................... 141 


\section{LIST OF APPENDICES}

APPENDIX A: DRILLING BURR FORMATION MODEL FOR SHARP TOOLS AND ORTHOGONAL CUTTING DESCRIPTION 106

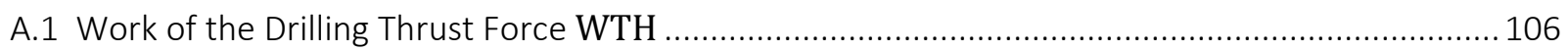

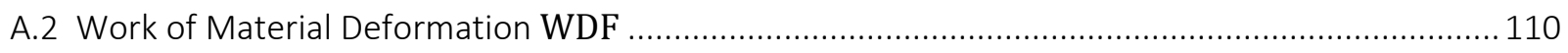

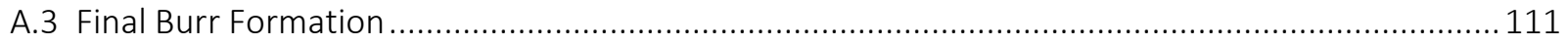

A.3.1 Burr Formation Model for Sharp Tools Flowchart …......................................................... 113

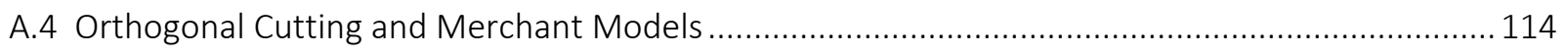

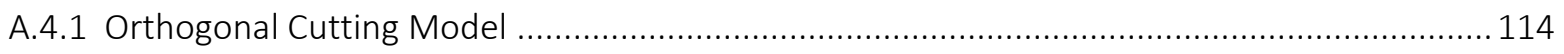

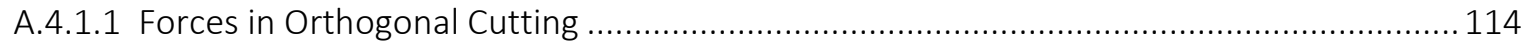

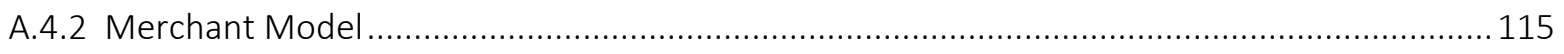

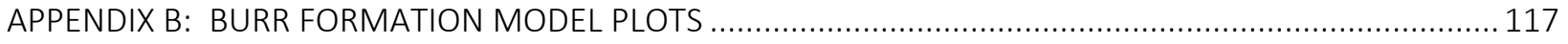

B.1 Burr Heights and Thicknesses with Respect to a Range of Feeds .............................................. 117

B.2 Burr Heights and Thicknesses with Respect to a Range of Point Angles ...................................... 119 APPENDIX C: BURR SENSITIVITY MODEL - DIFFERENTIATION OF $\Phi$ WITH RESPECT TO FEED $\partial \Phi \partial \mathrm{f}$ AND

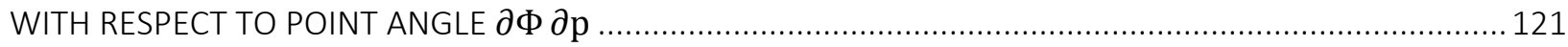

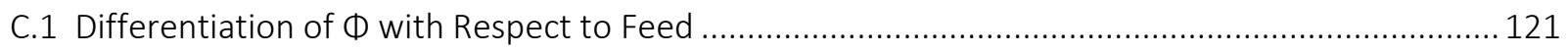

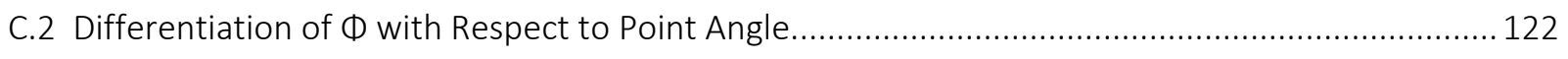

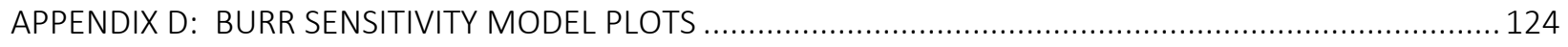

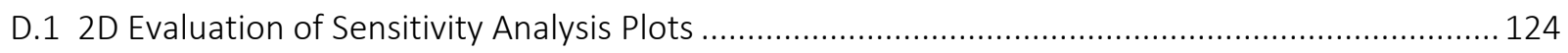

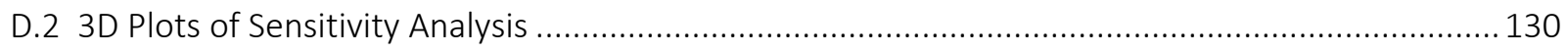

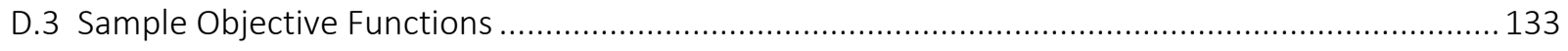

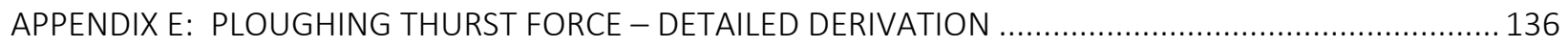

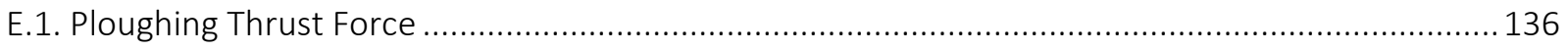

APPENDIX F: PLOUGHING FORCE AND NEW COMBINED DRILLING THRUST FORCE MODEL FLOWCHART 139

APPENDIX G: EXPERIMENTAL DATA FROM DRILLING AL 6061 T6 AND AL 7075 T6 …........................... 142

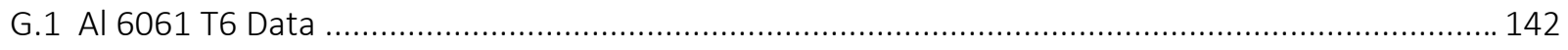

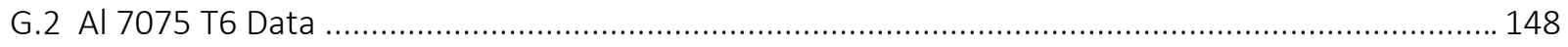




\section{NOMENCLATURE}

\begin{tabular}{|c|c|}
\hline$\alpha_{d}$ & dynamic rake angle \\
\hline$\alpha_{n}$ & normal rake angle \\
\hline$A_{S}$ & shear plane area \\
\hline$\beta$ & friction angle \\
\hline$C A$ & $\begin{array}{l}\text { region of the ploughing model, representing the workpiece and the bottom } \\
\text { surface of the material buildup region }\end{array}$ \\
\hline$e$ & efficiency in the objective function \\
\hline$f$ & feed \\
\hline $\mathcal{F}$ & objective function \\
\hline$F_{p l}$ & ploughing thrust force \\
\hline$\Delta F_{p l}$ & ploughing thrust force on an individual cutting edge segment \\
\hline$F_{S}$ & shear force \\
\hline$F_{T H}$ & drilling thrust force \\
\hline$\Delta F_{T H}$ & drilling thrust force acting on an individual cutting edge segment \\
\hline$\Delta F_{T H-T O T A L}$ & new proposed drilling thrust force \\
\hline$H$ & burr height \\
\hline$H_{1}$ & $\begin{array}{l}\text { subcomponent of burr height, representing } t_{0} \text {, in preparation for a Jacobian } \\
\text { transformation }\end{array}$ \\
\hline $\mathrm{H}_{2}$ & $\begin{array}{l}\text { subcomponent of burr height, representing } \sin p \exp \left\{\frac{\sqrt{3}}{2} \ln \left(\frac{100}{100-\% R \cdot A .}\right)\right\} \text { in } \\
\text { preparation for a Jacobian transformation }\end{array}$ \\
\hline$i$ & subscript referring to the individual element along the drill bit cutting edge \\
\hline $\mathcal{J}$ & Jacobian matrix \\
\hline$J_{1}, J_{2}$, and $J_{3}$ & subcomponents of $t_{0}$ \\
\hline$k$ & shear strength of the workpiece in the ploughing force model \\
\hline$L$ & total distance across the drill bit cutting edge \\
\hline$m$ & friction factor \\
\hline$M_{b}$ & bending moment \\
\hline$N$ & number of segments along the segmented drill bit cutting edge \\
\hline$\eta$ & angle between slip line and the bottom surface of the built-up region \\
\hline$\Delta \theta$ & angle of rotation of bending deformation \\
\hline$p$ & drill half point angle \\
\hline$\rho$ & relative radius \\
\hline$\rho_{\text {row }}$ & prow angle \\
\hline$\sigma_{y}$ & tensile yield strength \\
\hline$\sigma_{u}$ & ultimate strength \\
\hline
\end{tabular}




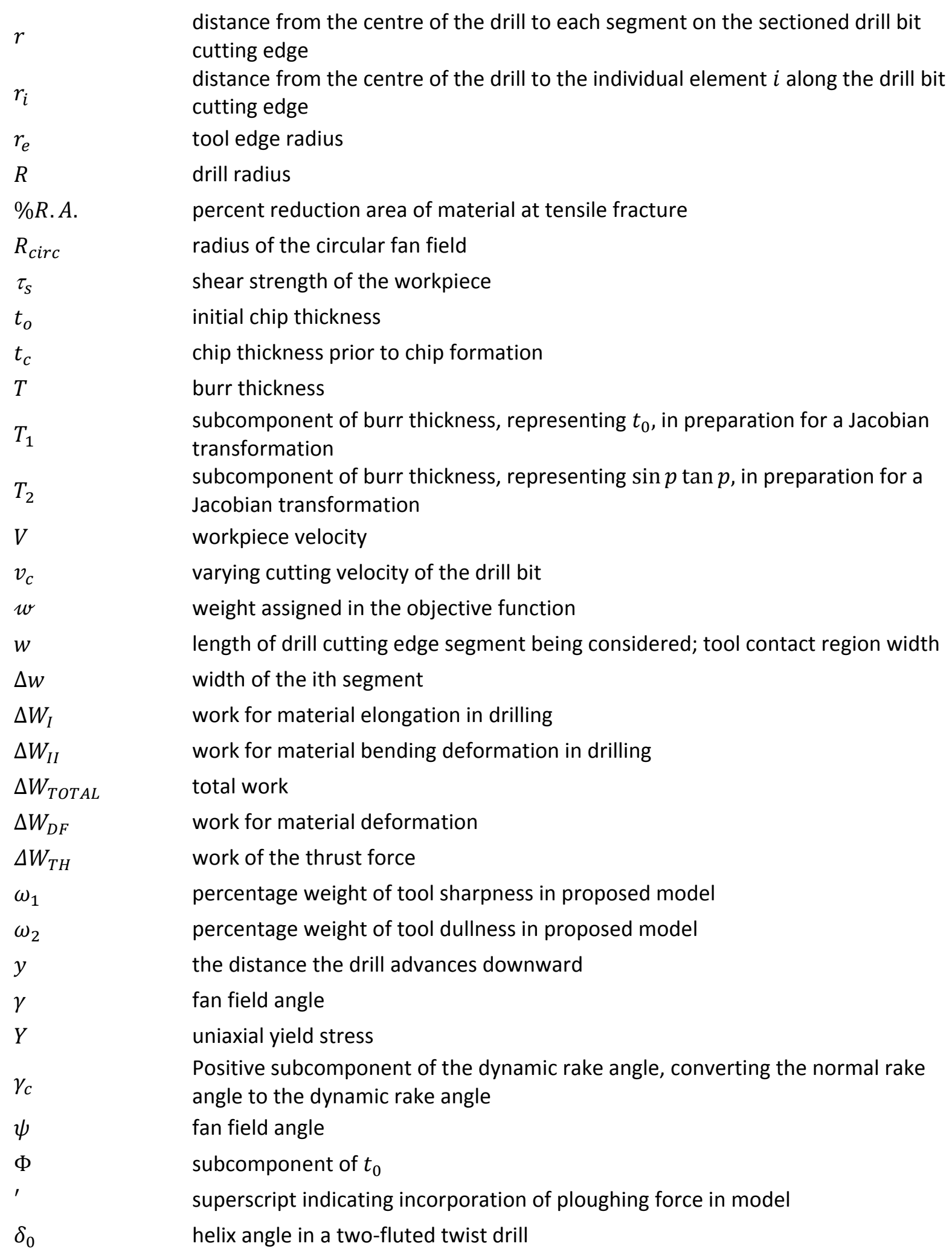

$r$

$r_{i}$

$r_{e}$

$R$

$\% R$. A.

$R_{\text {circ }}$

$\tau_{S}$

$t_{o}$

$t_{c}$

$T$

$T_{1}$

$T_{2}$

V

$v_{c}$

w

w

$\Delta w$

$\Delta W_{I}$

$\Delta W_{I I}$

$\Delta W_{\text {TOTAL }}$

$\triangle W_{D F}$

$\Delta W_{T H}$

$\omega_{1}$

$\omega_{2}$

$y$

$\gamma$

Y

$\gamma_{c}$

$\psi$

$\Phi$

,

$\delta_{0}$

distance from the centre of the drill to each segment on the sectioned drill bit cutting edge

distance from the centre of the drill to the individual element $i$ along the drill bit cutting edge

tool edge radius

drill radius

percent reduction area of material at tensile fracture

radius of the circular fan field

shear strength of the workpiece

initial chip thickness

chip thickness prior to chip formation

burr thickness

subcomponent of burr thickness, representing $t_{0}$, in preparation for a Jacobian transformation

subcomponent of burr thickness, representing $\sin p \tan p$, in preparation for a Jacobian transformation

workpiece velocity

varying cutting velocity of the drill bit

weight assigned in the objective function

length of drill cutting edge segment being considered; tool contact region width width of the ith segment

work for material elongation in drilling

work for material bending deformation in drilling

total work

work for material deformation

work of the thrust force

percentage weight of tool sharpness in proposed model

percentage weight of tool dullness in proposed model

the distance the drill advances downward

fan field angle

uniaxial yield stress

Positive subcomponent of the dynamic rake angle, converting the normal rake angle to the dynamic rake angle

fan field angle

subcomponent of $t_{0}$

superscript indicating incorporation of ploughing force in model

helix angle in a two-fluted twist drill 


\section{CHAPTER 1 INTRODUCTION}

\subsection{Introduction}

Drilling is one of the most common machining processes in aerospace manufacturing used for fastening and riveting purposes in parts and large components final assembly. Each drilled hole creates drilling burrs, which are both prevalent and problematic in aerospace manufacturing. The number of mechanical fasteners in a typical fighter aircraft can be in the range of $200,000-300,000$, while a commercial airliner or transport aircraft can have as many as 1.5-3 million, depending on aircraft size [1]. The manufacturing process of Airbus wings involves drilling upwards of 40 million holes in aircraft structures per annum, the majority of which (80\%) are drilled manually. The assembly involves the joining of spars, ribs, and stringers, which, in an Airbus A380, support 20 individual skin panels [2]. The assembly of the fuselage panel is similarly work intensive [3]. Burr removal can amount to up to $30 \%$ of the cost of a precision aerospace part and $14 \%$ of an automotive component [4]. Burr formation in drilling and its contributing factors are, therefore, a critical topic currently as airplanes continue to be assembled with rivets and fasteners that require drilled holes. First-time right practices along with cost saving measures have always been key topics for aerospace manufacturers. Burr formation is a consistent and persistent problem in aerospace manufacturing that requires additional research as there is not yet an optimal solution. This need is coupled with the modern manufacturing trend of planning for burr minimization and prevention, instead of resorting to costly removal processes [5].

\subsection{Background}

A burr, as defined by the Society of Manufacturing Engineers, is an undesirable projection of material resulting from a cutting or shearing operation [6]. It is also defined as an unwanted by-product of machining, in the form of a raised edge, created because of the loss of shear action, that remains attached to a workpiece [7]. Burr formation increases the component rejection rate in the final production stage, causing high resource wastage [8]. Burrs increase the stress concentration at hole edges, which already have three times the net section stress. Burrs can also interfere with the proper fitting of mechanical fasteners. Removing burrs diminishes stress concentration, increasing fracture resistance and fatigue life. It reduces damage to fasteners and to clamped components during assembly [9]. 
Milling, drilling, and turning are essential processes for the majority of large-scale manufacturing operations, as portrayed in Fig.. Drilling is a main component of these machining processes, comprising about $25 \%$ of total machining. Drilling is also a major operation in aerospace part manufacturing and assembly; aerospace assembly can represent up to $50 \%$ of the total delivered aircraft cost [1], [4].

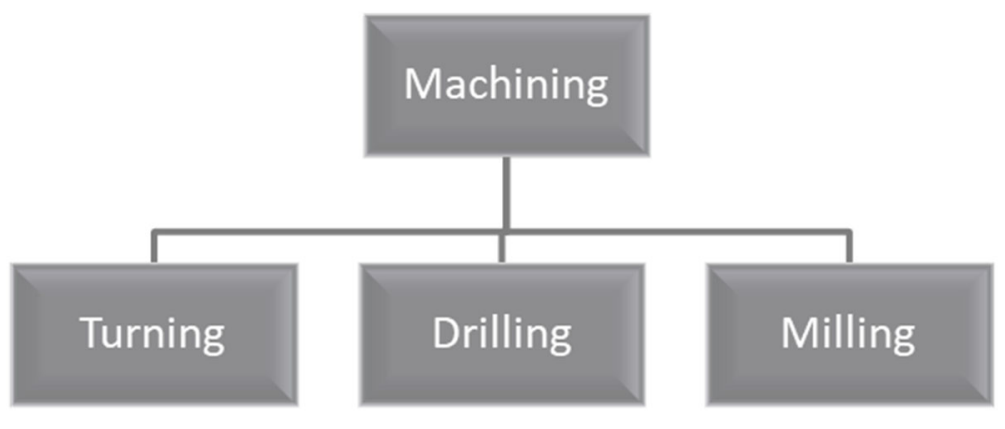

Fig. 1.1 Machining processes

Drilling quality is affected by tool geometry and process parameters. The most common drill used is the standard-point twist drill. Its main parameters include a point angle, helix angle, drill diameter, chisel edge, cutting edge, and diameter, as shown in Fig. 1.2. In practice, drill geometry changes with tool wear.

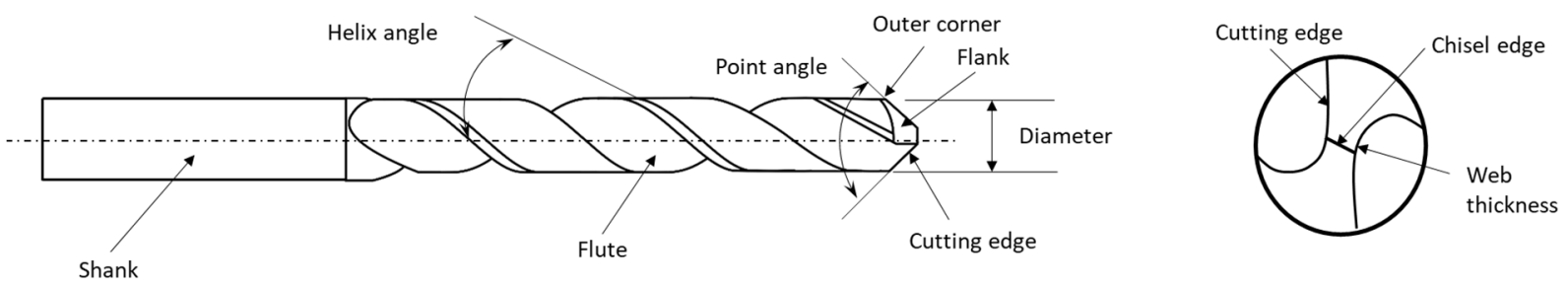

Fig. 1.2 Twist-drill geometry

The importance of arriving at a drilling and burr formation model that minimizes burr, reduces the need for deburring, eliminates waste, and contributes to cost savings, is clear. A significant restriction in most machining and drilling burr formation models is the assumption of an ideal, sharp cutting tool with an infinitely small edge radius, as shown in Fig. 1.3 [10], together with unvarying process conditions. A comparison to orthogonal cutting models is warranted because orthogonal cutting is the foundation of most machining processes. Sharp tools with ideal process parameters do not represent day-to-day drilling conditions, where wear begins the moment drilling begins, even with a brand new drill bit. Understanding burr-affecting conditions helps burr prediction. Burr prediction is important for process 
planning and optimization, including machining and deburring. Optimal process planning can eventually lead to burr reduction by as much as $70 \%$.

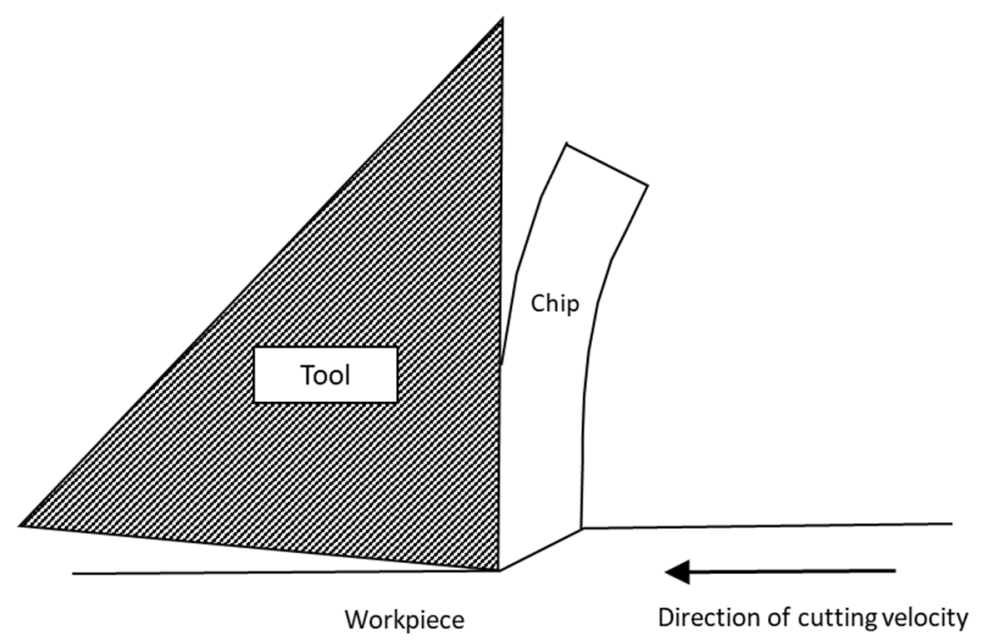

Fig. 1.3 Tool with a sharp cutting edge in orthogonal cutting, adapted from [10, Fig. 1.1]

\subsection{Objectives and Research Scope}

In general, drilling burr formation is a multifaceted mechanism, affected by tool geometry, process parameters, and material properties, all of which may vary. Through a range of studies, past and ongoing, it has been established that factors most affecting drill burr height and thickness are process conditions, type of material, and drill bit geometry [8], [11] - [14].

Following are the objectives of this dissertation:

i. Develop a drilling burr formation model for realistic conditions, incorporating variance in the tool and process parameters;

ii. Run physical experiments to validate and evaluate the proposed model by comparing simulated and observed results;

iii. Identify contributions and applications of the proposed modeling approach as they relate to the larger picture of predicting realistic burr growth in drilling.

In the pursuit of achieving these objectives, an existing burr growth model was first assessed and adopted. The first model implemented was developed by Kim and Dornfeld [12] for burr formation in ductile materials. They describe the burr in terms of height and thickness and define the burr formation mechanism by the drilling thrust force and the concurrent material deformation. Factors considered in this model were drill bit geometry, drilling parameters, and material characteristics, all under ideal 
drilling conditions. These factors will all vary in a machine shop or on a production floor. The initial research done as part of this study was to utilize the model in [12] to develop a sensitivity matrix to examine the effects of the variation of key drilling process elements of burr formation. The goal was to arrive at a set of parameters through optimization that would deliver the smallest burr height and thickness together with the lowest rate of change of burr height and thickness. The burr sensitivity model, however, could not completely account for the tool and process variabilities that contribute to burr formation. Feed and point angle demonstrated opposing trends with respect to the rate of change of burr height and thickness growth. Both burr height and thickness and the rates of change of burr height and thickness increased with respect to an increasing point angle. For feed, burr height and thickness increased as feed increased, but their rates of change decreased with an increasing feed. Although a gradually increasing point angle does not automatically indicate tool wear, minimal changes in the point angle, considered as a continuous variable at that stage of research, could signal changes in the tool shape, arising from drill bit cutting edges being progressively worn down. This led to the conclusion that drill bit wear should be investigated further. When a drill bit experiences wear, the worn cutting edge radius increases and becomes rounded, consequently changing the initial shape of the cutting edge, and the drill bit transitions from cutting to ploughing.

Consequently, a second method, that of Waldorf, DeVor, and Kapoor, [15], was adopted. They established a ploughing force model, based on a slip-line field for a sliding rigid wedge merged with orthogonal cutting with a worn cutting edge. A simplified version is shown in Fig. 1.4 [10].

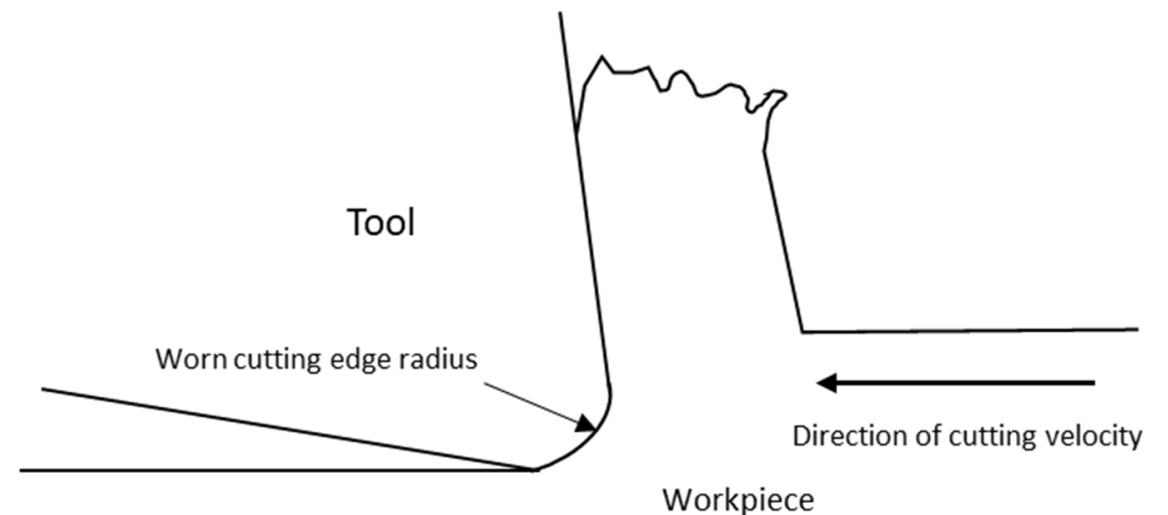

Fig. 1.4 Orthogonal cutting with a worn tool, adapted from [10, Fig. 2.4.1]

The result of combining the abovementioned models was a new thrust force for burr height and thickness growth. It utilized a segmented cutting edge whereby the thrust force acts on each segment individually and applies a weighted model that takes into account varied wear along a drill bit cutting 
edge. The novel model was evaluated in two ways. First, through a simulation and second, through experimentation. For both the simulation and experiment, five drill bits at various levels of wear, two aluminum alloys, and three different feeds were used. For the simulation, the material, tool, and process parameters were calculated to generate burr heights and thicknesses. In the experiment, the drill bits were used to drill aluminum sheet metal at the varied feeds. The resulting burr heights and thicknesses were measured, analyzed, and compared to the simulation outcomes.

\subsection{Thesis Organization}

In Chapter 2, literature on burr formation and properties, deburring processes, parameters' influence on burr formation, and tool wear are presented.

Chapter 3 covers the drilling burr formation model for sharp tools and sensitivity analysis of burr formation.

Chapter 4 is an analysis of the ploughing force in orthogonal cutting and includes the development of the proposed concept of combining drilling burr formation with tool wear, and a mathematical simulation of the model. Simulation results are also analyzed.

Chapter 5 covers the experimental validation of the new methodology.

The conclusion and recommended future work are described in Chapter 6. 


\section{CHAPTER 2 BACKGROUND AND LITERATURE REVIEW}

\subsection{Burr Formation}

Burr formation has been investigated extensively since the late 1950s. At that time, US and German researchers had a physical comprehension of how burrs developed in punching operations. This led to a development of burr size standards and of measuring tools throughout the 1950s and 1960s, to enable press-die designers and operators on the floor to change the dies before burrs grew too large and contaminated other machines [7], [16].

Machining burrs and their origins were first researched and discussed by Gillespie in his master's thesis [17] and in numerous subsequent publications. In his 1975 report "The Burrs Produced by Drilling," Gillespie discussed factors affecting burrs resulting from drilling stainless steel [6]. Other researchers focused on features of drill geometry: diameter, point angle, and length to diameter hole ratio [13], [18]. The influence of drilling process parameters on burr height and thickness, including feed and cutting speed, has also been examined in [8], [11], [19] - [21] and is further discussed in Section 2.4.

The burr formation mechanism differs depending on material ductility, drill geometry, and process conditions, and especially on feed. The size and shape of the drilling burr varies depending on the process parameters of the operation. The effects of the remaining circumstances for burr formation in drilling are described in the following.

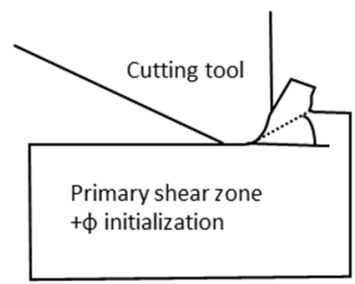

(a) Stage I

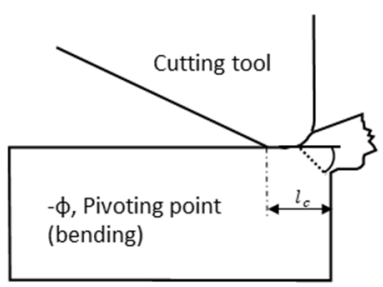

(c) Stage III

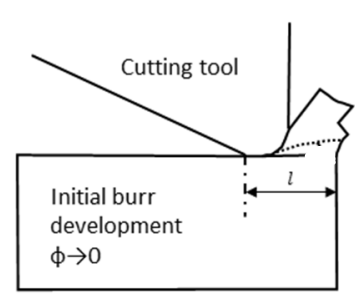

(b) Stage II

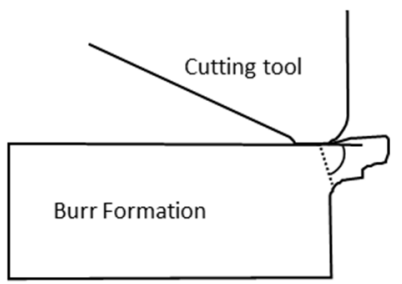

(d) Stage IV

Fig. 2.1 Four stages of burr formation, adapted from [22, Fig. 30] 
Burr formation in machining can be divided into four stages: initialization, initial development, bending, and formation of burr [22]. These are shown in Fig. 2.1. In stage I, a chip is generated by the tool, with a positive shear angle $+\phi$. As the cutting tool moves forward to the edge of the workpiece, the plastic and primary shear zones are lengthened in the direction of the edge of the workpiece. A key position in chip development is reached, beyond which the shear angle only decreases, negative shear deformation is initiated, and the initial development of the burr takes place (stage II). A burr formed from negative shear deformation is a tear burr related to cutting edge chipping in milling tools. It is similar to a rollover burr, which has been widely studied, and therefore appropriate to use to portray burr formation. In Fig. 2.1, $l$ and $l_{c}$ represent the distance between the chip development point and the edge of the workpiece. In stage III, the shear angle continues to get smaller, eventually reaching zero, negative shear is maintained, and a pivoting point is reached by the cutting tool on the workpiece. It is at this pivoting point that the workpiece edge begins to pivot together with the chip. In stage IV, the chip remains attached to the workpiece edge, and a burr is formed. The four stages of the mechanism of burr formation are then completed [22].

Dornfeld, Kim, Dechow, Hewson, and Chen [23] examined the mechanism of burr formation and developed burr formation models for drilling based on orthogonal cutting theory. The resultant burr height and thickness models demonstrated that while cutting conditions had a limited effect on burr size, drill geometry had a more pronounced effect on burr height and thickness. Kim and Dornfeld [12] furthered these studies and established a model predicting burr height and thickness in ductile materials for low and medium stainless steel. It was based on the principle of energy conservation and metal cutting theory, and considered material and tool properties and process conditions. The proposed concept in this dissertation draws on their theory.

Sofronas [24] proposed an analytical model for burr formation to determine drilling parameters and conditions that would minimize burr height and thickness. He found that by reducing either the point angle or feed and increasing the drill's helix angle, the size of the burr was reduced. Matsumura, Leopold, and Heidelberg [25] presented an analytical model focused on burr formation in drilling to predict the cutting force based on minimum cutting energy. Their model considered the cutting force and chip flow direction as relevant parameters in predicting burr formation. The force model presented by these scholars was found to be effective when utilized in the design of drill geometry where burr minimization was intended. 
In this dissertation, the focus placed is two-fold: 1) key drilling parameters that influence burr formation and 2) burr formation with tool wear, while considering the key drilling parameters.

\subsection{Burr Properties}

\subsubsection{Machining Burrs}

Gillespie and Blotter [6], [7] identified four basic types of burrs: the Poisson burr, the rollover burr, the tear burr, and the cut-off burr. These burr types are generated by specific processes and machining operations. Gillespie estimated that there were over 5,000 machining operations in existence, with most of the burr-related literature focusing on drilling, milling, and press-working processes. In the following is a summarized classification of the four most common types of burrs and their commonness in machining processes.

1) The rollover burr is a chip pushed out of the cutter's path; it is not sheared. This burr occurs when the chip bends rather than being cut or fractured. There is usually a long burr at the end of a cut, which is the rollover burr. A rollover burr occurs when a tool passes over a groove or the cut is interrupted. A smaller rollover burr forms in the case of a facing cut.

2) The tear burr forms when chips are torn from a workpiece, comparable to a burr emerging from press working operations. These burrs can form in most cutting processes. They are common in side-milling operations and drilling operations. Groove-producing processes generate tear burrs.

3) The Poisson burr results from the tendency of a material to bulge in compression. The extent of this deformation is a function of workpiece material, the size and shape of contracting components, and the applied load. The resulting burr is relatively small and is proportional to the radius of the cutting edge. Rubbing between a grooving tool and the groove walls may induce a Poisson burr by inducing material to flow out the sides of the groove. Turning operations often create Poisson burrs.

4) The cut-off burr is formed when the workpiece separates from bar stock, prior to the separation cut being made. The workpiece is usually allowed to fall from the stock before the parting toll severs the material. This is material that has not yet been cut off. It is usually found on saw cuts and components of automatic screw machines. At times this burr can be prevented by supporting the workpiece until the cut-off is complete. 


\subsubsection{Drilling Burrs}

In drilling, the burr formation mechanism differs depending on material ductility, drill geometry, and process conditions. The size and shape of the drilling burr varies depending on the process parameters of the operation. In most materials, at a low feed and cutting speed the drilling burr will end up with a uniform shape along the hole circumference. Table 2.1 summarizes the conditions under which drilling burrs form. The photographs of the burr heights of the various burr types were taken by the author during experiments described in Chapter 5.

Table 2.1 Three common drilling burrs and associated drilling burr formation mechanisms

\begin{tabular}{|c|c|c|}
\hline \multicolumn{2}{|r|}{ Uniform Height Burrs } & Description \\
\hline & With drill cap & $\begin{array}{l}\text { Moderately ductile material; as hole drilling is being completed, a drill cap } \\
\text { is pushed out ahead of the drill, which results in a small and uniform burr } \\
\text { height and thickness around the hole circumference [12], [26], [27]. }\end{array}$ \\
\hline & cap & $\begin{array}{l}\text { If the material is not moderately ductile, plastic deformation is limited } \\
\text { and the material will fracture early in the centre region of the drill. } \\
\text { Continuous cutting takes place up to the final stage of drilling, resulting in } \\
\text { a uniform burr without a drill cap [12], [26], [27]. }\end{array}$ \\
\hline Nor & -Uniform Height Burrs & Description \\
\hline & Crown burr & $\begin{array}{l}\text { The crown burr has a large and non-uniform burr height; it occurs when } \\
\text { the outer cutting edge of the drill is significantly worn down; material } \\
\text { beneath the drill is pushed ahead instead of being cut, and there is a } \\
\text { higher probability of initial fracture occurring in the centre of the drilling } \\
\text { region, creating a crown burr [12], [26], [27]. }\end{array}$ \\
\hline
\end{tabular}

\subsection{Deburring Processes}

At this point, it is worthwhile to take a closer look at the set of deburring processes that research in this dissertation is focused on reducing. In the majority of drilling operations, burrs will form as the drill enters and exits the part. In a study of German automotive and machine tool industries, Leitz, Aurich, and Franke [28] focused on costs associated with burr minimization, deburring, and part cleaning. Their findings revealed that burrs cause an increase of about 15\% in manpower and cycle-time expense, a $2 \%$ share in the rejection rate and a $4 \%$ share in machine breakdown times. Cumulatively, this meant that deburring accounted for up to $9 \%$ of the total manufacturing cost. 
There are multiple ways to deburr a drilled hole. However, each method requires resources that are better utilized in other aspects of part completion or assembly, where a burr is not created to begin with. The following summarizes several common deburring methods. This explains the level of resources required to remove burrs, which becomes especially laborious with large volumes of features to be deburred.

Most machining processes are followed by cost-intensive cleaning and deburring processes. There are several widely applied deburring methodologies: mass finishing, hard deburring, automated finishing performed with CNC machine robots, and electrochemical deburring. Mass finishing, typically used for small parts, involves mixing and shaking parts in bulk with abrasive media in a container. This method deburrs entire surfaces. Hard deburring methods are cutting processes, most often manual, using knives, scrapers, and rotary burrs. This method is particularly used in robotic deburring [7], [29].

Automated/robotic deburring is achieved with CNC machining centres that manufacture entire parts and can be used effectively to deburr as well. Robotic deburring and finishing systems are commonly used for high quantities of parts, including mass production volumes needed for the automotive industry. Automation resolves many issues associated with manual deburring, such as its labor intensity and risk of musco-skeletal injury for the workers, but it is not without challenges, as it necessitates indepth knowledge of deburring processes and does not guarantee ideal results. Automated deburring occurs in one of two ways: either deburring tools utilized by robots do the deburring or CNC machines are used [30]. Tube metal brushes are well suited and commonly used in automated deburring. They are available in many types of materials, stiffnesses, and diameters [4], [7].

Electrochemical deburring (ECD) consists of pumping an electrolyte solution through a space between a tool and a metal part, where the tool is the cathode and the metal part is the anode, completing the electric circuit. The current running through the circuit dissolves the burr via an electrochemical reaction. The tool and the workpiece never come into contact. Therefore, no additional mechanical or thermal stresses are exerted on the part, and the physical and chemical properties of the part remain unaltered. Its dimensions remain unchanged [31].

As can be seen from the above sources, though a variety of methods of removing burrs exist, even based on a limited high-level list, each method requires additional time and resources that, should the burrs not have been produced in the first place, could be dedicated to other aspects of manufacturing or machining. 


\subsection{Influence of Parameters on Burr Formation}

Drill geometry, workpiece material, and size are all important factors affecting burr size; exit-burr thickness has been observed to be directly related to the exit burr height. Gillespie's "Formation and Properties of Machining Burrs" [6] addressed the characteristics of all machining burrs. About 10 years later, Pande and Relekar [13] examined the influence of machining process parameters on burr thickness and height-drill diameter, feed, length to diameter hole ratio, and work material hardnessby applying machining and cutting theories and through a response surface methodology technique relating process responses to process parameters. Using experiments, the authors established an optimal feed range for minimal burr growth. They concluded that feed was unconnected to other drilling and material parameters. They further inferred that through continuous feed modification at the entry and exit of holes, significant burr size reduction was achievable for a range of materials, including steel and aluminum.

Saunders and Mauch [18] and Lauderbaugh [11] conducted several studies focusing on drilling parameters and resulting burr growth. They developed an exit burr model for drilling metals, enabling the prediction of a burr height based on operating parameters and ranges. Orthogonal cutting burr formation behaviours from past studies were extended to include indentation, and orthogonal and oblique cutting to create a burr prediction model. Temperature and thrust force were determined to influence burr size together with feed rates. Their simulation and experiments led to several conclusions: Burr formation depended significantly on material yield strength and ultimate strength, high feed rates during drilling and low feed rates at exit minimized the burr size, and the dominant parameters were the point angle and feed rates, together with a reduced cutting edge of the drill bit.

Huang and Tosun's [19], [20] published works utilizing machining theory, grey-relational analysis, and the Taguchi method focusing on drilling parameters and burr height. Huang and Tosun developed optimal drilling parameters for multiple performance characteristics: tool life, surface roughness, and burr height. When looking to optimize drilling Al 6061, they used the grey-Taguchi method to achieve optimal surface roughness. The key parameters they were looking to optimize were coated deposition, spindle speed, and feed rate. They proved that these factors, when combined in the proportions optimised with the Taguchi method, delivered the improved performance characteristics they sought. It is important to note that among the many parameters they could have selected, feed rate was in the top three they chose to examine, underlining its importance. 
Tosun's study [20] established recommended levels of drilling parameters to achieve the desired surface roughness and burr height. He found that the drill itself had the strongest influence of all drilling parameters. In the order of decreasing influence, the factors were drill type, cutting speed, feed rate, and point angle. Although workpiece material and coolant conditions were also examined as they typically have a strong impact on machining efficiency, cost, quality of the machined part, feed, and point angle emerged in the set of drilling parameters most influencing burr height and surface roughness.

Brinksmeier and Fangmann [21] examined the effects of orbital drilling on cap and burr formation in aluminum to study the influence of different tool geometries, coatings, cutting parameters, tool wear, and lubrication on the cap and burr formation. Orbital drilling is used extensively in the aerospace industry for cutting composite materials and rivet machines (e.g., flap tracks and vertical tail planes). Therefore, although Brinksmeier and Fangmann examined orbital drilling and this dissertation focuses on standard drilling, their study remains relevant to this body of research. It described the best tool and machining parameters for orbital drilling to achieve the best cap formation, including cutting force, cutting, orbital speeds, and drilling feed. Brinksmeier and Fangmann found high axial feed rates to be the best for removing a drilling cap, while low feed rates produced the most accurate holes. Therefore, they identified feed as a parameter of key importance in drilling cap formation.

Using cutting theory, Basavarajappa, Chandramohan, Ashwin, Prabu, and Mukund [8] studied the influence of cutting parameters on burr formation in drilling metal matrix composites (MMCs) with carbide and coated carbide drills. They established a correlation between variables and burr formation through linear regression equations, enabling the prediction of burr height formation in drilling MMCs with a reasonable degree of accuracy. The feed rate was found to have a greater statistical and physical consequence on the resulting burr height, more so than cutting speed, further solidifying the relevance of this parameter to burr growth.

Employing machining theory, Gaitonde, Karnik, Achyutha, Siddeswarappa, and Davim [14] investigated the influence of process parameters: cutting speed, feed, drill diameter, point angle, burr height on exit, and thickness from drilling AISI 316L steel. They modeled the drilling process using response surface methodology, which showed that the methodology accurately predicted process responses and indicated feed, drill diameter, and point angle to considerably influence burr formation. 
Abdelhafeez, Soo, Aspinwall, Dowson, and Arnold [32] studied the effects of two drilling parameters, cutting speed and feed rate, on burr growth and hole quality by examining the results of drilling experiments on titanium and aluminum alloys. The researchers targeted the ability of burr minimization to decrease the need for deburring operations. The feed rate and the interaction between cutting speed and feed rate were found to have a considerable implication with respect to exit burr size for drilling workpiece materials.

\subsection{Impact of Tool Wear on Burr Formation}

When reviewing the factors that contribute to burr formation, calculations with basic drilling parameters assume ideal drilling conditions, utilizing perfectly sharp drill bits with infinitely small edge radii. The tool wear mechanism acts steadily and gradually, with the tool material progressively being removed where the workpiece and tool come into contact [1]. Tools experience wear and tear at different rates, depending on tool and workpiece material.

When a tool can no longer satisfactorily manufacture a part (e.g., dimensions and surface finish are out of tolerance, not in accordance with the drawing) it is taken out of use [29]. Accordingly, whether a drill bit can remain in service depends on if it can still produce the desired hole within the required drawing tolerance, and within an economically feasible time.

In cutting, tool wear progresses over time at two main locations, the flank and the rake face [31]. Crater wear and flank wear are two wear processes acting at these locations. Crater wear typically introduces wear to the top rake face of the tool. It is generated through the action of the chip sliding against the tool surface, resulting in the formation of a concave, crater-like section.

Flank wear is the result of friction between the newly machined workpiece surface and the flank face of the tool (also referred to as the relief face), which is adjacent to the cutting edge. This action produces a wear land. Flank wear occurs in every machining operation [29]. When the flank and the machined workpiece come into contact during tool use, tool particles occasionally attach themselves to the workpiece face and are sheared off, impairing the tool. Heat and, consequently, friction, together with flank forces, are also generated as part of flank wear, jointly contributing to deviations throughout the cutting process, culminating in unwanted variances in final part dimensions. Once a chip is formed, secondary shear is generated in the secondary shear zone, resulting from the friction between the tool and the chip. Where the tool flank scrapes against the freshly cut work surface is known as the tertiary 
shear zone, which can lead to flank wear (for more details regarding secondary and tertiary shear zones see Appendix A, sections A.1 and A.4.1). The work in this dissertation focuses on the primary shear zone. Therefore, flank wear will be reserved for examination in future work. Wang, Huang, and Song [33] focused on the effects of tool flank wear in orthogonal cutting, which showed that an additional ploughing force on the tool wear land led to a considerable surge in individual force components. The study also demonstrated that the thrust force component exhibited greater responsiveness to tool flank wear. Abdelhafeez, Soo, Aspinwall, Dowson, and Arnold [32] also studied the effects of cutting speeds and feed rates on tool flank wear. They found that drilling with drill bits with increased cutting edge radii led to ploughing, and consequently increased the feed and drilling thrust forces, yielding greater burr heights at hole exits. These studies could serve as the basis for future drill bit tool wear examination and monitoring approaches.

Flank wear and crater wear geometries on a single point tool can be found in [34 (see Fig. 2.28, p. 54)]. Photographs of both can be found in [31 (see Fig. 23.2, p. 536)].

Wear mechanisms that commonly introduce wear in interfaces between both the tool and the work, and the tool and the chip during machining, as described in [29], [31], [34] are 1) abrasion, where small fractions of the tool are scooped out by hard particles from the work material, commonly leading to flank wear; 2) adhesion, where welding occurs between the tool and the work due to the high pressure and temperature between the two, leading to small pieces of the tool breaking away with chip flow; 3 ) plastic deformation of the cutting edge, where cutting forces at high temperature advance flank wear and plastically deform the cutting edge; 4) diffusion, a key contributor to crater wear, consisting of an exchange of atoms between the tool and the wear at the tool-chip interface, making the tool more vulnerable to adhesion and abrasion; and 5) chemical reactions, where the rake face of the tool is oxidized as a result of high-speed machining and accompanying high temperatures where the tool and chip come into contact; the oxidized coating is sheared away, baring the raw tool surface to continue with cutting operations. Thermal softening is an additional wear mechanism related to adhesion and abrasion wear. Rates of adhesion and abrasion are greater when the cutting speed is increased because greater cutting speed elevates cutting temperatures. In turn, elevated cutting temperatures lead to a decrease in the hardness of the tool material. This is termed thermal softening. Thermal softening could also lead to plastic deformation of the tool cutting edge.

Fig. 1.2 illustrates twist-drill geometry and its detailed features. A subset of the aforementioned set of cutting tool wear mechanisms is applicable to the specific ways that twist drill bit geometry features 
typically experience wear [29]. These features are chisel edge wear, margin wear, crater wear, and cutting edge wear (also referred to as lip wear). A diagram of these can be found in [29 (see Fig. 9.24)]. The wear mechanism relevant to this dissertation is cutting edge wear.

Chisel edge wear can lead to a doubling and tripling of the drilling thrust force and impact the centering accuracy of the drill bit. Chisel edge wear stems from plastic deformation or abrasion [29].

Margin, or land wear, develops on the land that touches the drilled surface or the outer corner of the cutting edge. Diffusion, abrasion, and thermal softening can all induce margin wear. Margin wear in drill bits delivers undersized holes and poor surface finishes.

Crater wear ensues diffusion or thermal softening and is found on the flute surface. Whereas substantial crater wear can initiate lip breakage, chipping, or deformation as it weakens the cutting edge, in moderate amounts it is not problematic.

Lip wear is a consequence of plastic deformation, abrasion, or incorrect point grinding, which leads to a lack of adequate flank relief, allowing the drill bit to linger in the base of blind holes [29]. This dissertation examines aspects of drill bit cutting edge wear and its influence on burr height and thickness growth. This is significant because cutting edge wear causes greater burr growth through holes and increases in the drilling thrust force. Cutting edge wear also increases maximum cutting edge temperature and power consumption. However, these are not the focus of this research. Additional motivations for this research will be provided in the following paragraphs.

The particular mode of wear most relevant to drill bit wear and burr formation is drill bit cutting edge wear, as it increases both the burr growth and the drilling thrust force. Moreover, as mentioned above and as elaborated on in Appendix A, Section A.4, the work in this dissertation focuses on the primary shear zone. In orthogonal cutting, a chip is formed when the cutting edge of the tool makes contact with the workpiece and is sheared; this occurs in the primary shear zone. Continuous tool use will eventually lead to cutting edge wear. This is directly linked to drill bit cutting edge wear, as the drilling mechanism is rooted in orthogonal cutting. Furthermore, drill bit cutting edge wear and rounding is examined in greater detail by Faraz, Biermann, and Klaus [35] and Luo, Zhang, Li, Cheng, and Liu [36], who studied drilling of composite materials. Cutting edge wear for metals is not well examined, likely because of the lightweight properties of composites and their ability to deliver structural strength for aerospace applications [36]. Considering these factors, drill bit cutting edge wear with respect to burr height and thickness growth in ductile materials is focus of this dissertation. 
Kim and Dornfeld [26], in their work on burr formation, articulated the relationship between a growing thrust force and a corresponding increase in burr height and thickness when drilling with sharp drill bits.

Waldorf, DeVor, and Kapoor [15] developed a model for ploughing components of a cutting force during orthogonal cutting by estimating cutting forces with a slip-line field. The researchers used large cutting edge radii to simulate cutting tool edges that were worn. They examined the relationship between thrust forces and the size of the cutting tool radii during turning experiments. The model adequately predicted the ploughing forces present when performing cutting operations with the large edge radii, delivering a better understanding of the relationship between cutting edge radii on cutting forces. The ploughing force model developed in [15] links an increased cutting edge radius to a correspondingly incremental ploughing thrust force.

\subsection{Slip-Line Field Models}

Dewhurst and Collins [37] developed a technique for solving problems involving plane strain rigid perfectly for plastic models. Theirs is the fundamental slip-line theory referenced by the majority of slipline researchers. They established procedures that enabled the construction of an indirect slip-line solution that culminated in linear integral equations and a script for a computer program. At the core of this method is the solution to a subset of mixed boundary value problems. Although this approach is commonly used for orthogonal cutting models, the authors utilized strip rolling to illustrate the process. With respect to force calculations, which are at the core of to the models described below, in this study the geometry of the slip-line field was calculated first, followed by the evaluation of the total force and moment transmitted across slip lines to establish the drawing force, or roll torque, as applicable to Dewhurst and Collins' work. In other scenarios it could be the thrust force or the ploughing force. This same technique was followed by subsequent scholars applying Dewhurst and Collins' process, including Waldorf, DeVor, and Kapoor [15] for their ploughing force model. The methodology developed in this dissertation, utilizing slip-line field theory, is also rooted in Dewhurst and Collins' model.

The model mentioned in Section 2.5, developed by Waldorf, DeVor, and Kapoor [15] for calculating worn tool cutting forces for orthogonal cutting based on a linear accumulation of the plastic flow regions, is based on slip-line fields for a sliding rigid wedge. Their model addressed the reality of uncut chip thickness being directly related to the cutting edge radius and a ploughing element of the forces becoming significant as compared to the acting shear forces. Waldorf, DeVor, and Kapoor's model 
approximated the total forces experienced in orthogonal cutting and demonstrated the relationship between the larger, more worn tool cutting edge radius and the resulting ploughing force. The formulations in this dissertation draw on their work. As their model is based on a slip-line field model, it deserves more discussion here.

The machining simulation field has utilized slip-line models since 1945. Initial slip-line models are described by Leopold [5], [38 (see p. 74, Fig. 4)]. Fig. 2.2 shows a more recent wave model for wedge sliding [15], [39].

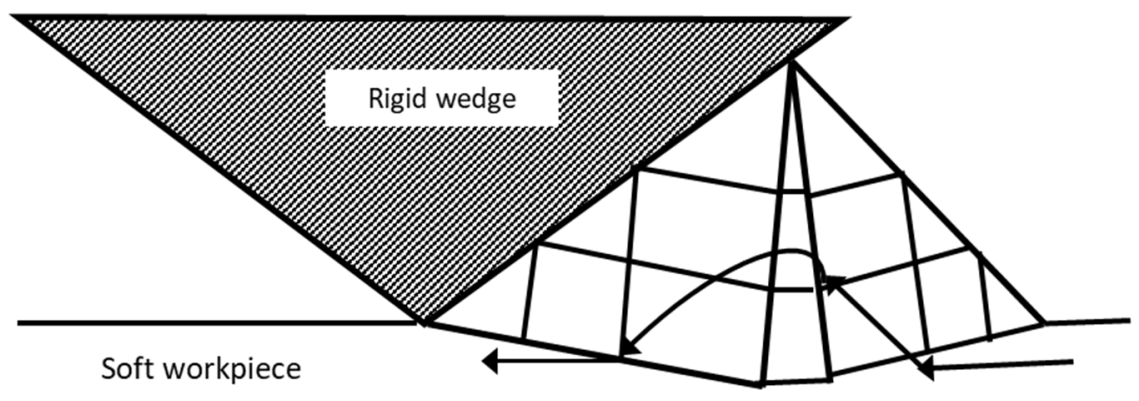

Fig. 2.2 Slip-line field for wedge sliding, adapted from [15, Fig. 2], [39]

The workpiece material is pushed in a wave-like motion ahead of the rigid wedge and is then compressed and forced upwards to come into contact with the face of the wedge (see Fig. 2.2). The arrows indicate the direction of material flow.

Shi and Ramalingam [40] developed an earlier slip-line field for orthogonal cutting with a chip breaker and flank wear. Their slip-line field accounts for tool wear via a primary deformation zone with finite thickness, two secondary shear zones, a curled chip, and a flank force system. This method successfully presented a linear relationship between tool flank wear and components of the cutting force. Similar to the drilling and tool wear findings discussed above, where drilling thrust forces increased with increased drill bit wear, Shi and Ramalingam demonstrated that with increased tool wear, a growth occurred in cutting force components. They recommended using the variance in cutting forces to calculate a cutting force component ratio that could serve as a practical method of monitoring tools for flank wear in automated machining.

Chinchanikar and Choudhury [41] established a cutting force model under oblique cutting conditions considering tool wear, tool life, geometric tolerance of the finished product, and surface finish. Their approach was validated with the experimental results from turning hardened AISI 4340. Cutting forces 
resulting only from flank wear effects were modeled by extrapolating Waldorf's orthogonal force model to 3D cutting force analysis. Experimental results demonstrated that the cutting forces from flank wear were impacted mainly by the amount of wear and, to a degree, rely on the cutting condition that generated the tool wear. Burr formation was not studied by the researchers as it was not applicable for their model. Therefore only tool wear and its impact on cutting forces was addressed.

Long, Huang, and Sun [42], [43] developed a slip-line field force model to forecast cutting forces in orthogonal cutting when using a tool impacted by both flank and crater wear. Their study resulted in several slip-line field models: for the primary shear zone, flank wear land, and each part of the secondary shear zone. The slip-line field models, in turn, enabled the development of a worn force model that successfully portrayed the joint contribution of the total cutting forces together with the force variations expected as tool wear progressed.

\subsection{Drilling Burr Formation with Tool Wear}

While cutting and thrust force models, including tool wear, have been thoroughly investigated for orthogonal cutting, there appears to be a research gap in establishing a drilling thrust force accounting for both drilling parameter and tool wear focusing on burr growth (i.e., increasing height and thickness) that allows the prediction of burr size depending on the percentage of tool wear.

Contemporary trends and recommendations with respect to studying burr growth are moving towards predictive modeling and burr minimization. Leopold and Wohlgemuth [5] examined the methods currently utilized in burr formation modeling and simulations. They concluded that theoretical modeling of burr formation is the basis of future research efforts for burr avoidance and minimization, as opposed to research revolving around material removal as burrs are formed. The modern and practical path forward should be focused on predictive modeling with an aim at burr minimization. Numerical models will lead to a better understanding of burr formation and minimization. Future analytical models should focus on real three-dimensional objects, new material properties, and new algorithms and computation principles to increase modeling efficiency. In the past, numerical investigations were an arduous process, but going forward, better methods for predictive modeling, including virtual machining, should be considered instead. The approach for predictive modeling is the path being pursue in this dissertation. By enhancing an existing burr growth model and its adaptation to reflect real-life drilling conditions, allow for the forecasting of a burr with partial wear drill bit conditions present. 
A drilling burr height and thickness model accounting for the impact of tool wear on burr growth does not exist. Dornfeld and Kim's [12] model assumes ideal drilling conditions, utilizing a sharp drill bit. Waldorf, DeVor, and Kapoor's [15] model for ploughing forces in orthogonal cutting provides a practical approximation of the impact of a cutting edge with a large radius. This has been adapted to drilling in this dissertation.

This dissertation will focus on addressing the research gap for burr height and thickness in drilling. The proposed concept introduces a methodology for a wear/no-wear condition via percentage wear for a drill bit cutting edge, drawing on Dornfeld and Kim's [12] model for sharp drilling burrs and Waldorf, DeVor, and Kapoor's [15] model for ploughing forces. 


\section{CHAPTER 3 DRILLING BURR FORMATION AND BURR SENSITIVITY ANALYSIS}

This chapter begins with a presentation of an existing burr formation model, followed by the development of a burr sensitivity matrix to facilitate the analysis of the impact of key drilling process parameters on burr formation.

\subsection{Drilling Burr-Formation Model}

The burr formation model for drilling to model burr height and thickness presented here is based on the work of Kim and Dornfeld [12]. This model was founded on the conservation of energy and work during burr formation, starting with the basic work and energy equation, where work done equals energy transferred. In order to drill through ductile materials, a combination of thrust force and material deformation will produce a hole and, inevitably, a burr, a by-product of drilling. Their model assumes that the total work required to drill through and form a burr is the sum of the work of the thrust force applied by the drill and of the work of material deformation.

The burr formation mechanism under scrutiny is illustrated as a four-phase drilling process.

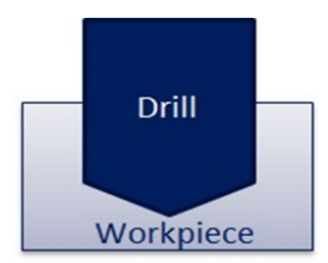

Phase I

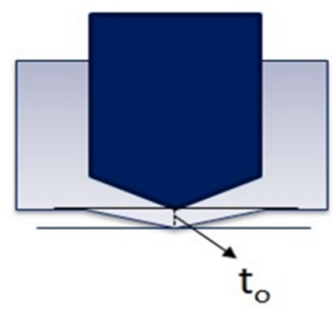

Phase II

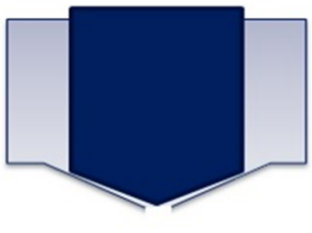

Phase III

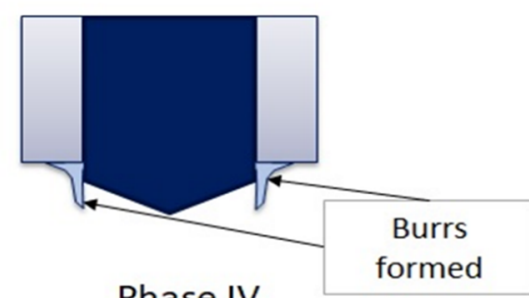

Phase IV

Fig. 3.1 Four phases of drilling, adapted from [12, Fig. 4]

As the drill bit moves forward it penetrates the work material to create a hole; this will become the exit surface. It consumes energy for cutting with no deformation occurring. Before the material fractures, it is first deformed near the centre of the drill, at which point material is no longer being cut, only deformed. As deformation progresses, before the drill exits the material, cutting occurs concurrently at 
the outer perimeter of the drill bit until the initial break in the material happens. Once the material fractures, the cutting ceases and only exit deformation takes place at that point. It is assumed that total work done, $\Delta W_{T O T A L}$, eq. (3.1) [12, eq. (1)], comprises work for material deformation, $\Delta W_{D F}$, and work of the thrust force, $\Delta W_{T H}$, during the four phases of drilling.

$$
\Delta W_{D F}+\Delta W_{T H}=\Delta W_{T O T A L}
$$

The model described in [12] was founded on the concept of total work leading to the calculation of the initial chip thickness of deforming material, $t_{0}$, which is the foundation of the culminating burr height and thickness algorithms, eq. (3.1) and eq. (3.2), [12, eq. (31)], which are critical to the research in this dissertation.

$$
\begin{gathered}
H=t_{0} \sin p \exp \left\{\frac{\sqrt{3}}{2} \ln \left(\frac{100}{100-\% R \cdot A .}\right)\right\} \\
T=t_{0} \sin p \tan p
\end{gathered}
$$

\subsection{Drilling Thrust Force}

The fundamental work equation, where work = force $\mathrm{x}$ distance, was adapted by Kim and Dornfeld for the work of the drilling thrust force, where the force is the drilling thrust force, $F_{T H}$. The work of the thrust force, $\Delta W_{T H}$, stems from the thrust force of orthogonal cutting adapted to drilling.

As detailed by Stephenson and Agapiou [2], thrust force calculation for drilling is unique because of the drill bit's overall complex geometry and low cutting speed. Forces of varying magnitudes can result from drill bits having a range of dissimilar point angles, while concurrently generating similar chip areas prior to chip formation.

The drilling mechanism was treated an oblique cutting process, stemming from orthogonal cutting, as shown in Fig. 3.2. The oblique angle varies in relation to the radial position of the drill bit cutting edge. Dividing the drill bit cutting edge into small segments as shown in Fig 3.3, enables the approximation of the total thrust force of oblique cutting [12], [29]. Applying an oblique cutting model in order to compute the forces on the drill bit cutting edge is a commonly used method in machining process analysis which can be applied to aerospace manufacturing [29]. Each segment is assumed to act as an 
individual cutting edge with a thrust force acting on it, which can be calculated. The thrust force calculation begins with utilizing orthogonal cutting to compute the force on each segment. Then a dynamic rake angle is incorporated to adapt the thrust force to drilling. This process is further described in the next section of the chapter and in Appendix A.
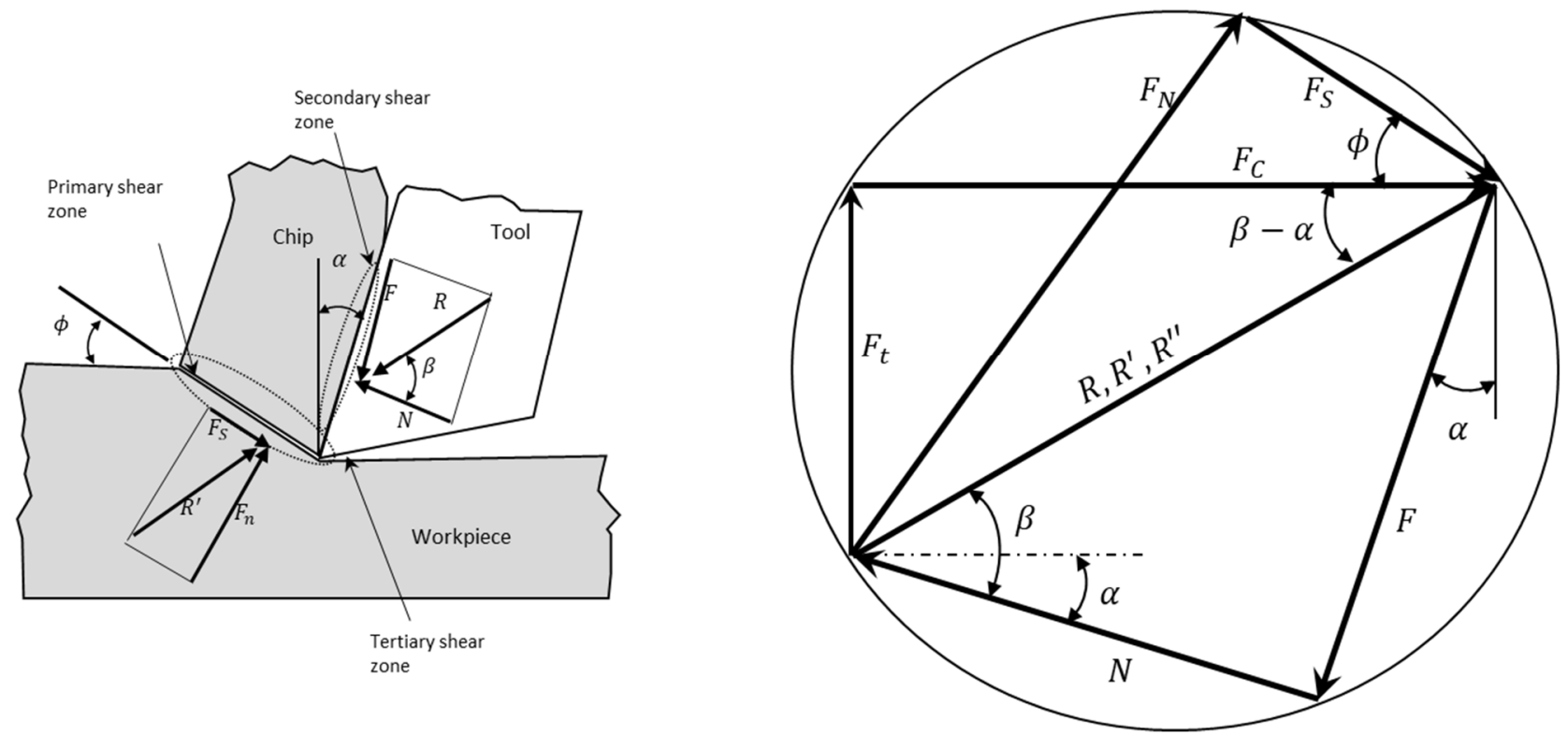

Fig. 3.2 Orthogonal-cutting model and Merchant-circle model

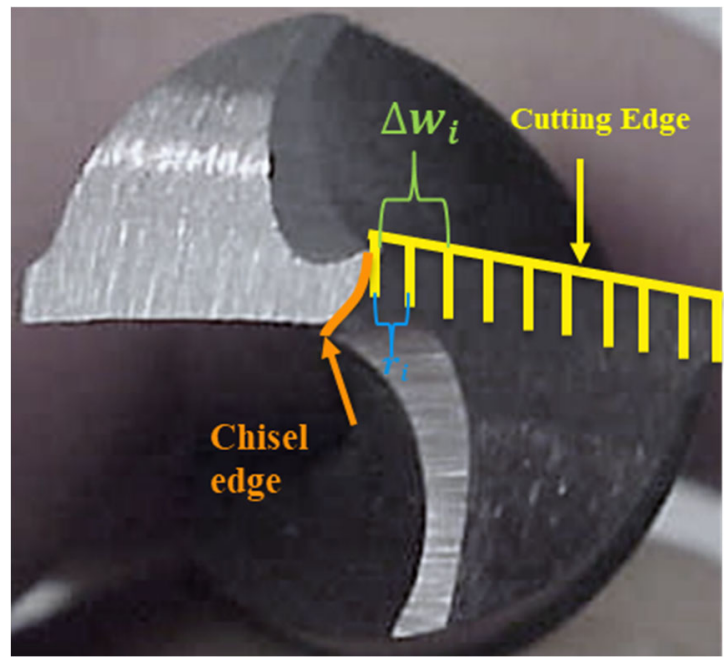

Fig. 3.3 Segmented drill bit cutting edge

In Fig. 3.3 and per the subsequent derivation, $\Delta w=\left(r_{i+1}-r_{i}\right)$ is the width of the $i^{\text {th }}$ segment, $r_{i}$ identifies a single segment, and $r_{i+1}$ is the subsequent segment following $r_{i}$. 
To determine $\Delta W_{T H}$, the fluctuation of the thrust force as the material was being drilled was examined [12]. The tests consisted of using a split-point twist drill, $3.175 \mathrm{~mm}$ in diameter, to drill stainless steel AISI $304 \mathrm{~L}$ at a feed of $0.0317 \mathrm{~mm} / \mathrm{rev}$. The thrust force during the drilling process was measured using a dynamometer.

The outcome of these observations became the work of the drilling thrust force, $\Delta \mathrm{W}_{\mathrm{TH}}$, in a quadratic equation. Subsequent derivation of the drilling thrust force, based on orthogonal cutting and the Merchant model, led to the establishment of the total drilling thrust force, $F_{T H}$. See Appendix A, Section A. 1 for the derivation and details

$F_{T H}$ incorporated into the work of the thrust force delivers eq. (3.4) for $\Delta W_{\mathrm{TH}}$, derived from [12, eqs. (3), (15)] is :

$$
\Delta W_{\mathrm{TH}}=\frac{2}{3}\left[\frac{\sqrt{2}}{3} R f \cdot \sigma_{u} \cdot \sum_{i=1}^{N}\left\{\frac{\sin \left(\frac{\pi}{6}-\frac{\alpha_{d}}{2}\right)\left(\rho_{i+1-} \rho_{i}\right)}{\sin \left(\left(\frac{\pi}{6}+\frac{\alpha_{d}}{4}\right) \cos \left(\frac{\pi}{3}-\frac{\alpha_{d}}{4}\right)\right)}\right\}\right] L
$$

where $R$ is the drill bit radius, $f$ is the feed, $\sigma_{u}$ is the ultimate tensile strength of the ductile material, $N$ is the number of segments the drill bit cutting edge is divided into, and $\alpha_{d}$ is the dynamic rake angle, a quantity based on the normal rake angle $\alpha_{n}$. The orthogonal cutting model is adapted to an oblique cutting model through the incorporation of feed, the varying radial distance from the drill bit centreline, and through $\rho$, the relative radius. $\rho$, is a ratio of the distance of each segment on the sectioned drill bit cutting edge to the drill bit radius. For the detailed derivation of $F_{T H}, \alpha_{d}, \alpha_{n}$, and $\Delta W_{\mathrm{TH}}$ see Appendix A, Section A.1.

\subsection{Material Deformation}

Work of material deformation, $\Delta W_{D F}$, is represented by eq. (3.5) [12, eq. (24)]:

$$
\Delta W_{D F}=\frac{3}{4} \pi R^{2} t_{0} \sigma_{y} \sin p\left\{\ln \left(\frac{1}{\sin p}\right)+\frac{t_{0}}{R} \sin ^{2} p \cos p\right\}+\frac{1}{2} \pi \sigma_{y}\left(\frac{\pi}{2}-p\right) R t_{0}^{2}
$$

$\Delta W_{D F}$ is obtained through modeling the drilled material as a thin plate, circular in shape, which transforms into a cone resulting from material bending and elongation. This is portrayed in Fig. 3.4. The 
detailed geometry of this transformation process is represented in Fig. 3.5. It is illustrated as a series of bending elongation steps as the shape translates from abde to $f d h g$.
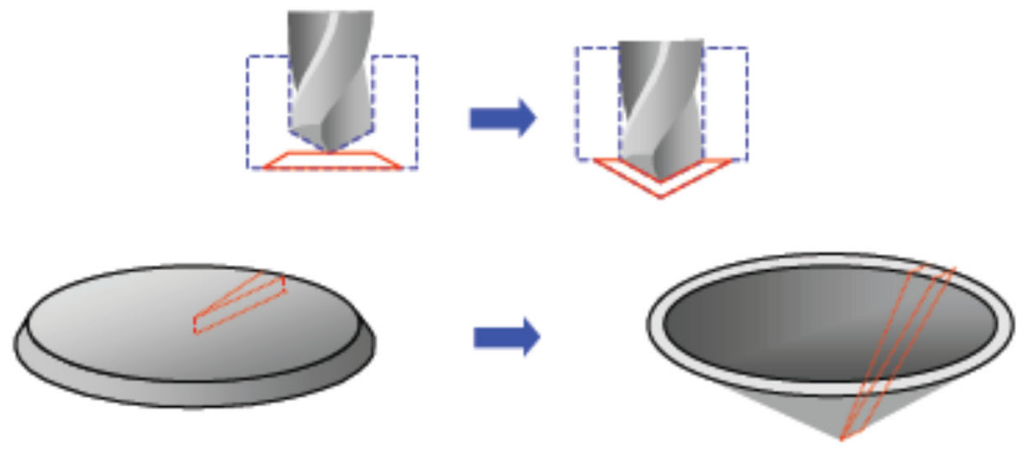

Fig. 3.4 Graphical depiction of material deformation in drilling

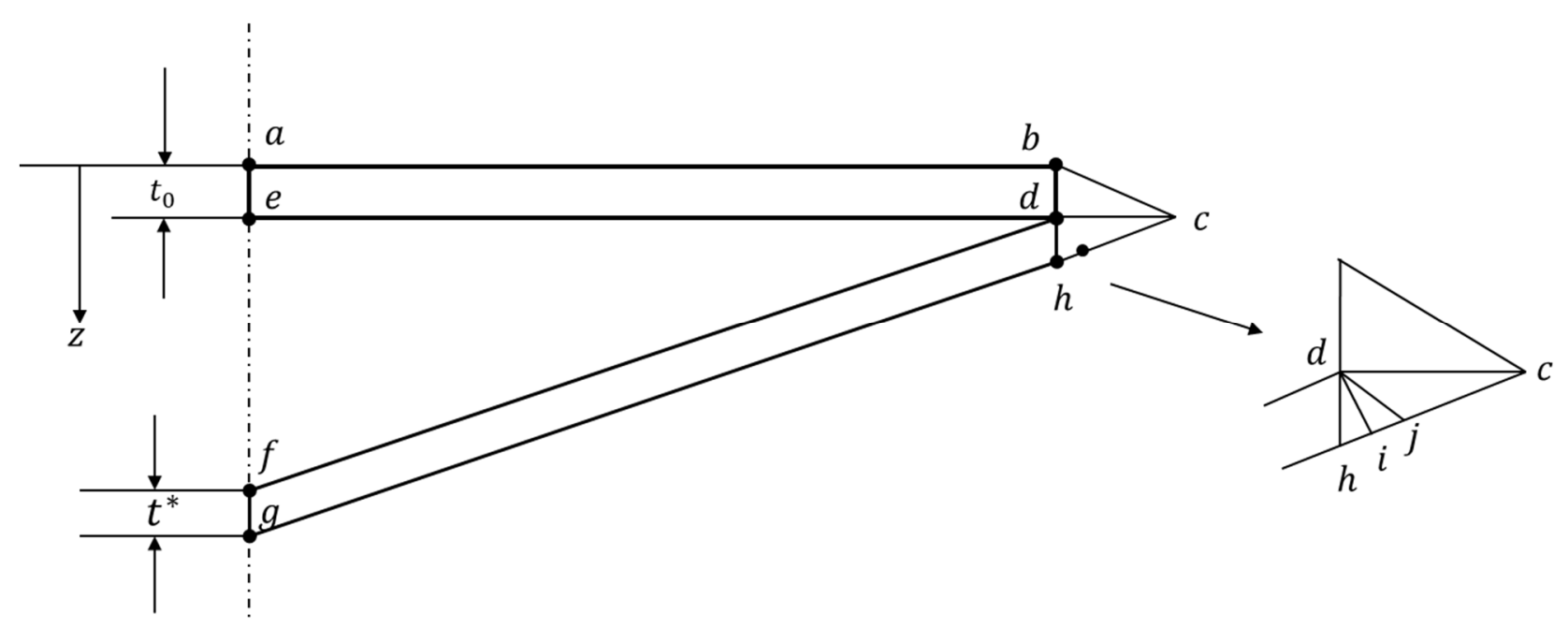

Fig. 3.5 Half cross section of material prior to and after deformation, adapted from [12, Fig. 6]

$W_{D F}$ is the sum of the work of these steps. Shear strain is also a component of the deformation, but its effects are limited to a very small region around the drill centre, and therefore the work performed by the shear strain is considered to be negligible. For the details of the derivation, see Appendix A, Section A.2.

\subsection{Final Burr Formation}

Total work is the outcome of the steady-state thrust force and the distance from the deformation initiation point (see phase 1 in Fig. 3.1) to the stage where the outer drill cutting edge is in line with the exit surface (see phase 3 in Fig. 3.1). The sum of these two work components forms a quadratic equation, the solution of which is the initial chip thickness of deforming material, $t_{0}$. The steps 
explaining how the total work equation is formed can be found in Appendix A, Section A.3. The equation representing the total work is $W_{\text {TOTAL }}$, eq. (3.6) [12, eq. (25)]:

$$
\begin{aligned}
& \frac{3}{4} \pi R^{2} t_{0} \sigma_{y} \sin p\left\{\ln \left(\frac{1}{\sin p}\right)+\frac{t_{0}}{R} \sin ^{2} p \cos p\right\}+\frac{1}{2} \pi \sigma_{y}\left(\frac{\pi}{2}-p\right) R t_{0}^{2}+\frac{2}{3} F_{t h} L=F_{t h} L \\
&=W_{\text {TотAL }}
\end{aligned}
$$

The $W_{\text {TOTAL }}$ equation is split into the following subcomponents to enable the creation of a quadratic equation, derived from [12, eq. (25)]:

Table 3.1 Subcomponents of initial chip thickness

\begin{tabular}{|c|c|}
\hline$A=\frac{3}{4} \pi R^{2} \sigma_{y} \sin p$ & $F=F_{t h}=\frac{\sqrt{2}}{3} R f \sigma_{u} \Phi$ \\
\hline$B=\ln \left(\frac{1}{\sin p}\right)$ & $L=t_{0}+\frac{R}{\tan p}$ \\
\hline$C=\frac{\sin p^{2} \cos p}{R}$ & $t \rightarrow t_{0}$ \\
\hline$D=\frac{1}{2} \pi \sigma_{y}\left(\frac{\pi}{2}-p\right) R$ & $\Phi=\sum_{i=1}^{N}\left\{\frac{\sin \left(\frac{\pi}{6}-\frac{\alpha_{d}}{2}\right)\left(\rho_{i+1-} \rho_{i}\right)}{\sin \left(\frac{\pi}{6}+\frac{\alpha_{d}}{4}\right) \cos \left(\frac{\pi}{3}-\frac{\alpha_{d}}{4}\right)}\right\}$ \\
\hline
\end{tabular}

Symbolically re-writing the $\Delta W_{\text {TоTAL }}$ equation in the form of a second-order polynomial:

$$
A t\{B+C t\}+D t^{2}+\frac{2}{3} F L=F L
$$

Assigning $J_{1}, J_{2}$, and $J_{3}$ for simplicity:

$$
\underbrace{(A C+D) t^{2}+}_{J_{1}} \underbrace{\left(A B-\frac{1}{3} F\right) t}_{J_{2}}-\frac{1}{\frac{1}{3} F \frac{R}{\tan p}}=0
$$

The solution to the quadratic equation, $t_{0}$, is as follows, as derived from [12, eq. (26)]: 


$$
t_{0}=\frac{-J_{2}+\sqrt{J_{2}{ }^{2}-4 J_{1} J_{3}}}{2\left(J_{1}\right)}
$$

The individual expanded components, as derived from [12, eq. (26)], are:

$$
\begin{gathered}
(A C+D)=J_{1}=\frac{3}{4} \pi \sin p^{3} \cos p+\frac{1}{2} \pi\left(\frac{\pi}{2}-p\right) \\
\left(A B-\frac{1}{3} F\right)=J_{2}=\frac{3}{4} \pi R \sin p\left(\ln \left(\frac{1}{\sin p}\right)\right)-\frac{\sqrt{2}}{9} f\left(\frac{\sigma_{u}}{\sigma_{y}}\right) \sigma_{y} \cdot \Phi \\
\left(\frac{1}{3} F \frac{R}{\tan p}\right)=J_{3}=\frac{\sqrt{2}}{9} f \sigma_{u}\left(\frac{R}{\tan p}\right) \cdot \Phi \\
\Phi=\sum_{i=1}^{N}\left\{\frac{\sin \left(\frac{\pi}{6}-\frac{\alpha_{d}}{2}\right)\left(\rho_{i+1-} \rho_{i}\right)}{\sin \left(\frac{\pi}{6}+\frac{\alpha_{d}}{4}\right) \cos \left(\frac{\pi}{3}-\frac{\alpha_{d}}{4}\right)}\right\}
\end{gathered}
$$

Note that eqs. (3.17) and (3.18), represented by $X$ and $Y$ in [12, eq. (26)], differ. When completing the rederivation of the model, irregularities appeared between the rederivation results and the content in [12]. After completing a detailed review of the rederivation, it was concluded eqs. (3.11) and (3.12) were correctly rederived.

$t_{0}$, together with the tensile property of reduction of the area at fracture, the geometric relationship with respect to material deformation, the examination of the volume-conservation principle of plastic deformation, and material fracture strain, enables the final formulation of the thickness of deformed material, $t_{0} \sin p$, burr height, $H$, and thickness, $T$, [12, eq. (31)]. The research in this dissertation focuses primarily on $H$ and $T$.

$$
\begin{gathered}
H=t_{0} \sin p \exp \left\{\frac{\sqrt{3}}{2} \ln \left(\frac{100}{100-\% R \cdot A .}\right)\right\} \\
T=t_{0} \sin p \tan p
\end{gathered}
$$




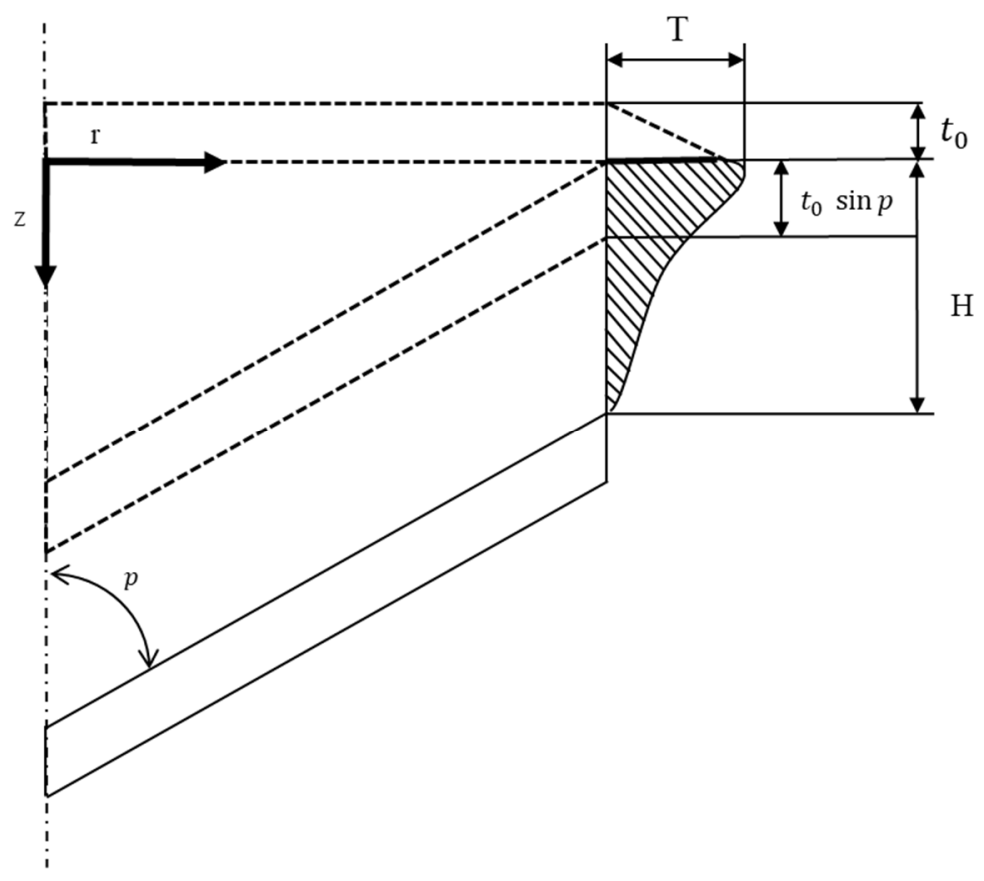

Fig. 3.6 Burr height and thickness formation, adapted from [12, Fig. 7]

\subsection{Model Validation}

The burr formation model was validated through a simulation using the same tool and process parameters as those in [12]. All steps of the model were executed with a Dell Inspiron laptop with an Intel i5-4210U processor, in Matlab R2017b. The program inputs were the material, tool, and drilling properties necessary to calculate the following equations (described in Appendix A, Section A.1): normal rake angle, eq. (A.9); the relative radius, eq. (A.6); the dynamic rake angle, eq. (A.8); and the initial thickness of deforming material, eq. (3.9), which culminated in calculating the final burr height and thickness, eqs. (3.2) and (3.3). 
Table 3.2 Simulation material specification

\begin{tabular}{|c|c|c|c|}
\hline Material & $\begin{array}{c}\text { Yield Strength } \\
\text { (MPa) }\end{array}$ & $\begin{array}{c}\text { Tensile Strength } \\
\text { (MPa) }\end{array}$ & $\begin{array}{c}\text { \% Reduction of } \\
\text { Area }^{1}\end{array}$ \\
\hline AISI 304L & 281.6 & 620.7 & 72 \\
\hline AISI 4118 & 389.5 & 580.8 & 70 \\
\hline
\end{tabular}

Table 3.3 Simulation drill bit specifications

\begin{tabular}{|c|c|c|}
\hline Material & $\begin{array}{c}\text { Drill Diameter } \\
{[\mathrm{mm}]}\end{array}$ & Point Angle [deg] \\
\hline \multirow{2}{*}{ AISI 304L } & $1.984(5 / 64 \mathrm{in})$ & 135 \\
\cline { 2 - 3 } & $3.968(5 / 32 \mathrm{in})$ & 135 \\
\hline \multirow{2}{*}{ AISI 4118 } & 4 & 135 \\
\cline { 2 - 3 } & 7 & 135 \\
\hline
\end{tabular}

The additional variable that needed consideration as an input variable, not specifically focused on in [12], was the number of segments the drill cutting edge was to be divided into. The value affected the relative radius and consequently the dynamic rake angle. The value determined the fidelity with which the final burr heights and thicknesses were calculated. Test trials with increasing segment values were run for each material and drill diameter to determine the number of segments that would deliver consistent burr height and thickness values. The change observed was significant below 2,000 segments. Between 2,000 and 4,000 segments, and subsequently between 5,000 and 15,000 segments, the difference between height and thickness values was less than $0.001 \mathrm{~mm}$, whereas between 500 and 2,000 it was $0.016 \mathrm{~mm}$.

\footnotetext{
$1 \%$ reduction of area is a measure of material ductility based on a tensile test, indicating the ability of a material to be elongated in tension [57]. It is the proportional reduction of the cross-sectional area of a tensile test piece measured following fracture occurrence:

$$
\% R A=\frac{\text { original cross section area }- \text { minimum final area }}{\text { original cross section area }}
$$
}


Fig. 3.7 shows the burr height value progress towards consistency with the increased number of segments.

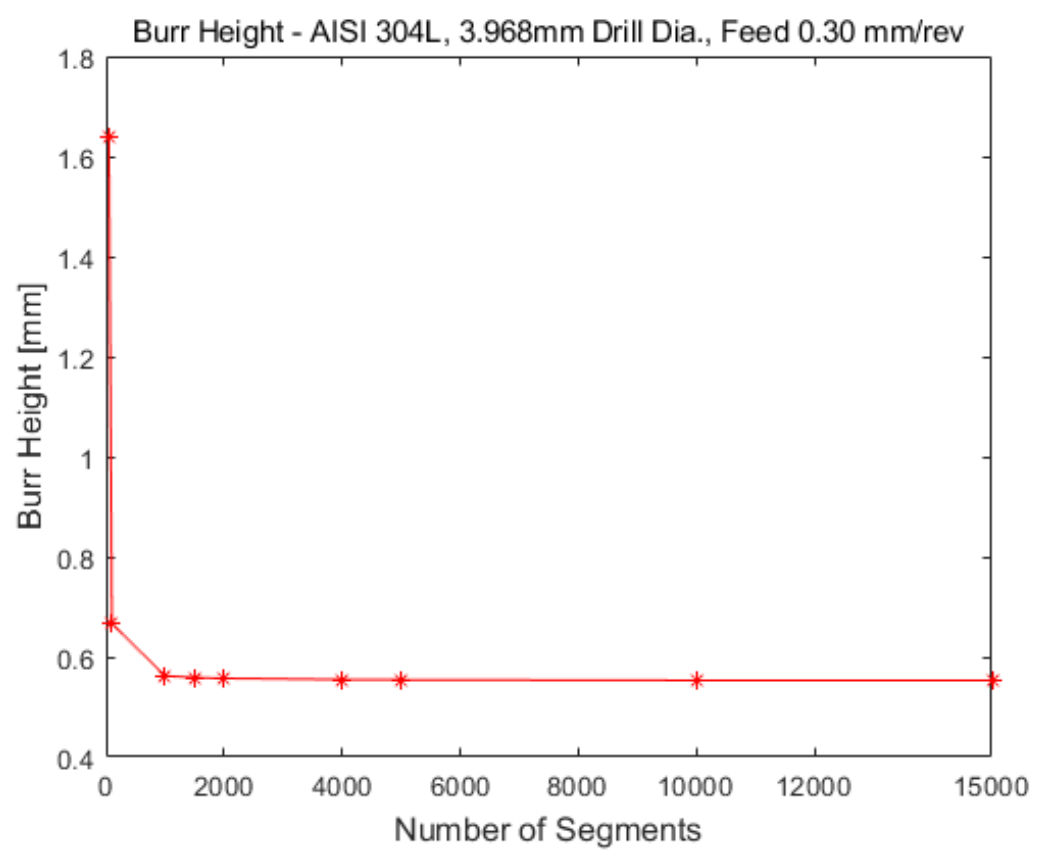

Fig. 3.7 Burr height values variance with increasing N-value of segments of AISI 304L, drilled with $3.968 \mathrm{~mm}$ dia. drill bit at 0.30 $\mathrm{mm} / \mathrm{rev}$

The simulation delivered results in alignment with those presented in [12]. Plots of these simulations can be found in Appendix B. There are four groups of plots in total, which can be grouped into two subsets. The first set, Fig. B.1 and Fig. B.2, are plots of burr heights and thicknesses with respect to a range of feeds for AISI $304 \mathrm{~L}$ and AISI 4118 , where a single point angle of $135^{\circ}$ was held constant. For this set of data, a gradual growth in burr height and thickness was observed for each drill bit diameter and for each material. A doubling of the diameter of the drill bit for AISI 304L, from 1.984 to $3.968 \mathrm{~mm}$, led to an increase in burr height of $.10 \mathrm{~mm}$ at the highest feed of $.30 \mathrm{~mm} / \mathrm{rev}$. The same degree of increase was noted for burr thickness for the same material. A similar pattern was exhibited by values calculated for AISI 4118.

The second group of data, Fig. B.3 and Fig. B.4 represent burr height and thickness growth with respect to a range of point angles, where a single feed of $.08 \mathrm{~mm} / \mathrm{rev}$ was held constant. In [12], one result was shown for this particular set of data, as feed impact was at issue for their study, and their tests included a drill bit with a single point angle. The authors in [12] calculated results for burr heights and thicknesses using AISI 304L characteristics for a range of point angles from $0^{\circ}$ to approximately $170^{\circ}$ and found a sudden increase in burr height at a point angle of approximately $150^{\circ}$. An additional set of burr heights 
and thicknesses was calculated as part of the work in this chapter for AISI 4118 with respect to the point angle, for a range of point angles from $0^{\circ}$ to $160^{\circ}$. The burr height and thickness model described in this chapter was already under consideration for modification and extended applications, so analytically evaluating its consistency for a second material was justifiable. Burr behaviour with respect to a range of point angles demonstrated stability and similar performance to that with respect to feed. As the point angle increased, the burr heights and thicknesses increased correspondingly. For AISI 4118, a $7 \mathrm{~mm}$ drill bit diameter for each material led to slight overall growth in both burr height and thickness, as compared to burrs generated with a $4 \mathrm{~mm}$ drill bit. The steadiness of the simulation data provided reassurance of the appropriateness of using this model for further investigations of drilling burr height and thickness patterns. Examining simulated burr growth patterns for a range of feeds and point angles at this point in the research was consequential, as the plan for the next stage of research was to explore burr height and thickness sensitivity with respect to variances in tool and process parameters.

\subsection{Summary and Application in Subsequent Work}

The model so far presented in this chapter describes a burr in terms of burr height and thickness, relating burr formation to the drilling thrust force generated from cutting. This chapter has shown the complexity of the burr height and thickness model and how it can be more applicable to manufacturing engineers. The burr height and thickness model comprises a number of key factors including part material property, tool geometry, and drilling parameters. Acknowledging the comprehensive value of this model, and that the key drilling factors considered with in it will vary in day-to-day drilling operations, was the first step in the decision to expand on this burr height and thickness formation model.

This led to the next step, expansion of this model to develop a sensitivity matrix for the purpose of studying the effects of variation in vital drilling parameters on burr formation.

\subsection{Sensitivity Analysis of Burr Formation}

The objective of the next stage of the research was to perform burr sensitivity analysis to establish a range where the burr was least sensitive to growth with respect to geometry and process parameters. Drilling burr formation and associated process parameters have been amply studied in the past, yet the 
details of gradual changes occurring in burr height and thickness growth have not been examined adequately. It is, therefore, warranted to examine burr height sensitivity resulting from progressing changes in critical machining and tooling parameters. Being able to clearly identify the relationship between factors affecting burr height and thickness sensitivities was intended to establish parameter ranges where a burr exhibits heightened or lowered sensitivity. The intention was for sensitivity to serve as a prescription for parameter ranges for machinists, ensuring minimal burr growth during drilling. Awareness of these ranges would then allow the application of these ranges by combining parameter range and proper tool selection for planning drilling operations. Staying within low sensitivity ranges would ensure minimal burr growth during drilling.

To examine burr height and thickness sensitivity, the burr formation model presented so far was transformed using a Jacobian by differentiating the model and effectively calculating the tangent of the model with respect to the corresponding feeds and point angles.

\subsubsection{Redefining Burr Height and Thickness}

In preparation for a Jacobian transformation, burr height and thickness models outlined in [12] and described in sections 3.2-3.4, expanded on in the first part of this chapter, are redefined as follows, starting with burr height, eq. (3.2), [12, eq. (31)], redefined as $H_{1}$ and $H_{2}$ :

$$
H=H_{1} \cdot H_{2}
$$

where

$$
\begin{gathered}
H_{1}=t_{0} \\
H_{2}=\sin p \exp \left\{\frac{\sqrt{3}}{2} \ln \left(\frac{100}{100-\% \text { R.A. }}\right)\right\}
\end{gathered}
$$


Burr thickness, eq. (3.3) [12, eq. (31)] redefined as $T_{1}$ and $T_{2}$ :

$$
T=T_{1} \cdot T_{2}
$$

where

$$
\begin{gathered}
T_{1}=t_{0} \\
T_{2}=\sin p \tan p
\end{gathered}
$$

\subsection{Jacobian Transformation}

In order to study the dominant drilling parameters, feed, and point angle, a Jacobian transformation was applied to the final burr height and thickness models. As described in Section 2.4, feed and point angle were selected based on existing research, which found these two parameters to have considerable influence on burr formation. The results in [11], which examined burr formation in drilling of 2024-T351, showed that both feed rate and point angle have the most significant roles in burr formation. The authors of [20] concluded that feed and point angle were among the top controllable parameters for optimizing burr height. The work described in [14] showed that the selection of feed and point angle values has an appreciable effect on minimizing burr height. Considering these three studies, feed and point angle were used in the Jacobian transformation because they are key drilling parameters that have been shown to contribute to both burr growth and minimization.

Employing the Jacobian showed the best non-linear approximation to the differentiable function of burr height and thickness near a given point, indicating the direction in the input space in which the burr height and thickness model increased once drilling and burr formation parameters were inputted. Burr height and deforming material thickness are functions of $f$ and $p$. The Jacobian was therefore applied to burr height and thickness as follows, starting with the first layer of derivation: 


$$
\left[\begin{array}{l}
\Delta H \\
\Delta T
\end{array}\right]=J\left[\begin{array}{l}
\Delta f \\
\Delta p
\end{array}\right]
$$

where

$$
J=\frac{\partial(H, T)}{\partial(f, p)}=\left[\begin{array}{ll}
\frac{\partial H}{\partial f} & \frac{\partial H}{\partial p} \\
\frac{\partial T}{\partial f} & \frac{\partial T}{\partial p}
\end{array}\right]
$$

\subsubsection{Jacobian Transformation Applied to Burr Height}

Given the above redefinition of burr height and thickness into two parts, the focus was first placed on burr height differentiation. This expansion is like a Taylor expansion, a function of feed and point angle. This is the second layer of derivation:

$$
\begin{gathered}
\Delta H=\left[\begin{array}{c}
\frac{\partial H}{\partial f} \frac{\partial H}{\partial p}
\end{array}\right]\left[\begin{array}{l}
\Delta f \\
\Delta p
\end{array}\right]=\frac{\partial H}{\partial f} \Delta f+\frac{\partial H}{\partial p} \Delta p \\
\frac{\partial H}{\partial f}=\left[\begin{array}{ll}
\frac{\partial H_{1}}{\partial f} & \frac{\partial H_{2}}{\partial f}
\end{array}\right]\left[\begin{array}{l}
H_{2} \\
H_{1}
\end{array}\right] \\
\frac{\partial H}{\partial p}=\left[\begin{array}{ll}
\frac{\partial H_{1}}{\partial p} & \frac{\partial H_{2}}{\partial p}
\end{array}\right]\left[\begin{array}{l}
H_{2} \\
H_{1}
\end{array}\right]
\end{gathered}
$$

\subsubsection{Jacobian Transformation Applied to Burr Thickness}

The Jacobian was applied similarly to burr thickness, continuing the second layer of derivation:

$$
\begin{gathered}
\Delta T=\left[\frac{\partial T}{\partial f} \frac{\partial T}{\partial p}\right]\left[\begin{array}{l}
\Delta f \\
\Delta p
\end{array}\right]=\frac{\partial T}{\partial f} \Delta f+\frac{\partial T}{\partial p} \Delta p \\
\frac{\partial T}{\partial f}=\left[\begin{array}{ll}
\frac{\partial T_{1}}{\partial f} & \frac{\partial T_{2}}{\partial f}
\end{array}\right]\left[\begin{array}{l}
T_{2} \\
T_{1}
\end{array}\right] \\
\frac{\partial T}{\partial p}=\left[\begin{array}{ll}
\frac{\partial T_{1}}{\partial p} & \frac{\partial T_{2}}{\partial p}
\end{array}\right]\left[\begin{array}{l}
T_{2} \\
T_{1}
\end{array}\right]
\end{gathered}
$$




\subsubsection{Continued Derivation of Burr-Height and Thickness Relationships}

To examine burr height and thickness sensitivity, burr height (eq. [3.2]) and burr thickness (eq. [3.3]) relationships already transformed with a Jacobian were differentiated with respect to feed and point angle. The process began with differentiating the initial thickness of deforming material, $t_{0}$, eq. (3.15), as a function of $f$ and $p$. The process was identical for both burr height and burr thickness relations, and initial chip thickness. First, burr height and thickness were differentiated with respect to feed; initial thickness of deforming material was differentiated to incorporate $\partial \mathrm{H} / \partial \mathrm{f}$ and $\partial \mathrm{T} / \partial \mathrm{f}$ :

$$
\begin{aligned}
t_{0}=F\left(J_{1}, J_{2}, J_{3}\right)=H_{1} & =T_{1} \rightarrow \frac{\partial H_{1}}{\partial f}=\frac{\partial T_{1}}{\partial f} \\
\frac{\partial H_{2}}{\partial f} & =0 \\
\frac{\partial T_{2}}{\partial f} & =0
\end{aligned}
$$

Similarly, for point angle differentiation, burr height and thickness were differentiated with respect to the point angle:

$$
\begin{gathered}
t_{0}=F\left(J_{1}, J_{2}, J_{3}\right)=H_{1}=T_{1} \rightarrow \frac{\partial H_{1}}{\partial p}=\frac{\partial T_{1}}{\partial p} \\
\frac{\partial H_{2}}{\partial p}=t_{0} \cos p \exp \left\{\frac{\sqrt{3}}{2} \ln \left(\frac{100}{100-\% R \cdot A .}\right)\right\} \\
\frac{\partial T_{2}}{\partial p}=t_{0} \cos p \tan p+\sin p \sec p^{2}
\end{gathered}
$$

Next, burr height and chip thickness were differentiated with respect to feed and point angle, creating the third layer of derivation:

$$
\begin{aligned}
& \frac{\partial H_{1}}{\partial f}=\frac{\partial T_{1}}{\partial f}=\frac{\partial t_{0}}{\partial f}=\left[\frac{\partial F}{\partial J_{1}} \frac{\partial J_{1}}{\partial f}\right]+\left[\frac{\partial F}{\partial J_{2}} \frac{\partial J_{2}}{\partial f}\right]+\left[\frac{\partial F}{\partial Z} \frac{\partial J_{2}}{\partial f}\right] \\
& \frac{\partial H_{1}}{\partial p}=\frac{\partial T_{1}}{\partial p}=\frac{\partial t_{0}}{\partial p}=\left[\frac{\partial F}{\partial J_{1}} \frac{\partial J_{1}}{\partial p}\right]+\left[\frac{\partial F}{\partial J_{2}} \frac{\partial J_{2}}{\partial p}\right]+\left[\frac{\partial F}{\partial J_{3}} \frac{\partial J_{3}}{\partial p}\right]
\end{aligned}
$$


The implicit differentiation of eq. (3.32) with respect to feed was achieved by differentiating eqs. (3.103.13):

$$
\begin{aligned}
& \frac{\partial F}{\partial J_{1}} \frac{\partial J_{1}}{\partial f}=\left\{\left[-\frac{1}{2 J_{1}^{2}} \frac{\partial J_{1}}{\partial f}\right]\left(-J_{2}+\sqrt{J_{2}^{2}-4 J_{1} J_{3}}\right)\right\}+\left\{\frac{1}{2 J_{1}}\left[\left(\frac{1}{2 \sqrt{J_{2}{ }^{2}-4 J_{1} J_{3}}}\right) \cdot\left(-4 J_{3}\right) \frac{\partial J_{1}}{\partial f}\right]\right\} \\
& \frac{\partial F}{\partial J_{2}} \frac{\partial J_{2}}{\partial f}=\left\{\frac{1}{2 J_{1}}\left[\left(-1+\left(\frac{1}{2 \sqrt{J_{2}{ }^{2}-4 J_{1} J_{3}}}\right) 2 J_{2}\right) \cdot \frac{\partial J_{2}}{\partial f}\right]\right\} \\
& \frac{\partial F}{\partial J_{3}} \frac{\partial J_{3}}{\partial f}=\left\{\frac{1}{2 J_{1}}\left[\left(\frac{1}{2 \sqrt{J_{2}{ }^{2}-4 J_{1} J_{3}}}\right) \cdot\left(-4 J_{1} \frac{\partial J_{3}}{\partial f}\right)\right]\right\}
\end{aligned}
$$

For differentiation with respect to the point angle, recalling eqs. (3.10-3.13), the deforming material thickness eq. (3.9) was differentiated with respect to the point angle:

$$
\frac{\partial t_{0}}{\partial p}=\left[\frac{\partial F}{\partial J_{1}} \frac{\partial J_{1}}{\partial p}\right]+\left[\frac{\partial F}{\partial J_{2}} \frac{\partial J_{2}}{\partial p}\right]+\left[\frac{\partial F}{\partial J_{3}} \frac{\partial J_{3}}{\partial p}\right]
$$

Similarly to eqs. (3.36)-(3.38), it is necessary to perform implicit differentiation of Eqs. (3.10-3.13) with respect to the point angle:

$$
\begin{aligned}
& \frac{\partial F}{\partial J_{1}} \frac{\partial J_{1}}{\partial p}=\left\{\left[-\frac{1}{2 J_{1}{ }^{2}} \frac{\partial J_{1}}{\partial p}\right]\left(-J_{2}+\sqrt{J_{2}^{2}-4 J_{1} J_{3}}\right)\right\}+\left\{\frac{1}{2 J_{1}}\left[\left(\frac{1}{2 \sqrt{J_{2}^{2}-4 J_{1} J_{3}}}\right) \cdot\left(-4 J_{3} \frac{\partial J_{1}}{\partial p}\right)\right]\right\} \\
& \frac{\partial \mathrm{F}}{\partial J_{2}} \frac{\partial J_{2}}{\partial \mathrm{p}}=\left\{\frac{1}{2 J_{1}}\left[\left(-1+\left(\frac{1}{2 \sqrt{J_{2}^{2}-4 J_{1} J_{3}}}\right) 2 J_{2}\right) \cdot \frac{\partial J_{2}}{\partial \mathrm{p}}\right]\right\} \\
& \frac{\partial F}{\partial J_{3}} \frac{\partial J_{3}}{\partial p}=\left\{\frac{1}{2 J_{1}}\left[\left(\frac{1}{2 \sqrt{J_{2}{ }^{2}-4 J_{1} J_{3}}}\right) \cdot\left(-4 J_{1} \frac{\partial J_{3}}{\partial p}\right)\right]\right\}
\end{aligned}
$$

\subsubsection{Subcomponent Differentiation with Respect to Feed}

The individual differentiated components of the deforming material thickness, in each case consisting of the function first differentiated with respect to $J_{1}, J_{2}$, or $J_{3}$ components and then each of eqs. (3.103.13), were differentiated with respect to the feed, creating the fourth layer of derivation, starting with eq. (3.10): 


$$
\begin{gathered}
J_{1}=\frac{3}{4} \pi \sin p^{3} \cos p+\frac{1}{2} \pi\left(\frac{\pi}{2}-p\right) \\
\frac{\partial J_{1}}{\partial f}=0
\end{gathered}
$$

Next, the differentiation of eq. (3.11) resulted in:

$$
\begin{gathered}
J_{2}=\frac{3}{4} \pi R \sin p \ln \left(\frac{1}{\sin p}\right)-\frac{\sqrt{2}}{9} f \sigma_{u} \cdot \Phi \\
\frac{\partial J_{2}}{\partial f}=-\frac{\sqrt{2}}{9} \sigma_{u} \cdot \Phi-\frac{\sqrt{2}}{9} f \sigma_{u} \cdot\left(\frac{\partial \Phi}{\partial \mathrm{f}}\right)
\end{gathered}
$$

Finally, differentiating eq. (3.12) yielded:

$$
\begin{gathered}
J_{3}=-\frac{\sqrt{2}}{9} \frac{f}{\tan p} R \sigma_{u} \cdot \Phi \\
\frac{\partial J_{3}}{\partial f}=-\frac{\sqrt{2}}{9} \frac{1}{\tan p} R \sigma_{u} \cdot \Phi-\frac{\sqrt{2}}{9} \frac{f}{\tan p} R \sigma_{u} \cdot\left(\frac{\partial \Phi}{\partial \mathrm{f}}\right)
\end{gathered}
$$

Note: due to its complexity and length, the detailed differentiation of $\partial \Phi / \partial \mathrm{f}$ can be found in Appendix C.

\subsubsection{Subcomponent Differentiation with Respect to Point Angle}

Continuing with the Jacobian transformation, burr height and thickness were differentiated with respect to the point angle, resulting in $\partial \mathrm{H} / \partial \mathrm{p}$ and $\partial \mathrm{T} / \partial \mathrm{p}$. Similar to eqs. (3.43)(3.45), the individual components of $t_{0}$ were differentiated with respect to the point angle, starting with eq. (3.10):

$$
\begin{gathered}
J_{1}=\frac{3}{4} \pi \sin p^{3} \cos p+\frac{1}{2} \pi\left(\frac{\pi}{2}-p\right) \\
\frac{\partial J_{1}}{\partial p}=\frac{3}{4} \pi(3) \sin ^{2} p \cos ^{2} p-\frac{3}{4} \pi(\sin p)^{4}-\frac{1}{2} \pi
\end{gathered}
$$


Eq. (3.11) differentiated with respect to the point angle yielded:

$$
\begin{array}{r}
J_{2}=\frac{3}{4} \pi R \sin p \ln \left(\frac{1}{\sin p}\right)-\frac{\sqrt{2}}{9} f\left(\frac{\sigma_{u}}{\sigma_{y}}\right) \cdot \Phi \\
\frac{\partial J_{2}}{\partial p}=\frac{3}{4} \pi R \cos p \ln \left(\frac{1}{\sin p}\right) \cdot \Phi-\frac{3}{4} \pi R \sin p\left(\frac{\cos p}{\sin p}\right)-\frac{\sqrt{2}}{9} f \sigma_{u} \cdot\left(\frac{\partial \Phi}{\partial p}\right) \\
\frac{\partial J_{2}}{\partial p}=\frac{3}{4} \pi R \cos p\left(\ln \left(\frac{1}{\sin p}\right)-1\right)-\frac{\sqrt{2}}{9} f \sigma_{u} \cdot\left(\frac{\partial \Phi}{\partial \mathrm{p}}\right)
\end{array}
$$

Finally, eq. (3.12) differentiated with respect to the point angle resulted in:

$$
\begin{array}{r}
J_{3}=-\frac{\sqrt{2}}{9} f \sigma_{u} \frac{R}{\tan p} \cdot \Phi \\
\frac{\partial J_{3}}{\partial p}=\frac{\sqrt{2}}{9} \frac{f}{\tan ^{2} p} \sec ^{2} p R \sigma_{u} \cdot \Phi-\frac{\sqrt{2}}{9} \frac{f}{\tan p} R\left(\frac{\sigma_{u}}{\sigma_{y}}\right) \cdot\left(\frac{\partial \Phi}{\partial p}\right) \\
\frac{\partial J_{3}}{\partial \mathrm{p}}=\frac{\sqrt{2}}{9} \frac{\mathrm{f}}{\tan \mathrm{p}} \operatorname{R} \sigma_{\mathrm{u}}\left(\left(\frac{1}{\tan \mathrm{p}} \sec ^{2} \mathrm{p} \cdot \Phi\right)-\left(\frac{\partial \Phi}{\partial \mathrm{p}}\right)\right)
\end{array}
$$

Note: Due to its complexity and length, the detailed differentiation of $\partial \Phi / \partial \mathrm{p}$ can be found in Appendix C.

\subsection{Evaluation}

\subsubsection{D Evaluation of Sensitivity Analysis}

The burr sensitivity model was evaluated in a simulation using the same material characteristics and process parameters as those in Section 3.5 (see Tables 3.2 and 3.3). Burr height, thickness, and their associated rates of change for drilling with AISI 304L and AISI 4118 with 1.984-mm and 4-mm diameter drill bits, respectively, are presented in Fig. 3.8 and Fig. 3.9. Burr growth behaviour was similar for height and thickness simulations, using larger diameters within each material. The burr sensitivity model results were calculated with Matlab R2017b. The program inputs were material, tool, and drilling properties necessary to calculate final burr height and thickness and all the rates of change of burr height and thickness with respect to feed and point angle.

The simulation for both materials demonstrated steady rates of change for the parameters examined. For each material and drill bit, there were two groups of results. The first showed the burr height and thickness growth versus a range of feeds, where the point angle was held constant, and the growing 
rates of change of burr heights and thicknesses with respect to a range of feed values. The second set showed burr height and thickness growth versus a range of point angles, where a single feed value was held constant, and the rates of change of burr heights and thicknesses with respect to a range of point angles. In each material's case, two similar patterns emerged. Burr height and thickness increased gradually with feed increase. Conversely, the rate of change of burr height and thickness with respect to feed decreased as the feed increased. When looking at burr height and thickness trends with respect to the point angle, burr height and thickness increased as the point angle increased. The rate of change of burr height and thickness also increased as the point angle increased. Therefore, the trend for the point angle was the same for both burr growth and the rate of change of burr growth.

The first group of 2D plots for AISI 304L, 1.984-mm diameter are shown in Fig. 3.8 and Fig. 3.9. The remainder of the graphs can be found in Appendix D, Section D.1.
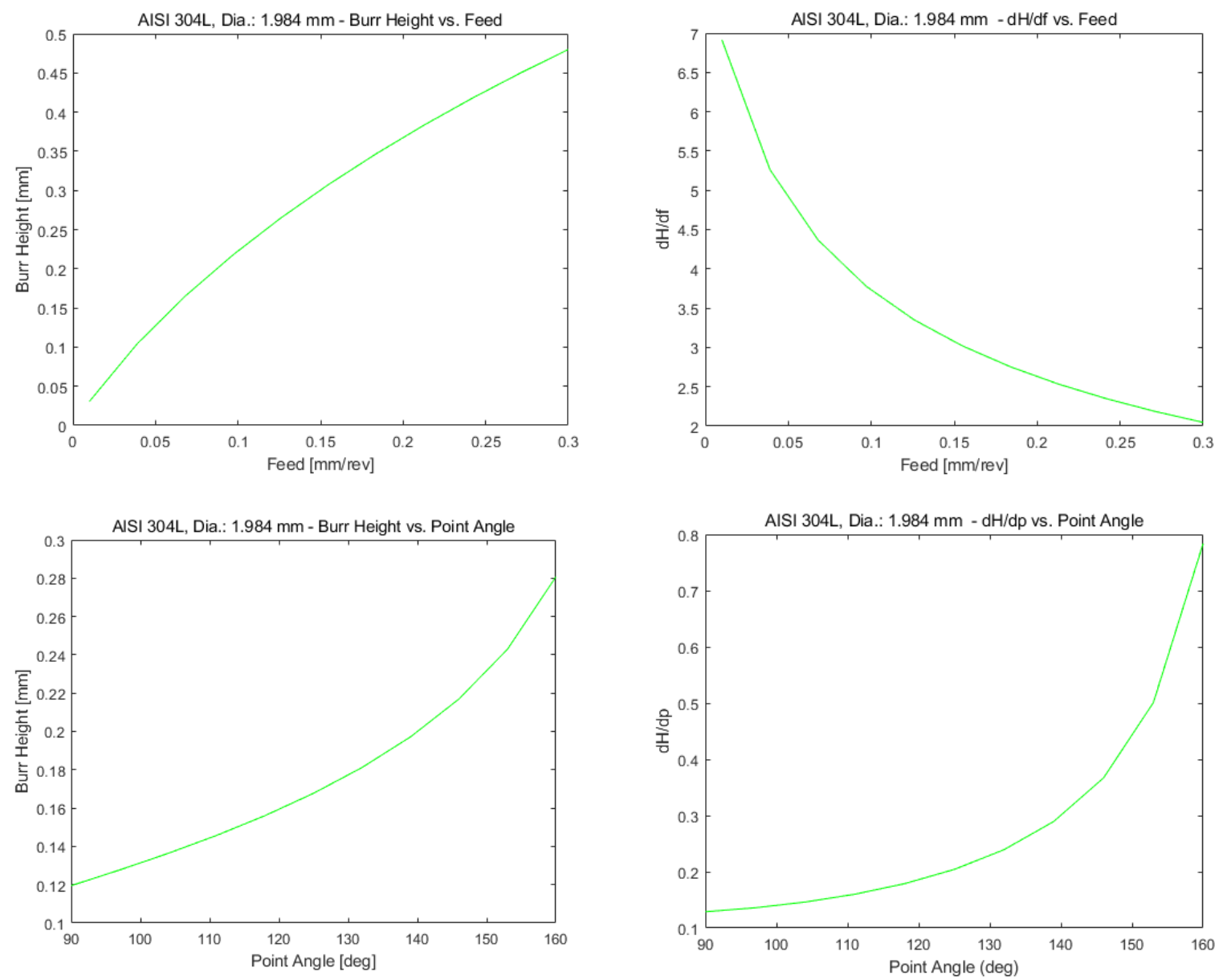

Fig. 3.8 Burr height and rate of change of burr height for AISI 304L, 1.984-mm dia. drill bit 

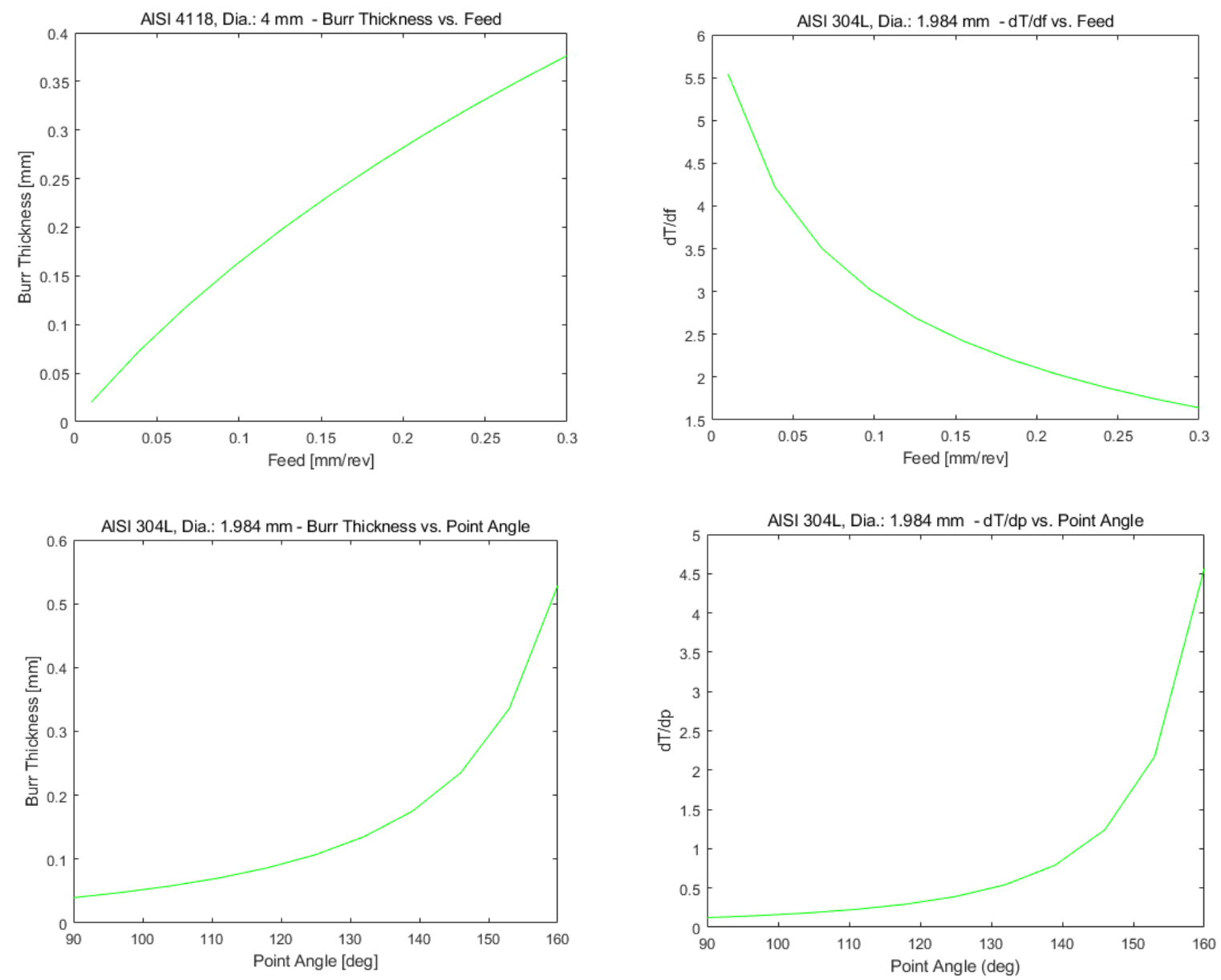

Fig. 3.9 Burr thickness and rate of change of burr thickness for AISI 304L, 1.984-mm dia. drill bit

The opposing trends for rates of change of feed versus rates of change of point angles indicated that the newly developed burr sensitivity model could lead to the establishment of a range of feed and point angle values that would enable the formulation of a minimal burr height thickness and a minimal rate of change of burr height and thickness.

While the 2D plots showed only two sets of data at one time (i.e., a burr height and thickness or the rate of change of burr height and thickness) plotted against a single drilling parameter (i.e., feed or point angle), a 3D plot allowed for further analysis.

\subsubsection{D Evaluation of Sensitivity Analysis}

A 3D surface plot evaluation was conducted in an attempt to find the abovementioned ranges of feeds and point angles. Burr height and thickness and the rates of change of burr height and thickness were plotted as functions of feed and point angle. Similarly, burr height was examined through the use of 3D plots, in [14] and [44]. The objective was to capitalize on the opposing trends of burr growth with 
respect to feed and the rates of change to enable the location of a region in the 3D plot where burr height and thickness and the rates of change of burr height and thickness were minimal. The first set of 3D surface plots is shown below in Fig. 3.10 for AISI 304L, 1.984-mm diameter drill bit. Surface plots for AISI 304L 3.968-mm diameter drill bit and for AISI 4118 4-mm and 7-mm diameter drill bits are in Appendix D.

Upon detailed scrutiny, the surface plots did not reveal a zone of minimal burr height or thickness, nor of areas of minimal rates of change of burr height or thickness. Therefore, the 3D plots were not indicative of the desired point angle or feed range. The plots consistently showed that a minimal burr and a minimal rate of change were achievable only at minimum feeds and point angles. Minimum feeds and point angles are not practical for efficient drilling to occur. This causes worse hole quality and shorter tool life. Each work material has a designated point angle, which ensures the highest hole quality and longest drill bit longevity [29]. Thus, the smallest point angle is not necessarily the one delivering the most favourable drill bit life and hole quality. Appropriate feeds are generally determined based on drill bit diameter size and drill bit material [29], [31]. Variances in the point angle and feed ranges enabling minimal burr height and thickness would still need to follow established drilling tool and process parameter selection practices.

The two varying sets of trends observed in 2D plots, described in Section 3.9.1, between burr height and thickness growth and rates of change of burr height and thickness with respect to feed and point angle were the dominant contributors to the 3D surface plot results.

Based on the methods used in [45], it was decided that optimization was the next step. In [45], the authors first performed a factorial experiment to calculate the impact of drilling process parameters with different tool types and tool-wear conditions on a set of output variables: thrust forces and delamination behaviour of carbon fibre reinforced polymer (CFRP) laminates. The authors subsequently carried out an optimization, hoping to find a unique solution of cutting parameter values that would minimize all output variables. When this did not deliver the hoped-for result, they chose to conduct a multi-objective optimization with weights to obtain a complete optimum solution: a range of cutting parameter values to minimize the drilling output variables.

In pursuit of a point angle and feed settings that would achieve burr minimization in growth and rate of change of burr height and thickness, the burr sensitivity model was optimized. The optimization is discussed in Section 3.10. 

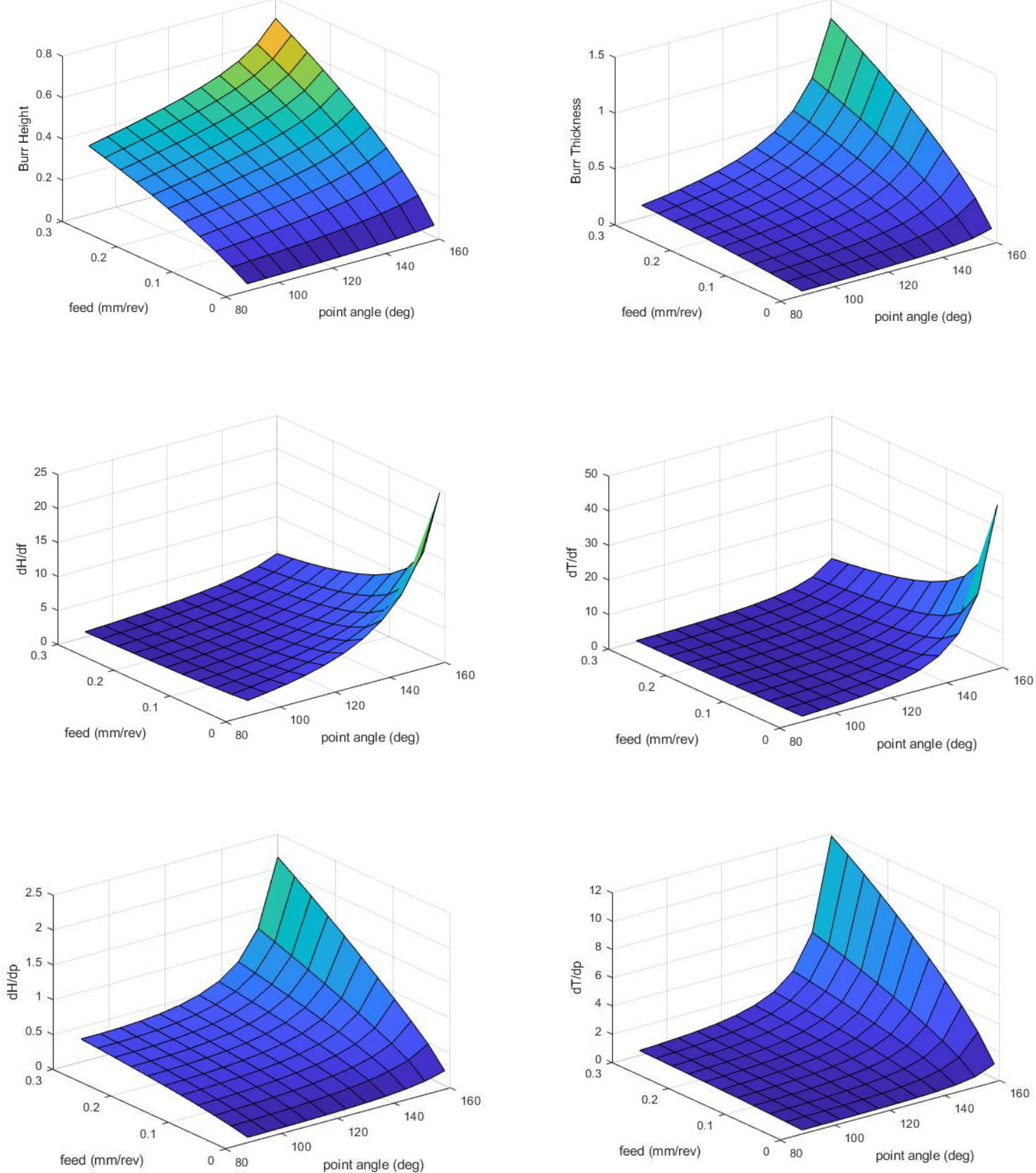

Fig. 3.10 AISI 304L 1.984-mm dia. - surface plots of burr height, thickness, and respective rates of change of height and thickness vs. point angle and feed

\subsection{Optimization}

The goal in examining burr height and thickness sensitivity was to obtain an optimal set of feed and point angle values for ductile materials to consistently deliver the lowest burr height and thickness values together with minimal burr height and thickness rates of change, minimal burr height and the 
thickness rate of change demonstrating minimal sensitivity. The desired set of feed and point angle values that would deliver a minimal burr and the lowest rate of change of burr height and thickness with respect to feed and the point angle was not immediately obvious from the set of plotted values in either the $2 \mathrm{D}$ or $3 \mathrm{D}$ representation.

By formulating the sensitivity analysis as an optimization problem it was attempted to establish feeds and point angles producing a minimal burr with the lowest change in growth. The intent was to find a region where both the height $H$ and thickness $T$ and their rates of change with respect to feed and point angle, $\partial H / \partial p, \partial H / \partial f, \partial T / \partial p$, and $\partial T / \partial f$, were minimal.

Expressed mathematically, the goal was to find $\boldsymbol{x}=[f, p]$ such that $H$ and $T$ and their associated rates of change were all minimized.

The optimal set of feed and point angles was defined as follows:

$$
\begin{gathered}
x=[f, p] \\
x^{*}=\min F(x)
\end{gathered}
$$

An objective function was constructed to find the set of feed and point angle values, $x^{*}$, that would deliver the minimal and most stable burr height or thickness. The objective function was as follows:

$$
F=w_{1} H+w_{2}\left[\left(\frac{\partial H}{\partial f} \cdot \Delta f \cdot e_{1}\right)+\left(\frac{\partial H}{\partial p} \cdot \Delta p \cdot e_{2}\right)\right]
$$

where $F$ is the objective function, calculating the combination of burr height, thickness, and associated rates of change. The objective had two terms. The first term was always either the burr height or thickness and therefore a component of Dornfeld's model. The second term was the rate of change of burr height or thickness with respect to feed and/or point angle.

The other variables in the above objective function, $w$ and $e$, were the function's hyperparameters (measures to generalize the data patterns that the objective function was to generate). Hyperparamaters were incorporated in the form of weights, $w$, and efficiencies, $e$. Weight $w_{1}$ was assigned to the burr height $H, w_{2}$ was assigned to thickness $T, w_{3}$ and $w_{4}$ could be assigned to the rate of change variables, $\partial H / \partial p, \partial H / \partial f, \partial T / \partial p$, and $\partial T / \partial f$. Efficiencies $e_{1}$ and $e_{2}$ were incorporated by adding a percent efficiency to the feed and point angle, respectively. A $100 \%$ efficiency, represented by a weight of 1 , indicated ideal drilling parameters: a brand new, sharp drill bit with an unchanging point angle, and the feed delivered by the drill precisely as set, without deviations due to drill mechanism 
wear and tear. Any deviation from ideal conditions, being less than $100 \%$ and therefore $[0,1)$, indicated a state where the drill bit and feed were not performing in the ideal state. The objective with incorporating a percent efficiency was to assess burr behaviour when, for example, a drill bit was becoming worn, which altered the ideal point angle, or the drill press feeding the drill bit was no longer performing at its peak because of wear and tear. Variants of the above cost function were utilized, incorporating different weight and efficiency values and different combinations of $H, T$, and $\partial H / \partial p$, $\partial H / \partial f, \partial T / \partial p$, and $\partial T / \partial f$.

The optimization was constrained by the materials for which it was conducted: AISI 304L for 1.984-mm and 3.968-mm drill bits, and AISI 4118 for 4-mm and 7-mm drill bits. Several combinations of weight and efficiency values were attempted in the search for the best solution. A lesser weight value was always assigned to the burr height or thickness. A greater weight value was assigned to the variable sets containing the rates of change to provide enhanced visibility to normalize the magnitude of the rates of change of burr height and thickness with respect to the drilling parameters.

Table 3.4 shows a subset of the objective functions with a variety of weights assigned to the variables of burr height, thickness, and associated rates of change, and a range of efficiencies assigned to the feed and point angle. The weights were assigned without rigor because every time the optimization algorithm was run, it became obvious that regardless of the combination of weights and efficiencies applied within the objective function, the feed and point angle values returned were always the same. The objective functions were also scrutinized graphically, as presented by the sample of surface plots in Table 3.4, to examine the location of the minima. In all cases the minima presented itself at minimum parameter values, which did not lead to any novel conclusions about parameter ranges.

An example of the objective function and its plots can be seen in Table 3.4. Additional examples of objective function calculations can be found in Appendix D, Section D.3. 
Table 3.4 Objective function results

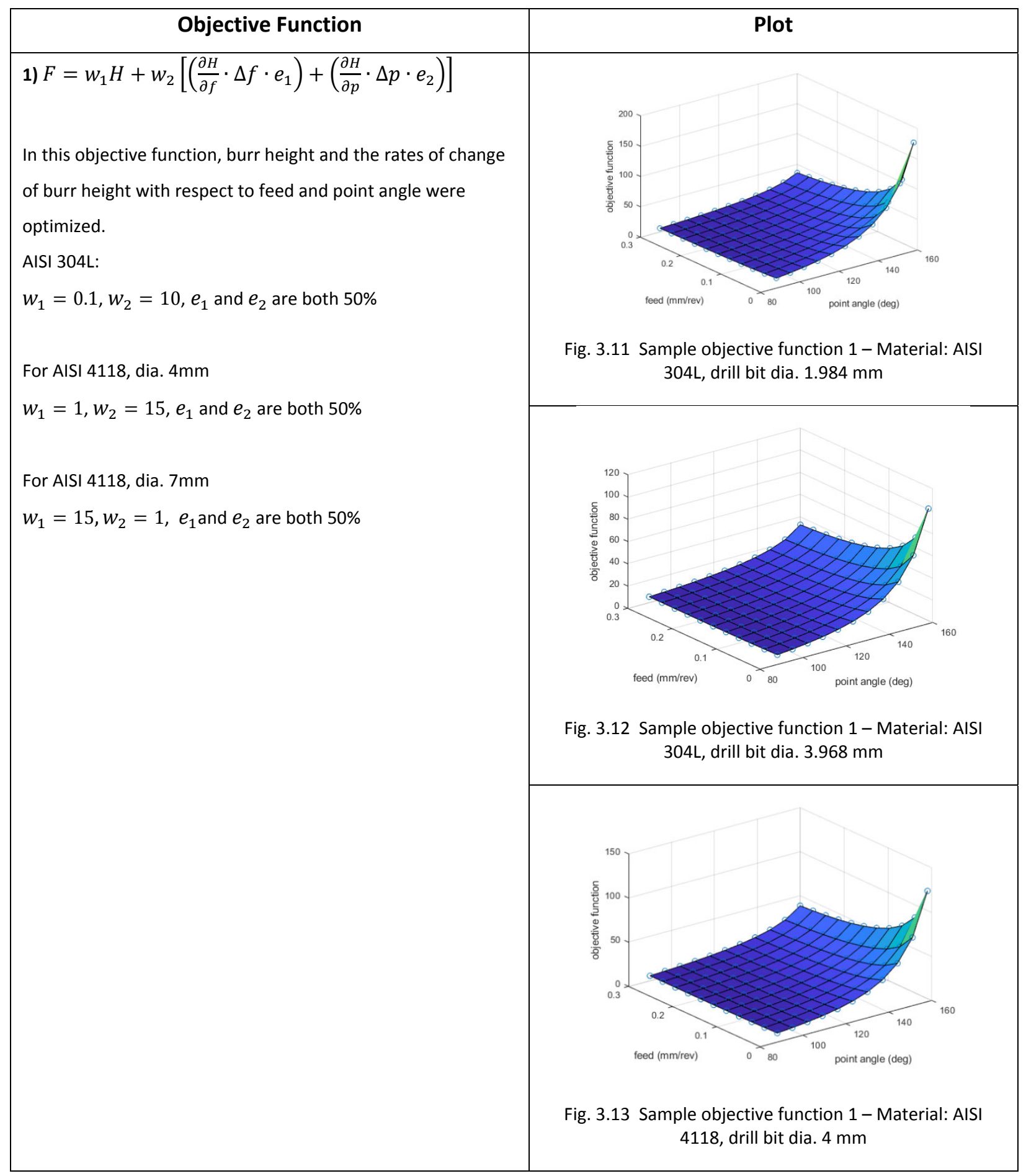




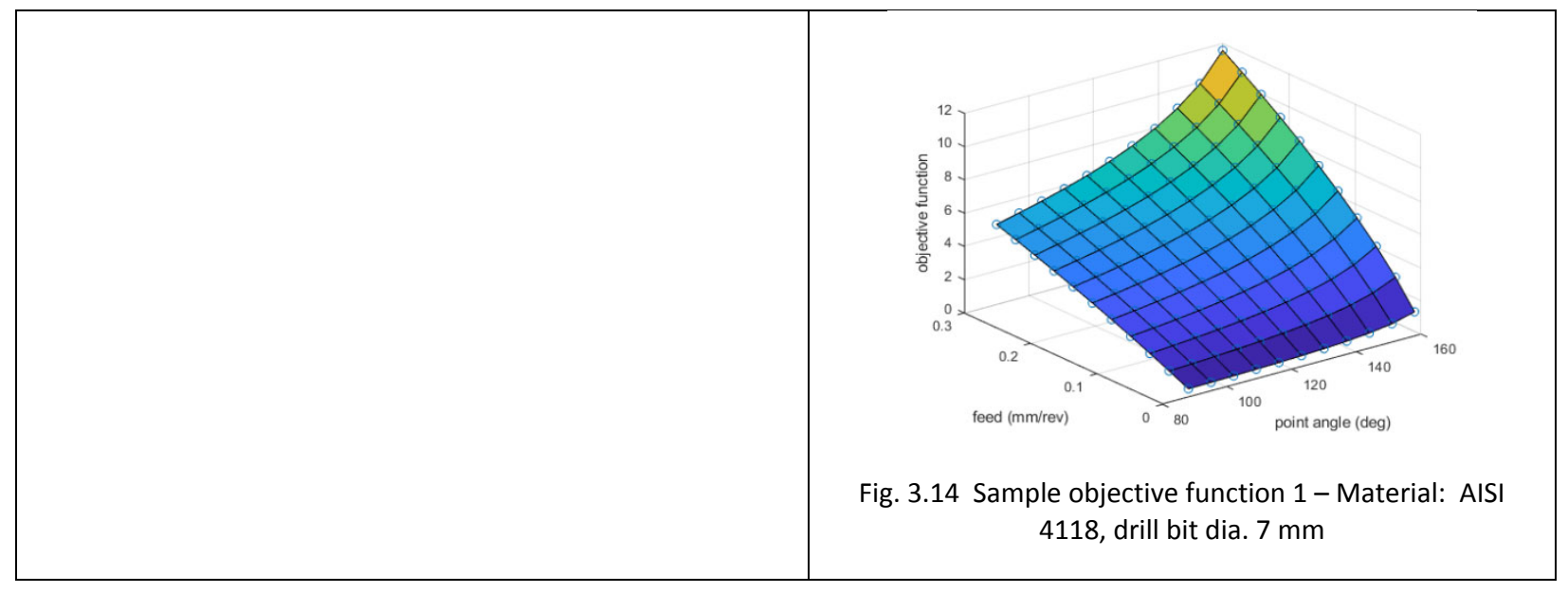

\subsection{Burr Sensitivity Model: Conclusions}

The work in this chapter was two-fold. The first stage was the formulation of a burr sensitivity model, based on the burr height and thickness model in [12], while applying a Jacobian transformation. The second stage was establishing a combination of feed and point angle in drilling that would consistently produce a minimal burr height and thickness at a minimal rate of change and subsequently be utilized as feed and point angle settings in drilling ductile materials in aerospace manufacturing.

The first stage in the burr sensitivity model introduced the rates of change of burr height and thickness with respect to the key parameters of feed and point angle.

Point angle and feed are considered to be continuous variables as components of the burr sensitivity model. With respect to this aspect, the work done in this dissertation is similar to the work in [12] and [45]. In the first study [12], the authors treated feed as a continuous variable, examining burr height and thickness growth across a range of feeds from approximately $0.03 \mathrm{~mm} / \mathrm{rev}$ to $0.25 \mathrm{~mm} / \mathrm{rev}$ in experimental and analytical results. They also treated the point angle as a continuous variable, examining the growth pattern across a continuous range of point angle values ranging from approximately $90^{\circ}$ to $170^{\circ}$.

Similarly, the researchers in [45] also utilized continuous ranges of feeds and point angles to better comprehend the impact of drilling parameters combined with a variety of tool wear conditions on woven carbon fibre reinforced polymer (CFRP) laminates. In [13] the influence of feed on burr height was studied, treating feed as a continuous variable. The authors examined the impact of feed ranging from $0.04 \mathrm{~mm} / \mathrm{rev}$ to $0.20 \mathrm{~mm} / \mathrm{rev}$ to find the optimal feed range to reduce burr height. 
Development of a sensitivity model using feed and the point angle as continuous variables is consistent with past studies.

Continuous variables allow researchers to examine burr height and thickness in their entirety instead of calculating only a small sample by using discrete feed and point angle values. Moreover, continuous variables are used to capture potential variances; when using only a set of discrete values, such variances may not become apparent.

Calculations of the new sensitivity model produced 2D representations that showed differences in burr behaviour with respect to an increasing feed and an increasing point angle. Burr height and thickness both increased with respect to an increasing feed. The rates of change of burr height and thickness with respect to feed both decreased, presenting an opposing trend for burr height and thickness growth versus the rates of change of burr height and thickness. Conversely, for the point angle, burr height and thickness both increased and their rates of change with respect to the point angle also increased.

The two varying trends between burr height and thickness growth and rates of change of burr height and thickness with respect to feed and point angle were analyzed in 2D plots. This analysis led to the formulation of 3D surface plots that were an attempt to establish a region in the parameter space where burr height and thickness and their rates of change were consistently minimal, by combining continuous ranges of the key drilling parameters (i.e., feeds and point angles) combined with the rates of change of these parameters.

Such ranges were not apparent from the 3D plots. The plot results showing that minimal feeds and point angles will result in minimal burr heights, thicknesses, and rates of change, could only have a very limited application in real-life drilling practices.

The goal of the study was to continue to search for a parameter range beyond the most obvious solution of minimum point angle and feed. Therefore, the next step was to search for optimal values of feed and point angle for the burr sensitivity model via optimization.

\subsection{Optimization Model: Conclusion}

Proceeding with optimization enabled further exploration of the burr sensitivity model. To arrive at the optimal feed and point angle values for a minimal burr height and thickness with a minimum rate of change of both, an objective function was constructed. The objective function was split into two terms, 
burr height or thickness being the first and the rates of change of burr height and/or thickness with respect to feed or point angle was the second. The first term was based on the model in [12] and the second on the new burr sensitivity model. Hyperparameters in the form of weights and efficiencies were applied to each term. The weights and efficiencies were meant to account for realistic day-to-day conditions in drilling, where tool geometry (point angle) and drilling parameters (feed) may not always be ideal due to both machine and tool wear and tear. Multiple combinations of weights and efficiencies were attempted through variants of the objective function. Merging the burr height and/or thickness, their associated rates of change, and weight and efficiency inclusion in optimization, were meant to showcase the set of feeds and point angles where these realistic conditions would manifest themselves and could be accounted for. This would enable the calculation of a minimal burr height and thickness and minimized rate of change of burr height and thickness with respect to feed and the point angle. With such a parameter setting, irrespective of the real-life machining variances, a burr could always be minimized.

Upon performing the optimization, a search all of the optimization attempts resulted in a single set of minima; this was an important result in this research. It demonstrated that the burr sensitivity approach could not completely account for drill bit and process variability. Moreover, the work done did not result in the expected solution, but it indicated where the work should lead.

The decreasing rate of change of burr height and thickness with respect to feed versus the increasing burr height and thickness with respect to feed, demonstrated the possibility for an optimal range of burr heights. This resulted in opposing trends, indicating that a low burr height or thickness and a low rate of change of burr height and thickness could be achieved.

The point angle, however, did not present such an opportunity since burr growth with respect to the point angle and the rate of change of burr growth with respect to the point angle both increased. While a gradually increasing point angle does not necessarily indicate tool wear, minimal variances in the point angle, which was treated as a continuous variable, could signify changes in the tool shape, stemming from gradual wear of the drill bit's cutting edges.

Given these observations, the work in this chapter to complete the sensitivity model, and the subsequent analysis and optimization, served as a preliminary milestone towards the next stage of research. The burr growth trend stemming from point angle changes was an indicator that tool wear could be a significant contributor to burr growth, and its impact needs to be further examined. The next 
step was, therefore, examining drilling burr growth in tandem with tool wear, which is explored in Chapter 4. 


\section{CHAPTER 4 PLOUGHING FORCE ANALYSIS AND NEW BURR FORMATION MODEL}

\subsection{Ploughing Force in Orthogonal Cutting}

In Chapter 3, in developing a burr sensitivity matrix, it was established that varying drill bit geometry stemming from wear and tear contributes to burr growth. The objective of this stage of research was to build on that work and develop a model demonstrating the relationship between drill bit wear and burr increase.

Drill bit wear is a given in the aerospace industry, considering the volume of holes drilled in each aircraft being built. Tool wear occurs gradually, with the tool material progressively being removed at the point of contact with the workpiece and at tool contact regions [34].

The drilling burr height and thickness formation model of Kim and Dornfeld [12], detailed in Chapter 3, is dependent on sharp drill bits as it does not account for tool wear. The drilling thrust force, central to the method, is assumed to act on each element of the segmented, sharp drill bit cutting edge, shearing the work material as the hole is being drilled. In any operation involving metal cutting, including drilling, tool wear is inevitable. Therefore, excluding tool wear renders the findings non-realistic for day-to-day machining.

Waldorf, DeVor, and Kapoor [15] developed a model for ploughing components of a cutting force during orthogonal cutting by estimating cutting forces. They performed the cutting operations with different sized edge tools, including ones with large cutting edge radii, that were meant to mimic worn cutting tool edges.

The model relates the percentage of ploughing forces to the size of the radius at the tool cutting edge, linking an increased cutting edge radius to a correspondingly incremental ploughing thrust force. When examining forces for a worn tool in orthogonal cutting, the authors established two sets of forces, shearing and ploughing.

Each of the sets of forces was further divided into a cutting force and a thrust force. For ploughing, these were the ploughing cutting force, $F_{c u t}$, and the ploughing thrust force, $F_{p l}$. The force vital to the work in this dissertation was $F_{p l}$ and its application to drilling. 
The ploughing force model was analyzed and deemed appropriate for adaptation to drilling by combining the ploughing thrust force and the drilling thrust force. This established a new thrust force representing burr formation in day-to-day drilling conditions. Just like $F_{p l}, F_{t h}$ is based on orthogonal cutting. In combining the two forces, the thrust force for drilling remains the leading algorithm adapted to incorporate tool wear, and subsequently modified the burr height and thickness equations. A segmented drill bit cutting edge approach was also part of merging the two forces.

The new thrust force for drilling, $\Delta F_{T H-T O T A L}$, accounts for both ploughing and cutting mechanisms by introducing a wear/no wear condition via percentage of wear for each segment, thereby leading to a hybrid model for drilling burr formation. This reflects gradual wear of the drill bit cutting edge, which eventually leads to it being $100 \%$ worn and producing burrs, making it unfeasible to continue using the drill bit. The goal for this model was to come up with an indispensable tool for burr minimization that would balance the monitoring of burr growth and drill bit wear.

\subsection{Ploughing Force Model}

The ploughing mechanism in [15] is multifaceted. The ploughing force model functions within the primary plastic shear zone. It entails a worn tool cutting edge sliding and indenting the work, creating a build up, which is eventually sheared, instead of the standard chip formation and removal that is part of orthogonal cutting with a sharp tool.

The ploughing-force model is based on a slip-line model rooted in past research on slip-line field theories involving wedge sliding and chip cutting, for example, [39], [46], [47]. The recurring theme in these slip-line fields is a sliding wedge or a raised prow, moving a material forward, where the material in front of the wedge is squeezed and moved forward to join with the face of the prow. The work material then travels forward in a wave ahead of the wedge. The final slip-line field model for ploughing, utilized in this research to develop a novel drilling burr-formation model, is represented in Fig. 4.1. 


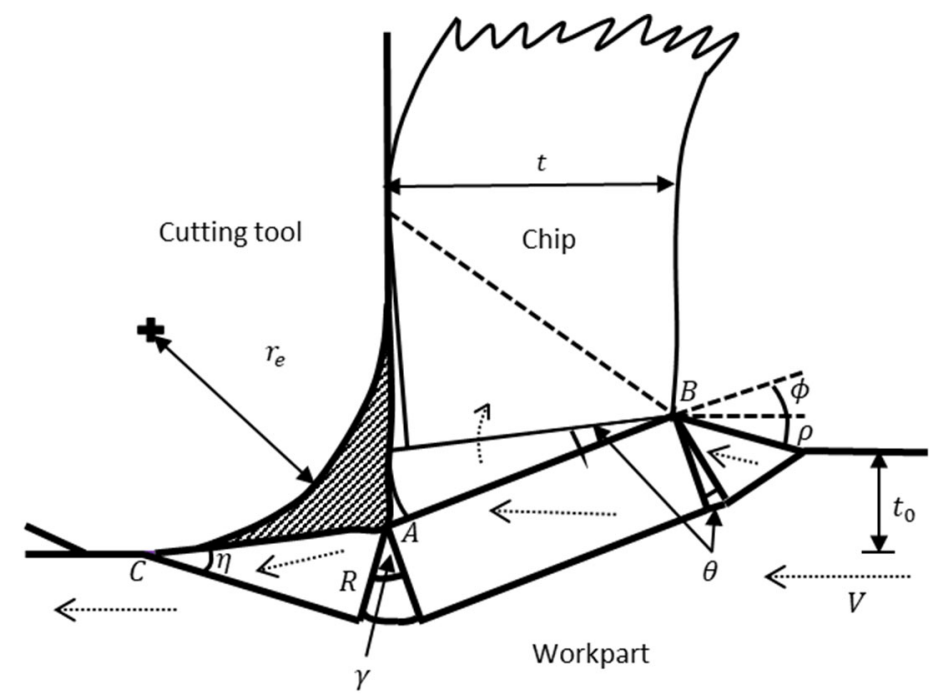

Fig. 4.1 Slip-line field model for ploughing in orthogonal cutting, adapted from [15, Fig. 3]

The model predicates that built-up work material is attached to the tool's finite cutting edge. Once a maximum build up of work material is reached, material flow is presumed to be directed at the boundary of the accumulated material, rather than splitting at the cutting edge.

The short dashed arrows in Fig. 4.1 pointing to the left illustrate the direction of material flow, in the direction of workpiece velocity $V$. This is directed equally into the chip and below the cutting tool, which has an edge radius $r_{e}$. At the break in the path of velocity on the lower part of the slip-line field, the workpiece is pushed up prior to the rounded tool cutting edge reaching it. The hatched section represents the stable material accumulation zone. Point $A$ is the point of stagnation at which material diverts. The segment $A B$ designates the type I slip line (equivalent to a conventional shear plane in orthogonal cutting), which is positioned as shear angle $\phi$. The cutting tool is situated with respect to the work at prow angle $\rho_{\text {row }} . t_{0}$ is the chip thickness prior to chip formation. Subsequent frictional and geometric relationships, detailed in [15], define the rest of the field below $\overline{A B}$, culminating in the ploughing thrust force, developed by [15], of most interest to this study, shown in eq. (4.1) [15, eq. (16)]:

$$
F_{p l}=k w[(1+2 \sin \theta+2 \gamma+\sin (2 \eta)) \cos (\phi-\gamma+\eta)-\cos (2 \eta) \sin (\phi-\gamma+\eta)] C A
$$

In eq. (4.1), the two key force parameters are $k$, the material shear strength, and $w$, the contact region width. The angles are angle projections, designated as fan field angles $\theta$ and $\gamma$. The angle between the slip line and the bottom surface of the built-up region is $\eta$. For detailed angle projection derivations see Appendix E, Section E.1. 
For the purposes of this dissertation, material shear flow $k$ is redefined to represent material shear strength. The details can be found in Appendix E, Section E.1, eqs. (E.4-E.6).

The next variable in eq. (4.1), $C A$, represents both the workpiece and the bottom surface of the material build-up region, as well as the zone where ploughing forces act. A component important to the new model developed in this chapter is $R_{\text {circ }}$, a subcomponent of $C A . R_{\text {circ }}$ is the radius of the circular fan field with its centre at $A$. For the purposes of the development of the ploughing force, the prow angle is set to approach zero, which simplifies $R_{\text {circ }} . r_{e}$, a component of $R_{\text {circ }}$, is the tool cutting edge radius. This will also be a critical parameter of the new tool wear model described in Section 4.3. For the details of $C A, R_{c i r c}$, and $r_{e}$, see Appendix E, eqs. (E.7-E.9).

\subsection{New Burr Formation Model: Combining Ploughing and Drilling Thrust Forces}

In this section, a new drilling thrust force is established, accounting for varying degrees of tool wear and incorporating elements of merged thrust forces, the drilling thrust force $F_{t h}$ and the ploughing force $F_{p l}$. The approach is novel in its combination of a drilling burr formation model and a slip-line field model for orthogonal cutting. The development of the new thrust force begins by looking at the drilling thrust force equation, eq. (A.12) [12, eq. (15)], discussed in Appendix A, Section A.1:

$$
F_{t h}=\frac{\sqrt{2}}{3} R f \cdot \sigma_{u} \cdot \sum_{i=1}^{N}\left\{\frac{\sin \left(\frac{\pi}{6}-\frac{\alpha_{d}}{2}\right)\left(\rho_{i+1-} \rho_{i}\right)}{\sin \left(\frac{\pi}{6}+\frac{\alpha_{d}}{4}\right) \cos \left(\frac{\pi}{3}-\frac{\alpha_{d}}{4}\right)}\right\}
$$

where eq. (3.13) [12, eq. (26)]:

$$
\sum_{i=1}^{N}\left\{\frac{\sin \left(\frac{\pi}{6}-\frac{\alpha_{d}}{2}\right)\left(\rho_{i+1-} \rho_{i}\right)}{\sin \left(\frac{\pi}{6}+\frac{\alpha_{d}}{4}\right) \cos \left(\frac{\pi}{3}-\frac{\alpha_{d}}{4}\right)}\right\}=\Phi
$$

Next, taking the ploughing thrust force, eq. (4.1) [15, eq. (16)]:

$$
F_{p l}=k w[(1+2 \sin \theta+2 \gamma+\sin (2 \eta)) \cos (\phi-\gamma+\eta)-\cos (2 \eta) \sin (\phi-\gamma+\eta)] C A
$$

The ploughing thrust force stems from orthogonal cutting, and since the new method is targeted for drilling, the ploughing thrust force must be transformed from orthogonal to oblique cutting. 
The modification began by adopting a segmented cutting edge of the drill, described in Section 3.2. The ploughing thrust force became $\Delta F_{p l}$ and was the thrust force acting on each segment:

$$
\Delta F_{p l}=k \Delta w[(1+2 \sin \theta+2 \gamma+\sin (2 \eta)) \cos (\phi-\gamma+\eta)-\cos (2 \eta) \sin (\phi-\gamma+\eta)] C A
$$

where $\Delta w$ is the length of the cutting edge segment being considered.

The next step in the modification involved incorporating $r_{i+1} r_{i}$ into the ploughing force, which identified the length of each consecutive segment on the segmented drill bit cutting edge. This variable comes from the relative radius in Appendix A, Section A.1, $\rho=r / R$, eq. (A.4), [12], where $r_{i}$ represents the distance of each segment on the sectioned drill bit cutting edge and $R$ is the drill bit radius. Therefore:

$$
\Delta w=\left(r_{i+1-} r_{i}\right)
$$

and

$$
[(1+2 \cdot \theta+2 \cdot \gamma+\sin (2 \cdot \eta)) \cdot \cos (\phi-\gamma+\eta)-\cos (2 \cdot \eta) \cdot \sin (\phi-\gamma+\eta)]=\text { angles }
$$

This set of equations is re-identified as "angles" for the sake of simplicity in subsequent force conversions. Re-writing the $\Delta F_{p l}$ relationship with the above substitutes:

$$
\Delta F_{p l}=k \cdot\left(r_{i+1-} r_{i}\right) \cdot[\text { angles }] \cdot C A
$$

The ploughing force conversion is continued through the incorporation of the dynamic rake angle $\alpha_{d}$, eq. (A.10) [12, eq. (12)]. The rake angle in $R_{c i r c}$ is replaced with the dynamic rake angle $\alpha_{d}$, as $\alpha_{d}$ will also be a function of each drill cutting edge segment under consideration and of $r_{i}$. $R_{\text {circ }}$. Therefore, it becomes $R_{\operatorname{circ}_{i}}$ :

$$
R_{\operatorname{circ}_{i}}=\sin (\eta) \times \sqrt{\left(r_{e} \cdot \tan \left(\frac{\pi}{4}+\frac{\alpha_{d}}{2}\right)\right)^{2}}
$$


Consequently, $C A$ will be subscripted with $i$ as it is also a function of $R_{\text {circ }}$ :

$$
C A_{i}=\frac{R_{\operatorname{cir} c_{i}}}{\sin (\eta)}
$$

Next, incorporating the sum to aggregate segments $N$ over the entire length of the cutting edge of the drill, just as in the drilling thrust force:

$$
F_{p l}=\sum_{i=1}^{N} k \cdot[\text { angles }] \cdot C A_{i} \cdot\left(r_{i+1-} r_{i}\right)
$$

Summing the elements across the cutting edge and incorporating components of $C A_{i}$, eq. (4.8) expands to:

$$
F_{p l}=\frac{\sigma_{y}}{\sqrt{3}} \cdot[\text { angles }] \sum_{i=1}^{N} \frac{R_{\text {circ }}}{\sin (\eta)} \cdot\left(r_{i+1-} r_{i}\right)
$$

Incorporating an expanded $R_{\operatorname{circ}_{i}}$ :

$$
F_{p l}=\frac{\sigma_{y}}{\sqrt{3}} \cdot[\text { angles }] \cdot \sum\left(r_{e} \cdot \tan \left(\frac{\pi}{4}+\frac{\alpha_{d}}{2}\right)\right) \cdot\left(r_{i+1-} r_{i}\right)
$$

Next, combining the drilling thrust force and the ploughing thrust force to form the basis of the new method to show the impact of tool wear on burr growth:

$$
F_{T H-T o t a l}=F_{t h}+F_{p l}
$$

Expanding the new combined thrust force that now incorporates all totals, including the sum of forces on all segments along the drill bit cutting edge:

$$
F_{T H-T o t a l}=\left\{\frac{\sqrt{2}}{3} R f \sigma_{u} \Phi\right\}+\left\{\frac{\sigma_{y}}{\sqrt{3}} \cdot[\text { angles }] \cdot \sum\left(r_{e} \cdot \tan \left(\frac{\pi}{4}+\frac{\alpha_{d}}{2}\right)\right) \cdot\left(r_{i+1-} r_{i}\right)\right\}
$$

For a flowchart recapping the formulation of $F_{T H-T o t a l}$, see Appendix $\mathrm{F}$. 
The new thrust force is further enhanced through the addition of percentage weights $\omega_{1}$ and $\omega_{2}$, which add up to $100 \%$. This results in demonstrating a \% sharp and \% dull tool, allowing this model to be reflective of realistic machining conditions:

$$
\omega_{1} F_{t h}+\omega_{2} F_{p l}=F_{T H-T o t a l}
$$

The $\%$ wear, and therefore the $\omega_{1}$ and $\omega_{2}$ weights, were assigned based on the level of wear of the drill bit cutting edge radius. With respect to the segmented cutting edge approximation, the wear radius was uniformly applied in the same manner to each individual segment across the entire drill cutting edge. The cutting edge radius, therefore, quantifies the percentage wear as the weights were assigned according to the level of wear of the drill bit radius. If the drill cutting edge radius is measured to be, for example, $25 \%$ worn $\left(\omega_{2}=0.25\right)$, then it is $75 \%$ sharp $\left(\omega_{1}=0.75\right)$. Each discrete segment in the approximation will have the weights applied in the same manner.

\subsection{Development of New Initial Chip Thickness}

The goal of establishing a new thrust force weighting model with a wear/no-wear condition was to utilize it to develop burr height and thickness growth models. As described in Section 3.4, this is calculated using the approximation of the initial chip thickness.

Revising $t_{0}$ requires incorporating the new $F_{T H-T o t a l}$ into $t_{0}$. To achieve this, the subcomponents of $t_{0}$ that solve it are revised by returning to the symbolic representations of the quadratic equation that solves $t_{0}$, beginning with $t_{0}$, eq. (3.9) [12, eq. (26)]:

$$
t_{0}=\frac{-J_{2}+\sqrt{J_{2}{ }^{2}-4 J_{1} J_{3}}}{2\left(J_{1}\right)}
$$

where

$$
\underbrace{(A C+D) t^{2}}_{J_{1}}+\underbrace{\left(A B-\frac{1}{3} F\right) t}_{J_{2}} t-\underbrace{\frac{1}{3} F \frac{R}{\tan p}}_{J_{3}}=0
$$


And as previously derived from [12, eq. (26)] :

$$
\begin{gathered}
(A C+D)=J_{1}=\frac{3}{4} \pi \sin p^{3} \cos p+\frac{1}{2} \pi\left(\frac{\pi}{2}-p\right) \\
\left(A B-\frac{1}{3} F\right)=J_{2}=\frac{3}{4} \pi R \sin p\left(\ln \left(\frac{1}{\sin p}\right)\right)-\frac{\sqrt{2}}{9} f \sigma_{u} \cdot \Phi \\
\left(\frac{1}{3} F \frac{R}{\tan p}\right)=J_{3}=\frac{\sqrt{2}}{9} f\left(\frac{\sigma_{u}}{\sigma_{y}}\right)\left(\frac{R}{\tan p}\right) \cdot \Phi \\
\Phi=\sum_{i=1}^{N}\left\{\frac{\sin \left(\frac{\pi}{6}-\frac{\alpha_{d}}{2}\right)\left(\rho_{i+1-} \rho_{i}\right)}{\sin \left(\frac{\pi}{6}+\frac{\alpha_{d}}{4}\right) \cos \left(\frac{\pi}{3}-\frac{\alpha_{d}}{4}\right)}\right\}
\end{gathered}
$$

The only subcomponents affected by incorporating the new thrust force will be those in eq. (3.8), which already contain the thrust force $\mathrm{F}$, meaning $J_{2}$ and $J_{3}$. To reflect the incorporation of $F_{T-T o t a l}$, all subcomponents are assigned a' for each variable. The redefined eq. (3.15) becomes:

$$
t_{0}^{\prime}=\frac{-J^{\prime}{ }_{2 \prime}+\sqrt{J^{\prime}{ }_{2}{ }^{2}-4 J^{\prime}{ }_{1}{ }^{\prime}{ }_{3}}}{2\left(J_{1}^{\prime}\right)}
$$

Further redefinition is as follows in eqs. (4.15-4.17). It is noted that $J_{1}{ }^{\prime}$ remains unchanged from $J_{1}$, as it does not contain the thrust force:

$$
\begin{aligned}
&\left(A^{\prime} C^{\prime}+D^{\prime}\right)=J_{1}{ }^{\prime}=\frac{3}{4} \pi \sin p^{3} \cos p+\frac{1}{2} \pi\left(\frac{\pi}{2}-p\right) \\
&\left(A^{\prime} B^{\prime}-\frac{1}{3} F^{\prime}\right)= J_{2}{ }^{\prime} \\
&=\left(\left(\frac{3}{4} \pi R^{2} \sigma_{y} \sin p\right)\left(\ln \left(\frac{1}{\sin p}\right)\right)\right) \\
&-\frac{1}{3}\left[\left(\frac{\sqrt{2}}{3} R f\left(\frac{\sigma_{u}}{\sigma_{y}}\right) \sigma_{y} \Phi\right)\right. \\
&\left.+\left\{\frac{\sigma_{y}}{\sqrt{3}} \cdot[\text { angles }] \cdot \sum \sqrt{\left(r_{e} \cdot \tan \left(\frac{\pi}{4}+\frac{\alpha_{d}}{2}\right)\right)^{2}} \cdot\left(r_{i+1-1} r_{i}\right)\right\}\right]
\end{aligned}
$$




$$
\begin{aligned}
\left(\frac{1}{3} F \frac{R}{\tan p}\right)=J_{3}{ }^{\prime} & \\
= & -\frac{1}{3}\left(\left\{\frac{\sqrt{2}}{3} R f\left(\frac{\sigma_{u}}{\sigma_{y}}\right) \sigma_{y} \Phi\right\}\right. \\
& \left.+\left\{\frac{\sigma_{y}}{\sqrt{3}} \cdot[\text { angles }] \cdot \sum \sqrt{\left(r_{e} \cdot \tan \left(\frac{\pi}{4}+\frac{\alpha_{d}}{2}\right)\right)^{2}} \cdot\left(r_{i+1}-r_{i}\right)\right\}\right)\left(\frac{R}{\tan p}\right)
\end{aligned}
$$

$H$ and $T$ are also redefined as $H^{\prime}$, eq. (4.18), and $T^{\prime}$, eq. (4.19), as $\mathrm{t}_{0}{ }^{\prime}$ enables the calculation of the burr height and thickness incorporating varying degrees of tool wear.

$$
\begin{gathered}
H^{\prime}=\mathrm{t}^{\prime} \sin p \exp \left\{\frac{\sqrt{3}}{2} \ln \left(\frac{100}{100-\% R \cdot A .}\right)\right\} \\
T^{\prime}=\mathrm{t}^{\prime}{ }_{0} \sin p \tan p
\end{gathered}
$$

\subsection{New Model Evaluation and Discussion}

The new drilling burr formation model was evaluated using simulations in Matlab R2017b.

The impact of two different aluminum alloys, five different levels of tool geometries (drill-bit wear), and three different feeds (.002 in/rev, $.004 \mathrm{in} / \mathrm{rev}$, and $.006 \mathrm{in} / \mathrm{rev}$ ) were examined, culminating in the burr height and thickness data given below. The five different drill bit cutting edge radii values, representing five levels of drill bit wear, are measurements of real-life worn drill bits used in experimental validation. The details of how the levels of drill-bit wear were defined are explained in Chapter 5, Section 5.1.4.

First, the total thrust force trend was examined; it increased progressively with increased wear as shown in Fig. 4.2 and Fig. 4.3. 


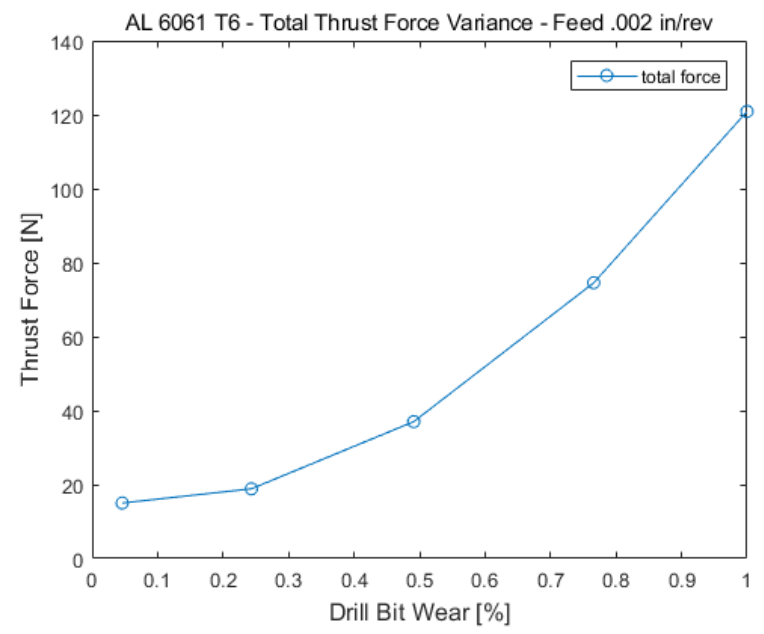

(a)

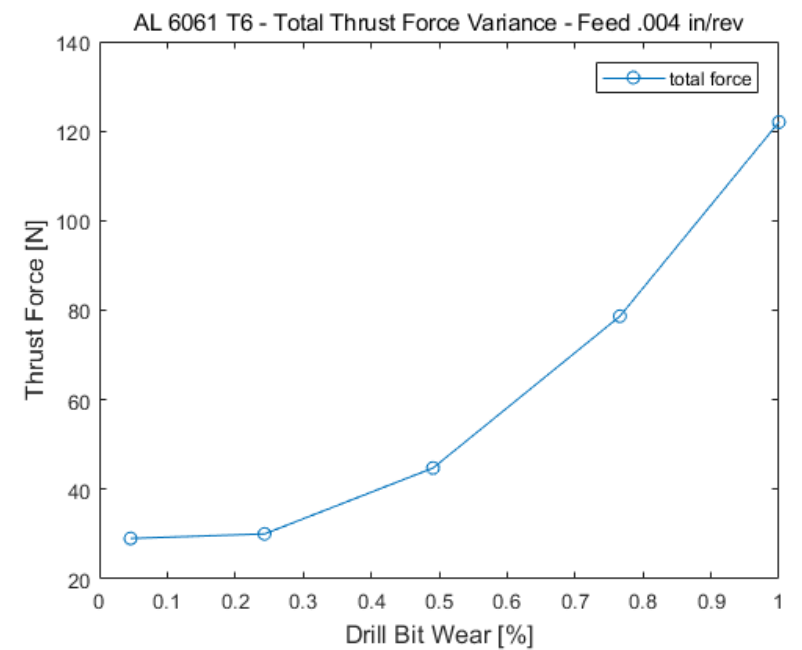

(b)

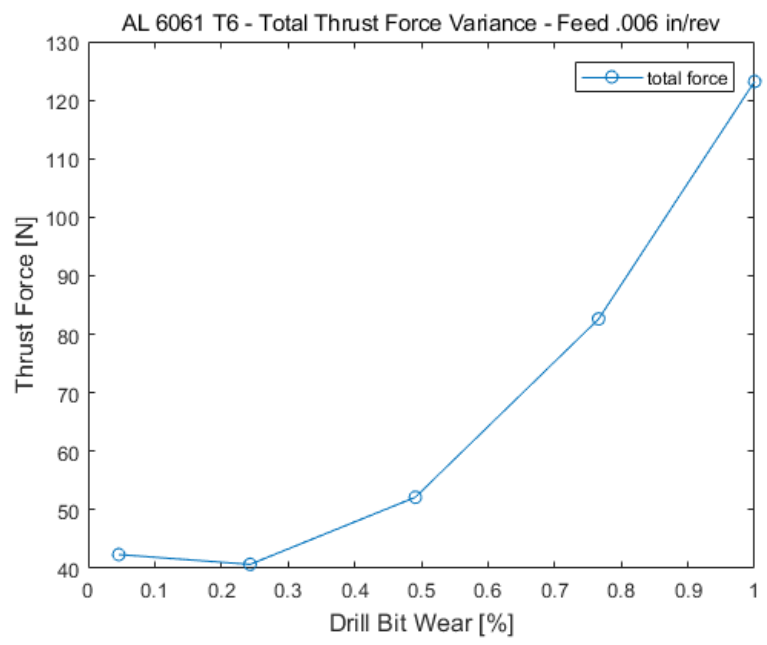

(c)

Fig. $4.2(\mathrm{a}-\mathrm{c})$ Calculated total drilling thrust force $[\mathrm{N}]$ in relation to drill bit wear [\%] for Al 6061 T6. The blue line with circles shows the calculated thrust force. 


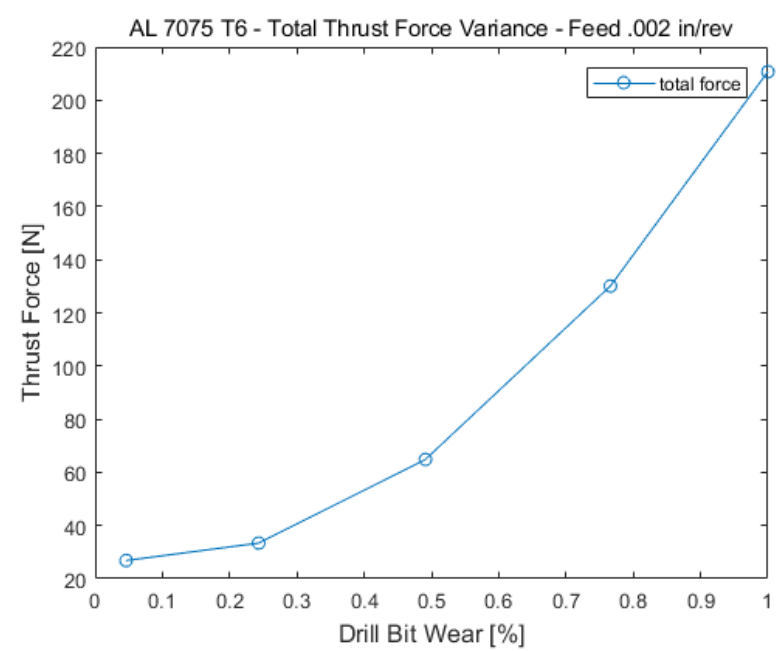

(a)

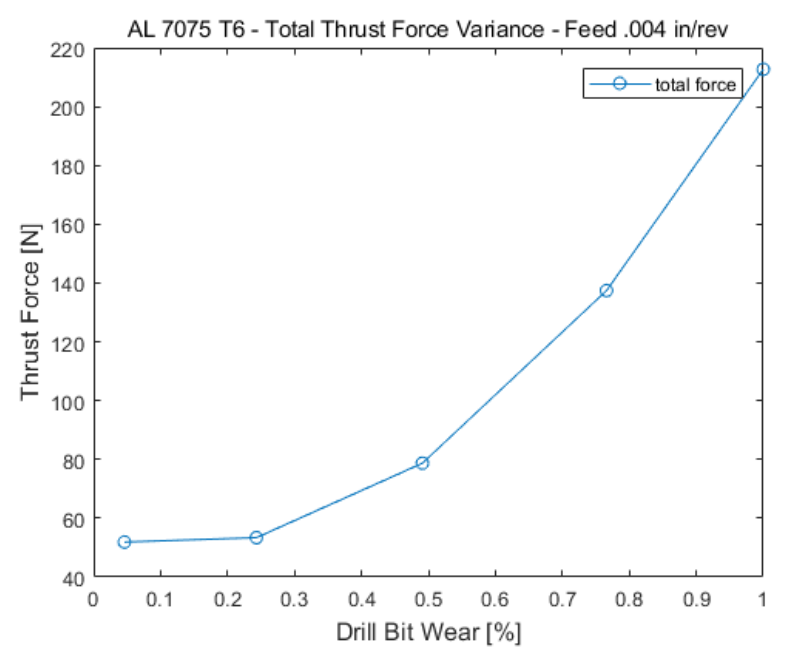

(b)

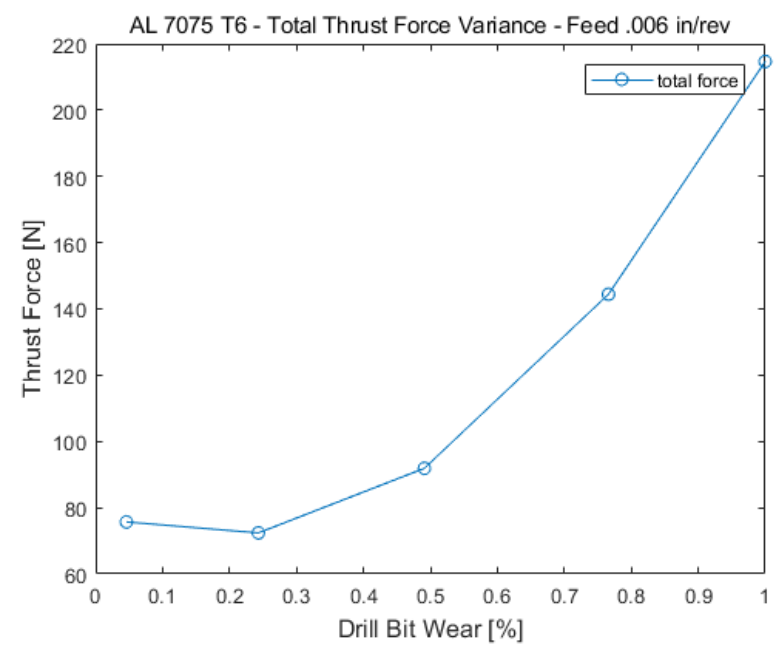

(c)

Fig. $4.3(\mathrm{a}-\mathrm{c})$ Calculated total drilling thrust force $[\mathrm{N}]$ in relation to drill bit wear [\%] for Al 7075 T6. The blue line with circles shows the calculated thrust force. 


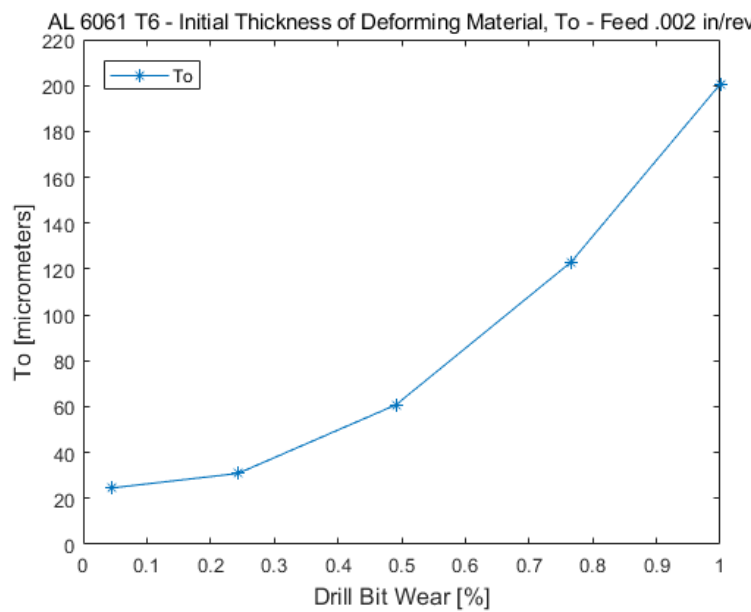

(a)

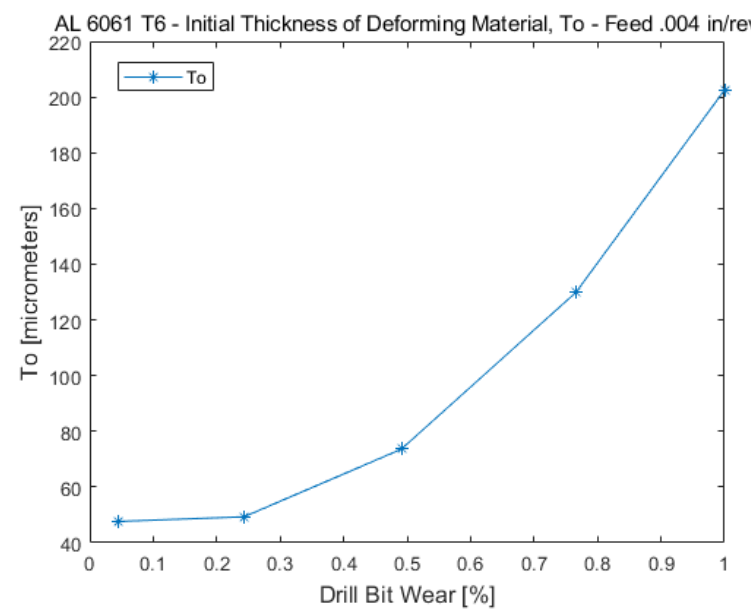

(b)

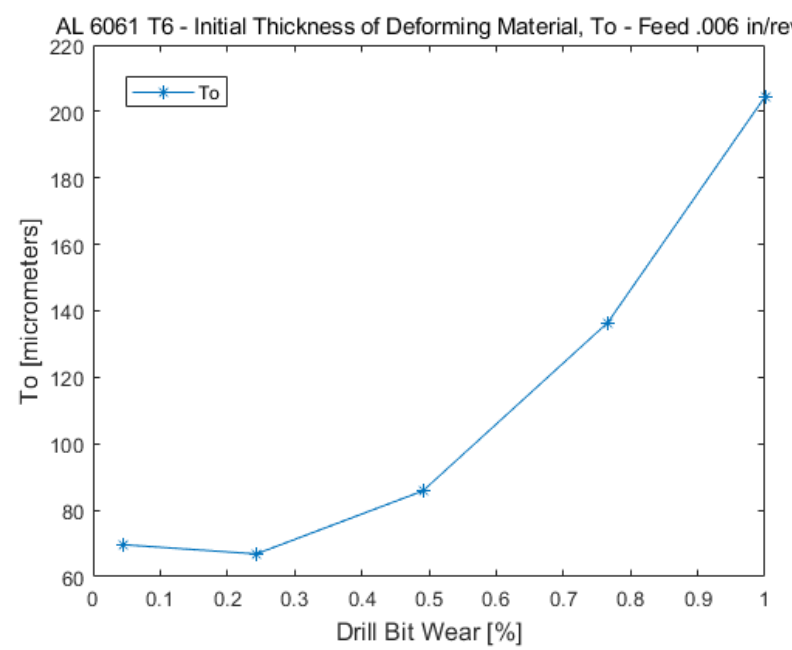

(c)

Fig. $4.4(\mathrm{a}-\mathrm{c})$ Calculated initial thickness of deforming material $t_{0}[\mu \mathrm{m}]$ in relation to drill bit wear [\%] for Al 6061 T6. The blue line with circles shows the calculated $t_{0}$. 


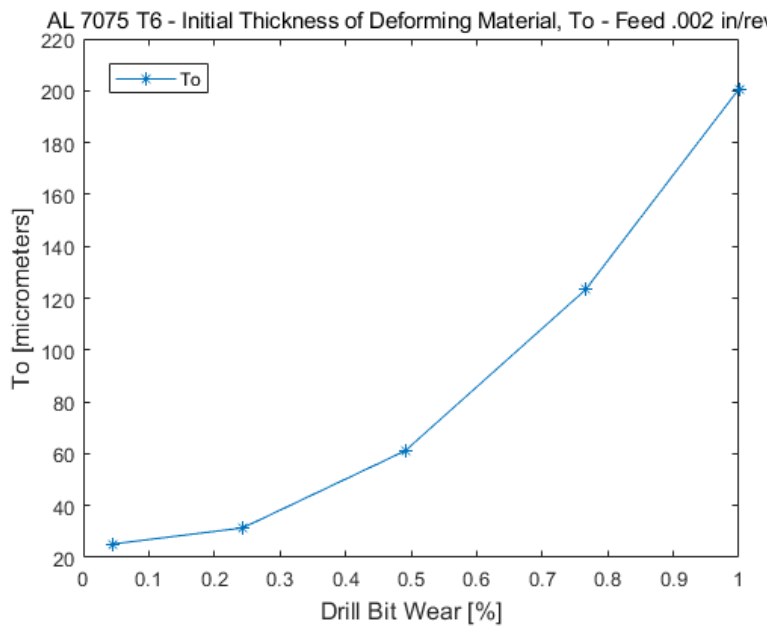

(a)

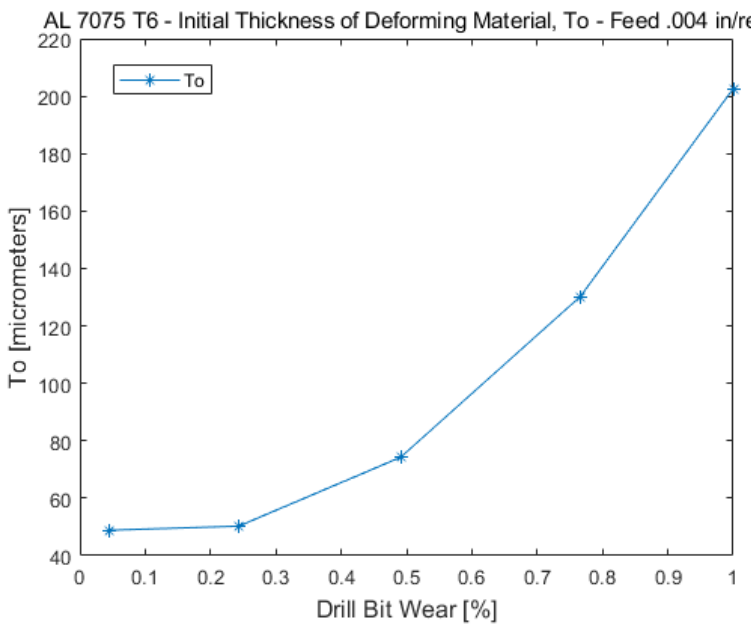

(b)

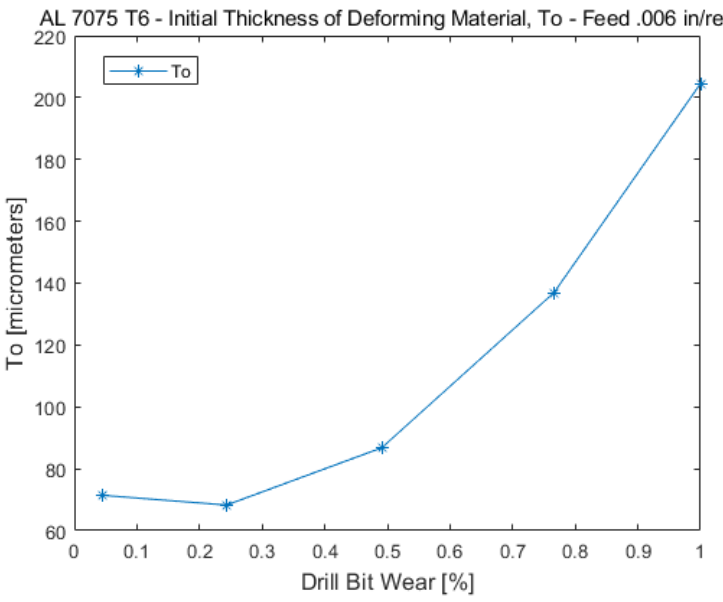

(c)

Fig. $4.5(\mathrm{a}-\mathrm{c})$ Calculated initial thickness of deforming material $t_{0}[\mu \mathrm{m}]$ in relation to drill bit wear [\%] for Al $7075 \mathrm{T6}$. The blue line with circles shows the calculated $t_{0}$. 
Correspondingly, the initial thickness of the deforming material, $t_{0}$, also increased as drill bit wear increased, as shown in the plots in Fig. 4.4 and Fig. 4.5. As described earlier in this chapter, it first became evident how burr height and thickness would behave from the trend of the initial thickness of the deforming material $t_{0}$.

The growth demonstrated in the results from these calculations is presented in the tables below. The established trend in the case of both materials is the same. As the level of wear increased, the resulting burr heights and thicknesses also increased.

Calculated burr heights and thicknesses at $0 \%$ drill bit wear ${ }^{2}$ drilled with an unused drill bit are values based on the initial burr formation model for sharp tools. This means a $0 \%$ weight is assigned to the ploughing force model and a $100 \%$ weight is assigned to the sharp thrust force model. In ideal drilling conditions, the ploughing force made zero contribution to the burr height and thickness calculation, because the drill bit cutting edge was sharp, as at $0 \%$ wear it had never been used before. On the other end of the wear spectrum, burr height and thickness values were computed from a $100 \%$ worn drill bit where calculations were performed using only the adapted ploughing force component.

For Al 6061 T6, the average growth between burr heights across three different feeds and a gradually increasing level of drill bit wear manifested as follows: less than $1 \%$ increase in burr height from $0 \%$ to $25 \%$ wear, $44 \%$ height increase from $25 \%$ to $50 \%$ drill bit wear, $69 \%$ height increase for wear growth from $50 \%$ to $75 \%$ drill bit wear and a $53 \%$ height increase from $75 \%$ to $100 \%$ drill bit wear. Burr thickness showed a very close burr growth pattern. For Al 7075 T6, the trend was comparable to that of burrs generated from Al 6061. The average difference between heights at a singular feed but different levels of tool wear was $3 \%$ for changes in burr height from $0 \%$ to $25 \%$ drill bit wear, $56 \%$ increase from $25 \%$ to $50 \%$ drill bit wear, $78 \%$ height increase for wear growth from $50 \%$ to $75 \%$ drill bit wear, and $56 \%$ height increase from $75 \%$ to $100 \%$ drill bit wear. The thickness for AL 7075 T6, just as for Al 6061 T6, demonstrated a very similar behaviour in terms of growth pattern.

\footnotetext{
${ }^{2}$ As established in Chapter 5, Section 5.1.4, the target drill bit wear levels were $0 \%, 25 \%, 50 \%, 75 \%$, and $100 \%$. In non-ideal conditions they were $4.61 \%, 24.31 \%, 49.1 \%, 76.6 \%$, and $100 \%$. For the purposes of this dissertation, moving forward, when discussing target $0 \%, 25 \%, 50 \%, 75 \%$, and $100 \%$ wear, the reference implies calculations using actual drill bit wear levels.
} 
Table 4.1 Al 6061 T6 simulation burr heights

\begin{tabular}{|c|c|c|c|c|}
\hline \multicolumn{2}{|c|}{ Drill Bit Characteristics } & \multicolumn{3}{c|}{ Al 6061 T6 - Burr height $[\mu \mathrm{m}]$ at three different } \\
feeds
\end{tabular}

Table 4.2 Al 6061 T6 simulation burr thicknesses

\begin{tabular}{|c|c|c|c|c|}
\hline \multicolumn{2}{|c|}{ Drill Bit Characteristics } & \multicolumn{3}{c|}{ Al 6061 T6 - Burr thickness $[\mu \mathrm{m}]$ at three } \\
different feeds
\end{tabular}

Table 4.3 Al 7075 T6 simulation burr heights

\begin{tabular}{|c|c|c|c|c|}
\hline \multicolumn{2}{|c|}{ Drill Bit Characteristics } & \multicolumn{3}{|c|}{ Al 7075 T6 - Burr height $[\mu \mathrm{m}]$ at three different } \\
feeds \\
\hline Drill bit wear & Drill Bit Radius & $.002 \mathrm{in} / \mathrm{rev}$ & $.004 \mathrm{in} / \mathrm{rev}$ & $.006 \mathrm{in} / \mathrm{rev}$ \\
\hline $0 \%$ & 0.01 & 28.4 & 55 & 80.4 \\
\hline $25 \%$ & 0.053 & 35.3 & 56.6 & 76.8 \\
\hline $50 \%$ & 0.107 & 68.8 & 83.6 & 97.7 \\
\hline $75 \%$ & 0.167 & 138.7 & 146.6 & 154.2 \\
\hline $100 \%$ & 0.218 & 225.9 & 228.1 & 230.2 \\
\hline
\end{tabular}


Table 4.4 Al 7075 T6 simulation burr thicknesses

\begin{tabular}{|c|c|c|c|c|}
\hline \multicolumn{2}{|c|}{ Drill Bit Characteristics } & \multicolumn{3}{|c|}{ Al 7075 T6 - Burr thickness $[\mu \mathrm{m}]$ at three } \\
different feeds
\end{tabular}

\subsection{Summary and Next Steps}

Once the two thrust-force models were combined and evaluated in the simulation, burr height and thickness increased gradually as was initially expected. There were no surges or sharp decreases in either burr height or thickness values. The steady and substantial climb in burr height and thickness values at each level of wear assured the validity of the novel method.

The combined thrust-force model resulted in an effective method of theoretically evaluating the impact of tool wear on drilling burr growth. The success of this combination stems from several aspects. Both Dornfeld's and Waldorf's models are based on orthogonal cutting. The sharp tool burr model was adapted to drilling. The ploughing force model was established for orthogonal cutting applications only, but its ploughing component had a thrust force element. As tool wear, and therefore ploughing, were of key interest to this research, the force format facilitated adjustment to drilling. Each model's focus on thrust force development enabled a successful merging of the two. The ploughing thrust force was successfully incorporated into the drilling thrust force to conceive an innovative approach to tool wear incorporation and in that way, a more realistic reflection of drilling conditions. The new burr-formation model will, therefore, help explain the burr growth variance with respect to the effects of tool wear. The model warranted further validation by experimentation, as detailed in Chapter 5. 


\section{CHAPTER 5 EXPERIMENTAL VALIDATION}

In order to evaluate the new burr-formation model, an experiment was conducted that reflected similar drilling conditions to those likely to be encountered by a machinist working with drill bits worn to different degrees. A series of drilling tests was executed, reflecting such conditions.

\subsection{Experimental Setup}

\subsubsection{Material Specifications}

The materials used were 6" × 6" sheets of aluminum alloys 7075 T6 and 6061 T6, at two material thicknesses, .040" and .090". Both alloys are in the group of aluminum alloys proven to have excellent machinability and therefore consistently deliver a good finish [48] - [50]. The two thicknesses are also commonly used in aerospace applications.

AL 6061 T6 has silicon and magnesium as the main alloying elements. Magnesium silicone delivers good weldability, formability, and corrosion resistance together with medium strength. It is more difficult to machine because of how magnesium and silicon are incorporated together [29]. AL $6061 \mathrm{~T} 6$ is one of the most adaptable from the heat treatable alloy group. AL 7075 T6 is commonly used for airframe structural components. Zinc is the chief alloying element, but the alloy may also contain levels of copper, magnesium, chromium, and zirconium. When a small proportion of magnesium is added, the 7XXX alloy group leads to heat-treatable alloys of very high strength.

The goal was to evaluate the burr formation behaviour of both materials and both thicknesses. The drilling parameters are detailed in subsequent sections.

Table 5.1 Material specifications

\begin{tabular}{|c|c|c|}
\hline Material & Yield Strength (MPa) & Tensile Strength (MPa) \\
\hline AL 6061 T6 & 241.31 & 324.05 \\
\hline AL 7075 T6 & 503 & 572.26 \\
\hline
\end{tabular}

\subsubsection{Drilling Parameters}

Drilling was performed using a CNC, HAAS Tool Room Mill Model TM1P, programmed with Mastercam, version X7. Drill bits utilized were from Clarkson-Osborn. 
The recommended cutting speed range for drilling aluminum alloys with high speed steel (HSS) drill bits per the Clarkson-Osborn tool catalogue is 95-250 surface feet per minute (SFM) [51]. ${ }^{3}$ For this experiment the cutting speed used for all operations was 100 SFM.

The recommended feed range for drilling aluminum alloys with HSS drill bits, diameter $1 / 8^{\prime \prime}$, is .002-.005 in/rev. The drill bit manufacturer notes that range is a general number and not a strict rule. For the purposes of this experiment, three different feeds were used in drilling, 0.002, 0.004, and $0.006 \mathrm{inc} / \mathrm{rev}$, to enable the examination of the impact of feed variation as a consequential parameter in burr formation, as described in Chapter 2. The upper feed range was increased to $.006 \mathrm{in} / \mathrm{rev}$ to observe burr behaviour at a greater feed.

Drill bits used were Clarkson Osborn HSS Bright Finish Jobber Length Drill, $0.1285^{\prime \prime}$ dia., $135^{\circ}$ point angle (\#30 drill bit) in order to generate $0.125 "$ dia. holes. This hole size is very commonly used in aerospace manufacturing for rivet installation. The goal of this experiment was to evaluate the new burr formation model but also to establish a practical link to a possible future application of the model in the aerospace manufacturing industry.

The aluminum sheets were drilled in a $4 \times 8$ hole pattern of four rows and eight columns as shown in Fig. 5.1. Each sheet was drilled with an individual drill bit worn down to a specified level. Each of the four rows was drilled with a separate feed value: row $1-.002 \mathrm{in} / \mathrm{rev}$, rows 2 and $3-.004 \mathrm{in} / \mathrm{rev}$, and row $4-$ $.006 \mathrm{in} / \mathrm{rev}$. A drilling jig was manufactured for the experiment on which the sheet metal was mounted, as can be seen in Fig. 5.2. Oversized holes were pre-drilled into the jig in the pattern shown in Fig. 5.1 as a relief area. An initial test run was conducted with the jig without the oversized holes, but it gave a false result because the jig supported the sheet metal and suppressed the burr formation.

\footnotetext{
${ }^{3} \mathrm{SFM}$ is the number of linear feet that a location on a rotating component travels in one minute, most commonly used in the measurement of cutting speed (surface speed) in machining. It is a unit of velocity describing how fast the cutting edge of the cutting tool is traveling. SFM is a combination of diameter and velocity (revolutions per minute) of the material measured in feet-per-minute as the spindle of a milling machine or lathe. SFM is calculated as follows: $R P M($ spindle speed $)=\frac{12 \times S F M}{\pi \times \text { drill bit diatmeter }(\text { in })} ; S F M=\operatorname{drill~bit~diameter~}($ in $) \times \pi \times \frac{1(f t)}{12(\text { in })} \times R P M$
} 


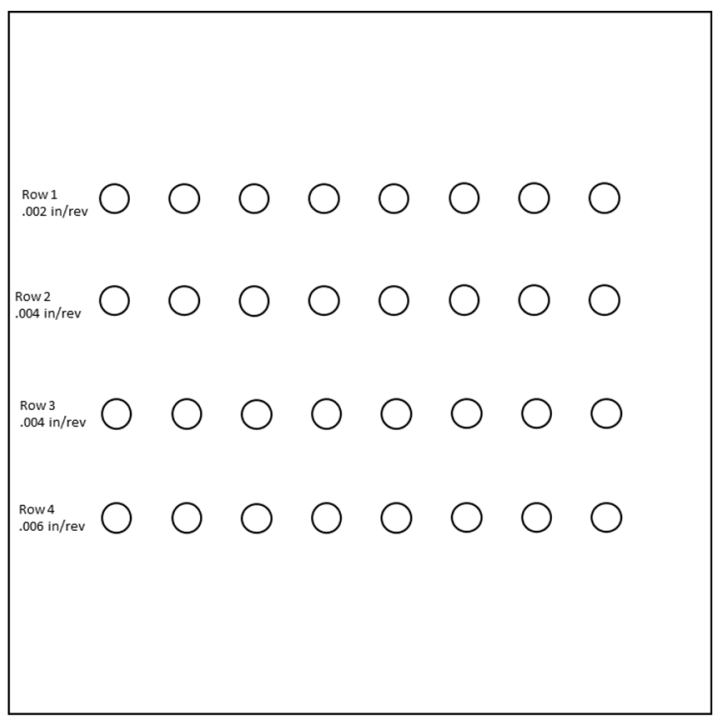

Fig. 5.1 Drilling test article drawing

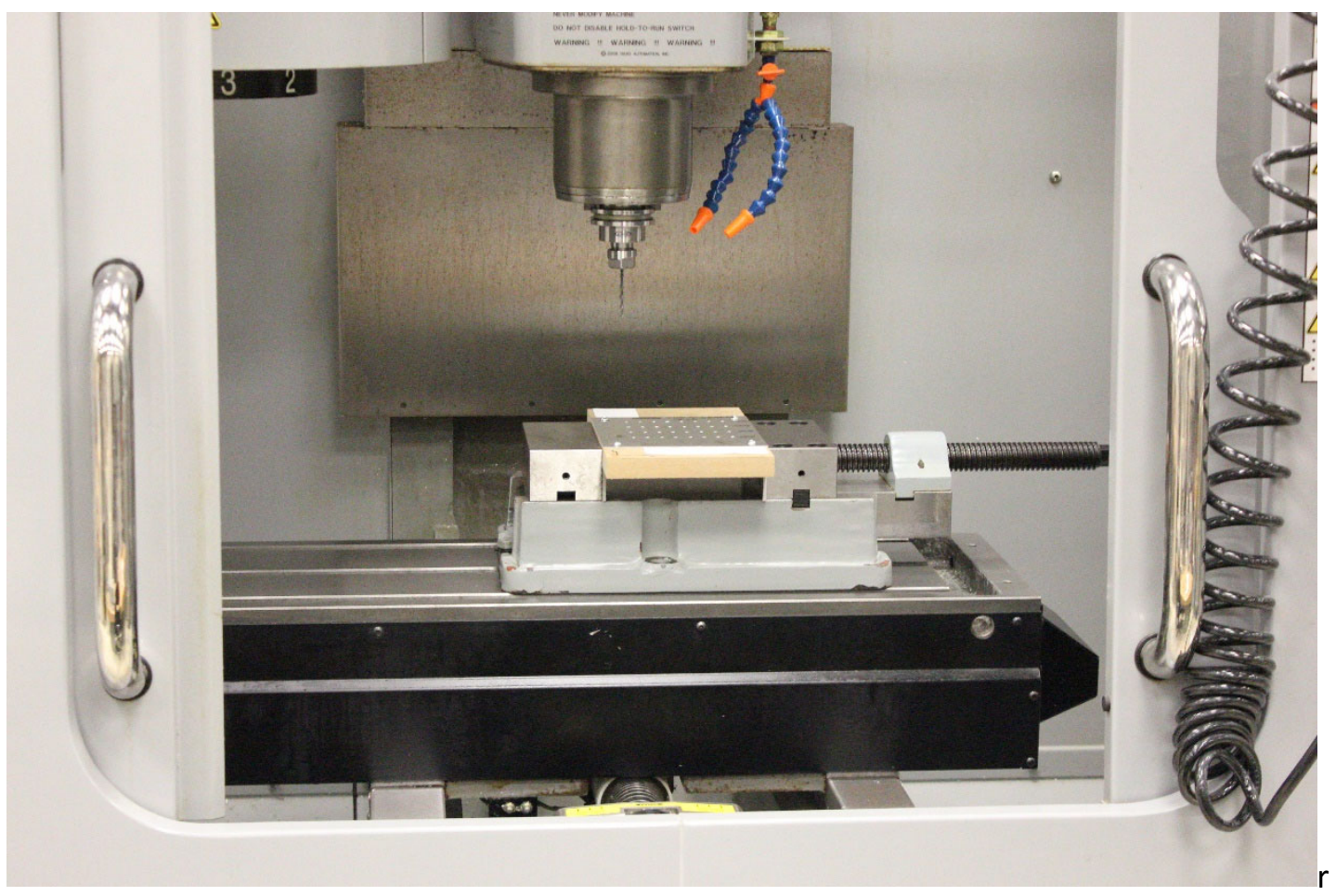

Fig. 5.2 CNC machine setup for drilling

\subsubsection{Drill Bit Preparation}

Five drill bits were used to demonstrate the relationship between drill bit wear and burr growth. Four of the drill bits had their cutting edges worn down to various degrees and one drill bit remained sharp. The 
drill bits were worn down artificially using a standard Dremel tool by Mastercraft with a flat grinding disk. This was done in order to accelerate testing. Simulated wear or damage to test articles is a common practice, enabling the obtaining of results that would otherwise take a long time to observe during normal wear and tear.

A relevant example is in aircraft damage tolerance and fatigue evaluation testing of principal structural elements [52]. Moreover, it is a Federal Aviation Administration requirement to produce simulated cracks on an aircraft structure being examined that portray fatigue damage of the element. This is done to ensure that should fatigue, corrosion, or accidental damage occur to the aircraft, the undamaged structure can endure expected loads in flight and on the ground until the damage is found during maintenance checks. Considering these applied examples, it is important to accelerate testing to obtain timely results.

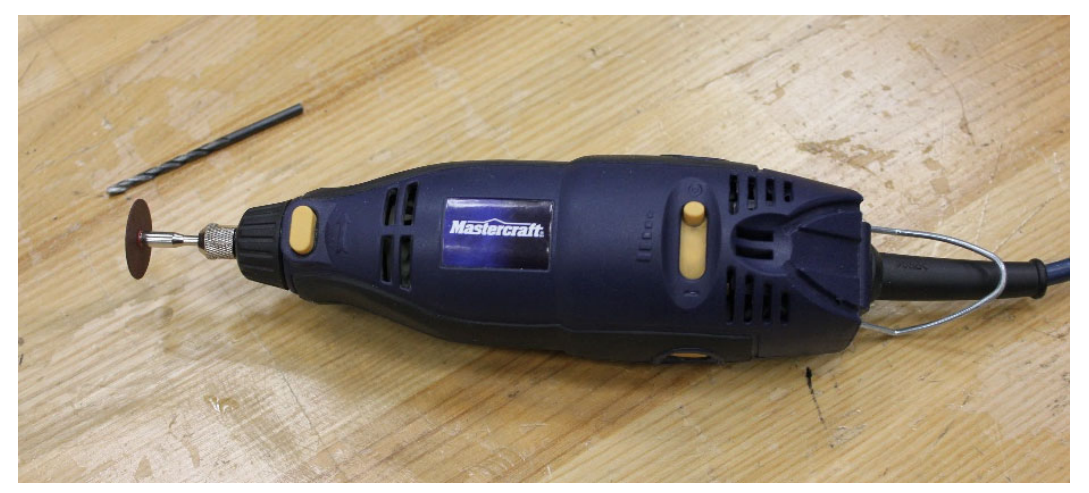

Fig. 5.3 Dremel tool with flat grinding disk used for drill bit wear down

The degree of artificial wear was varied with respect to the number of strokes made by the Dremel tool. Once worn down, each drill bit cutting edge was viewed with a JEOL scanning electron microscope (SEM) in Ryerson University's KHE 32 Lab, model JSM-6380LV, with a resolution of $3.0 \mathrm{~nm}$. 

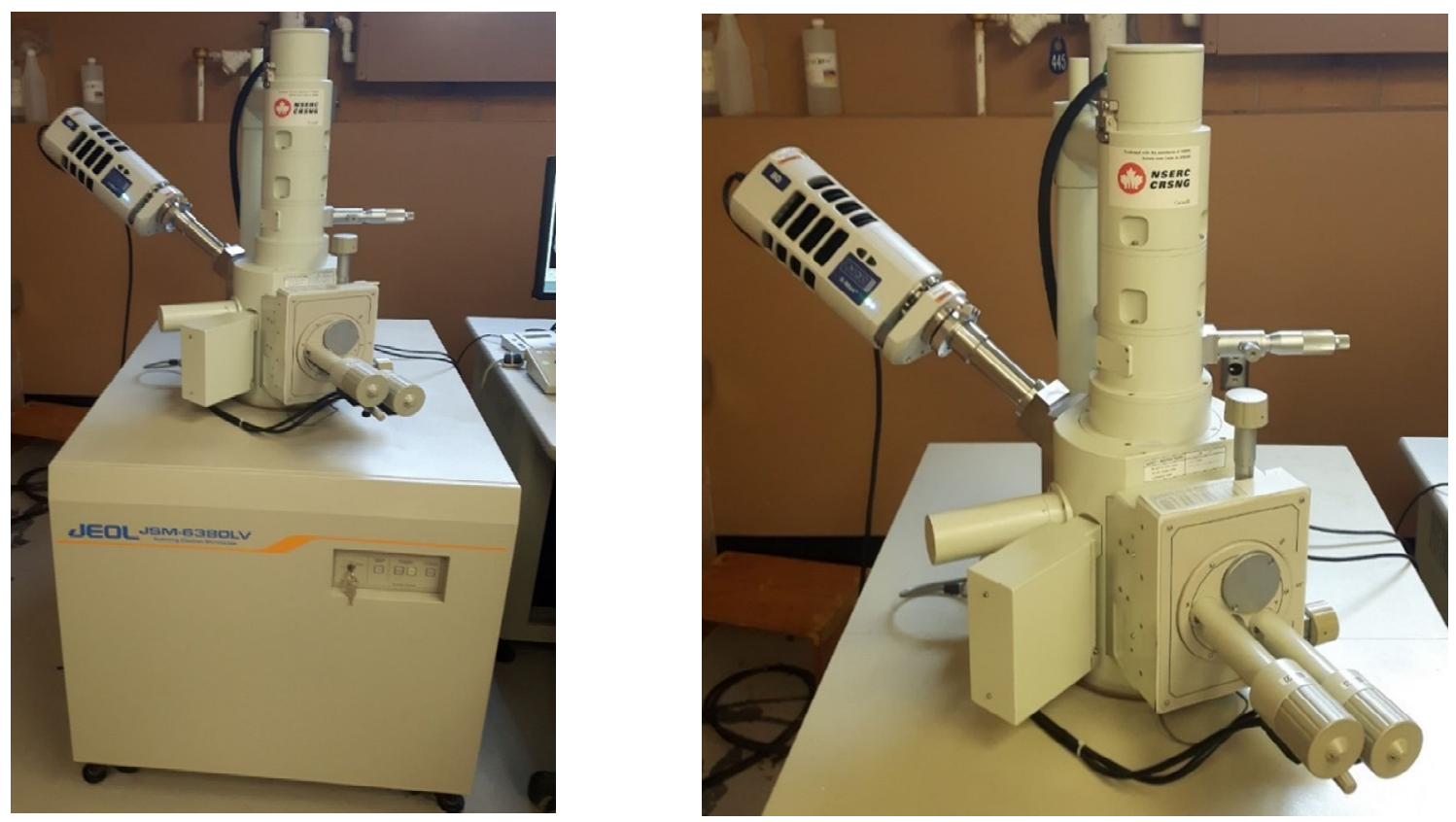

Fig. 5.4 Scanning electron microscope

The SEM picture of a drill bit worn to $25 \%$ wear can be seen in Fig. 5.5.

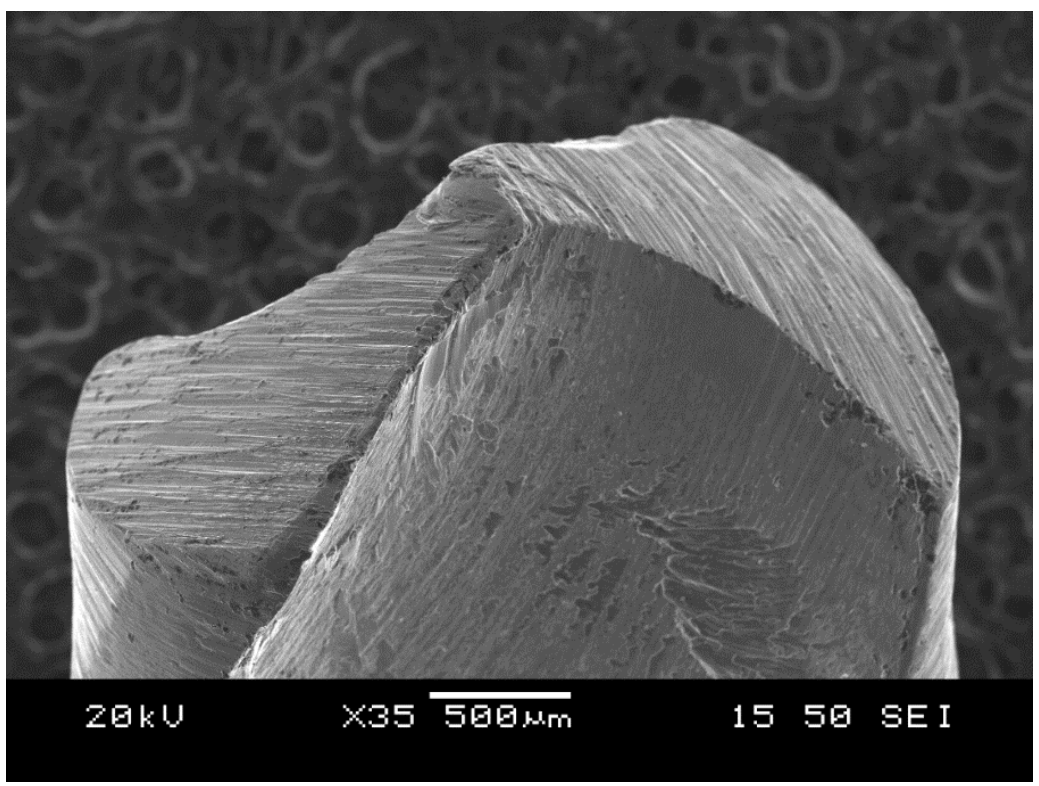

Fig. $5.525 \%$ worn drill bit, $35 x$ magnification 
The SEM photographs were analyzed by 3D imaging software, MeX Metrology by Alicona, where a 3D measurable image was created. The image of a $25 \%$ worn drill bit cutting edge is shown below in Fig. 5.6.

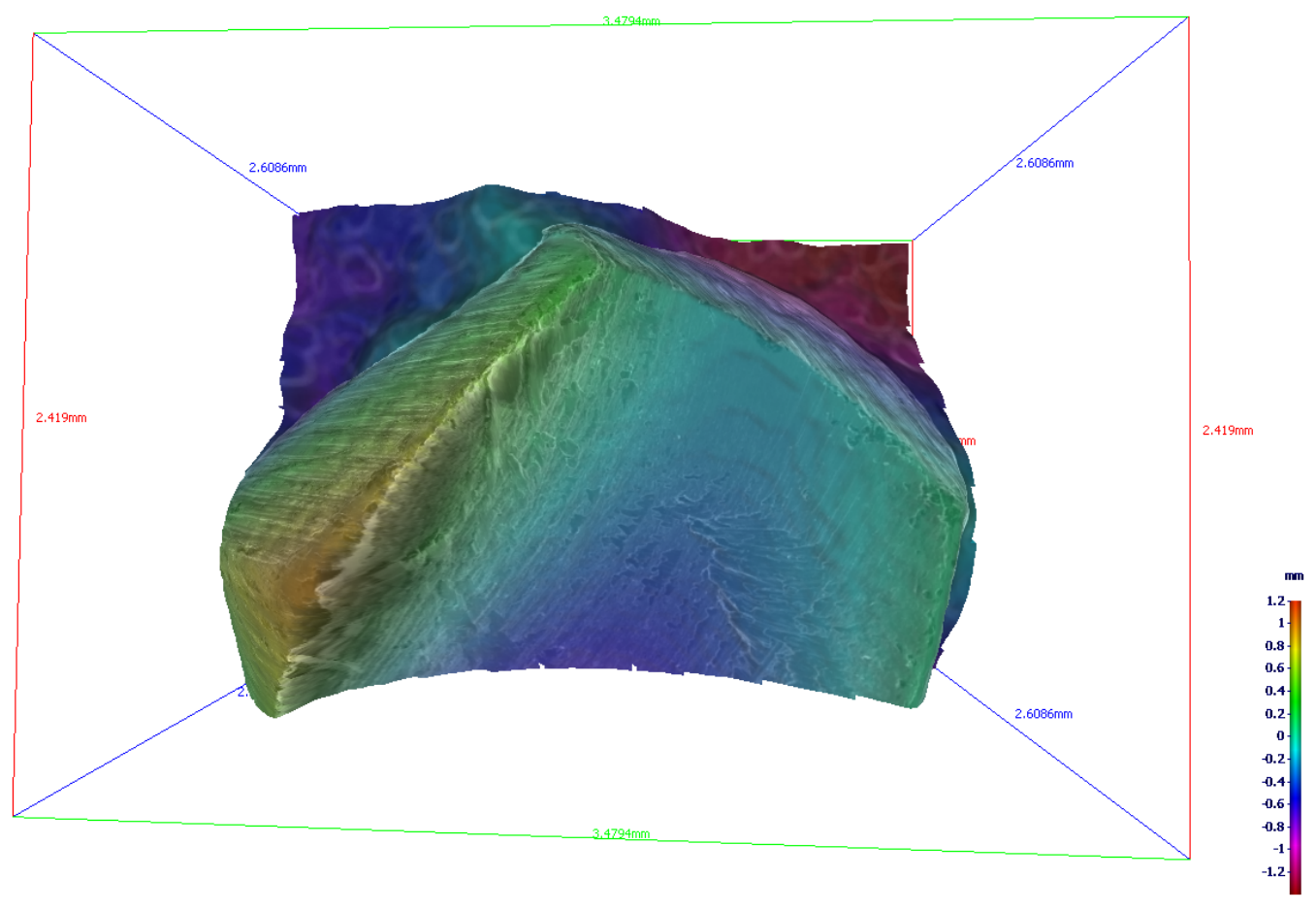

Fig. $5.63 \mathrm{D}$ image of $25 \%$-worn drill bits

The 3D image of each drill bit cutting edge enabled the selection of multiple edge sections, as shown by the red line segment, which is selecting a section, the slice of the drill cutting edge 3D data, in Fig. 5.7. These slices were then projected to show a 2D diagram of a slice of cutting edge as per Fig. 5.8. A circle was then superimposed onto the cutting edge and its radius measured with IC Measure, software from The Imaging Source Europe $\mathrm{GmbH}$, which enabled manual on-screen image measurement. The radius of the circle projected onto the cutting edge image became the measurement of the drilling cutting edge radius. Three measurements of each drill cutting edge were made for each of the five drill bits approximately $500 \mu \mathrm{m}$ from the outer cutting corner of the cutting edge of the drill bit. The average drill cutting edge radius value was calculated. 


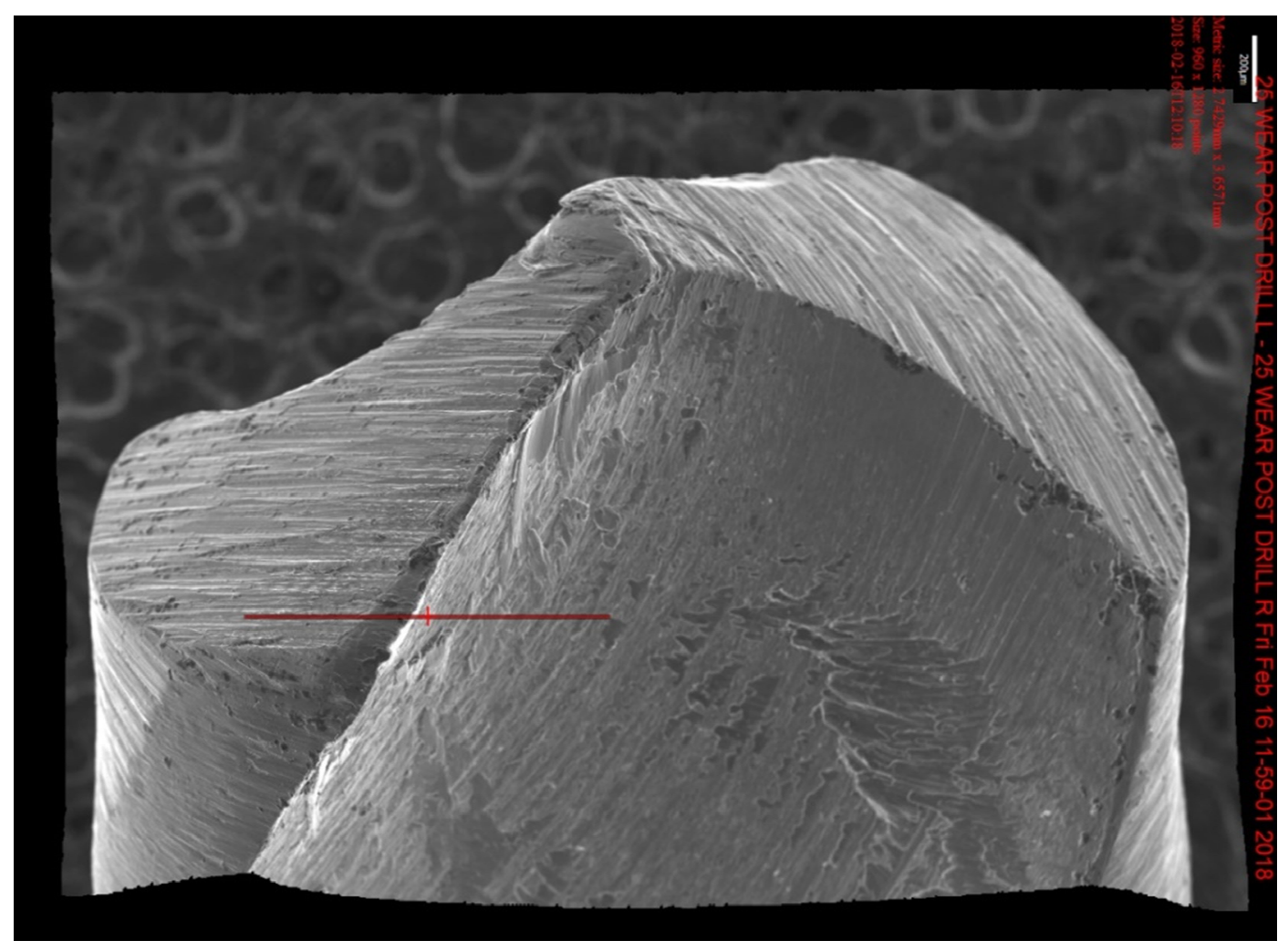

Fig. $5.725 \%$ worn drill bit, cutting edge slice selection

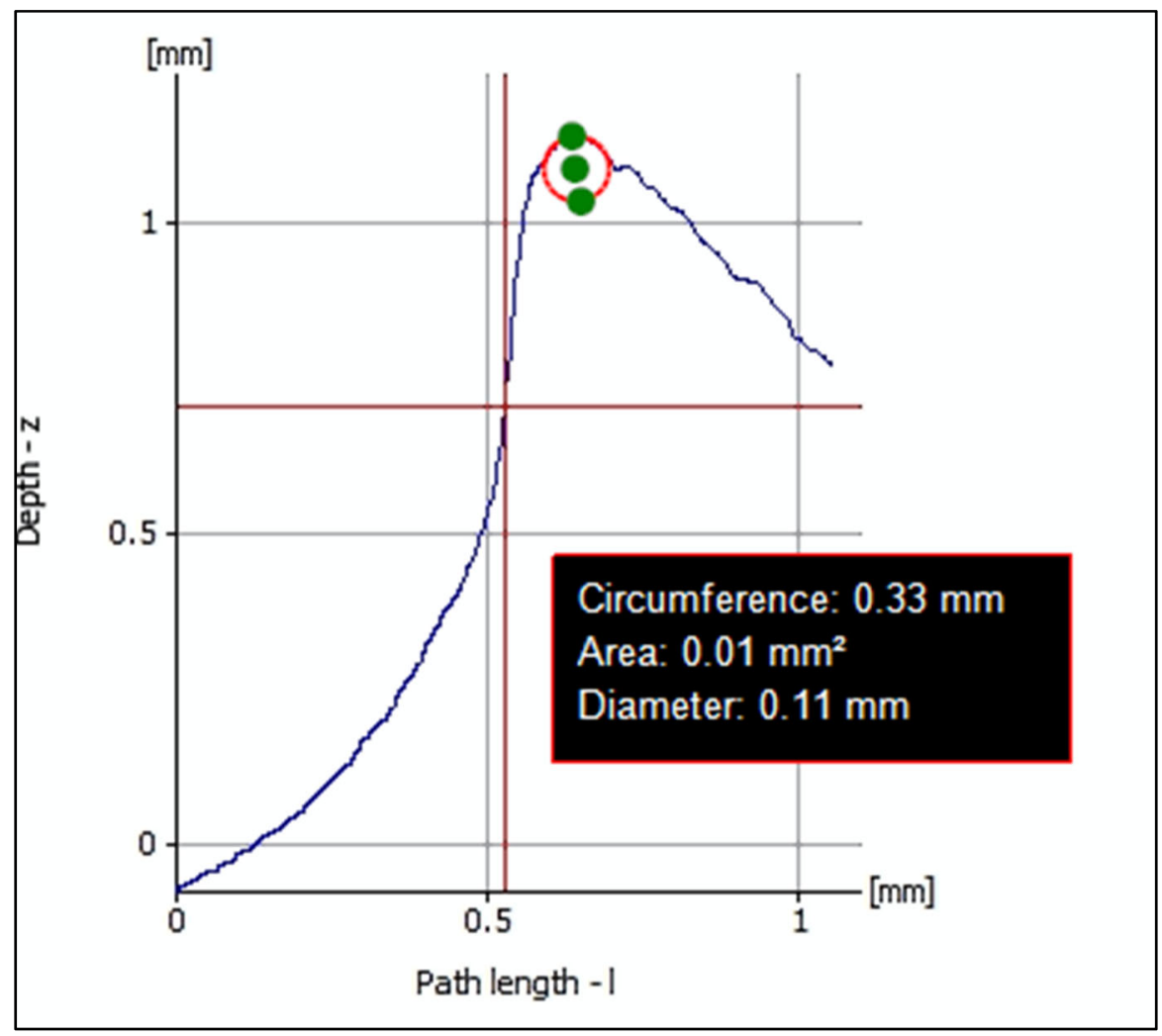

Fig. $5.825 \%$ worn drill bit, edge radius measurement 


\subsubsection{Establishing Levels of Drill Bit Wear}

In order to establish the range of worn drill bits at specific percentages of $0 \%, 25 \%, 50 \%, 75 \%$, and $100 \%$, a baseline level of wear was established at $100 \%$ wear, representing a completely worn drill bit. A tool is removed from service once it can no longer manufacture a satisfactory part per drawing [26]. Consequently, a drill bit that no longer generates a hole within the required drawing tolerance is taken out of use.

Drill bits were worn down manually with the Dremel tool to different wear levels. The drill bit cutting edge was then visually assessed with a magnifying glass, and subsequently measured with the SEM. Drill tests were performed with the worn down drill bits on sheets of AL 6061 T6 and 7075 T6 .040" and .090" thick. When a drill bit was unable to completely penetrate any of these materials, it was assigned the value of $100 \%$ wear, and its drill bit cutting edge radius measurement was determined to be $100 \%$ worn.

The drill bit cutting edge radius, $r_{e}$, for a drill bit that could not penetrate the material was measured in the SEM to be $.218 \mathrm{~mm}$. This process began with a target of achieving the following $\%$ of wear for each drill bit used: $0 \%, 25 \%, 50 \%, 75 \%, 100 \%$. Based on the maximum drill bit wear of $0.218 \mathrm{~mm}$, a linear relationship between radius and percent wear was established, enabling the calculation of the actual drill bit wear, as shown in eq. (5.1). The final results came close to the target, as listed in Table 5.2.

$$
r_{e}=.218(\% \text { wear })
$$

A brand new drill bit was measured to have a 0.01-mm cutting edge radius. For the purposes of this dissertation, whenever data is discussed in terms of target drill bit wear levels of $0 \%, 25 \%, 50 \%, 75 \%$, and $100 \%$, the reference is made to real-life drill bit wear conditions achieved.

Table 5.2 Drill bit edge radii measurements and wear calculation

\begin{tabular}{|c|c|c|}
\hline Target drill bit wear [\%] & Actual drill bit wear [\%] & $\begin{array}{c}\text { Measured drill bit } \\
\text { cutting edge radius } \boldsymbol{r}_{\boldsymbol{e}} \\
\text { [mm] }\end{array}$ \\
\hline 0 & 4.60 & 0.010 \\
\hline 25 & 24.31 & 0.053 \\
\hline 50 & 49.10 & 0.107 \\
\hline 75 & 76.60 & 0.167 \\
\hline 100 & 100.00 & 0.218 \\
\hline
\end{tabular}




\subsubsection{Observations and Drilling Test Results}

The experimental setup of .125"-diameter drilled holes and the produced burrs, an average all measuring less than $2 \mathrm{~mm}$ in height, led to the scrutiny and selection of the most appropriate method for burr measurement.

\subsubsection{Burr Measurement}

Burrs are typically defined by the burr descriptions and measurement categories shown in Chapter 3 , Fig. 3.6 [12, Fig. 7]:

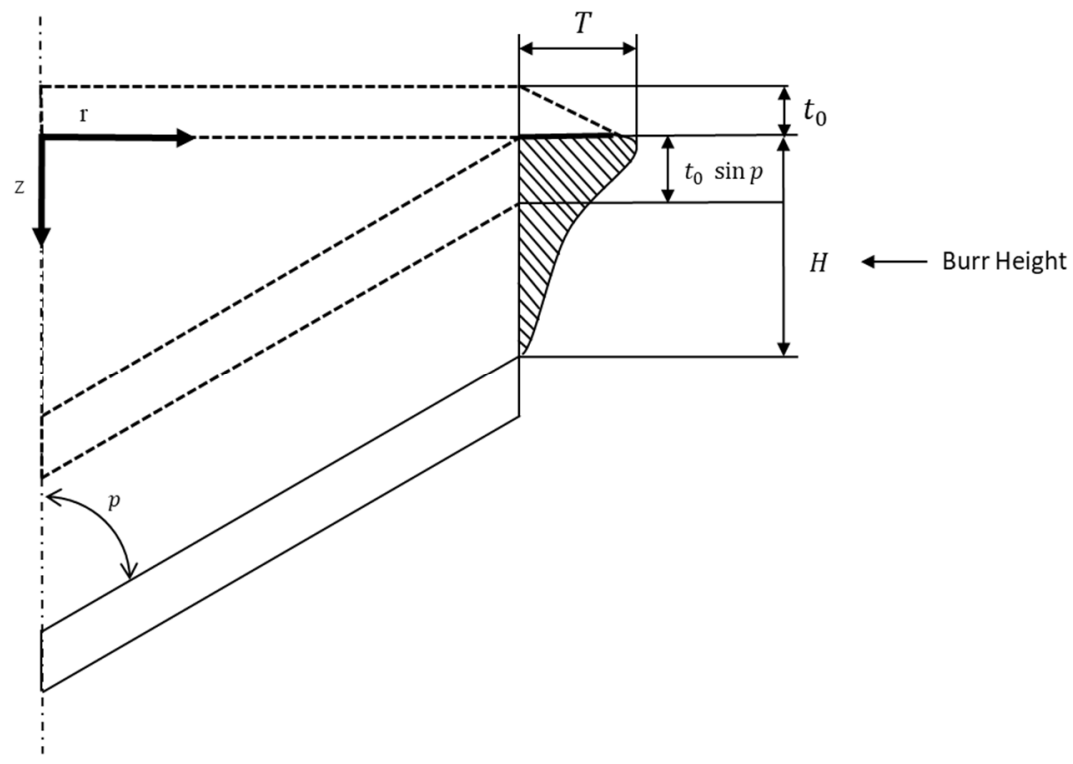

Fig. 3.6 Burr height and thickness formation, adapted from [12, Fig. 7]

As described in Chapter 3, Kim and Dornfeld [12] established the burr height and thickness formation model, as portrayed in Fig. 3.6. By estimating the initial thickness of deforming material, $t_{0}$, burr height and thickness were calculated through evaluating strain distribution and volume conservation of plastic deformation of work material around the circumference of the drilled hole. Kim and Dornfeld's burr height and thickness-graphical model overlaps to a degree with that of Schäfer [53], [54]. Schäfer used a burr cross section to describe basic burr characteristics, where burr height was determined by the span from the edge of the workpiece to the burr's highest point in the cross section. Burr thickness is the distance parallel to the burr root area, at a distance of $r_{f}$. Burr measurement with respect to the root versus the tip has relevance within experimental results as is subsequently described. 


\subsubsection{Burr Measurement Method}

There is a wide variety of burr measurement methods available. The choice of a given system depends on application conditions, requested measuring accuracy, and burr values to be measured such as burr height, thickness, volume, and hardness.

The range of current burr measurement and detection methods available, as described by Franke, Leitz, and Aurich [54], was evaluated and for the purposes of this experiment. The SEM was deemed the most appropriate because it afforded the necessary levels of magnification and accuracy, and measuring software. The software that was part of the SEM enabled live digital measurement of burr height and thickness and the capturing of a digital image of the burr, like the one show in Fig. 5.9, with the ability to superimpose height and thickness measurements on the image.

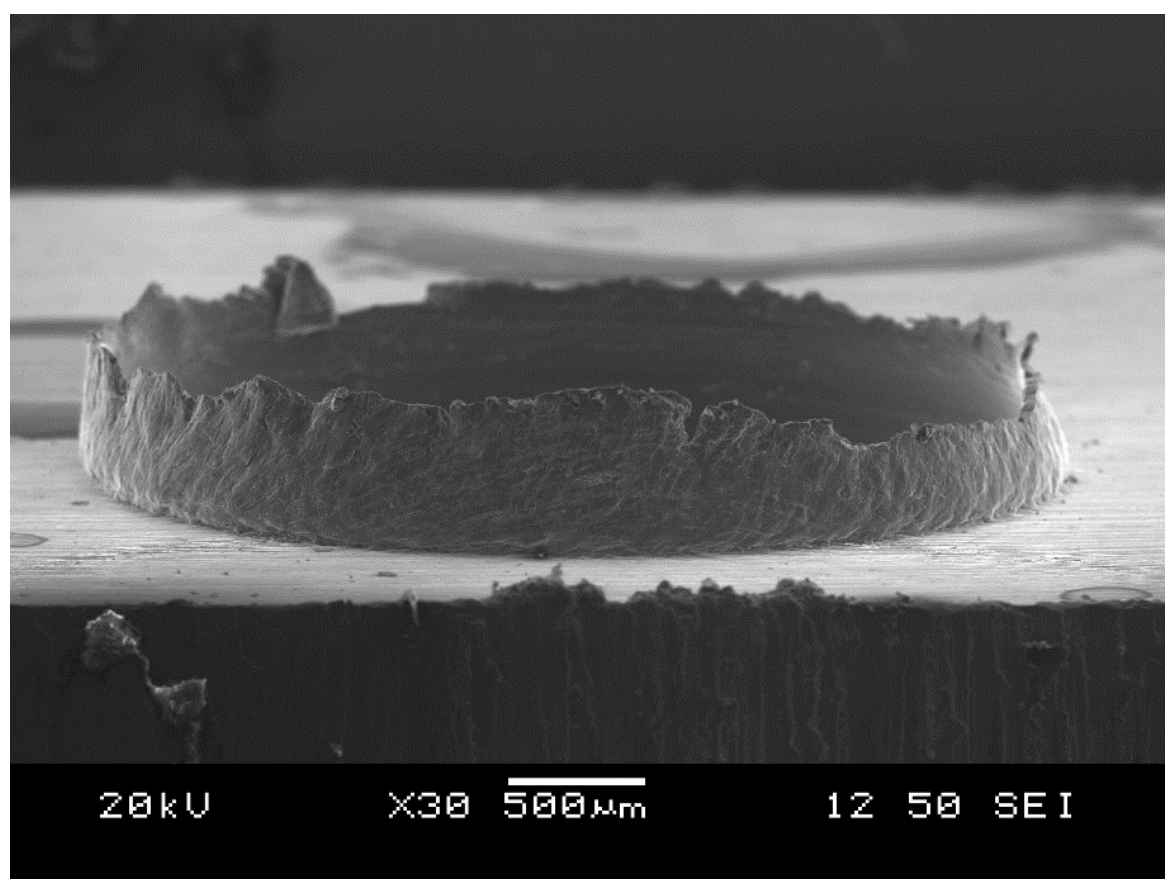

Fig. 5.9 Burr generated from an Al 6061 T6, .090"-thick plate, drilled with a 50\% worn drill bit at .002 in/rev (sample 1 - L)

\subsubsection{Drilled Sample Measurement}

Strips with two holes were cut from each $6 " \times 6$ " metal plate and sliced into individual samples using a standard band saw, with two holes on each sample to accommodate the SEM field of view. Sharp edges from cutting the samples were hand deburred using sandpaper, so as to not obstruct the full view of the burr. 


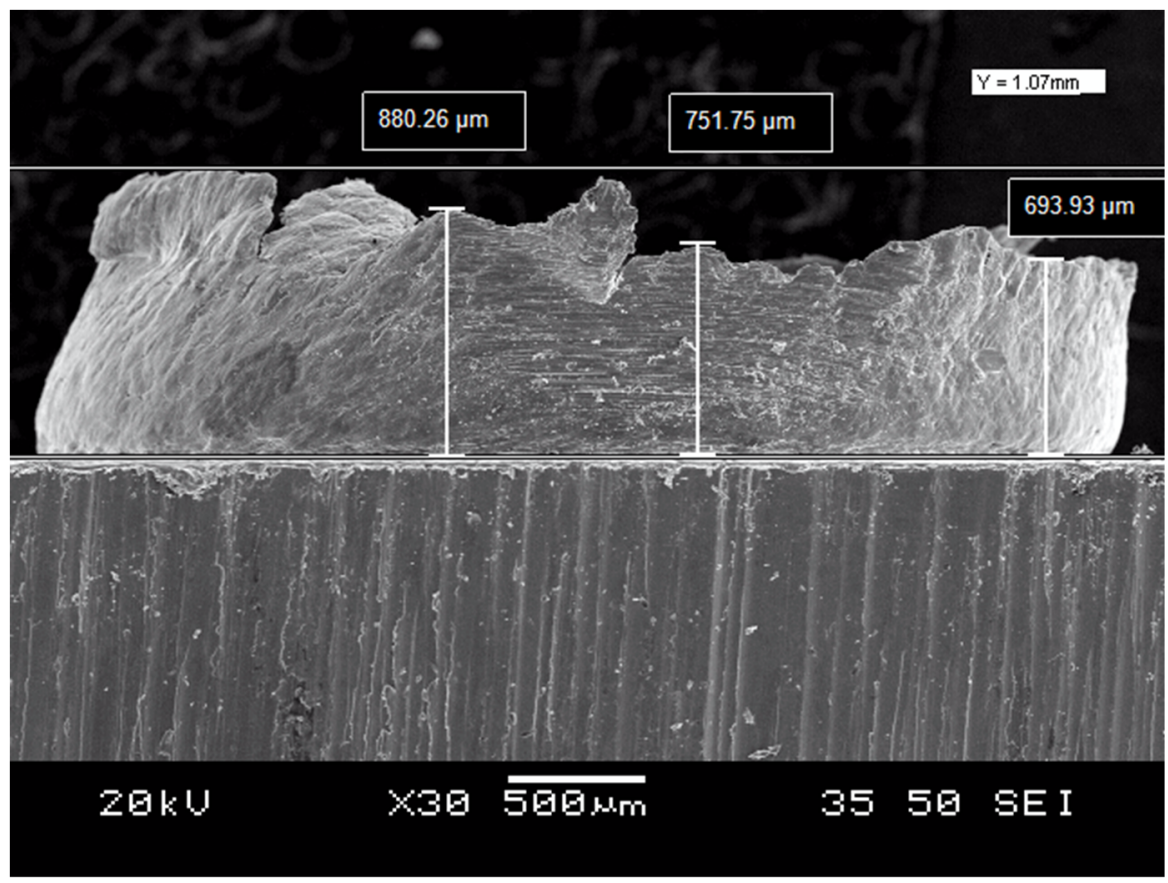

Fig. 5.10 Burr height measurement of burr generated from a plate of Al 6061 T6, .090" thick, drilled with a 50\% worn drill bit at $.004 \mathrm{in} / \mathrm{rev}$ (sample $14-\mathrm{L}$ )

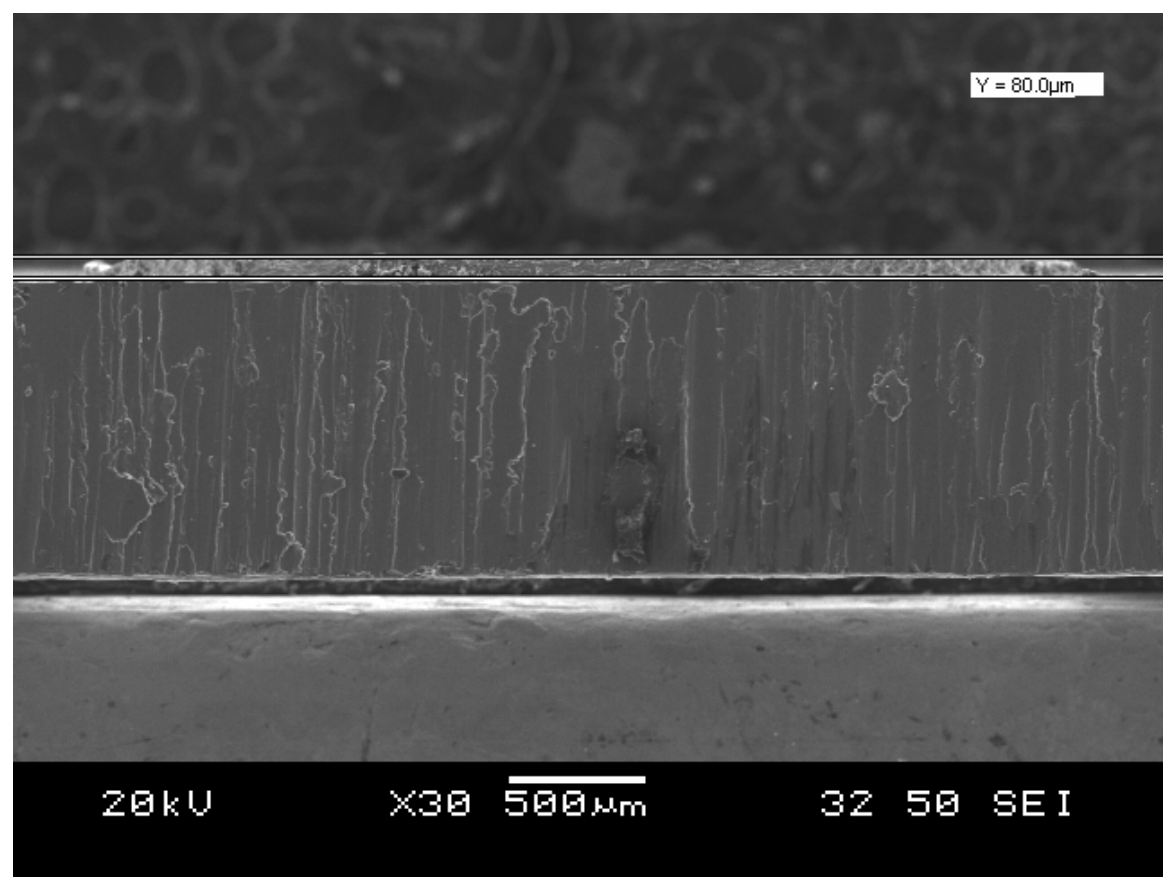

Fig. 5.11 Burr height measurement of burr generated from a plate of Al 6061 T6, .040" thick, drilled with a 0\% worn drill bit at $.004 \mathrm{in} / \mathrm{rev}$ (sample $68-\mathrm{R}$ )

Two hole measurements were made per each set of experimental conditions. Calibration was not required prior to measurements as it is done by the SEM manufacturer every two years, which had been 
completed within an industry accepted standard for SEM calibration. The samples were mounted perpendicular to the field of view to accommodate viewing and measurement. Burr thickness analysis was performed by mounting the samples burr side up, enabling a "bird's eye view" of the burr from the top and an appropriate zoom to view and measure the burr thickness. The highest point of the front half of the burr was always visible. Even if the highest point was not on the front surface of the burr, the projection of the highest point was visible and always measured. The high point of the burr height at the back of the sample was not always focused, but obtaining the correct height measurement was always possible.

When measuring, the sample was tilted to check the other half of the hole to ensure no significant burr height value was missed. If it was, the sample was taken out and turned around to ensure the lowest height was visible so that it could be measured. Burr height was measured at a range of 30-35x magnification. The visible bottom of the burr was indicated as the burr root. The number of measurements made depended on the burr formed. If the burr height was uniform throughout, as in Fig. 5.11, one burr height measurement was made at the level of the average burr height visible by the microscope. If the burr height was non-uniform, as in Fig. 5.10, additional measurements were made to capture the range of heights visible by the SEM, and an average burr height was calculated based on four measurements most representative of the range of burr height variance. The calculated average height was then assumed for the entire circumference of the burr. 


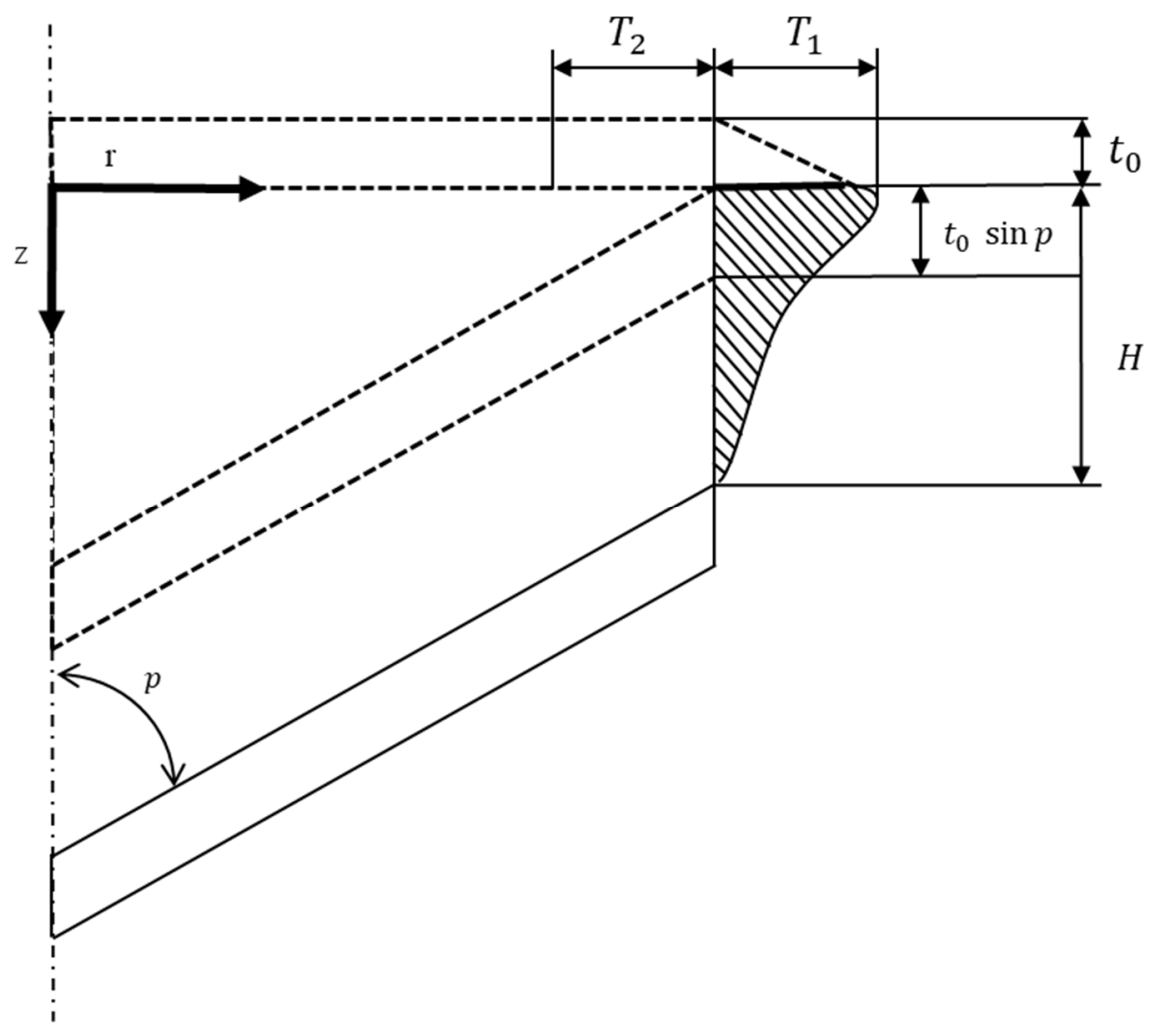

Fig. 5.12 Positive and negative burr thickness assignation

Burr thickness had to be split up into positive and negative burr thicknesses, as portrayed in Fig. 5.12. Positive burr thickness was measured using the top view of the burr, as it extended upwards from the burr root, as can be seen in Fig. 5.13 and Fig. 5.14. This burr measurement was only possible when the hole was completely drilled through. Positive burr thickness measurement was achieved when using 300-500x magnification, since thickness measurements ranged from $14.10 \mu \mathrm{m}$ to $133 \mu \mathrm{m}$. 


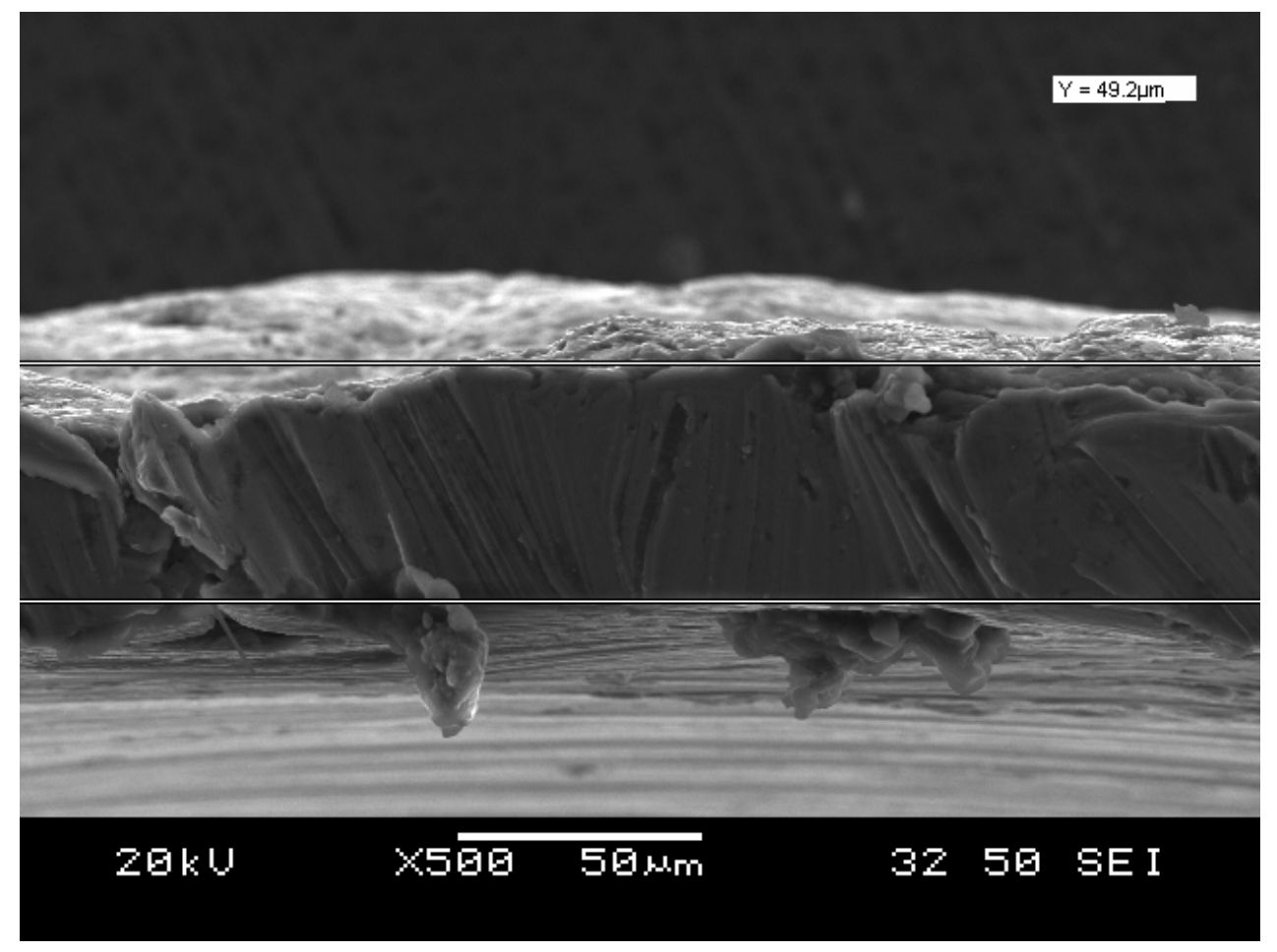

Fig. 5.13 Burr thickness measurement of burr generated from drilling a Al $6061 \mathrm{T6}, .090$ " thick plate, with a $50 \%$ worn drill bit at $.004 \mathrm{in} / \mathrm{rev}$ (sample $14-\mathrm{L}$ )

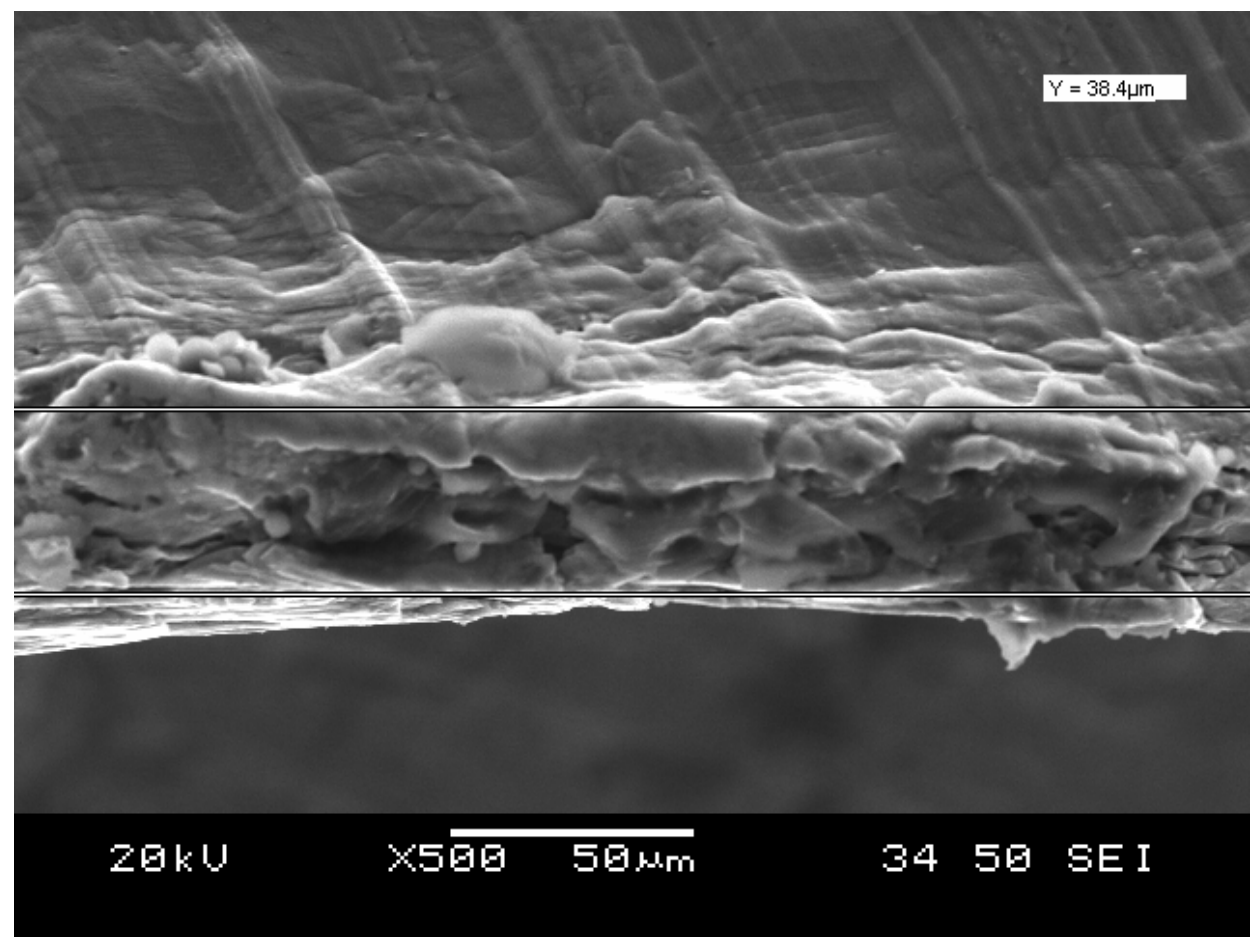

Fig. 5.14 Burr thickness measurement of burr generated from drilling a Al $6061 \mathrm{T6}, .040$ " thick plate, with a $50 \%$ worn drill bit at .004 in/rev (sample 9-L) 


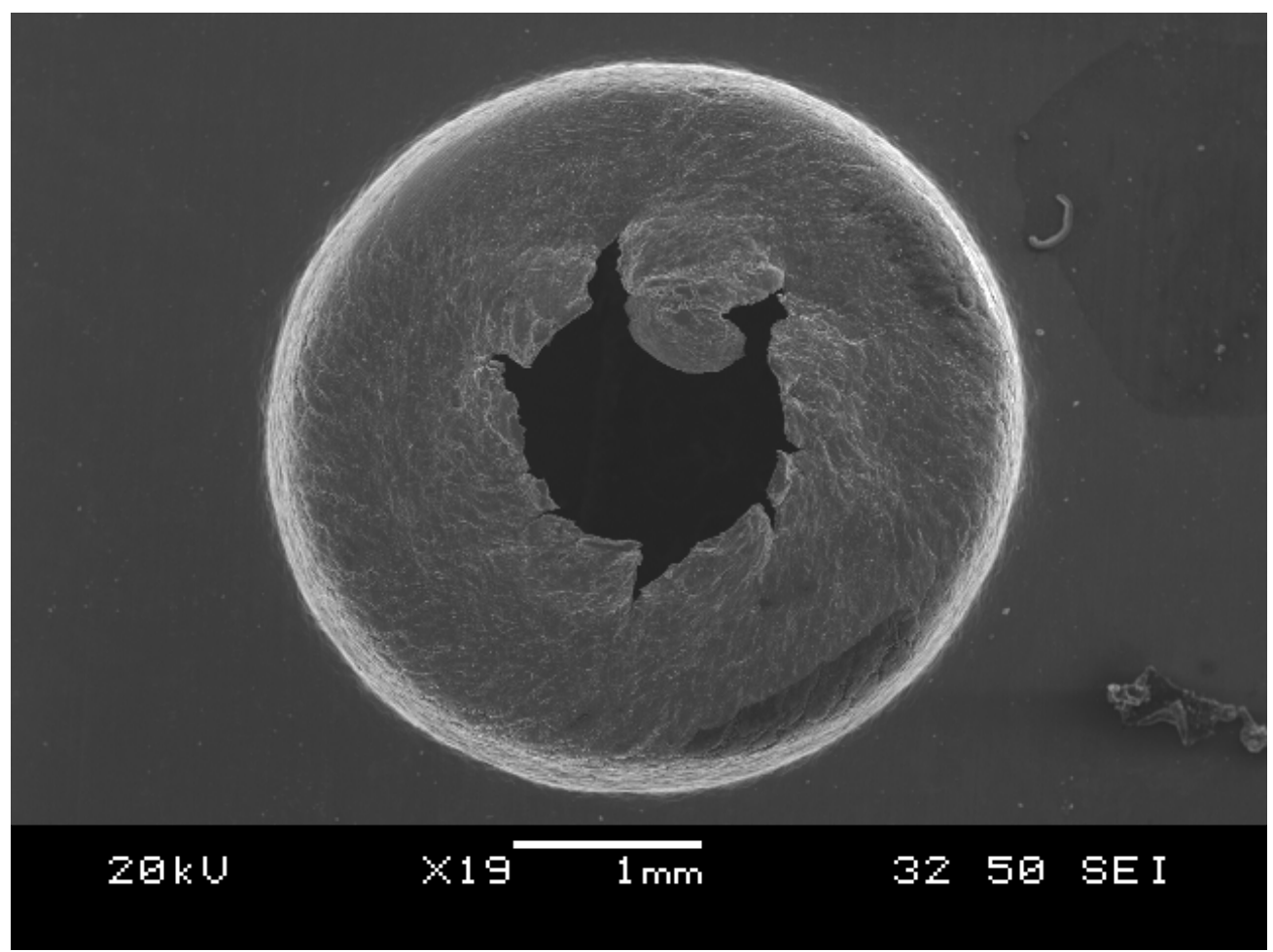

Fig. 5.15 Burr thickness, top view of burr generated from drilling a Al 6061 T6, .090" thick plate with a $75 \%$ worn drill bit at $.006 \mathrm{in} / \mathrm{rev}$ (sample $40-\mathrm{L}$ )

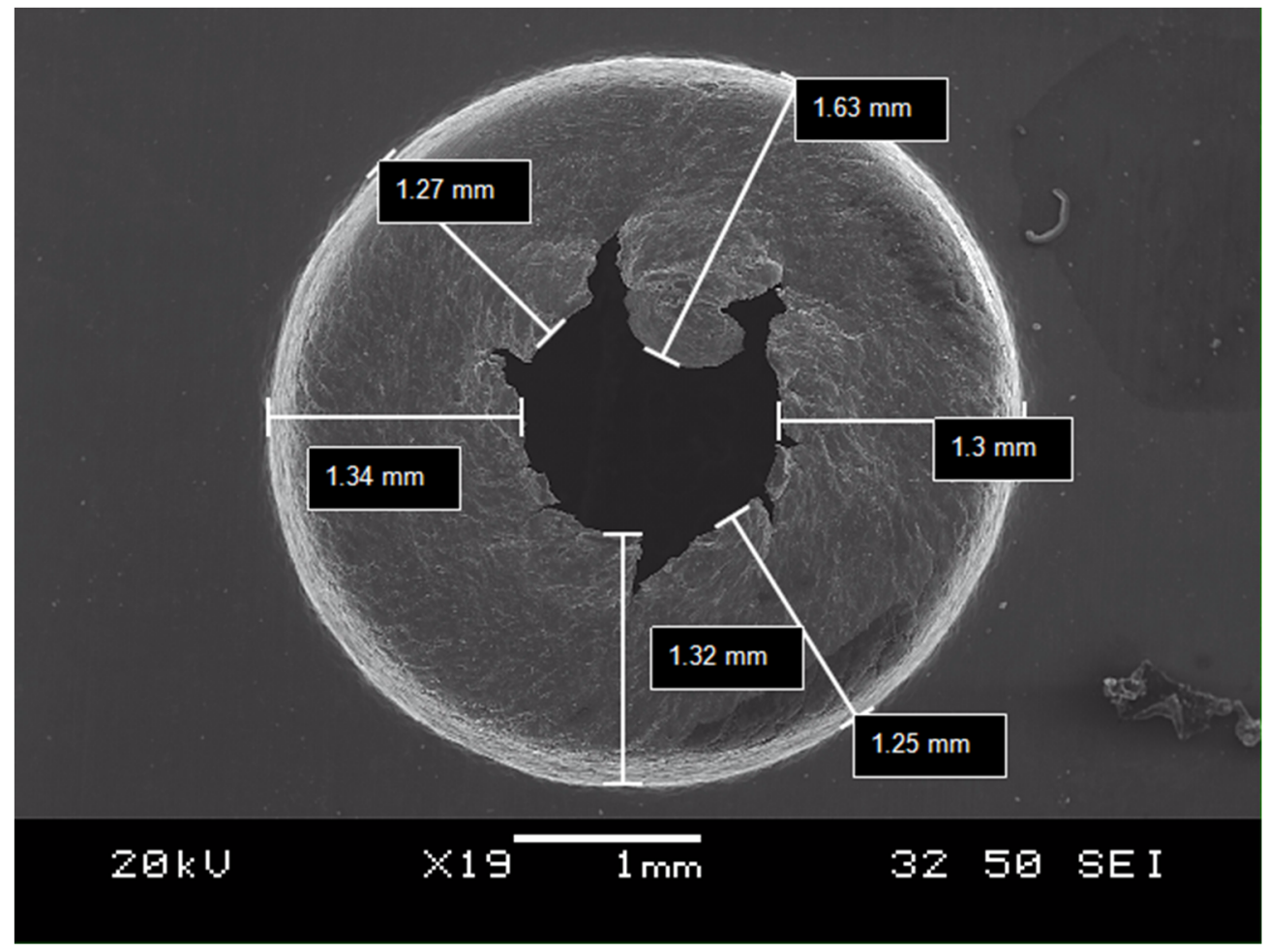

Fig. 5.16 Burr thickness, top view of burr generated from drilling a Al 6061 T6, .090" thick plate with a 75\% worn drill bit at $.006 \mathrm{in} / \mathrm{rev}$ (sample $40-\mathrm{L}$ ) 
When drilling with worn drill bits at $75 \%$ wear and greater, due to the increased ploughing thrust force, 84 holes in total were not penetrated and a cup-like burr remained, as shown in Fig. 5.15. This also occurred for 6 holes drilled with drill bits 50\% worn. In those cases, it was impossible for the SEM to measure the burr thickness because of this cup shape. Therefore, the distance from the outer edge of the cup to the end of the burr where the hole was drilled through was measured and interpreted as a negative burr thickness, as it formed in the direction opposite to that of a burr resulting from a nonploughing operation, as shown in Fig. 5.16.

Table 5.3 represents the quantity of measurements made for burr and thickness for AL 6061 T6, at a feed of .002 in/rev. This applies to all subsequent test cases for each of the three feeds, for both AL 6061 T6 and AL 7075 T6. For each of the three feeds and each of the two materials tested, 50 holes were measured for both burr height and thickness. Therefore, 25 holes were measured per each of the two material thicknesses, .040" and .090". In summary, a total of 300 holes were measured for burr height and burr thickness.

Table 5.3 Al 6061 T6 drilled at feed .002 in/rev - hole quantity

\begin{tabular}{|c|c|c|c|}
\hline Drill-bit wear \% & $\begin{array}{c}\text { Material } \\
\text { thickness [in] }\end{array}$ & $\begin{array}{c}\text { Hole quantity } \\
\text { per material } \\
\text { thickness }\end{array}$ & $\begin{array}{c}\text { Total \# of holes per } \\
\text { \% wear* }\end{array}$ \\
\hline \multirow{2}{*}{0} & 0.04 & 5 & \multirow{2}{*}{10} \\
\hline & 0.09 & 5 & \\
\hline \multirow{2}{*}{25} & 0.04 & 5 & \multirow{2}{*}{10} \\
\hline & 0.09 & 5 & \\
\hline \multirow{2}{*}{50} & 0.04 & 5 & \multirow{2}{*}{10} \\
\hline & 0.09 & 5 & \\
\hline \multirow{2}{*}{75} & 0.04 & 5 & \multirow{2}{*}{10} \\
\hline & 0.09 & 5 & \\
\hline \multirow{2}{*}{100} & 0.04 & 5 & \multirow{2}{*}{10} \\
\hline & 0.09 & 5 & \\
\hline
\end{tabular}

*A total of 50 holes were drilled measured, 25 per each material thickness

\subsection{Data Evaluation and Discussion}

Average heights and thicknesses, and standard deviations were calculated based on experimental data for each material, feed, percentage of wear, and plate thickness combination.

Figs. 5.17-5.20 show plots of burr height, thickness averages, and standard deviations with error bars. 
Experimental mean burr heights and thicknesses were compared with their corresponding simulation results. Green data points in the plots represent experimental data from burrs drilled in a .040" thick plate; red data points represent data from burrs drilled in a $.090 "$ thick plate. The blue continuous line with circles represents simulation results.

The error bars represent the variability of experimental data for each level of wear and feed. The centre of each error bar represents the calculated mean of recorded experimental burr height or thickness values for a given level of wear and feed. The upper and lower boundaries of the error bars represent the standard deviation of the population of burr heights and thicknesses, quantifying the amount of variation of the set of experimental data values. The standard deviation of a "population" was used as the experimental data are considered a population of experimental burr height and thickness data points of their own; it was not a sample representing a larger population of burr heights and thicknesses [55].

Experimental and calculated average heights and thicknesses from each set of drilling characteristics can be found in Appendix G.

As detailed in Section 4.3, eq. (4.16) and in accordance with the new burr height and thickness formation model, each percentage of drill bit wear indicates ploughing at that percent wear and is balanced out by a percentage of cutting occurring, adding up to a total of $100 \%$, that is, a $50 \%$ worn drill bit means $50 \%$ ploughing and $50 \%$ cutting is occurring.

Sections 5.2.1-5.2.4 provide a detailed description of the experimental and simulation results for Al 6061 T6 and Al 7075 T6 burr heights and thicknesses. Rationale explaining the variances between these results can be found in Section 5.4.

\subsubsection{AL 6061 T6 Height Analysis}

Fig. 5.17(a): At a feed of .002 in/rev, there were large error bars for all levels of wear, except for $0 \%$, which shows the smallest spread.

From $0 \%$ to $50 \%$ wear, there was a closer correlation between experimental and simulation data than at $75 \%$ and $100 \%$ wear. Between $0 \%$ and $50 \%$ drill bit wear, the experimental data spread averaged 107.25. At $0 \%$ and $25 \%$ drill bit wear, average experimental burr heights were, on average, $123.18 \mu \mathrm{m}$ and $274.93 \mu \mathrm{m}$, respectively, higher than the corresponding calculated burr heights. At $50 \%$ drill bit wear, all burr heights were, on average, $361.03 \mu \mathrm{m}$ higher than the corresponding calculated burr heights. 
Between $75 \%$ and $100 \%$ drill bit wear, the data spread averaged 108.57. The mean experimental burr heights were, on average, $262.41 \mu \mathrm{m}$ higher than the corresponding calculated burr heights. All heights were higher than those recorded in the simulation. The experimental data at this feed showed a more stochastic pattern. The overall burr growth trend agreed with the simulation data: the mean burr heights increased with increased wear, and were all, on average, $256.79 \mu \mathrm{m}$ higher than the calculated heights.

Fig. 5.17(b): At a feed of $.004 \mathrm{in} / \mathrm{rev}$, the error bars for burr heights recorded at $0 \%$ and $25 \%$ wear were smaller than those recorded at $.002 \mathrm{in} / \mathrm{rev}$. The data spread ranged from 7.94 to 61.51 and averaged 30.28. The mean experimental burr heights were, on average, between $10.91 \mu \mathrm{m}$ and $122.6 \mu \mathrm{m}$ higher than simulation values, averaging $73.17 \mu \mathrm{m}$ higher. At 50\% wear, there was a larger data spread, ranging from 181.81 to 531.26 and averaging 356.53. The mean burr heights were, on average, $468.53 \mu \mathrm{m}$ higher than simulation values; two of ten holes had not been not drilled through.

At $75 \%$ and $100 \%$ wear, the data spread ranged from 80.57 to 275.64 , averaging 147.33 . The mean burr heights averaged from $713.41 \mu \mathrm{m}$ to $1113.81 \mu \mathrm{m}$ higher than simulation values. They were, on average, $906.39 \mu \mathrm{m}$ higher. None of the holes drilled with $75 \%$ and $100 \%$ worn drill bits were completely penetrated by the drill, which generated very high burrs. The overall burr growth trend for holes drilled at $.004 \mathrm{in} / \mathrm{rev}$ agreed with the calculated burr height values as burr heights increased with increased drill bit wear; the overall experimental burr height increased at the higher feed.

Fig. 5.17(c): At a feed of $.006 \mathrm{in} / \mathrm{rev}$, the error bars for mean burr heights recorded at $0 \%$ and $25 \%$ ranged from 15.97 to 147.92, averaging 81.25; burr heights were between $13.93 \mu \mathrm{m}$ and $285.21 \mu \mathrm{m}$ higher than simulation values, and on average, they were $116.26 \mu \mathrm{m}$ higher. The data acquired when drilling with a 50\% worn drill bit had a spread ranging from 27.89 to 374.6. Burr height differences ranged from $221.20 \mu \mathrm{m}$ to $999.12 \mu \mathrm{m}$ between simulation and experimental values, averaging 610.16 $\mu \mathrm{m}$. Six of the ten holes had not been not completely drilled through, which generated very high burrs. This contributed to the difference between experiment and simulation data.

At $75 \%$ and $100 \%$ wear, the data spread was smaller, ranging from 49.31 to 118.33 , and just as at .004 in/rev, none of the holes drilled at this level of wear were drilled through. The mean burr height differences ranged from $1059.09 \mu \mathrm{m}$ to $1122.76 \mu \mathrm{m}$ between simulation and experimental values, experimental values averaging $888.19 \mu \mathrm{m}$ higher than calculated ones. 
Summing up the AL 6061 heights, minimum experimental burr heights occurred at 0\% and 25\% wear at all three feeds. The maximum burr heights were measured at $75 \%$ and $100 \%$ wear for all three feeds. The delta between experimental and simulation burr heights at these levels of drill bit wear at .004 in/rev and $.006 \mathrm{in} / \mathrm{rev}$ were approximately twice the difference at 50\% wear and at a minimum five times the deltas at $0 \%$ and $25 \%$ drill bit wear. Experimental data demonstrated better agreement with calculated values for holes drilled at $0 \%$ to $50 \%$ drill bit wear than those at $75 \%$ to $100 \%$ wear at .004 in/rev and $.006 \mathrm{in} / \mathrm{rev}$. At $.002 \mathrm{in} / \mathrm{rev}$, experimental data demonstrated a more stochastic pattern and did not follow this trend. Material thickness did not play a role in burr growth trends; there was no associated pattern noted.

\subsubsection{AL 6061 T6 Burr Thickness Analysis}

Fig. 5.18(a): At $.002 \mathrm{in} / \mathrm{rev}$, there was a minimal spread in the data for the burr thicknesses. The standard deviation ranged from 2.67 to 9.88 for all levels of wear. The measured thicknesses ranged, on average, between $22.2 \mu \mathrm{m}$ and $38.63 \mu \mathrm{m}$, and they did not manifest a gradual increase in thickness with respect to increased tool wear. At $0 \%, 25 \%$, and $50 \%$ drill bit wear, the experimental burr thicknesses were, on average, $9.08 \mu \mathrm{m}, 17.37 \mu \mathrm{m}$, and $53.7 \mu \mathrm{m}$ lower, respectively, than the corresponding calculated burr thicknesses. At $75 \%$ and $100 \%$ drill bit wear, experimental burr thicknesses were, on average, $147.68 \mu \mathrm{m}$ and $255.08 \mu \mathrm{m}$ lower, respectively, than the corresponding calculated burr thicknesses. This increased divergence between simulated and experimental data can be attributed to the experimental thicknesses remaining at less than $50 \mu \mathrm{m}$. Stochastic burr behaviour, with respect to Al 6061 T6 experimental burr heights at a feed of .002 in/rev, manifested for the thicknesses as well.

Fig. 5.18(b): At a feed of $.004 \mathrm{in} / \mathrm{rev}$, the data spread for burr thicknesses recorded at $0 \%, 25 \%$, and $50 \%$ was very small, the standard deviation ranging from 5.54 to 28.31 and averaging 12.88 . These sets of values were all lower than the calculated ones; the differences between measured and calculated burr thicknesses ranged, on average, between $36.22 \mu \mathrm{m}$ and $51.9 \mu \mathrm{m}$.

At $75 \%$ and $100 \%$ wear, the data spread averaged 370.51 . The burr thicknesses were negative at $75 \%$ and $100 \%$ as the drilled holes at these levels of wear were not completely penetrated. When looking at burr thicknesses at $75 \%$ and $100 \%$ wear (as shown in Fig. 5.12; thicknesses for non-penetrated burrs were assigned a negative value), the differences between mean simulated and measured values ranged from $142.96 \mu \mathrm{m}$ to $1712.03 \mu \mathrm{m}$. Despite the significant differences between burr thicknesses, growth trends for holes drilled at $.004 \mathrm{in} / \mathrm{rev}$ agreed with the calculated burr height values, as the overall experimental burr increased in thickness. 
Fig. 5.18(c): At a feed of $.006 \mathrm{in} / \mathrm{rev}$, the data spread for burr thicknesses recorded at $0 \%$ and $25 \%$ wear was very small, ranging from 11.29 to 21.38 . At $0 \%$ and $25 \%$, the thicknesses were, on average, 60.23 $\mu \mathrm{m}$ lower than the calculated values. The trend once again was sustained. The experimental thicknesses were lower than the simulation ones, but at the same time, with increased levels of wear, the burr thicknesses increased. At 50\%, the data spread increased to an average of $369.93 .60 \%$ of the drilled holes were not penetrated, and the average burr height differed by $692.12 \mu \mathrm{m}$ from calculated values due to the negative burr height assignation.

Between $75 \%$ and $100 \%$ wear, the recorded thickness data spread was, on average, $143.58 \mu \mathrm{m}$. Burr thicknesses were all negative at these levels of wear, as all of the drilled holes were non-penetrated. From $75 \%$ to $100 \%$ wear, the difference between simulated and measured thicknesses was, on average, 1,628.82 $\mu \mathrm{m}$. Despite the significant differences between experimental and simulated values, the overall burr thickness increased at the higher feed, and the burr thickness increased with increased drill bit wear.

The results revealed variance for burr thicknesses for AL 6061 between experimental and calculated results. At $.002 \mathrm{in} / \mathrm{rev}$, a more stochastic behaviour was observed. A consistent trend was that most thicknesses recorded were less than those generated by the simulation.

For all three feeds, the thicknesses showed general agreement between the simulation and experimental results up until drill bit wear was greater than $50 \%$. However, there were some outliers. As mentioned, for the feed of $.002 \mathrm{in} / \mathrm{rev}$, all burr thicknesses were lower than the calculated values, did not gradually increase with wear, and remained within the average range of $22 \mu \mathrm{m}$ to $38.63 \mu \mathrm{m}$. This demonstrates stochastic behaviour, also observed for burr heights at the same feed. For the feed of .006 $\mathrm{rev} / \mathrm{in}$ at $50 \%$ wear, the data spread increased to an average of 369.93 , and at $0 \%$ and $25 \%$ wear it averaged 14.80 , as $60 \%$ of the drilled holes were not penetrated.

Between $75 \%$ and $100 \%$ wear, a larger variance could be observed, particularly for $.004 \mathrm{in} / \mathrm{rev}$ and .006 in/rev feeds, because the burr thickness was negative, meaning the holes were not completely drilled through and only a negative thickness could be measured. Material thickness was of no consequence, irrespective of feed. 


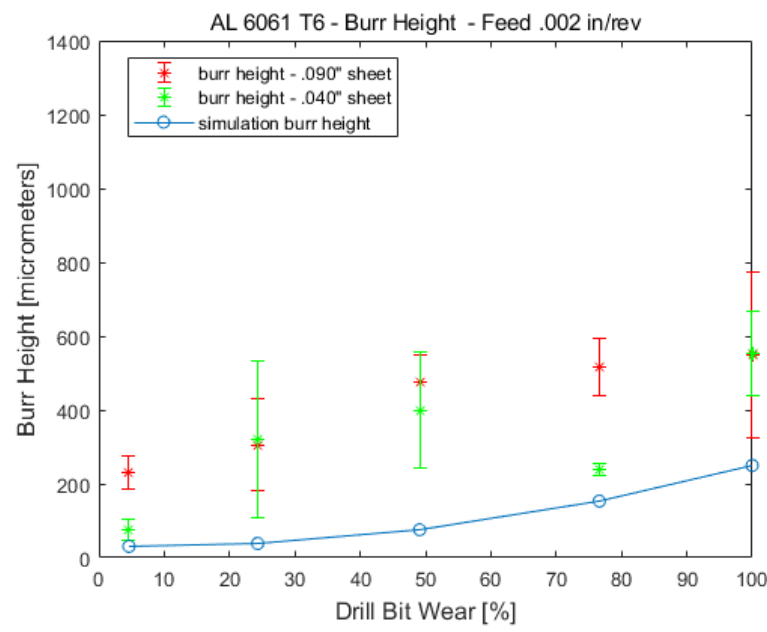

(a)

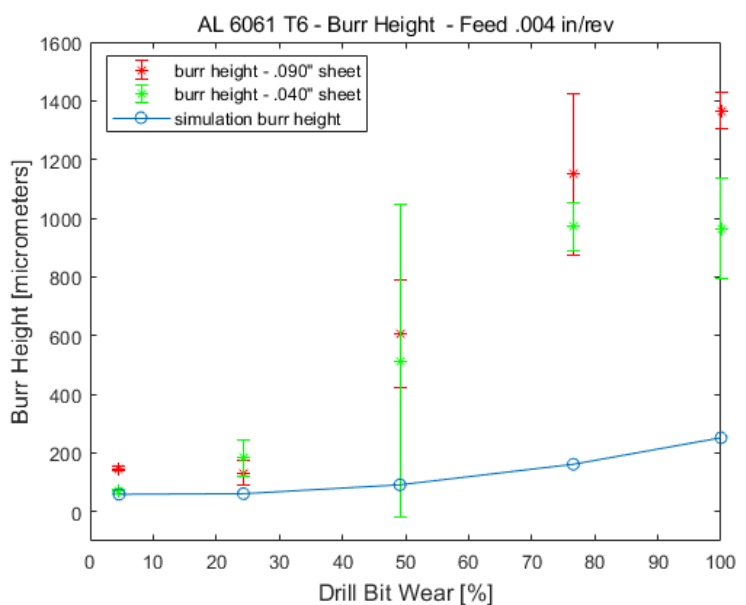

(b)

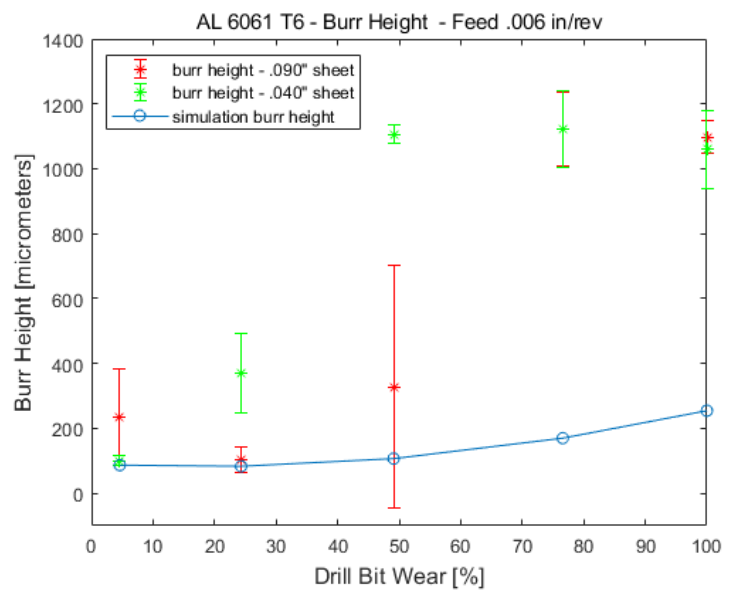

(c)

Fig. $5.17(\mathrm{a}-\mathrm{c})$ Burr height [ $\mu \mathrm{m}]$ in relation to drill-bit wear [\%] resulting from drilling Al $6061 \mathrm{T6}$. The red error bars represent burr heights drilled in .090" thick sheets, the green error bars represent burr heights drilled in .040" thick sheets, and the blue line with circles shows the calculated burr heights. 


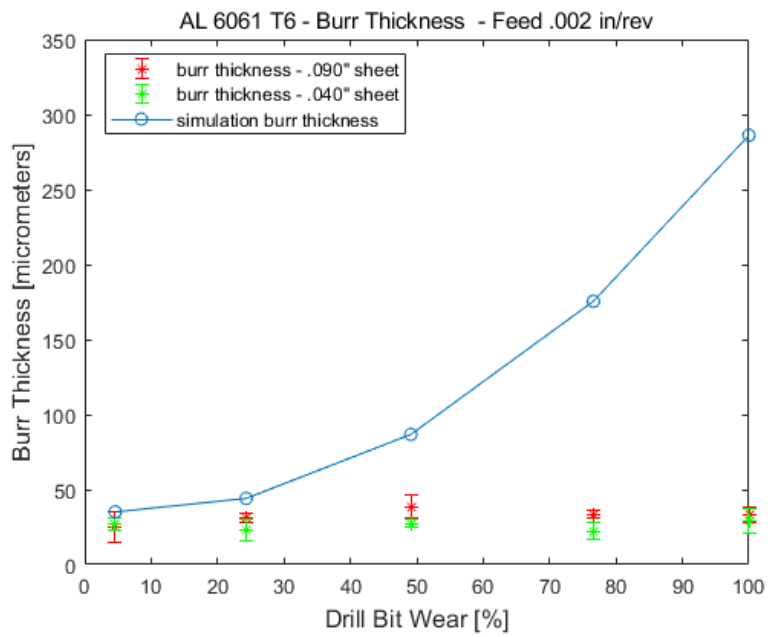

(a)

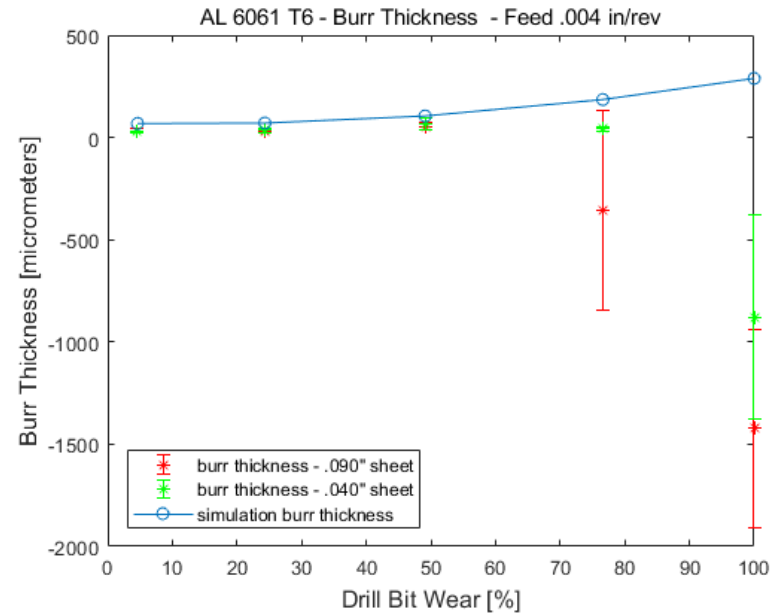

(b)

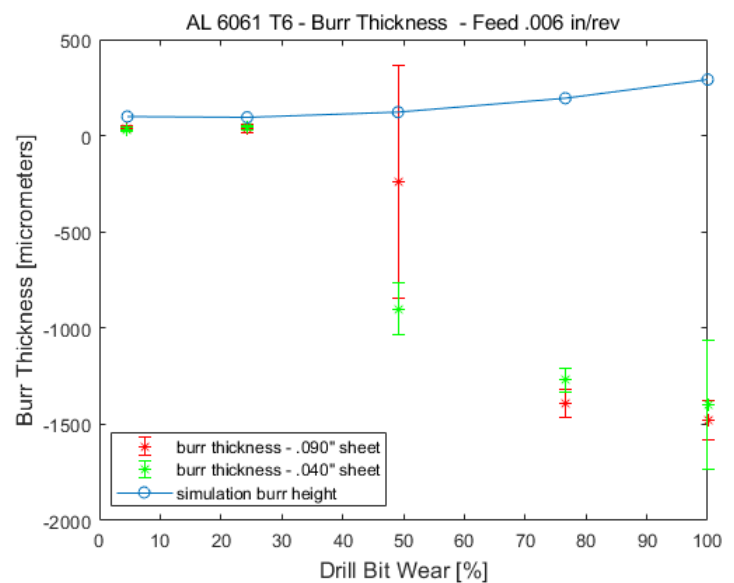

(c)

Fig. $5.18(\mathrm{a}-\mathrm{c})$ Burr thickness [ $\mu \mathrm{m}]$ in relation to drill-bit wear [\%] resulting from drilling Al $6061 \mathrm{T6}$. The red error bars represent burr heights drilled in .090" thick sheets, the green error bars represent burr heights drilled in .040" thick sheets, and the blue line with circles shows calculated burr heights. 


\subsubsection{AL 7075 T6 Burr Height Analysis}

Fig. 5.19(a): At a feed of .002 in/rev, from $0 \%$ to $50 \%$ wear there was a closer correlation observed between experimental and simulation data than at $75 \%$ and $100 \%$.

For $0 \%$ and $25 \%$, the data spread was, on average, 47.64. Differences between mean experimental and calculated values ranged from $47.80 \mu \mathrm{m}$ to $209 \mu \mathrm{m}$, mean experimental values averaging $121.37 \mu \mathrm{m}$ higher than the calculated ones. For $50 \%$ wear, the mean measured values were within $15 \mu \mathrm{m}$ of the calculated burr heights, and the average data spread was 16.61. For holes drilled in a .090" thick sheet, four out of five holes had burrs lower than those predicted by the model. For holes drilled in a .040" thick sheet, three out of five burr heights were lower than those calculated by the model.

For $0 \%$ to $50 \%$ wear, the average data spread was 37.30 , and the mean burr heights were on average $79.76 \mu \mathrm{m}$ higher than the calculated ones. All holes drilled at these levels of drill bit wear were completely penetrated.

At $75 \%$ drill bit wear, the mean measured burr heights were, on average, $1284.93 \mu \mathrm{m}$ higher than the calculated values; and the average data spread was 79.39 . At $100 \%$ wear, two different patterns emerged. The mean burr heights drilled in $.090 "$ thick material were $709.70 \mu \mathrm{m}$ higher than the simulated heights. Those drilled in .040 " thick material were $102.84 \mu \mathrm{m}$ lower than the calculated values, and the holes were fully penetrated. Aside from this one data subset, all the holes at $75 \%$ and $100 \%$ were non-penetrated. The one instance when the trend differed with respect to material thickness was an outlier.

The new burr formation model more adequately predicted burr heights for $0 \%$ to $50 \%$ wear than for $75 \%$ and $100 \%$ wear.

The general trend observed for measured versus calculated values for $.002 \mathrm{in} / \mathrm{rev}$ indicated that simulated and measured values increased with the level of wear.

Fig. 5.19(b): At a feed of $.004 \mathrm{in} / \mathrm{rev}$, the error bars for burr heights recorded at $0 \%$ to $50 \%$ wear averaged a spread of 31.32. These sets of mean experimental values ranged from $41.80 \mu \mathrm{m}$ to $86.55 \mu \mathrm{m}$ higher than the calculated values and were on average $63.57 \mu \mathrm{m}$ higher. All holes drilled with these levels of drill bit wear were completely penetrated.

At $75 \%$ and $100 \%$ wear, the data spread was smaller than the $0 \%$ to $50 \%$ wear, averaging 48.92 , but the measured burr heights were, on average, $1048.21 \mu \mathrm{m}$ higher than simulation values. The differences 
between mean experimental and calculated burr heights ranged from $858.11 \mu \mathrm{m}$ to $1169.53 \mu \mathrm{m}$. Like the burrs from holes drilled at $.002 \mathrm{in} / \mathrm{rev}$ at these levels of wear, none of the holes drilled with $75 \%$ and $100 \%$ worn drill bits were completely penetrated by the drill, and as a result, they generated very high burrs. The overall burr growth trend for holes drilled at $.004 \mathrm{in} / \mathrm{rev}$ agreed with the calculated burr height values. The burr height increased with increased drill bit wear.

Fig. 5.19(c): At a feed of $.006 \mathrm{in} / \mathrm{rev}$ the error bars for burr heights recorded at $0 \%, 25 \%$, and $50 \%$ averaged 38.92. The mean measured burr height values were, on average, $61.87 \mu \mathrm{m}$ higher than the calculated ones. The differences ranged from $18.77 \mu \mathrm{m}$ to $137.24 \mu \mathrm{m}$. All holes measured at these levels of wear were completely penetrated.

At $75 \%$ and $100 \%$ wear, the data spread was approximately twice that of $0 \%$ to $50 \%$ wear, averaging 67.27. The burr height values ranged from $933.00 \mu \mathrm{m}$ to $1378.61 \mu \mathrm{m}$. The mean experimental burr height for these levels of wear was $1176 \mu \mathrm{m}$ which is on average 19x higher than burr heights at $0 \%$ to $50 \%$ wear. Similar to burr heights for feeds of $.002 \mathrm{in} / \mathrm{rev}$ and $.004 \mathrm{in} / \mathrm{rev}$, none of the holes drilled at a $75 \%$ or $100 \%$ level of drill bit wear were drilled through.

In summary, for Al 7075 T6 the minimum experimental burr heights were consistently observed at $0 \%$ and $25 \%$ wear at all three feeds. Experimental burr heights at $0 \%$ to $50 \%$ wear demonstrated closer agreement with calculated values, while at $75 \%$ and $100 \%$ they did not. The maximum burr heights were measured at $75 \%$ and $100 \%$ wear for all three feeds. The measured values at $75 \%$ and $100 \%$ drill bit wear were consistently at least $760 \mu \mathrm{m}$ greater than the calculated data. None of the holes at those levels of wear were penetrated, with the exception of the outlier at $.002 \mathrm{in} / \mathrm{rev}$ for .040 " thick material at $100 \%$ drill bit wear. The model did not show good agreement with experimental data at these higher levels of wear for any of the feeds. Material thickness did not play a role in burr growth trends, and there was no associated pattern noted.

\subsubsection{AL 7075 T6 Burr Thickness Analysis}

Fig. 5.20(a): At .002 in/rev, for $0 \%$ to $50 \%$ wear there was a minimal data spread observed for burr thicknesses, averaging 4.72. All thicknesses in this range were lower than the simulated value, and all were fully penetrated holes. The differences between measured and calculated values ranged between $9.10 \mu \mathrm{m}$ and $53.34 \mu \mathrm{m}$, averaging $28.11 \mu \mathrm{m}$.

At $75 \%$ and $100 \%$ wear, $75 \%$ of the holes were not drilled through and the recorded burr heights were consequently all negative. The data spread was larger, averaging 380.98. The mean measured 
thicknesses were on average $853.73 \mu \mathrm{m}$ higher than the calculated thicknesses, presenting significant divergence.

Experimental burr thicknesses followed an overall trend similar to Al 6061, where the measured burr thicknesses increased with the levels of wear. The measured values were in better agreement with experimental values at $0 \%$ to $50 \%$ wear and differed significantly at $75 \%$ and $100 \%$ wear.

Fig. 5.20(b): At a feed of $.004 \mathrm{in} / \mathrm{rev}$, the error bars for burr thicknesses recorded at $0 \%, 25 \%$, and $50 \%$ wear were very small, and the data spread was greater than at $.002 \mathrm{in} / \mathrm{rev}$ feed, averaging 9.87. The differences between the measured and calculated values ranged between $41.94 \mu \mathrm{m}$ and $74.24 \mu \mathrm{m}$ and averaged $52.57 \mu \mathrm{m}$.

At $75 \%$ and $100 \%$ wear, the data spread averaged 82.73 . Similar to holes drilled at a feed of $.002 \mathrm{in} / \mathrm{rev}$ at the same level of wear, burr thicknesses were negative, as none of the drilled holes at $75 \%$ and $100 \%$ wear were completely penetrated. The differences between the simulated and mean measured values ranged from $1429.93 \mu \mathrm{m}$ to $1946.67 \mu \mathrm{m}$, averaging $1618.71 \mu \mathrm{m}$. Burr thicknesses at this level of wear also followed the trend observed at the feed of $.002 \mathrm{in} / \mathrm{rev}$; there was a smaller divergence and therefore better agreement between burr thicknesses for holes drilled at $0 \%$ to $50 \%$ wear, while at $75 \%$ and $100 \%$ wear there was a large divergence between the calculated and experimental values.

The overall growth trend for holes drilled at $.004 \mathrm{in} / \mathrm{rev}$ followed that of $.002 \mathrm{in} / \mathrm{rev}$. The experimental burr thicknesses increased at the higher feed, and the burr thickness increased with increased drill bit wear.

Fig. 5.20(c): At a feed of $.006 \mathrm{in} / \mathrm{rev}$, the error bars for burr thicknesses recorded at $0 \%, 25 \%$, and $50 \%$ wear were small. The data spread was similar to that at $.004 \mathrm{in} / \mathrm{rev}$, averaging 9.53 . The differences between the measured and calculated values ranged similarly to that of $.004 \mathrm{in} / \mathrm{rev}$, between $65.30 \mu \mathrm{m}$ and 82.10, averaging $71.89 \mu \mathrm{m}$. All thicknesses were lower than the simulated values, and all the holes at this level of wear were fully penetrated.

At $75 \%$ and $100 \%$ wear, the recorded thickness data spread was much greater, averaging 85.27 . Burr thicknesses were negative at this feed. None of the drilled holes at these levels of wear were completely penetrated. At $75 \%$ and $100 \%$ wear, the differences between simulated and measured values were once again significant and also consistent with those measured at feeds of $.002 \mathrm{in} / \mathrm{rev}$ and $004 \mathrm{in} / \mathrm{rev}$. They ranged from $1485.07 \mu \mathrm{m}$ to $1976.63 \mu \mathrm{m}$, averaging $1704.43 \mu \mathrm{m}$. The model proved more reliable for predicting experimental values at $0 \%$ to $50 \%$ wear, and less reliable at $75 \%$ and $100 \%$ wear. 
Overall, the burr thickness increased at the higher feed and the burr thickness increased linearly with increased drill bit wear.

The minimum burr thickness once again occurred at $0 \%$ and $25 \%$ wear at all three feeds. The maximum burr heights were measured at $75 \%$ and $100 \%$ wear for all three feeds. Once again the trend was sustained: with increased levels of wear, the burr thickness increased. Overall, burr thicknesses consistently increased with an increased feed.

Material thickness, once again, did not play a role in burr growth trends; there was no associated pattern noted. 


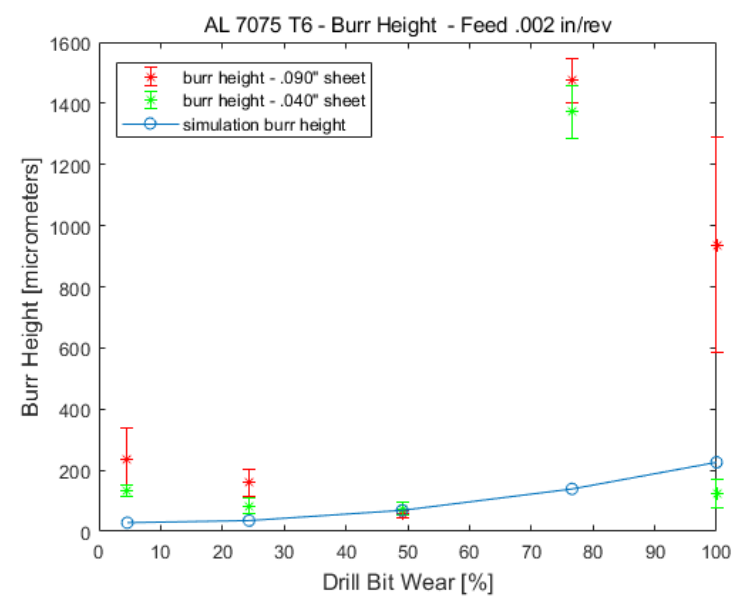

(a)

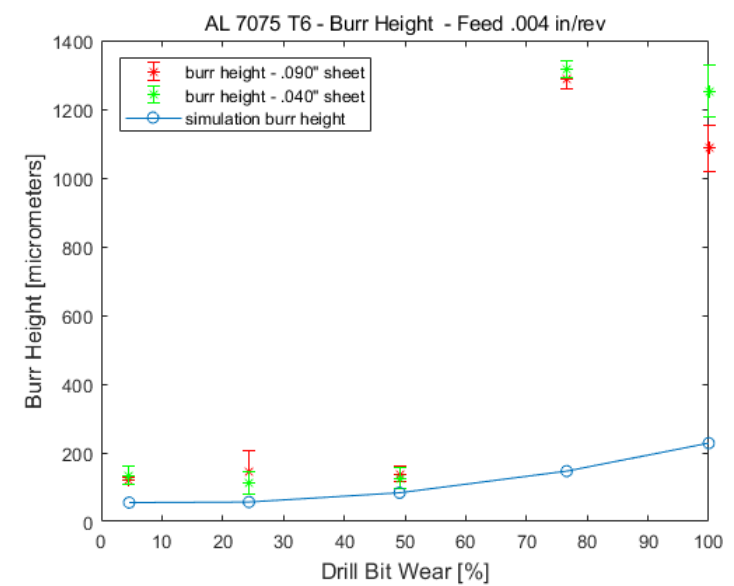

(b)

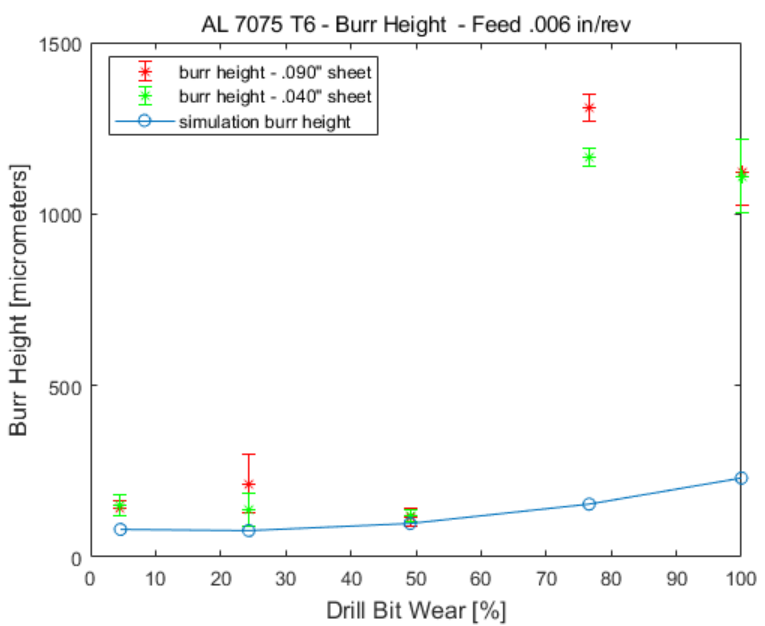

(c)

Fig. $5.19(\mathrm{a}-\mathrm{c})$ Burr height $[\mu \mathrm{m}]$ in relation to drill-bit wear [\%] resulting from drilling Al $7075 \mathrm{~T} 6$. The red error bars represent burr heights drilled in .090" thick sheets, the green error bars represent burr heights drilled in .040" thick sheets, and the blue line with circles shows calculated burr heights. 


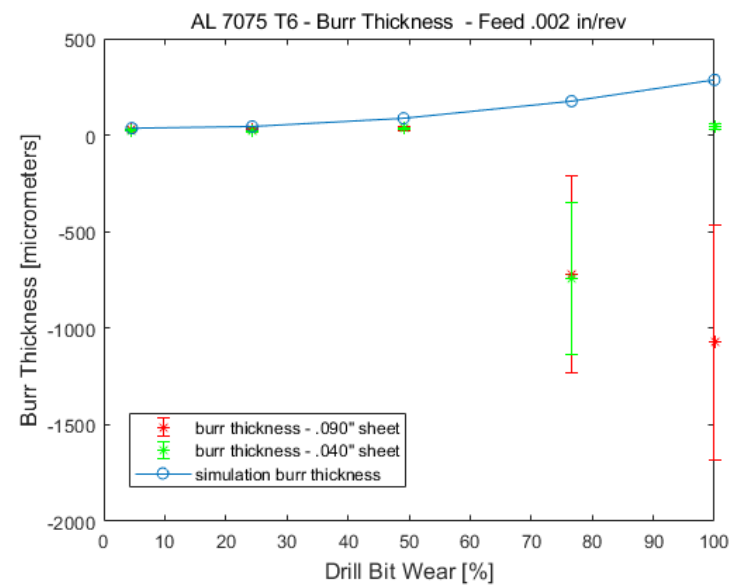

(a)

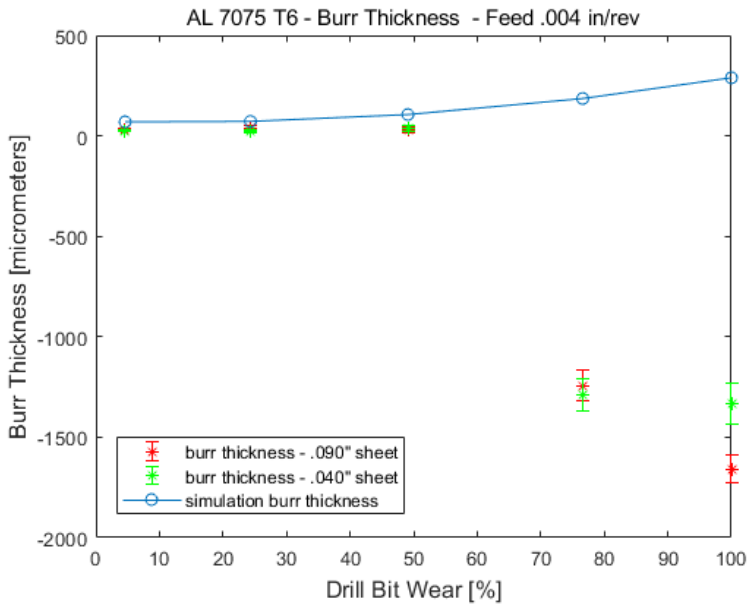

(b)

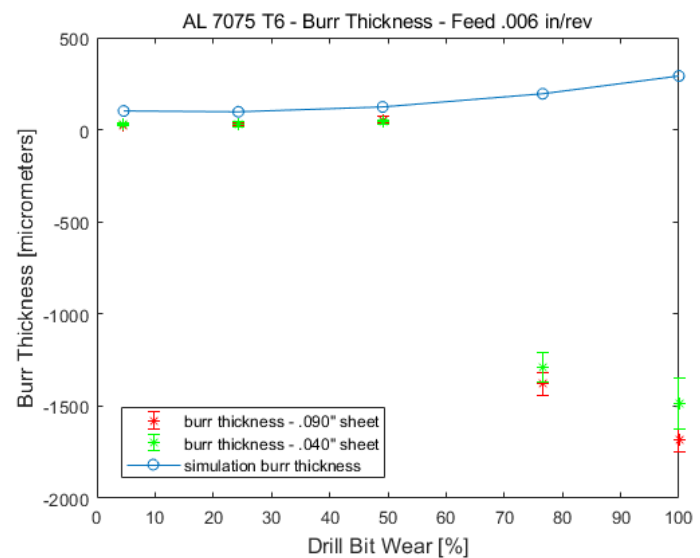

(c)

Fig. $5.20(\mathrm{a}-\mathrm{c})$ Burr thickness [ $\mu \mathrm{m}]$ in relation to drill-bit wear [\%] resulting from drilling Al $7075 \mathrm{T6}$. The red error bars represent burr heights drilled in .090" thick sheets, the green error bars represent burr heights drilled in .040" thick sheets, and the blue line with circles shows the calculated burr heights. 


\subsection{Additional Considerations Regarding Experimental Data}

When assessing the experimental data, it is important to address the causes and justifications for variance where collected data diverges from calculated results. These rationales are described below.

As discussed in sections 5.2.1-5.2.4, where comparisons are made between experimental and theoretical models, the new burr height and thickness model is generally able to predict burr height and thickness for $0 \%$ to $50 \%$ drill bit wear. The model is less able to do so for $75 \%$ and $100 \%$ wear.

\subsubsection{Mode of Drill Bit Wear}

The drill feature that was worn down and incorporated into the new burr formation model was the drill bit cutting edge. As described in Chapter 3, forces on the drill bit cutting edge were evaluated by treating the cutting mechanism as an oblique cutting process, stemming from orthogonal cutting, and approximating the cutting lip into individual segments. This sum represents the total drilling thrust force. The other part of the drill bit tip that contributed to the total drilling thrust force was the chisel edge, as detailed by Altintas [34]. The chisel does not perform a cutting action but, instead, performs an indentation action, moving the work material to the sides.

Divergence between experimental and theoretical results in this dissertation may partially stem from including only the drill bit cutting edge in the calculation of the drilling thrust force and not including the chisel edge. This is because chisel forces can be assumed to be about $10 \%-15 \%$ of the total lip cutting forces.

A basic indentation model will deliver inaccurate results in calculating the thrust force of the chisel edge. The intricacy of the chisel edge geometry and indentation action calls for experimental assessment and demands sophisticated geometric modeling. Incorporation of chisel edge wear in the burr formation model is a consideration for future expansion of the burr height and thickness model established in this dissertation. While this dissertation did not focus on flank wear, it is important to acknowledge that flank wear may have contributed to the difference between the experimental and calculated results. Given that flank wear occurs in all machining operations, this mode of wear likely contributed to the increased thrust forces and greater burr heights and thicknesses corresponding with the increased levels of drill bit wear. The inability of the new burr height and thickness model to align the burr growth at $75 \%$ and $100 \%$ wear with experimental results was possibly impacted by flank wear. The rate of flank wear varies with time [29]. Flank wear begins following initial cutting edge rounding and gradually 
increases to the point of critical land width. Once flank wear progresses past the critical land width it increases rapidly, reaching a level of severe wear.

\subsubsection{Modeling for Penetrated vs. Non-Penetrated Holes}

A key pattern was observed for both materials with regard to burr heights and thicknesses. Experimental heights and thicknesses aligned more closely to calculated values for $0 \%, 25 \%$ and $50 \%$ levels of wear, while at $75 \%$ and $100 \%$, at feeds of $.004 \mathrm{in} / \mathrm{rev}$ and $.006 \mathrm{in} / \mathrm{rev}$, most experimental values were greater by $710 \mu \mathrm{m}$ or more; these were heights and thicknesses from non-penetrated holes.

Given the closer alignment between experimental and simulation burr height and thickness results at $0 \%$ to $50 \%$ wear and the disparity at $75 \%$ to $100 \%$ wear, the current model adequately predicts burr growth at $0 \%$ to $50 \%$ drill bit wear and only predicts a trend at $75 \%$ to $100 \%$ drill bit wear. More specifically, while the model shows that burr heights and thicknesses will continue to increase with increased drill bit wear, the model is unable to forecast burr heights and thicknesses from non-penetrated holes.

For Al 6061 T6, at feeds of $.004 \mathrm{in} / \mathrm{rev}$ and $.006 \mathrm{in} / \mathrm{rev}, 32$ out of 40 samples drilled at $75 \%$ and $100 \%$ wear were not completely penetrated. At $0 \%$ to $50 \%$ wear, 6 out of 60 samples were not drilled through. (Details can be found in Appendix G, Section G.1.) Data obtained at $.002 \mathrm{in} / \mathrm{rev}$ is excluded from these numbers because of the stochastic behaviour, meaning that all the holes drilled at $.002 \mathrm{in} / \mathrm{rev}$ at $75 \%$ and $100 \%$ wear were completely penetrated, making them outliers in the data set.

For Al 7075 T6 at $75 \%$ to $100 \%$ wear, 52 out of 60 samples were not penetrated. At $0 \%$ to $50 \%$ wear, all the samples were drilled through. (Details can be found in Appendix G, Section G.2.) This means that the simulation does not guarantee results for non-penetrated holes. As the level of drill bit wear increases, the total drilling thrust force increases, and in accordance with the wear/no-wear weight assignation, the shear thrust force decreases and the ploughing force increases.

With an increased level of wear and the corresponding increased ploughing force, the drill bit was not able to completely push through the material, generating partially penetrated holes with high burrs. The simulation assumed the hole was penetrated every time and calculated the burr height and thickness value accordingly, which is a limitation of this model. Future studies should consider non-penetrated holes in modeling and experimentation. 


\subsubsection{Thrust Force Weight Designation}

An additional possible contribution to the disparity between the experimental and calculated results is the dependence of the sharp-dull force ratio on the drill bit geometry. The current weights were assigned based on the percentage of drill bit cutting edge radius wear. If, for example, the drill bit is $25 \%$ worn, then the total thrust force constitutes $75 \%$ shear drilling thrust force and $25 \%$ ploughing thrust force. Wear separation was present, but actual force separation may not be congruous with the level of cutting edge wear. The present sharp-dull drilling thrust force model having weights proportional to the drill bit cutting edge wear radius is a reasonable approach. An experimental assessment of sharp versus dull thrust forces present in relation to the level of wear of the drill bit cutting edge, together with the assignment of weights for the sharp-dull drilling thrust forces, could fine tune the calculated results and bring them closer to the experimental results.

\subsubsection{Simulation Burr Expectations}

When calculating simulation values for burr heights and thicknesses, the simulation assumes that when the drill bit penetrates the material and creates the hole and the burr, the resulting burr height and thickness will have a single uniform height and thickness value along the entire circumference of the hole. This value represents the average. An unvaried calculated value is bound to differ from a burr formed during an actual drilling experiment.

As discussed by Kim and Dornfeld [12], [26], and as demonstrated by the experiments described in this chapter, significant drill bit tool wear leads to material being ploughed beneath the drill instead of being sheared. Consequently, ploughing results in material fracturing in the centre of the region beneath the chisel edge and developing a crown burr, which is synonymous with burr irregularity. A less worn drill bit is generally expected to initiate material fracture at the outer cutting edge of the drill bit and generating a more uniform burr, often with a drill cap.

Given drill bit wear and expected uneven material fracture, it is not possible to deliver a $100 \%$ accurate prediction of how a burr will form, including maximum and minimum values in varying burr height and thickness. Material fracture during drilling would have to be monitored and analyzed live and a subsequent model developed for monitoring and predicting burr formation strictly based on material fracture from drilling. The above, therefore, explains another source of variability for burr formation as compared to the new model simulation results. 


\subsubsection{Material Differences}

Two aluminum alloys used in the experiment, AL 6061 T6 and AL 7075 T6, may contribute to the variability between simulated and experimental results.

The hardness of the two alloys differs, with 7075 being harder (Brinell 150) and 6061 being softer (Brinell 95). This difference could be a contributing factor to the differences in experimental results and the calculated values. The 7075 experimental values for $0 \%, 25 \%$, and $50 \%$ wear coincided with the simulation values more closely than the 6061 experimental values. 6061 is softer, so the burrs formed were more generous in height and thickness, thereby often exceeding the simulation prediction. This is reflected in Fig. 5.17, and 5.18 and in Tables G.1-G.6.

\subsubsection{Cutting Speed}

Cutting speed is not part of the burr formation model. The novel burr formation together with the tool wear model is designed with the primary shear zone of orthogonal cutting in mind. However, it does not account for cutting speed nor for cutting or friction forces. This could lead to differences in theoretical versus experimental results. In day-to-day drilling, friction in cutting is present, but for the purposes of this model it was not included because the model is focused on the primary shear zone, where friction forces are not present. (For additional details see Appendix A, Section A.4.)

\subsection{Conclusion}

This chapter presents a method for the validation of the new burr formation model by experimentation, which imitated day-to-day drilling conditions commonly encountered in aerospace manufacturing.

Artificial drill bit wear-down closely paralleled real life once drill bits were examined with an SEM; the cutting edge was reproduced in a 3D model and measured through manual on-screen image measurement. Average drill bit radius measurements were calculated from the measured values and fed into a Matlab simulation that calculated the burr heights and thicknesses based on the new burr formation model, as detailed in Section 5.5. The simulation calculated the theoretical values for each level of drill bit wear at feeds $.002, .004$, and $.006 \mathrm{in} / \mathrm{rev}$. The effectiveness of the simulation and drill bit measurement was further demonstrated experimentally by drilling 0.125" diameter holes in Al 6061 T6 and Al 7075 T6 plates of two thicknesses, .040" and .090", with each of the five drill bits at $0 \%, 25 \%$, $50 \%, 75 \%$, and $100 \%$ wear. 
As can be seen in the plots, the burr height and thickness results showed general agreement with the simulation outcomes for holes created with drill bits between 0\% and 50\%. For Al 6061 T6, at 75\% and $100 \%$ wear, where the ploughing mechanism was assumed to have been at work to a greater degree than cutting, the heights varied from the simulation results, on average, by a minimum of $713 \mu \mathrm{m}$. For $\mathrm{Al}$ 7075 T6 at $75 \%$ and $100 \%$ wear, the heights varied from the simulation results, on average, by a minimum of $709 \mu \mathrm{m}$. Burr thicknesses demonstrated a similar behaviour for both materials: good agreement with simulation values for the thicknesses from holes drilled by drill bits up to and including $50 \%$ wear, but acute differences were observable at $75 \%$ and $100 \%$ wear. This suggests that with a greater presence of the ploughing mechanism than shearing, burr heights and thicknesses increase dramatically. Furthermore, when ploughing was present in greater quantities than cutting because of greater wear, most holes were not completely penetrated, which resulted in greater thicknesses. The lack of a thickness that could be measured at the burr root led to the establishment of two types of thicknesses, a positive, $T_{1}$ and a negative, $T_{2} . T_{1}$ thicknesses enabled the measurement of thicknesses for holes that were drilled through. $T_{2}$ is a negative burr thickness, as it is in the direction opposite to that of a burr thickness for a completely penetrated hole.

There are several plausible reasons for the variance between simulation and experimental results: 1) mode of drill bit wear, 2) simulation burr height and thickness expectation versus real life burr height and thickness results with increased tool wear, 3) the model being appropriate for fully penetrated holes instead of those incompletely penetrated, 4) the method with which the sharp-dull thrust force weights were assigned, 5) hardness differences in the materials used, and 6) the model being designed for the primary shear zone, with the exclusion of the secondary shear zone.

In summary, the experiment was a successful evaluation of the new burr formation model, as it demonstrated that in-situ burr formation generally followed the created algorithm for burr height and thickness formation with gradually increasing degrees of drill bit wear. Moreover, the experiment was a suitably pragmatic methodology for burr growth prediction.

Furthermore, this model makes a direct link between the level of wear of the drill bit cutting edge and the presence of the ploughing force. Additionally, the model is shown to demonstrate closer alignment between simulation and calculated values for $0 \%$ to $50 \%$ wear than at $75 \%$ to $100 \%$ wear. Expanding this model to account for increased levels of wear, including those for non-penetrated holes, by utilizing ploughing thrust force measurement or ploughing thrust force extrapolation techniques as performed in [56], is a reasonable next step for this model. 
Given the work and experiments done in this dissertation, and the experimental data showing closer alignment between drill bit cutting edge wear for $0 \%$ to $50 \%$ wear versus the lack of alignment at $75 \%$ to $100 \%$ wear, there is room for further work to expand the model and adapt it for drill bits at $75 \%$ and $100 \%$ wear to ensure its versatility and improve its predictive abilities for levels of drill bit wear beyond $50 \%$. 


\section{CHAPTER 6 CONCLUSION}

In this dissertation, a methodology was developed for modeling burr formation with tool wear that incorporated varying wear conditions for a segmented drill bit cutting edge. The resulting burr height and thickness were obtained by establishing a new drilling thrust force, combining cutting and drilling with ploughing from tool wear. The proposed model allows for adjustment of the cutting and ploughing components by incorporating a wear/no-wear condition through a segmented cutting edge approach. The radius of the drill bit cutting edge, worn or sharp, is a key variable in the methodology together with the key factors of process parameters and material properties.

While multiple strategies for drilling burr formation modeling and tool wear modeling for ductile metals have been developed, the findings from this dissertation are novel since the new burr formation model addresses both burr height and thickness growth resulting from a combination of shearing and ploughing forces; this is the outcome of varying levels of wear of the drill bit cutting edge.

Previous methods reviewed in Chapter 2 address only models within the scope of either drilling burr growth, tool wear, or slip-line fields. For burr growth, the existing methods discussed both drilling parameters and drill bits as ideal without variance and with sharp cutting edges, without taking into account variance that stems from normal wear and tear.

\subsection{Summary of Proposed Mode and Research Conclusions}

The proposed model for burr formation with drilling was developed in two stages. As described in Chapter 3, the initial research effort focused on sensitivity matrix development, by adapting a burr height and thickness model for sharp tools from Kim and Dornfeld [1] (see Appendix A). The goal of the initial research described in Chapter 3, Section 3.7 was to formulate a burr sensitivity model that defined settings for feeds and point angles in real-life aerospace manufacturing drilling applications of ductile metals that produce minimal burrs. A Jacobian transformation was applied to calculate the best nonlinear approximation near a given point to the differentiable functions of burr height and thickness; a sensitivity methodology was subsequently formed. The method was evaluated through a simulation, and expected trends of burr height and thickness growth and their rates of change emerged with respect to feed and point angle ranges. The expected parameter sets that could provide the smallest burr height, thickness, and rates of change were not clearly apparent. Optimization was employed to 
obtain feeds and point angles that produced a minimal burr with the lowest change in growth, incorporating burr height, thickness, and their rates of change together with hyperparameters of weights and efficiencies. Incorporating efficiencies was the first step in examining the effects of realistic drilling conditions. The calculated burr growth trend observed, which could stem from point angle changes, was an indicator that tool wear may be a contributor to burr growth. This is inferred, since the point angle was treated as a continuous variable in the burr sensitivity model together with burr growth and rate of change of burr growth increasing with an increased point angle. These findings suggested that tool wear should be examined further.

Chapter 4 describes the adaptation of the ploughing tool wear model by Waldorf, DeVor and Kapoor [2] and the development of the new burr formation model, which is the focus of this dissertation. The sharp drilling burr formation model was merged with the ploughing model for orthogonal cutting with a worn cutting edge. At the core of the new methodology is a novel thrust force, merging a drilling thrust force where a shear force acts to shear the material, with a ploughing thrust force that pushes the material with a rounded edge. The proposed model incorporates the changed drill bit cutting edge radius from tool wear together with a segmented cutting edge approach, enabling an elemental thrust force to act on individual cutting edge segments. Realistic manufacturing floor conditions of varying levels of drill bit wear are reflected in the weighted approach, where a level of wear can be assigned to each drill bit cutting edge segment. The new thrust force led to a new practical burr height and thickness model that diverged from previous ideal-condition methodologies. The model was evaluated first with a simulation, by calculating burr heights and thicknesses using five drill bits at various levels of wear, ranging from $0 \%$ to $100 \%,{ }^{4}$ two aluminum alloys commonly used in aerospace manufacturing, Al 6061 T6 and Al 7075 T6, and three different feeds. The results showed an expected trend: with an increased level of drill bit wear burr heights and thicknesses were progressively greater. The simulation results were promising, as the new burr growth model showed an assumed trend. Experimental evaluation was the next step.

Chapter 5 details the experimental method for the new burr formation model, using drilling tool and process conditions employed in the simulation. The materials, drill bit wear and process parameters employed both in the simulation and the experiment were set up to resemble a real-life drilling

\footnotetext{
${ }^{4}$ As established in Chapter 5, Section 5.1.4, the target drill bit wear levels were $0 \%, 25 \%, 50 \%, 75 \%$, and $100 \%$. In non-ideal conditions they were $4.61 \%, 24.31 \%, 49.1 \%, 76.6 \%$, and $100 \%$. For the purposes of this dissertation, when discussing target $0 \%, 25 \%, 50 \%, 75 \%$, and $100 \%$ wear, the reference implies calculations using actual drill bit wear levels.
} 
environment. Aluminum sheet metal of two different thicknesses was drilled with the five drill bits with varying degrees of wear, at three different feeds; burr heights and thicknesses were measured with an SEM. A comparison was then made between sets of experimental and simulation data. The comparisons showed that

- The model successfully estimated burr height and thickness trends with increasing levels of tool wear, for penetrated holes, as demonstrated by the data;

- Material thickness was not a factor in burr growth; and,

- The total thrust force and initial thickness of deforming ductile materials can adequately predict calculated burr height and thickness growth for drilling with $0 \%$ to $50 \%$ worn drill bits.

Although the new burr formation model achieved burr height and thickness prediction trends, divergences were observed.

Calculated values for burr heights and thicknesses assumed that the resulting burr height and thickness would have a uniform height and thickness value along the entire circumference of the hole. Simulation values, in reality, represented the average predicted height and thickness. An accurate prediction of how burr height and thickness minima and maxima will form is unlikely for the conditions of using a worn drill bit, coupled with expected uneven material fracture at drilling initiation. Additional experimentation dedicated to analyzing material rupture patterns in drilling would be required.

Experimental burr heights and thicknesses were close in proximity to calculated values at $0 \%$ to $50 \%$ levels of wear, while at $75 \%$ and $100 \%$ levels of wear measured burr height and thickness values differed by $710 \mu \mathrm{m}$ or greater. This disparity was observed exclusively in non-penetrated holes. The calculated model could not be relied upon to deliver results close to experimental values for non-penetrated holes as the burr formation model assumes that every hole is penetrated.

The sharp-dull force ratios in the novel wear/no-wear model were designed to depend only on those assumed ratios. The disparity between calculated and experimental burr results may be the absence of the force ratio. An assessment of the sharp to dull force split ratio could bring the predicted values closer to experimental results.

Differences in material hardness between Al 6061 T6 and Al 7075 T6 are another likely contributor to simulated versus measured results. Al 6061 T6 is softer than Al 7075 T6, which resulted in its drilled burrs being more substantial in height and thickness. There were considerable differences between the 
calculated and experimental values for burrs resulting from Al 6061 T6, at some points diverging more than the burr heights and thicknesses obtained from drilling Al 7075 T6.

The new burr formation model does not account for the secondary shear zone. Therefore, the cutting speed, the cutting force, and the friction force are excluded. The model is designed for the primary shear zone. This also may have contributed to differences between predicted and measured results.

\subsection{Contributions}

Contributions explaining the differences between calculated and actual results can lead to further exploration in future research.

The methods and results presented in this dissertation show good promise for understanding and quantifying the effects of tool wear on drilling burr growth, making the following contributions:

- A better understanding of the mechanisms that contribute to burr formation;

- A greater comprehension of how tool wear impacts burr height and thickness growth;

- A burr height and thickness model that adequately predicts burr growth for $0 \%$ to $50 \%$ drill bit wear; and

- A novel method that can be expanded to address multiple degrees of drill bit wear for other materials and drill bit geometries.

The balance of this chapter is devoted to topics recommended for future study by the manufacturing engineering community.

\subsection{Recommendations for Future Work}

The new burr formation model warrants further exploration. Some of the parameter values of the proposed model need to be considered in more detail and expanded on through several avenues.

As mentioned, the present burr formation model delivers an average uniform burr height and thickness. Drilling with a worn bit involves the material fracturing in the centre of the region beneath the chisel edge and developing a crown burr. Material fracture during drilling would have to be monitored live to collect data on material behaviour, thrust force variation, and the range of resulting burr heights and thicknesses. This would allow for the development of a fracture-to-burr material model. Given that the 
simulation indicated that the current model is more suited for penetrated holes than non-penetrated ones, such a model could be further modified to forecast burr growth behaviour when hole penetration is not expected.

Another augmentation of the burr formation model could involve scrutinizing the split between sharp and dull force ratios and assigning weights accordingly. Currently, the ratio of thrust-to-plough force ratio is quantified via drill bit wear radius increase. Studying the shear-ploughing force proportion and revising the weight assignation with a different assigned force ratio might adjust the simulation result. Calculated versus experimental burr values could be revisited to assess if the disparity between the two groups is minimized.

The new burr formation model could be supplemented by accounting for the secondary shear zone, and in that way incorporate the cutting speed and friction force. As previously stated, the model is presently designed for the primary shear zone, which plausibly could have contributed to the discrepancy between predicted and measured results.

The model was able to predict a relationship between simulation and experimental data between $0 \%$ and $50 \%$, though more data would be desirable. Moreover, a larger sample size could be used to test the force model for a range of materials. Al 6061 T6 and 7075 T6 are standard, commonly used ductile materials. Given the increased use of superalloys in the aerospace and automotive industries, for example, Inconel alloys, it would be of value to test their behaviour and corresponding burr growth with the proposed model. Aluminum-lithium alloys, used extensively in the aerospace industry, given their weight-savings advantages, are another alloy family worth exploring. Advanced composite materials would also be worth exploring because of their broadening application in aircraft manufacturing.

The extension of the proposed model to new materials, especially more exotic ones that are used in aerospace, would introduce the re-examination of the shear strength and strain hardening relationships at the core of the burr formation model. The stress-strain curves of Inconel and advanced composites diverge significantly from the standard aerospace-grade aluminum alloy. The proposed methodology was designed for ductile materials and a perfectly plastic material model. The burr formation model itself would likely require modification in both cases to adjust for the different shear strength and strain hardening behaviours.

In the consideration of new materials, the exploration of different drill bit geometries and, therefore, modification of the proposed model to accommodate the diversity, would be a natural progression. In 
its design, the new model utilized the geometry of a conventional point twist drill, the most common tip style with a segmented drill bit cutting edge approach. The proposed model could be adapted to other drill classifications. The exploration of drill types could begin with the family of twist drills, one of the most commonly used drills, providing a large variety to analyze. Counterbore and countersink drill bits could be other groups to consider. The burr growth model would have to be adjusted each time. Additional studies would have to be completed to learn the unique wear mechanisms experienced by the distinctive drill bit geometries and then incorporate the additional cutting edge radii or surfaces that are most susceptible to wear and practical to measure. The next step, taking superalloys and advanced composites into account, would be to assess the tools required to drill them. Inconel's toughness makes it challenging to work with, and as such, standard twist point HSS drill bits would not be useful. Drill bits suitable for Inconel could also lead to further modifications to the new burr growth model because their material may become a significant factor. Advanced composites are drilled with their own class of drill bits that, like Inconel, would require additional investigation with respect to the tool wear patterns, that could then be incorporated into the burr growth model.

Another possible direction of future work is further study of the worn drill bit cutting edge. In the burr growth model, the drill bits were worn down artificially in order to test burr growth at varied and distinct levels of wear. Neither the volume of sheet metal drilled with them nor the method presented challenging drilling conditions. The variation of the cutting edge radius with time and part volume needs to be explored. Experiments of longer duration could be run in which the drill bit cutting edge is measured following varying amounts of drilling, and the burr formation model could be expanded by incorporating how the wear radius changes over time. The new drilling thrust force for which the edge radius is an input could become a time-varying function as a result. The outcome could lead to an even more accurate burr growth model, providing further insight into burr growth with respect to time, force, and wear variation.

The burr growth model developed in this dissertation provides the beginning of an understanding of how wear affects burr growth. Under dynamic conditions of progressive wear, the thrust force gradually shifts from shearing to ploughing, impacting chip size and therefore burr size. The proposed burr height and thickness model tends to predict in accordance with observation, meaning that a larger cutting edge radius applied to a greater number of segments of the drill bit cutting edge would result in a greater burr height and thickness. With this in mind, utilizing the results of the burr height and thickness growth methodology to provide information regarding advancement in burr minimization could be a fruitful 
direction for future research. This could would allow for the burr growth model to serve as a guideline for improvement of both drilling and tool monitoring practices in aerospace manufacturing, with prospects of expanding the methodology to other industries. 


\section{APPENDIX A: DRILLING BURR FORMATION MODEL FOR SHARP TOOLS AND ORTHOGONAL CUTTING DESCRIPTION}

\section{A.1 Work of the Drilling Thrust Force $W_{T H}$}

To determine $\Delta W_{T H}$, the fluctuation of the thrust force as the material was being drilled was examined by Kim and Dornfeld [12]. The tests consisted of using a split-point twist drill, $3.175 \mathrm{~mm}$ in diameter, to drill stainless steel AISI $304 \mathrm{~L}$ at a feed of $0.0317 \mathrm{~mm} / \mathrm{rev}$. The thrust force during the drilling process was measured using a dynamometer.

The thrust force was measured from the period of initial material deformation to the point in time that the drill's outer cutting edge lined up with the newly drilled hole edge, as per the drilling phases illustrated in Fig. 3.1. Throughout the steady-state drilling, a constant thrust force was observed, followed by a decrease in this force once material deformation was initiated, [12].

The outcome of these observations became the work of the drilling thrust force in a quadratic equation is presented in eq. (A.1) [12, eq. (5)]:

$$
\begin{gathered}
\Delta \mathrm{W}_{\mathrm{TH}}=F_{T H} \int_{0}^{L}\left(1-\frac{y^{2}}{\left(t_{o}+\frac{R}{\tan p}\right)^{2}}\right) d y \\
\left(t_{o}+\frac{R}{\tan p}\right)=L
\end{gathered}
$$

where $F_{T H}$ is the constant thrust force, $y$ is the distance of drill advancement into the drilled material, $R$ is the drill radius, $p$ is the drill half point angle, and $t_{o}$ is the initial chip thickness.

Once integrated with the distance from 0 to $L, \Delta W_{T H}$ becomes eq. (A.2), as derived from [12, eq. (15)]:

$$
\Delta \mathrm{W}_{\mathrm{TH}}=\frac{2}{3} F_{t h} L
$$

Kim and Dornfeld's burr formation model [12] is based on orthogonal cutting and the Merchant model, illustrated in Fig. 3.2. For details on orthogonal cutting and Merchant models see Section A.4. 

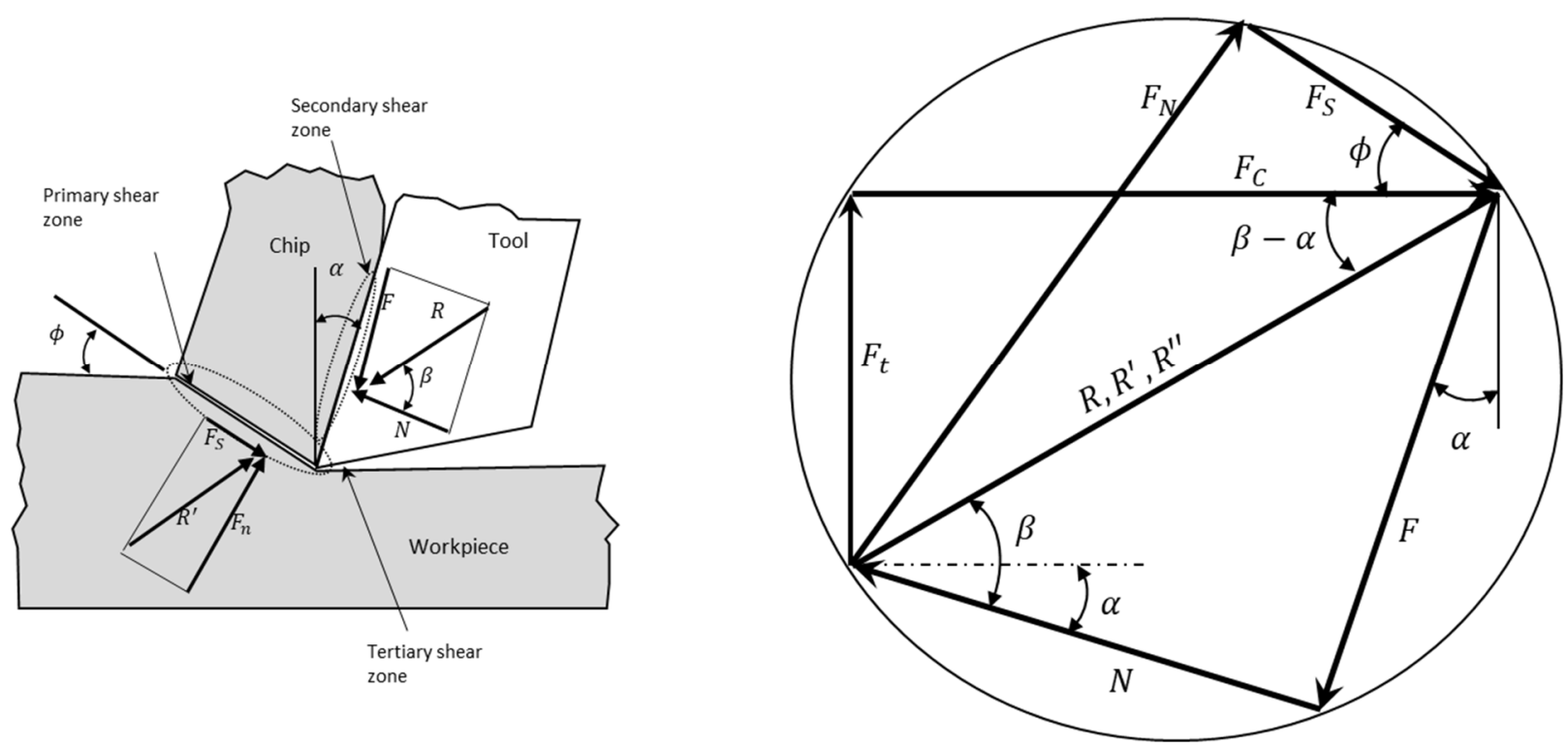

Fig.3.2 Orthogonal cutting model and Merchant circle model

The fundamental equation for thrust force in orthogonal cutting is, [31]:

$$
\Delta F_{t h}=\frac{F_{S} \sin \left(\beta-\alpha_{n}\right)}{\cos \left(\phi+\beta-\alpha_{n}\right)}
$$

where $F_{S}$ is the shear force, $\beta$ is the friction angle, $\alpha_{n}$ is the normal rake angle, and $\phi$ is the shear angle.

The shear force is further broken down as follows, [31]:

$$
F_{S}=A_{S} \tau_{S}
$$

where $A_{S}$ is the shear plane area and $\tau_{S}$ is the shear strength of the workpiece.

For drilling, the shear plane area is expressed as the width of cut, [31]:

$$
A_{S}=\frac{f \Delta w}{\sin \phi}
$$

where $f$ is the feed and $\Delta w=\left(r_{i+1-} r_{i}\right)$ is the width of the ith segment along the segmented drill bit cutting edge.

Kim and Dornfeld [12] define $\rho$ as the relative radius:

$$
\rho=\frac{r}{R}
$$

where $r$ is the distance from the drill bit centreline to each segment on the sectioned drill bit cutting edge and $\mathrm{R}$ is the drill bit radius. 
Consequently $\Delta w$ is updated as follows:

$$
\Delta w_{i}=R\left(\rho_{i+1-} \rho_{i}\right)
$$

The updated thrust force acting on each segment along the drill cutting edge is expanded to:

$$
\Delta F_{t h}=\frac{f R\left(\rho_{i+1-} \rho_{i}\right) \tau_{s} \sin \left(\beta-\alpha_{n}\right)}{\sin \phi \cos \left(\phi+\beta-\alpha_{n}\right)}
$$

The thrust force for drilling initially only incorporated a normal rake angle, $\alpha_{n}$, across the main cutting edge, where $\delta_{0}$ is the helix angle in a two-fluted twist drill [12, eq. (11)]:

$$
\alpha_{n}=\tan ^{-1}\left\{\frac{\tan \delta_{0}\left(\rho^{2}-w^{2} \sin ^{2} p\right)-w \sin p \cos p}{\sin p\left(\rho^{2}-w^{2}\right)^{\frac{1}{2}}}\right\}
$$

The thrust force was further modified by the incorporation of a dynamic rake angle $\alpha_{d}$, as illustrated in Fig. A.1.

$\alpha_{d}$ accounts for the cutting edge moving in a circular motion at an angle oblique to the direction of the tool velocity, the drilling feed, rotational motion, and, $v_{c}$, the varying cutting velocity along the drill bit cutting edge. $r_{i}$ in Fig. A.1 is the distance from the centre of the drill to each element, with $i$ representing the individual element along the cutting edge and $\gamma_{c}$ representing the quantity $f / 2 \pi r_{i}$.

In drilling, $v_{c}$ represents the varying cutting velocity, which is the resultant of feed in the downward direction and tangential velocity, $v_{\theta}=\omega r$, where $\omega$ is the angular velocity and $r$ is the distance from the centre of rotation. When utilizing drill bit cutting edge segmentation, the further a particular segment is from the center of the drill, the closer the cutting mechanism resembles orthogonal cutting. The closer the segment being considered is to the drill bit center, the greater the $\alpha_{n}$. The dynamic rake angle $\alpha_{d}$ is therefore composed of $\alpha_{n}$ and $\gamma_{c}$, [12, eq. (12)]. $\gamma_{c}$ is the angle with respect to which the rake angle changes, depending on the location of the segment being considered, along the drill bit cutting edge. 


$$
\alpha_{d}=\alpha_{n}+\tan ^{-1}\left(\frac{f}{2 \pi r_{i}}\right)
$$

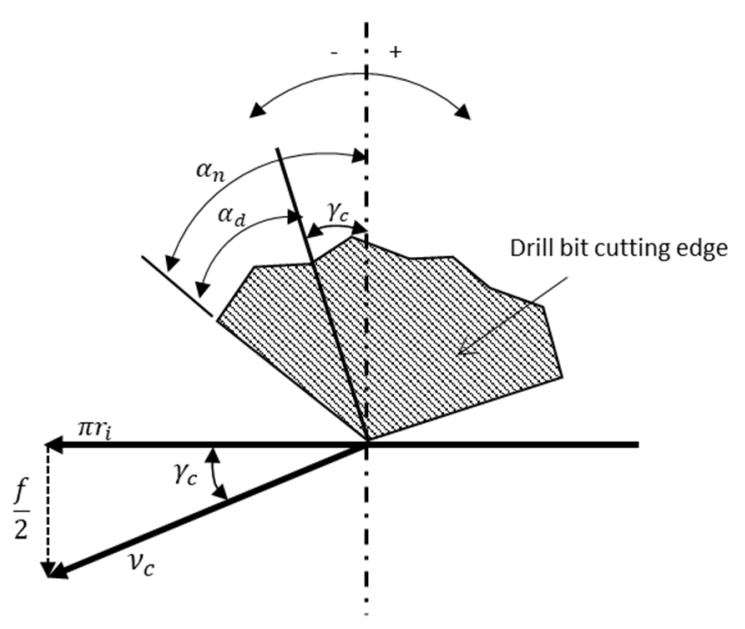

Fig. A.1 Dynamic rake angle, adapted from [12, Fig. 5]

In order to complete the thrust force, further assumptions and modifications were made with respect to the shear strength, $\tau_{s}$, to complete the burr formation model. In general, material shear strength and tensile strength $\sigma_{u}$ have similar strain hardening behaviors, so the following relationship holds, as derived from [12, eq. (14)]:

$$
\tau_{s}=\frac{\sqrt{2}}{3} \sigma_{u}
$$

The substitution of these relations and summation of these thrust forces enabled the establishment of the total drilling thrust force, shown in eq. (3.6), derived from [12, eq. (15)]:

$$
F_{T H}=\frac{\sqrt{2}}{3} R f \cdot \sigma_{u} \cdot \sum_{i=1}^{N}\left\{\frac{\sin \left(\frac{\pi}{6}-\frac{\alpha_{d}}{2}\right)\left(\rho_{i+1-} \rho_{i}\right)}{\sin \left(\frac{\pi}{6}+\frac{\alpha_{d}}{4}\right) \cos \left(\frac{\pi}{3}-\frac{\alpha_{d}}{4}\right)}\right\}
$$

Reiterating the work of the $\Delta W_{\mathrm{TH}}$, eq. (A.2) derived from [12, eqs. (3), (15)]:

$$
\Delta \mathrm{W}_{\mathrm{TH}}=\frac{2}{3} F_{t h} L
$$


$\Delta W_{\mathrm{TH}}$ expands to eq. (3.4) [12, eqs. (3), (15)], discussed in Chapter 3, Section 3.2:

$$
\Delta W_{\mathrm{TH}}=\frac{2}{3}\left[\frac{\sqrt{2}}{3} R f \cdot \sigma_{u} \cdot \sum_{i=1}^{N}\left\{\frac{\sin \left(\frac{\pi}{6}-\frac{\alpha_{d}}{2}\right)\left(\rho_{i+1-} \rho_{i}\right)}{\sin \left(\frac{\pi}{6}+\frac{\alpha_{d}}{4}\right) \cos \left(\frac{\pi}{3}-\frac{\alpha_{d}}{4}\right)}\right\}\right] L
$$

\section{A.2 Work of Material Deformation $W_{D F}$}

As described in Section 3.1, work of material deformation, $\Delta W_{D F}$, is obtained through modeling the drilled material as a thin plate, circular in shape, which transforms into a cone resulting from material bending and elongation. The detailed geometry of this transformation process is represented in Fig. 3.5[12, Fig. 6], illustrated as a series of bending elongation steps as the shape translates from abde to fdhg.

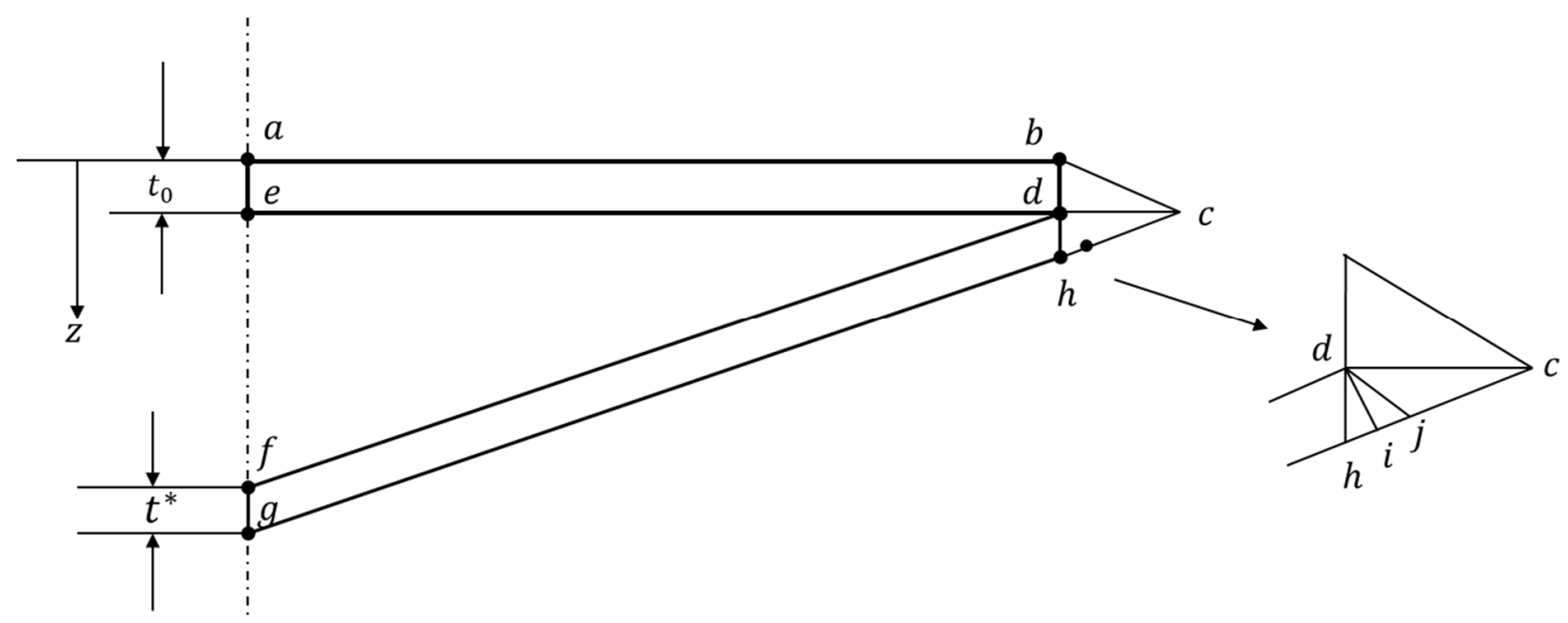

Fig. 3.5 Half cross-section of material prior to and after deformation, adapted from [12, Fig. 6]

Deformation initiates with material elongation where points abde translate their position to points $f d h g$. Next, the material bends along segment $\bar{d} \bar{b}$, and finally it bends along segment $\overline{d c}$.

$W_{D F}$ is the sum of the work of elongation and bending steps, illustrated by $\Delta W_{I}, \Delta W_{I I}$, and $\Delta W_{I I I}$.

Shear strain is a component of the deformation. Its effects are limited to a very small region around the drill centre and, therefore, the work performed by the shear strain is negligible. 
To find $\Delta W_{I}$, strain distribution along $\bar{e} \bar{d}$ was used, and work for material elongation was finalized to be eq. (A.13) [12, eq. (21)]:

$$
\Delta W_{I}=\frac{3}{4} \pi R^{2} t_{0} \sigma_{y} \sin p\left\{\ln \left(\frac{1}{\sin p}\right)+\frac{t_{0}}{R} \sin ^{2} p \cos p\right\}
$$

Work for material bending deformation along $\overline{b d}$ is described by eq. (A.14) [12, eq. (22)]:

$$
\Delta W_{I I}=M_{b} \cdot \Delta \theta=\frac{1}{2} \pi \sigma_{y}\left(\frac{\pi}{2}-p\right) R t_{0}^{2}
$$

where $M_{b}$ is the bending moment assuming perfect plastic material behaviour and $\Delta \theta$ is the angle of rotation of the bending deformation.

$\Delta W_{I I I}$, work for material bending along $\overline{d c}$, is negligible compared to the other terms because in the development of this model, drill bit radius $R$ is approximately 50 times bigger than the initial deformed material thickness $t_{0}$.

The work for material deformation is, therefore, the sum of $\Delta W_{I}$ and $\Delta W_{I I}$, shown in eq. (3.6) [12, eq. (24)], discussed in Chapter 3, Section 3.3:

$$
\begin{aligned}
\Delta W_{D F}=\Delta W_{I}+ & \Delta W_{I I} \\
& =\frac{3}{4} \pi R^{2} t_{0} \sigma_{y} \sin p\left\{\ln \left(\frac{1}{\sin p}\right)+\frac{t_{0}}{R} \sin ^{2} p \cos p\right\} \\
& +\frac{1}{2} \pi \sigma_{y}\left(\frac{\pi}{2}-p\right) R t_{0}^{2}
\end{aligned}
$$

\section{A.3 Final Burr Formation}

Total work is the outcome of the steady-state thrust force and the distance from the deformation initiation point (see phase 1 in Fig. 3.1) to the stage where the outer drill cutting edge is in line with the exit surface (see phase 3 in Fig. 3.1). The sum of these two work components forms a quadratic equation, the solution of which is the initial chip thickness of deforming material, $t_{0}$. The steps explaining how the equation is formed and solved are shown as follows with eqs. (A.15), (A.16), derived from [12, eqs. (1), (24)]: 


$$
\begin{gathered}
\Delta W_{T O T A L}=F_{t h}\left(t_{o}+\frac{R}{\tan p}\right)=F_{t h} L \\
\Delta W_{T O T A L}=\Delta W_{D F}+\Delta \mathrm{W}_{\mathrm{TH}}=F_{t h} L
\end{gathered}
$$

The resulting total work equation $\Delta W_{\text {TOTAL }}$ is eq. (3.6), ,[12, eq. (25)], discussed in Chapter 3, Section 3.4:

$$
\begin{aligned}
\frac{3}{4} \pi R^{2} t_{0} \sigma_{y} \sin p & \left\{\ln \left(\frac{1}{\sin p}\right)+\frac{t_{0}}{R} \sin ^{2} p \cos p\right\}+\frac{1}{2} \pi \sigma_{y}\left(\frac{\pi}{2}-p\right) R t_{0}^{2}+\frac{2}{3} F_{t h} L=F_{t h} L \\
= & W_{\text {TOTAL }}
\end{aligned}
$$


A.3.1 Burr Formation Model for Sharp Tools Flowchart

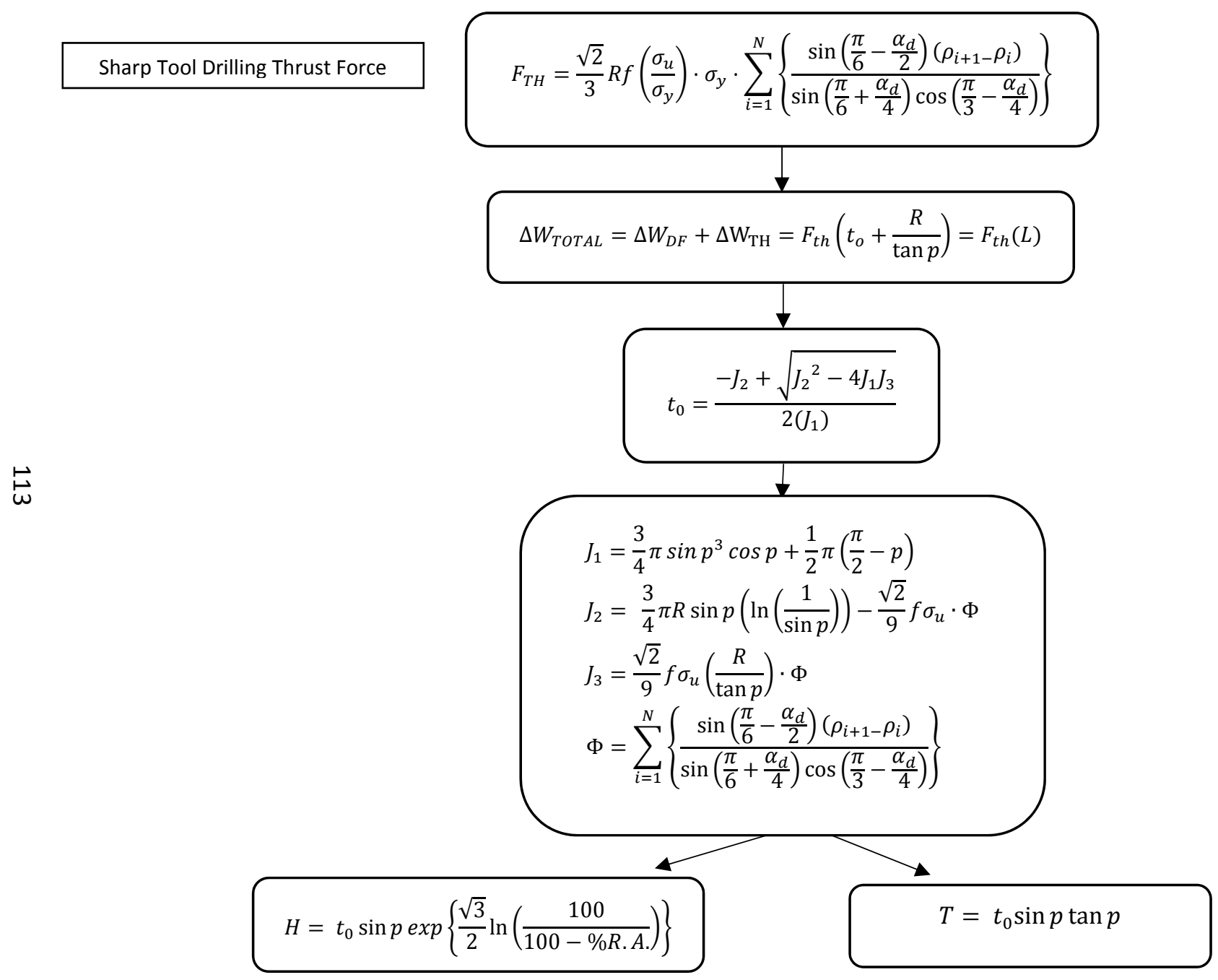

Fig. A.2 Burr Formation Model for Sharp Tools Flowchart 


\section{A.4 Orthogonal Cutting and Merchant Models}

\section{A.4.1 Orthogonal Cutting Model}

According to [31] and [34], material removal in drilling is based on orthogonal cutting, in which work material is removed by a cutting edge of a straight tool perpendicular to the direction of relative toolworkpiece motion. This is also known as the cutting velocity. The cutting edge is assumed to be sharp without a radius. The orthogonal cutting mechanism shears a chip from the work.

The orientations of uncut chip thickness and velocity determine the direction the cutting forces will be applied. Moreover, in orthogonal cutting there are three deformation zones: primary, secondary, and tertiary. According to [31] and [34], a chip is formed when the cutting edge of the tool makes contact with the workpiece and is sheared. This shearing occurs in the primary shear zone. After the chip is formed, friction between the tool and the chip results in the secondary shear, which occurs in the secondary shear zone, as shown in Fig 3.2. The tertiary zone is where the tool flank scrapes against the freshly cut work surface [31], [34]. This dissertation research focuses specifically on the primary shear zone, as does the Merchant model which is expanded upon in the subsequent paragraphs.

\section{A.4.1.1 Forces in Orthogonal Cutting}

In orthogonal cutting there are a number of forces acting on the chip, as depicted in Fig. 3.2. The three forces used in the Merchant model, which is significant for this research, include the cutting force, shear force, and thrust force.

As shown in Fig. 3.2, $F$ is the friction force between the tool and the chip, $N$ is the force normal to $F$. To form the resultant force $R, F$ and $N$ are added vectorially. $R$ is, in turn, oriented at friction angle $\beta . F_{S}$ is the force causing shear deformation in the shear plane. The force normal to the shear force is $F_{N}$, the normal force. $F_{S}$ and $F_{N}$ are forces applied on the chip by the workpiece. $F_{S}$ and $F_{N}$ added vectorially result in force $R^{\prime}$, which is opposite in direction and equal in magnitude to $R$, as can be seen in Fig 3.2.

The shear stress $S$ acts along the shear plane, which is the plane between the chip and the workpiece. Shear stress is the level of stress necessary for material removal.

$$
S=\frac{F_{S}}{A_{s}}
$$

$A_{s}$ is the shear plane area, as initially shown in Section A.1, and is calculated as follows: 


$$
A_{s}=\frac{t_{0} w}{\sin \phi}
$$

According to [31], $F_{c}$ is the force in the direction of cutting, which is the same direction as the cutting speed, $v . F_{t}$, the thrust force, is perpendicular to the cutting force $F_{c}$, in the direction of the feed. Their resultant force is $R^{\prime \prime}$.

Friction, normal, and shear forces can be calculated if cutting and thrust forces are known.

\section{A.4.2 Merchant Model}

Eugene Merchant derived the Merchant equation, which is rooted in orthogonal cutting, as described in [31], [34]. Merchant's model is focused on analyzing the primary shear zone and suggests that the shear zone is also a thin plane.

In Merchant's model, shear stress is defined through the relationship between the cutting force $F_{c}$ and thrust force $F_{t}$, together with the shear angle $\phi$, initial chip thickness $t_{0}$, and chip width $w$, starting with $F_{S}$, derived from the force diagram in Fig. 3.3:

$$
F_{S}=F_{c} \cos \phi-F_{t} \sin \phi
$$

Incorporating $S$ and $A_{S}$, arriving at the following:

$$
\tau=\frac{F_{c} \cos \phi-F_{t} \sin \phi}{\left(t_{0} w / \sin \phi\right)}
$$

The shear angle $\phi$ is assumed to be the main angle from the tool cutting edge that results in shear deformation and, consequently, chip formation. Shear deformation takes place at $\phi$ because the work material's shear strength is equal to shear stress at $\phi$. No other shear angle can lead to chip formation because in other shear angles, shear strength is greater than the shear stress.

According to [31], Merchant theorized that during orthogonal cutting the path of minimum energy will be favoured by the work material. Consequently, the work material will choose the shear plane and shear angle that correspond to minimal energy during metal cutting. Through a derivative of eq. (A. 15) with respect to $\phi$, the Merchant equation is obtained:

$$
\phi=45+\frac{\alpha}{2}-\frac{\beta}{2}
$$


The significance of Merchant's equation is its ability to define the correlation between friction angle $\beta$ (also referred to as tool-chip friction), the shear plane angle, and the rake angle. The relationship states that an increase in the shear plane angle $\phi$ results in a greater rake angle $\alpha$ and decreases the friction angle $\beta$. When applying Merchant's equation, certain premises must be accounted for, including temperature and strain rate, which have no impact on the work material's shear strength. The equation, therefore, must be regarded as interpolative instead of exact, because in daily machining operations both temperature and strain rate are taken into consideration. 


\section{APPENDIX B: BURR FORMATION MODEL PLOTS}

\section{B.1 Burr Heights and Thicknesses with Respect to a Range of Feeds}
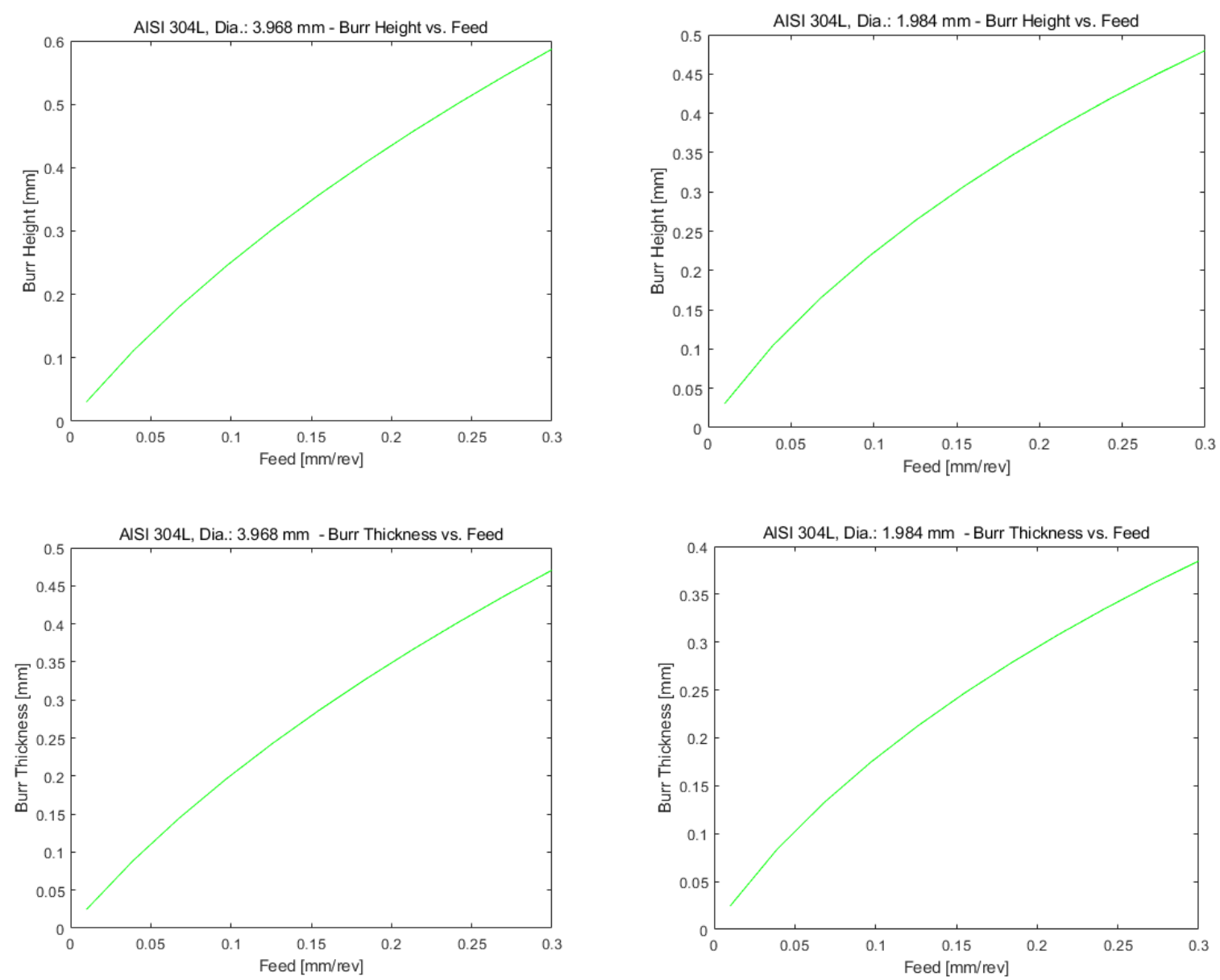

Fig. B.1 Burr heights and thicknesses vs. feed from AISI 304L 

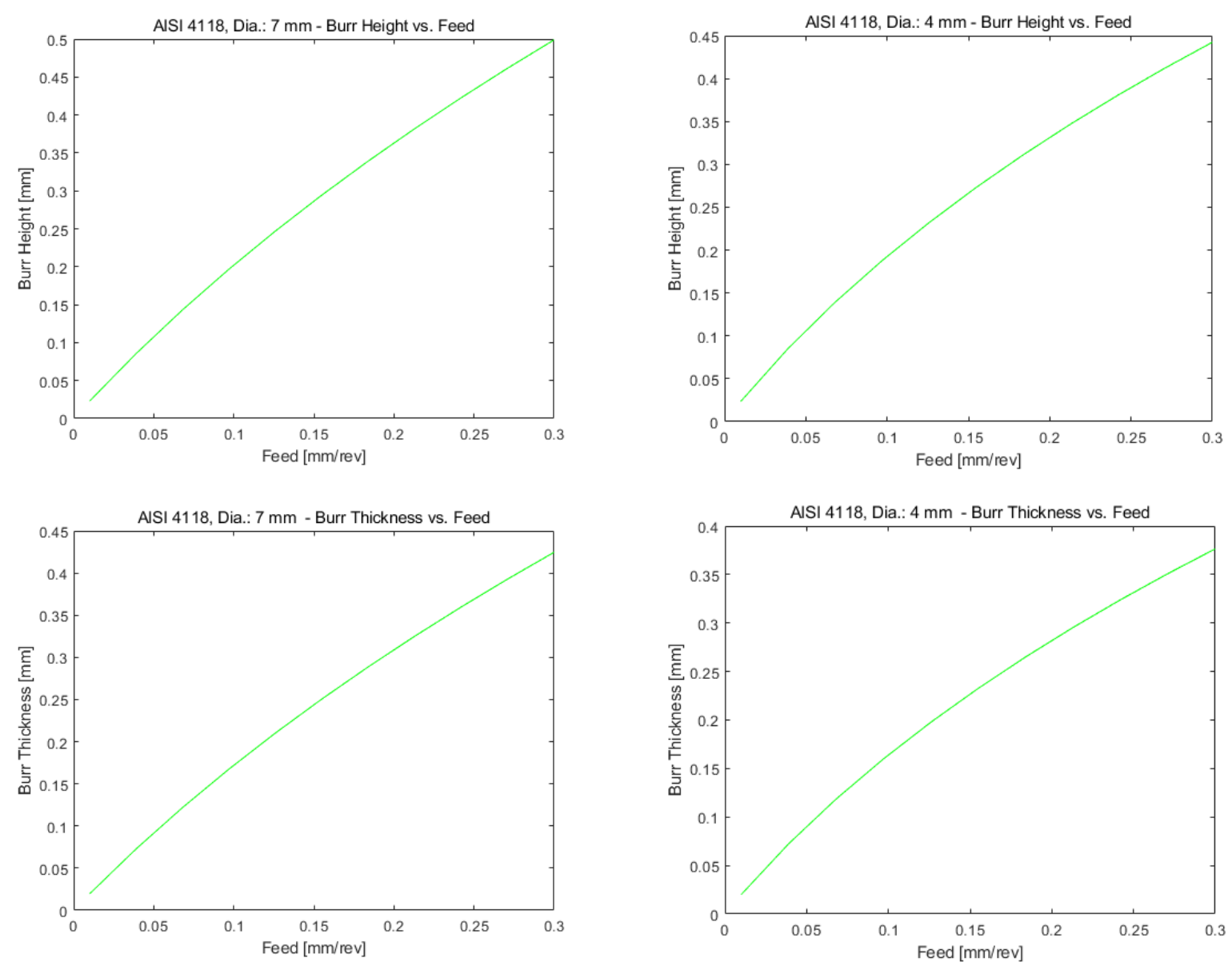

Fig. B.2 Burr heights and thicknesses vs. feed from AISI 4118 


\section{B.2 Burr Heights and Thicknesses with Respect to a Range of Point Angles}
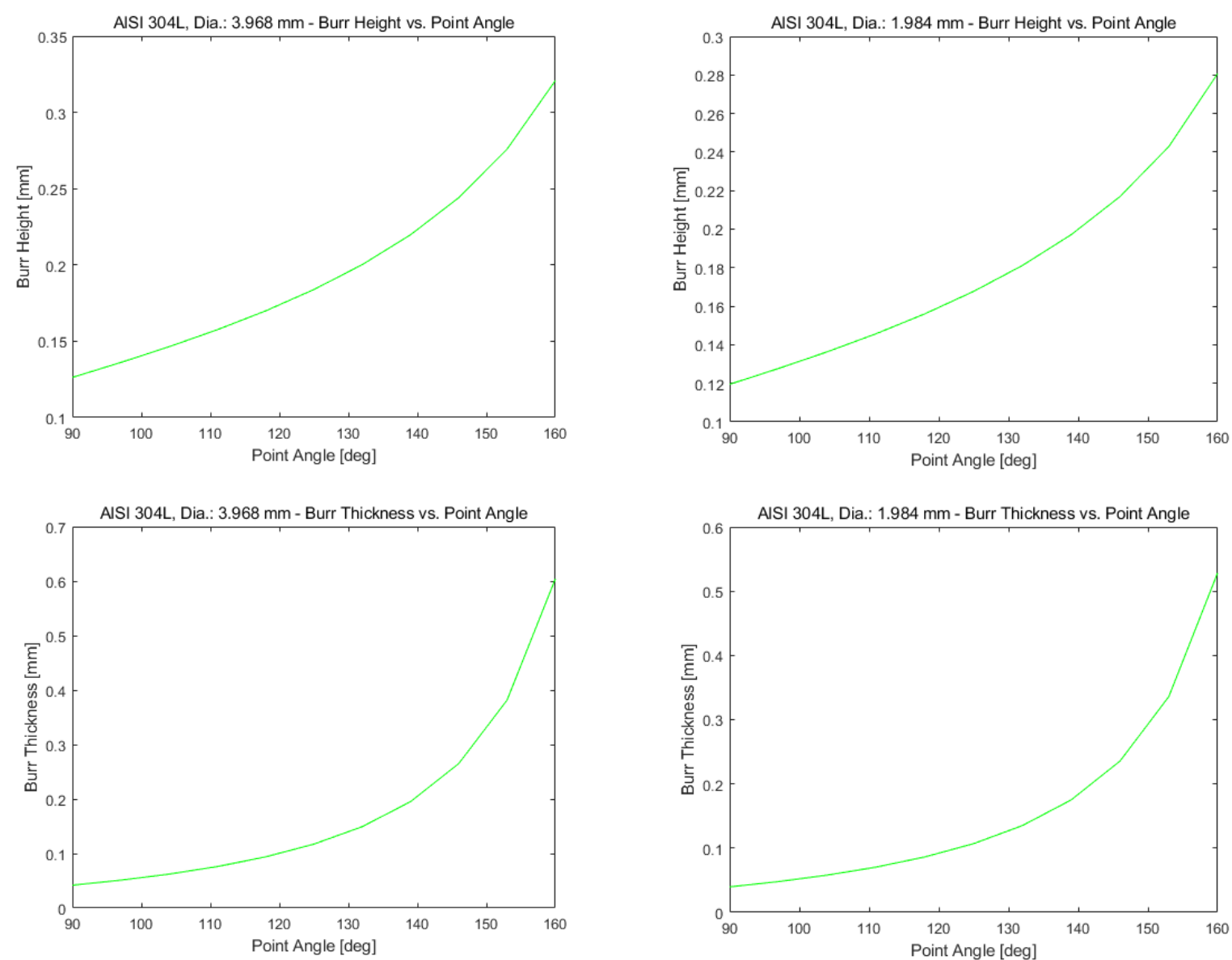

Fig. B.3 Burr heights and thicknesses vs. point angle from AISI 304L 

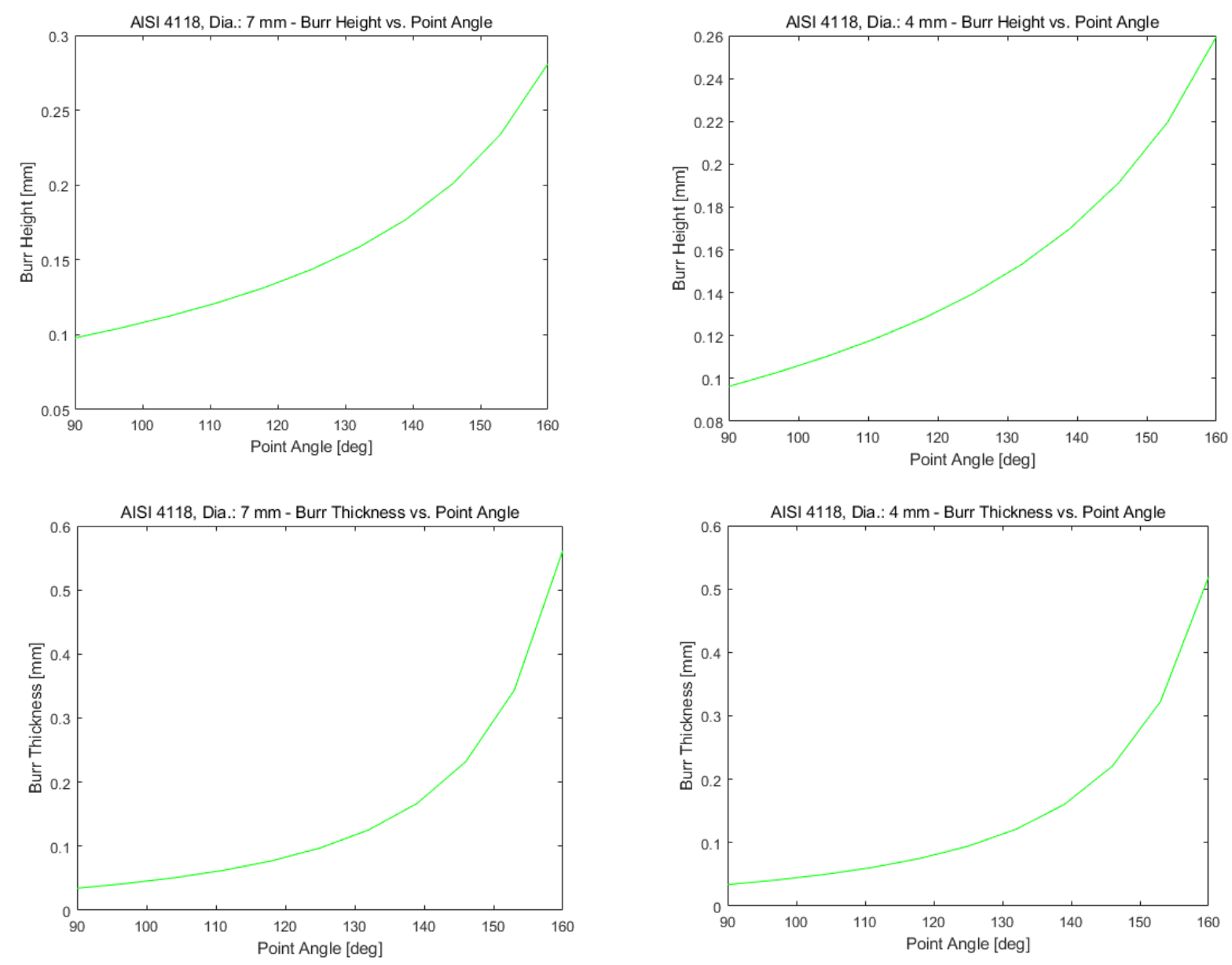

Fig. B.4 Burr heights and thicknesses vs. point angle from AISI 4118 


\section{APPENDIX C: BURR SENSITIVITY MODEL - DIFFERENTIATION OF $\Phi$ WITH RESPECT TO FEED $\partial \Phi / \partial f$ AND WITH RESPECT TO POINT ANGLE $\partial \Phi / \partial p$}

\section{C.1 Differentiation of $\Phi$ with Respect to Feed}

Starting with

$$
\Phi=\sum_{i=1}^{N}\left\{\frac{\sin \left(\frac{\pi}{6}-\frac{\alpha_{d}}{2}\right)\left(\rho_{i+1-} \rho_{i}\right)}{\sin \left(\frac{\pi}{6}+\frac{\alpha_{d}}{4}\right) \cos \left(\frac{\pi}{3}-\frac{\alpha_{d}}{4}\right)}\right\}
$$

Split temporarily into subcomponents as follows

$$
\begin{gathered}
\Phi_{1}=\sin \left(\frac{\pi}{6}-\frac{\alpha_{d}}{2}\right)\left(\rho_{i+1-} \rho_{i}\right) \\
\Phi_{2}=\frac{1}{\sin \left(\frac{\pi}{6}+\frac{\alpha_{d}}{4}\right)} \\
\Phi_{3}=\frac{1}{\cos \left(\frac{\pi}{3}-\frac{\alpha_{d}}{4}\right)}
\end{gathered}
$$

Revisiting $\alpha_{d}$ and $\alpha_{n}$

$$
\begin{gathered}
\alpha_{d}=\alpha_{n}+\tan ^{-1}\left(\frac{f}{2 \pi r_{i}}\right) \\
\alpha_{n}=\tan ^{-1}\left\{\frac{\tan \delta_{0}\left(\rho^{2}-w^{2} \sin ^{2} p\right)-w \sin p \cos p}{\sin p\left(\rho^{2}-w^{2}\right)^{\frac{1}{2}}}\right\}
\end{gathered}
$$

The differentiation of $\Phi$ with respect to feed starts with the differentiation of $\alpha_{d}$ with respect to feed; $\alpha_{n}$ does not have a feed component and is, therefore, unaffected

$$
\frac{\partial \alpha_{d}}{\partial f}=\left(\frac{1}{\left(\left(\frac{f}{2} \pi r_{i}\right)^{2}+1\right)}\right)\left(1 /\left(2 \pi r_{i}\right)\right.
$$


Culminating in:

$$
\begin{aligned}
\frac{\partial \Phi}{\partial f}=\sum\left[\left(-\frac{1}{2}(\right.\right. & \left.\left.\left.\cos \left(\frac{\pi}{6}-\frac{\alpha_{d}}{2}\right)\right) \cdot\left(\rho_{i+1-} \rho_{i}\right) \cdot \frac{\partial \alpha_{d}}{\partial f}\right)\left(\frac{1}{\sin \left(\frac{\pi}{6}+\frac{\alpha_{d}}{4}\right)}\right)\left(\frac{1}{\cos \left(\frac{\pi}{3}-\frac{\alpha_{d}}{4}\right)}\right)\right] \\
+ & {\left[( \operatorname { s i n } ( \frac { \pi } { 6 } - \frac { \alpha _ { d } } { 2 } ) ( \rho _ { i + 1 - } \rho _ { i } ) ) \left(-\frac{1}{4}\left(\cot \left(\frac{\pi}{6}+\frac{\alpha_{d}}{4}\right)\right) \cdot\left(\csc \left(\frac{\pi}{6}+\frac{\alpha_{d}}{4}\right)\right)\right.\right.} \\
& \left.\left.\cdot \frac{\partial \alpha_{d}}{\partial f}\right)\left(\frac{1}{\cos \left(\frac{\pi}{3}-\frac{\alpha_{d}}{4}\right)}\right)\right] \\
+ & {\left[( \operatorname { s i n } ( \frac { \pi } { 6 } - \frac { \alpha _ { d } } { 2 } ) ( \rho _ { i + 1 - } \rho _ { i } ) ) ( \frac { 1 } { \operatorname { s i n } ( \frac { \pi } { 6 } + \frac { \alpha _ { d } } { 4 } ) } ) \left(-\frac{1}{4} \cot \left(\frac{\pi}{6}+\frac{\alpha_{d}}{4}\right)\right.\right.} \\
& \left.\left.\cdot\left(\csc \left(\frac{\pi}{6}+\frac{\alpha_{d}}{4}\right)\right) \cdot \frac{\partial \alpha_{d}}{\partial f}\right)\right]
\end{aligned}
$$

\section{C.2 Differentiation of $\Phi$ with Respect to Point Angle}

Once again starting with

$$
\Phi=\sum_{i=1}^{N}\left\{\frac{\sin \left(\frac{\pi}{6}-\frac{\alpha_{d}}{2}\right)\left(\rho_{i+1-} \rho_{i}\right)}{\sin \left(\frac{\pi}{6}+\frac{\alpha_{d}}{4}\right) \cos \left(\frac{\pi}{3}-\frac{\alpha_{d}}{4}\right)}\right\}
$$

Split temporarily into subcomponents as follows:

$$
\begin{gathered}
\Phi_{1}=\sin \left(\frac{\pi}{6}-\frac{\alpha_{d}}{2}\right)\left(\rho_{i+1-} \rho_{i}\right) \\
\Phi_{2}=\frac{1}{\sin \left(\frac{\pi}{6}+\frac{\alpha_{d}}{4}\right)} \\
\Phi_{3}=\frac{1}{\cos \left(\frac{\pi}{3}-\frac{\alpha_{d}}{4}\right)}
\end{gathered}
$$

Revisiting $\alpha_{d}$ and $\alpha_{n}$

$$
\begin{gathered}
\alpha_{d}=\alpha_{n}+\tan ^{-1}\left(\frac{f}{2 \pi r_{i}}\right) \\
\alpha_{n}=\tan ^{-1}\left\{\frac{\tan \delta_{0}\left(\rho^{2}-w^{2} \sin ^{2} p\right)-w \sin p \cos p}{\sin p\left(\rho^{2}-w^{2}\right)^{\frac{1}{2}}}\right\}
\end{gathered}
$$


The differentiation of $\alpha_{d}$ with respect to the point angle $p$ incorporates $\frac{\partial \alpha_{n}}{\partial p}$ :

$$
\begin{aligned}
& \frac{\partial \alpha_{d}}{\partial p}= \\
& \frac{\left(\left(-(\cot p \csc p) *\left(\left(\rho^{2}\right)-(w \cos p \sin p)-\left(w^{2} \sin ^{2} p\right)\right)+\csc p *\left(-\left(2 w^{2} \cos p \sin p\right)-\left(w\left(\cos ^{2} p-\sin ^{2} p\right)\right)\right) * \tan \delta\right)\right.}{\left(\left(\sqrt{\rho^{2}-w^{2}}\right)\left(1+\frac{\left(\csc ^{2} p *\left(\rho-(w \cos p \sin p)-\left(w^{2} \sin ^{2} p\right)\right)^{2} \tan \delta\right)}{\left(\rho^{2}-w^{2}\right)}\right)\right)} \\
& \begin{array}{c}
\frac{\partial \Phi}{\partial p}=\sum\left(\rho_{i+1}-\rho_{i}\right)\left(-\frac{1}{2} \frac{\partial \alpha_{d}}{\partial p}\left(\cot \left(\frac{\pi}{6}+\frac{\alpha_{d}}{4}\right)\right)\left(\csc ^{2}\left(\frac{\pi}{6}+\frac{\alpha_{d}}{4}\right)\right)\left(\sin \left(\frac{\pi}{6}-\frac{\alpha_{d}}{2}\right)\right)\right. \\
\left.-\frac{1}{2}\left(\cos \left(\frac{\pi}{6}-\frac{\alpha_{d}}{2}\right)\right)\left(\csc ^{2}\left(\frac{\pi}{6}+\frac{\alpha_{d}}{4}\right)\right) \frac{\partial \alpha_{d}}{\partial p}\right)
\end{array}
\end{aligned}
$$




\section{APPENDIX D: BURR SENSITIVITY MODEL PLOTS}

D.1 2D Evaluation of Sensitivity Analysis Plots
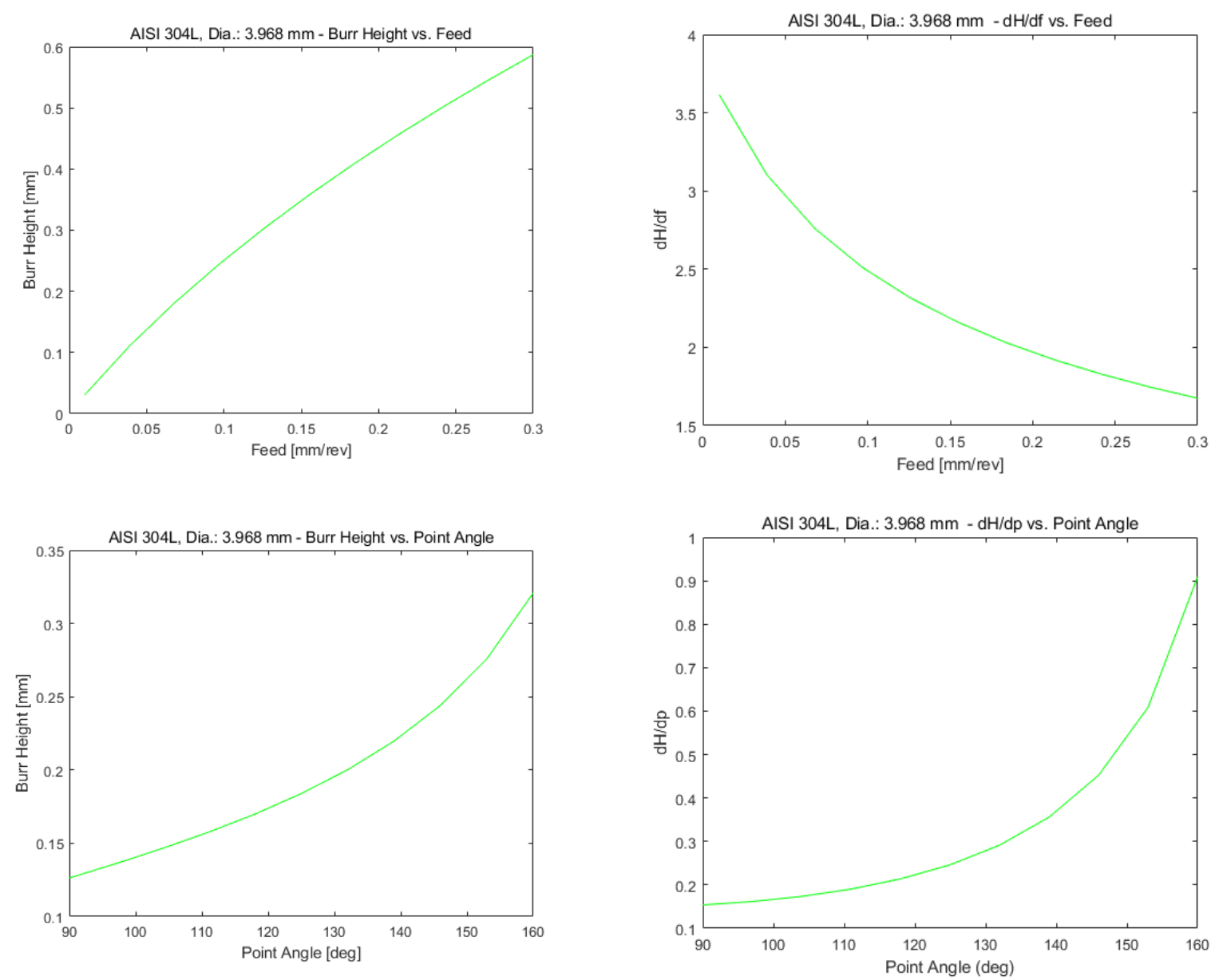

Fig. D.1 Burr height and rate of change of burr height for AISI 304L, $3.968 \mathrm{~mm}$ dia. drill bit 

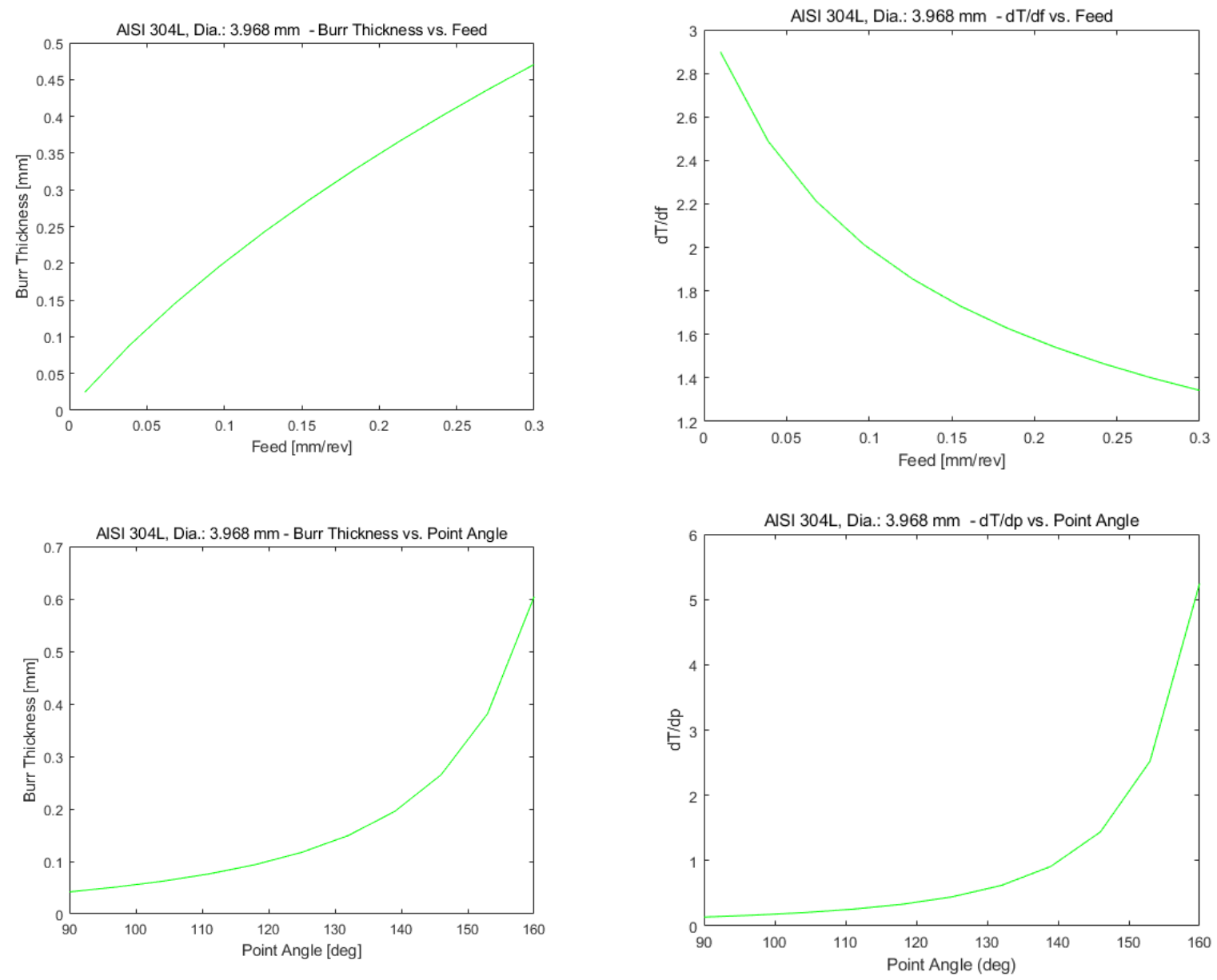

Fig. D.2 Burr thickness and rate of change of burr thickness for AISI 304L, 3.968mm dia. drill bit 

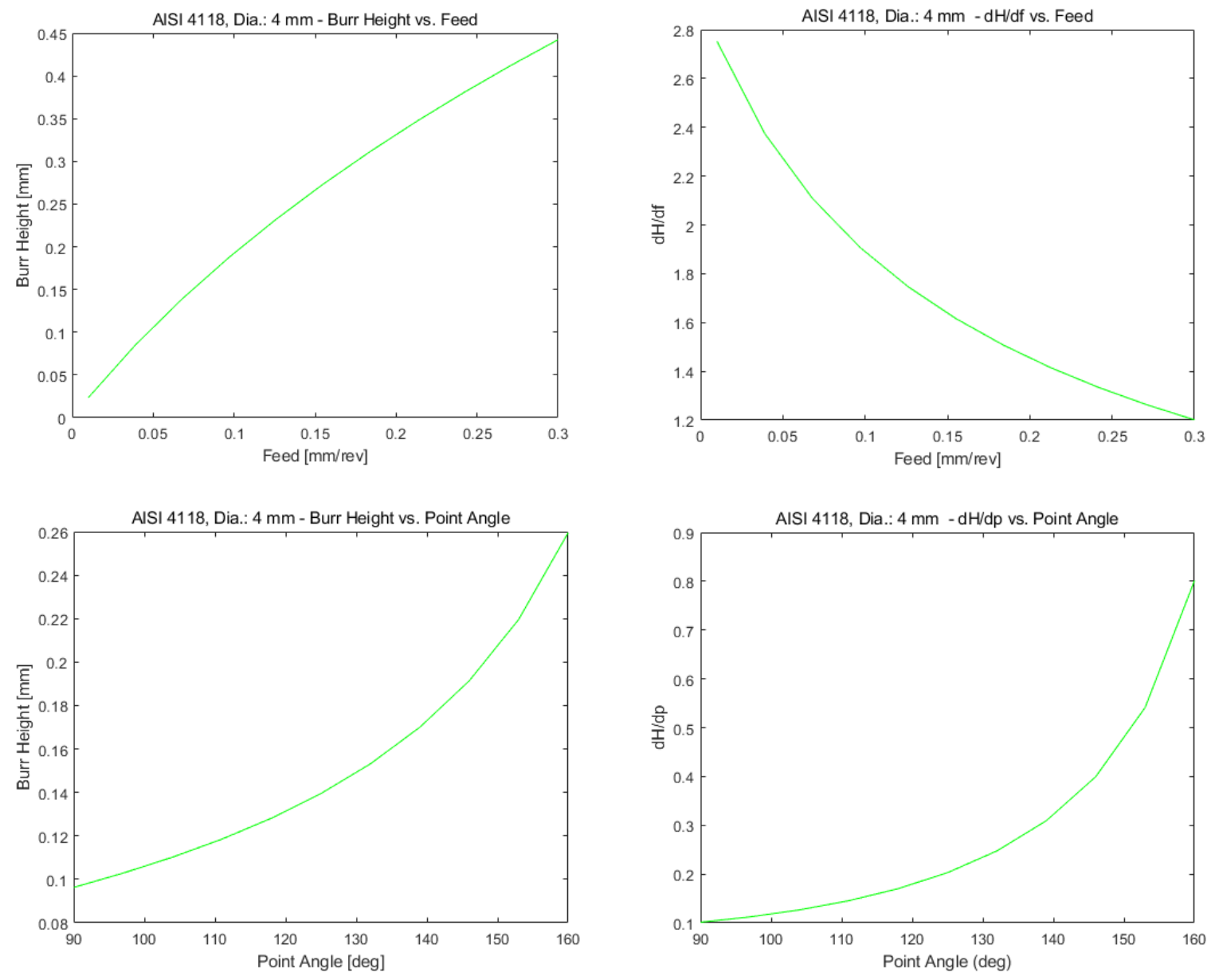

Fig. D.3 Burr height and rate of change of burr height for AISI 4118, $4 \mathrm{~mm}$ dia. drill bit 

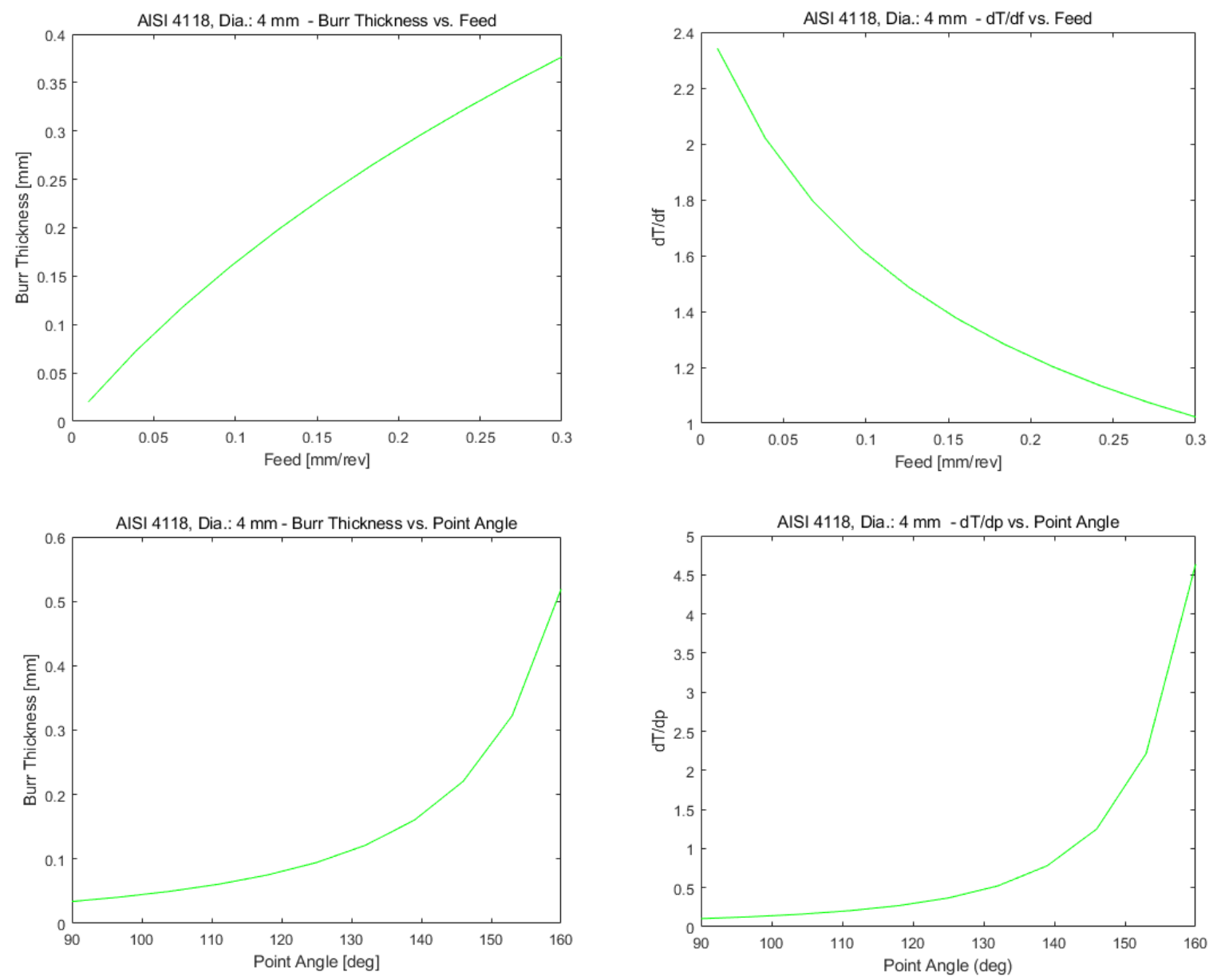

Fig. D.4 Burr thickness and rate of change of burr thickness for AISI 4118, $4 \mathrm{~mm}$ dia. drill bit 

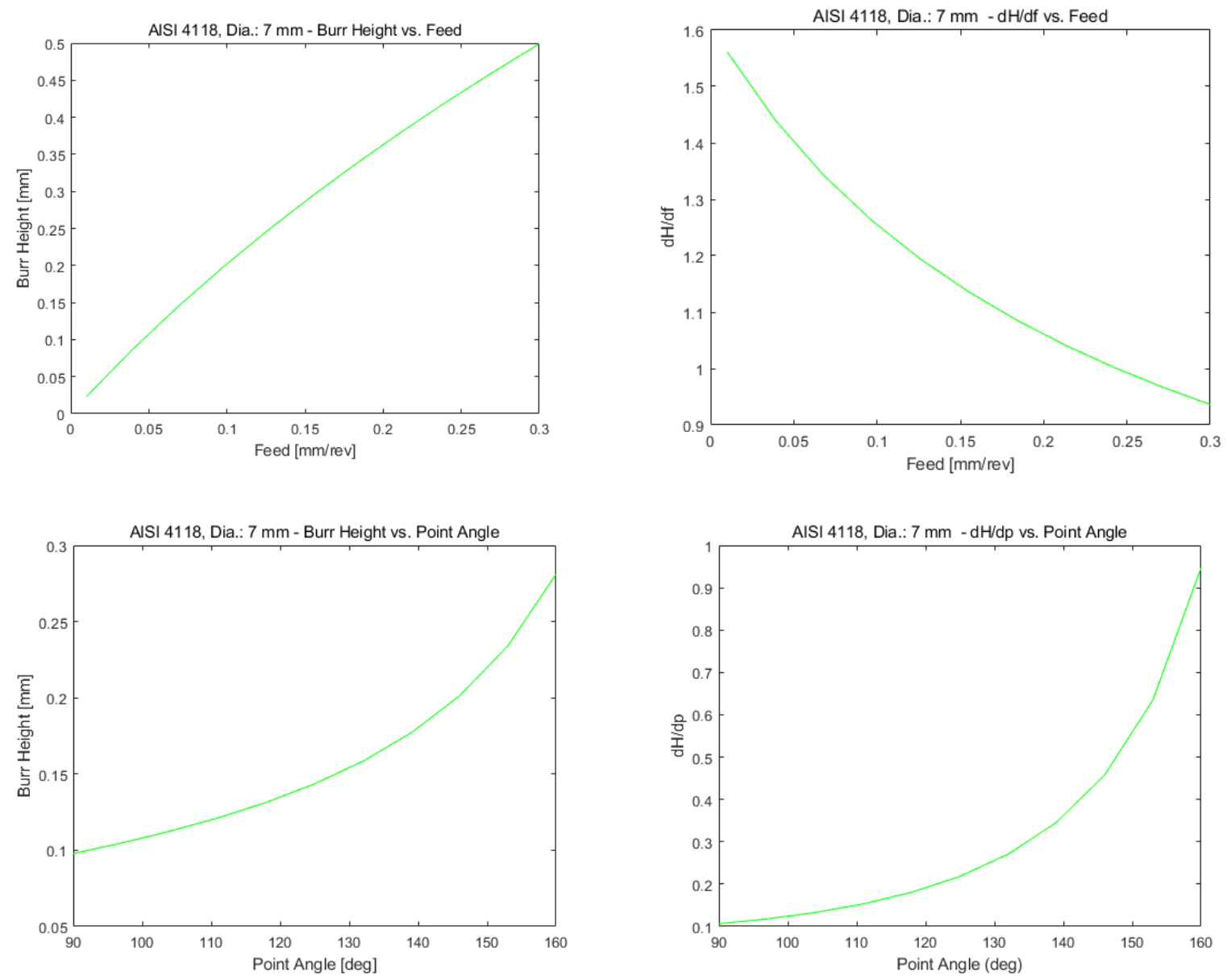

Fig. D.5 Burr height and rate of change of burr height for AISI 4118, $7 \mathrm{~mm}$ dia. drill bit 

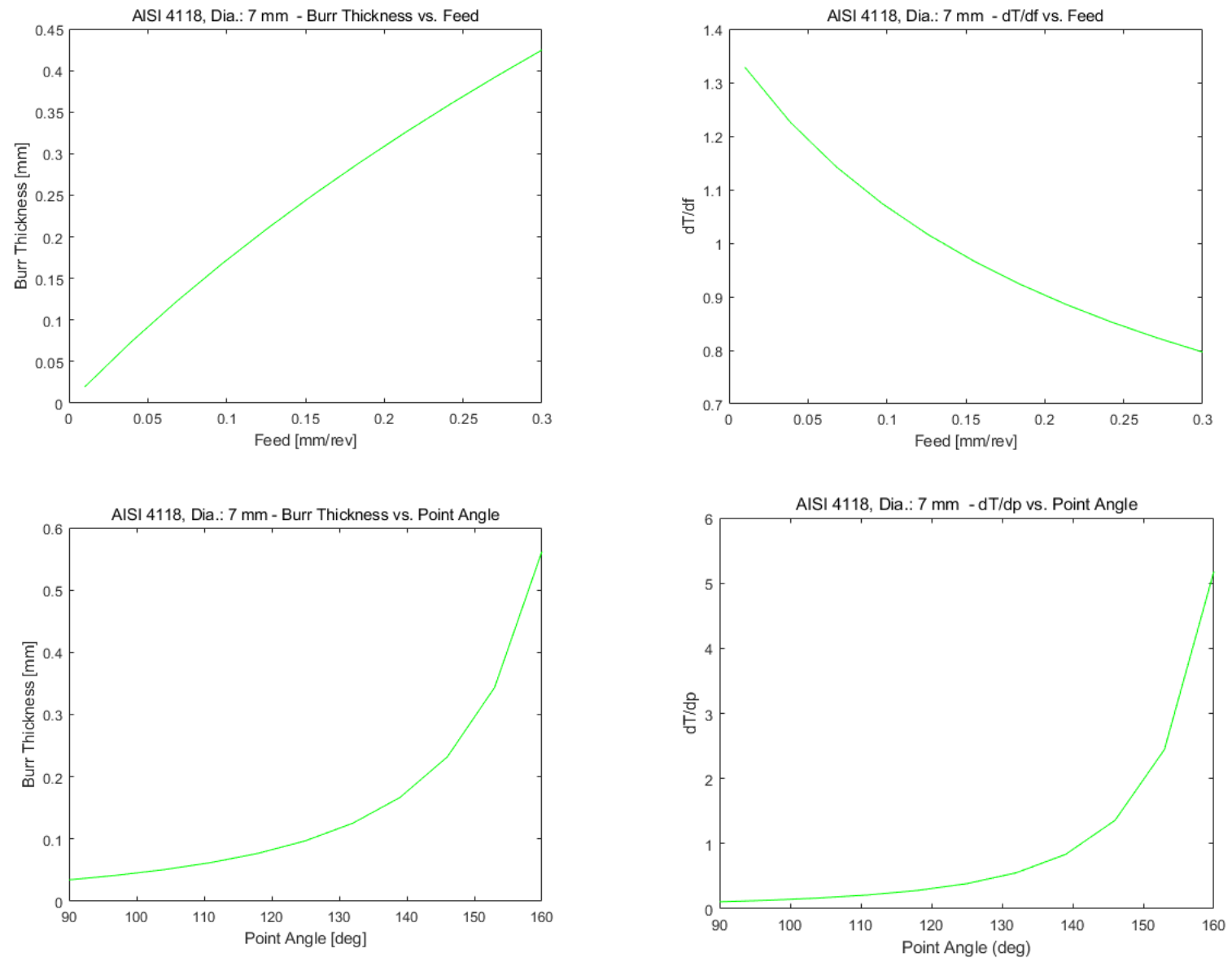

Fig. D.6 Burr thickness and rate of change of burr thickness for AISI 4118, $7 \mathrm{~mm}$ dia. drill bit 

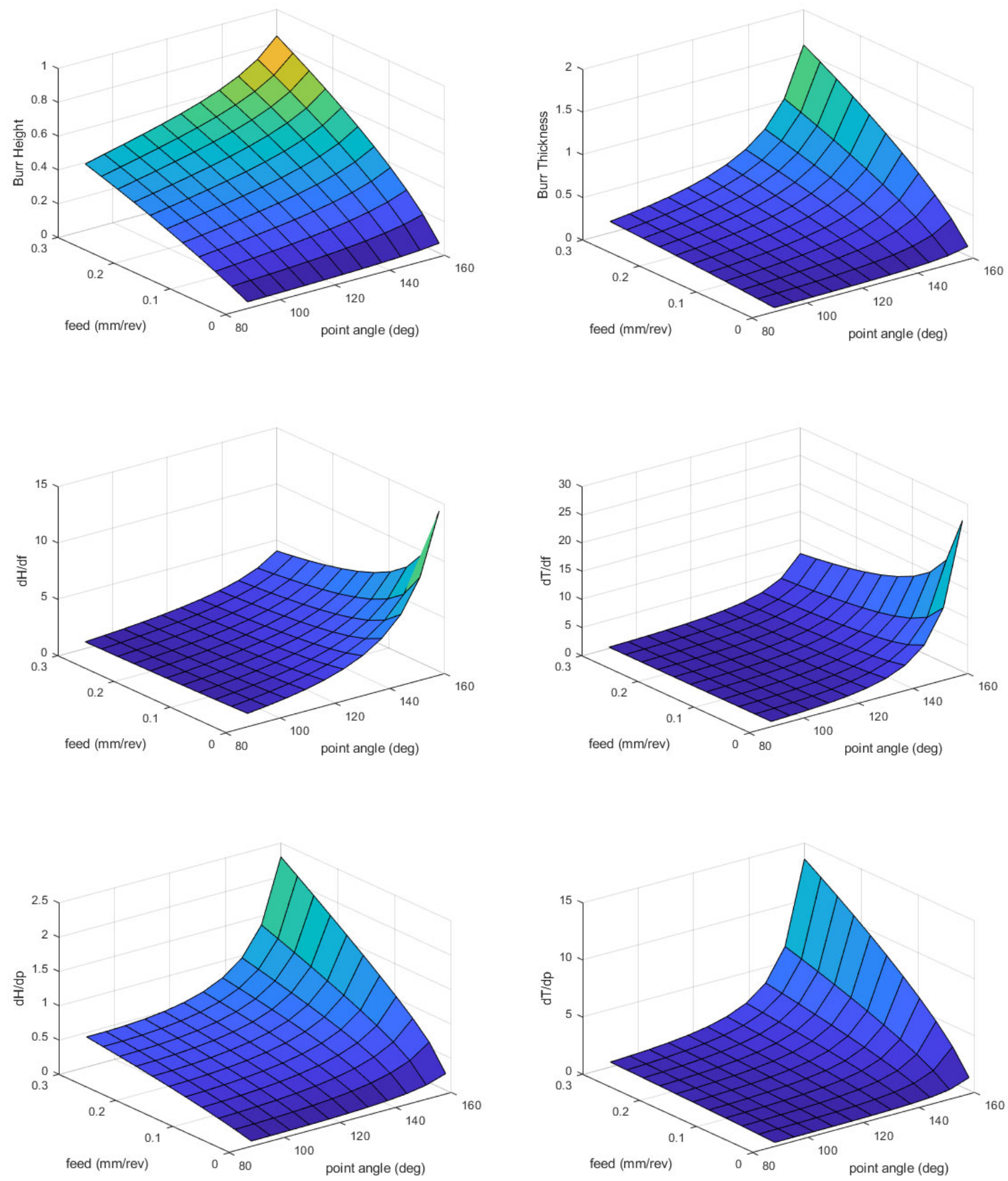

Fig. D.7 AISI 304L dia. 3.968mm - surface plots of burr height, thickness, and respective rates of change of height and thickness vs. point angle and feed 

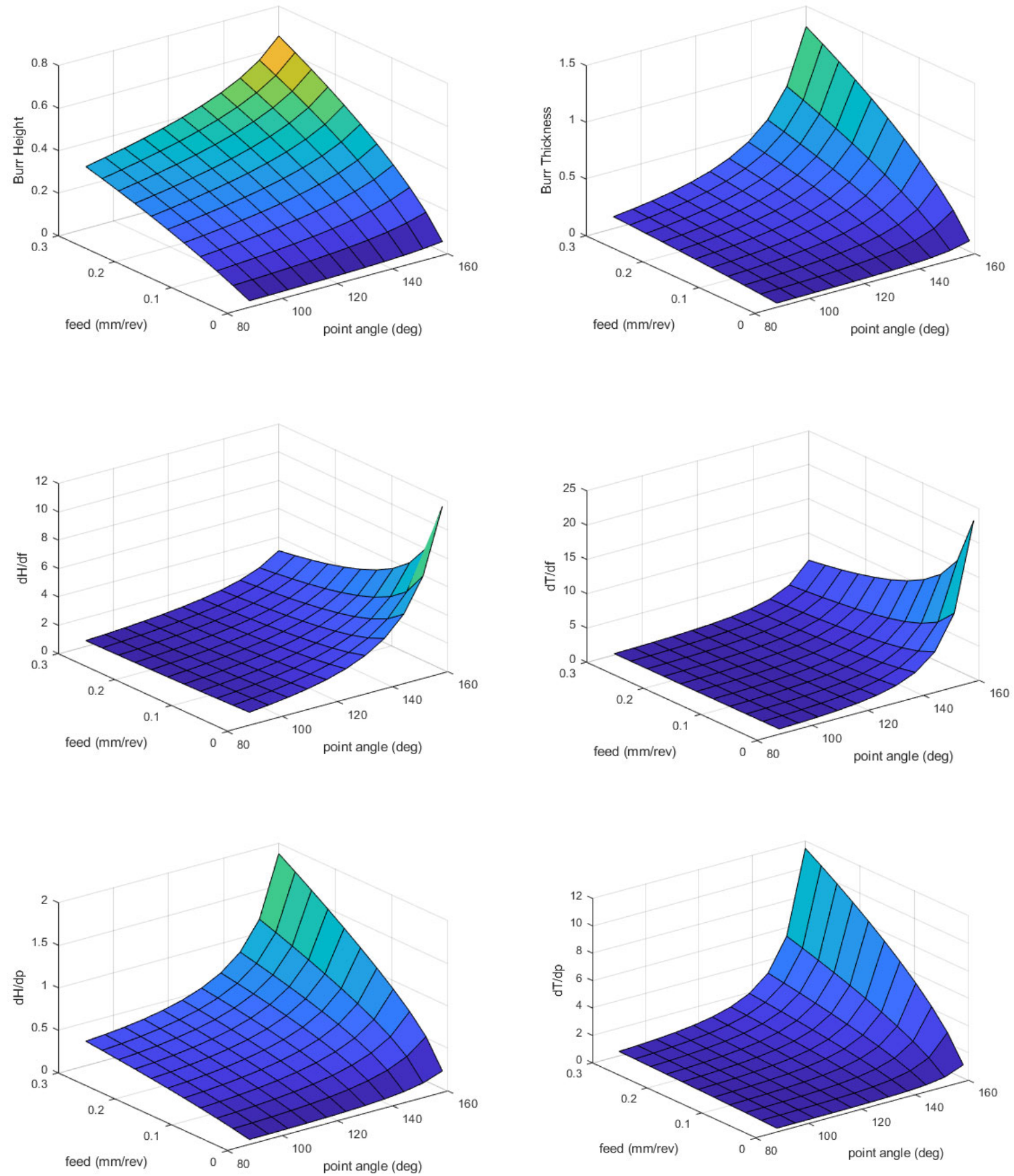

Fig. D.8 AISI 4118 dia. $4 \mathrm{~mm}$ - surface plots of burr height, thickness, and respective rates of change of height and thickness vs. point angle and feed 

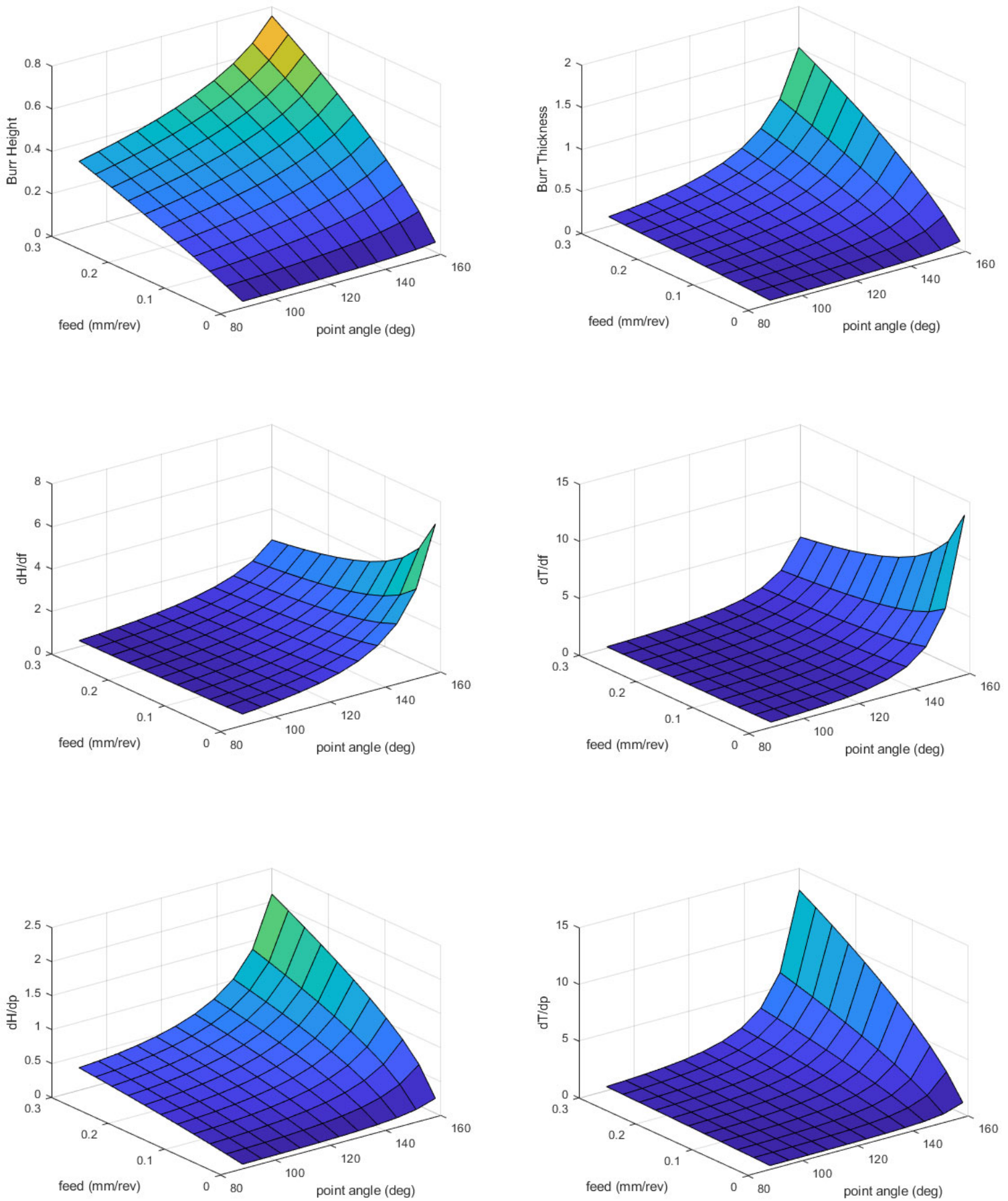

Fig. D.9 AISI 4118 dia. $7 \mathrm{~mm}$ - surface plots of burr height, thickness, and respective rates of change of height and thickness vs. point angle and feed 
Table D.1 Objective function results, continued

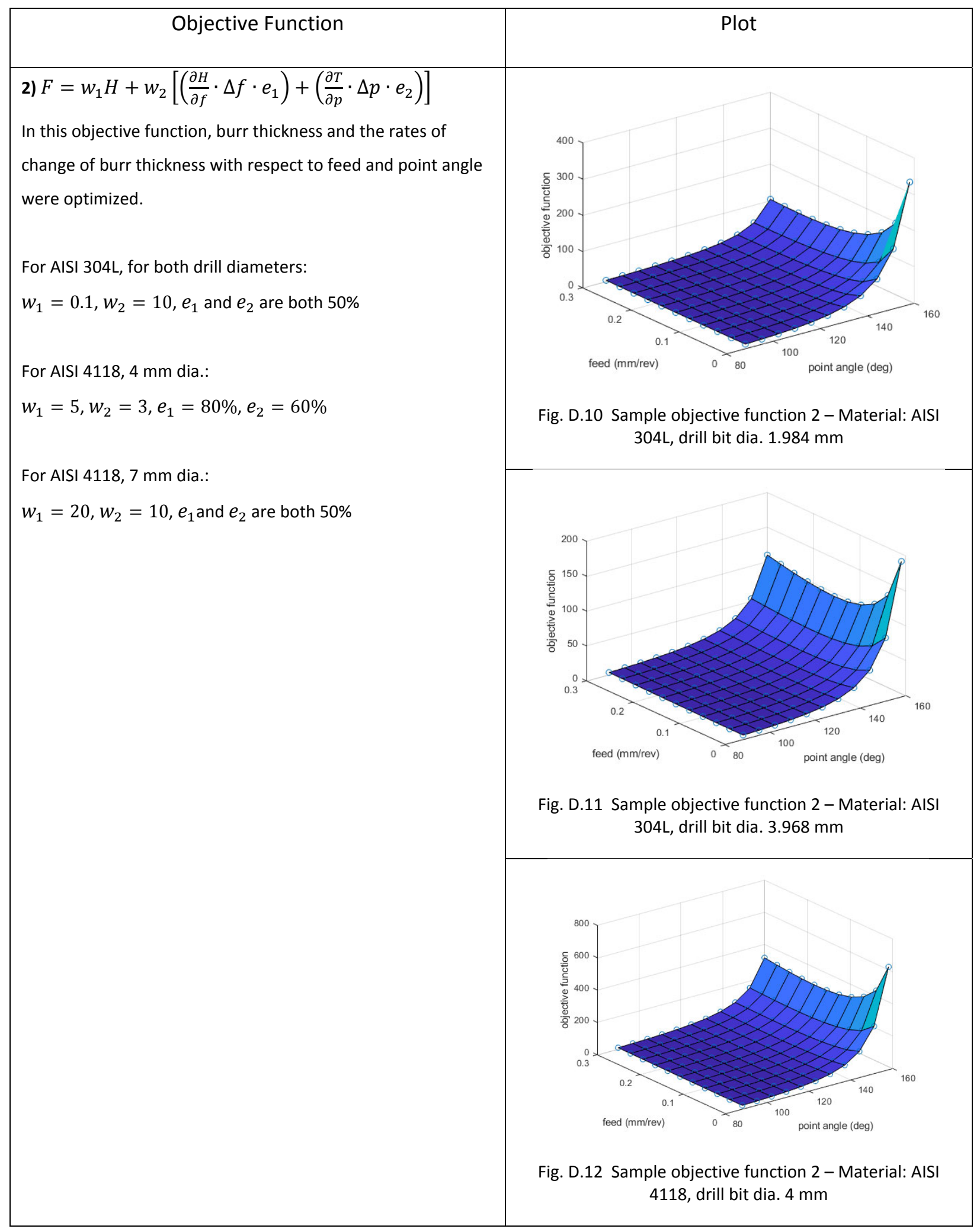




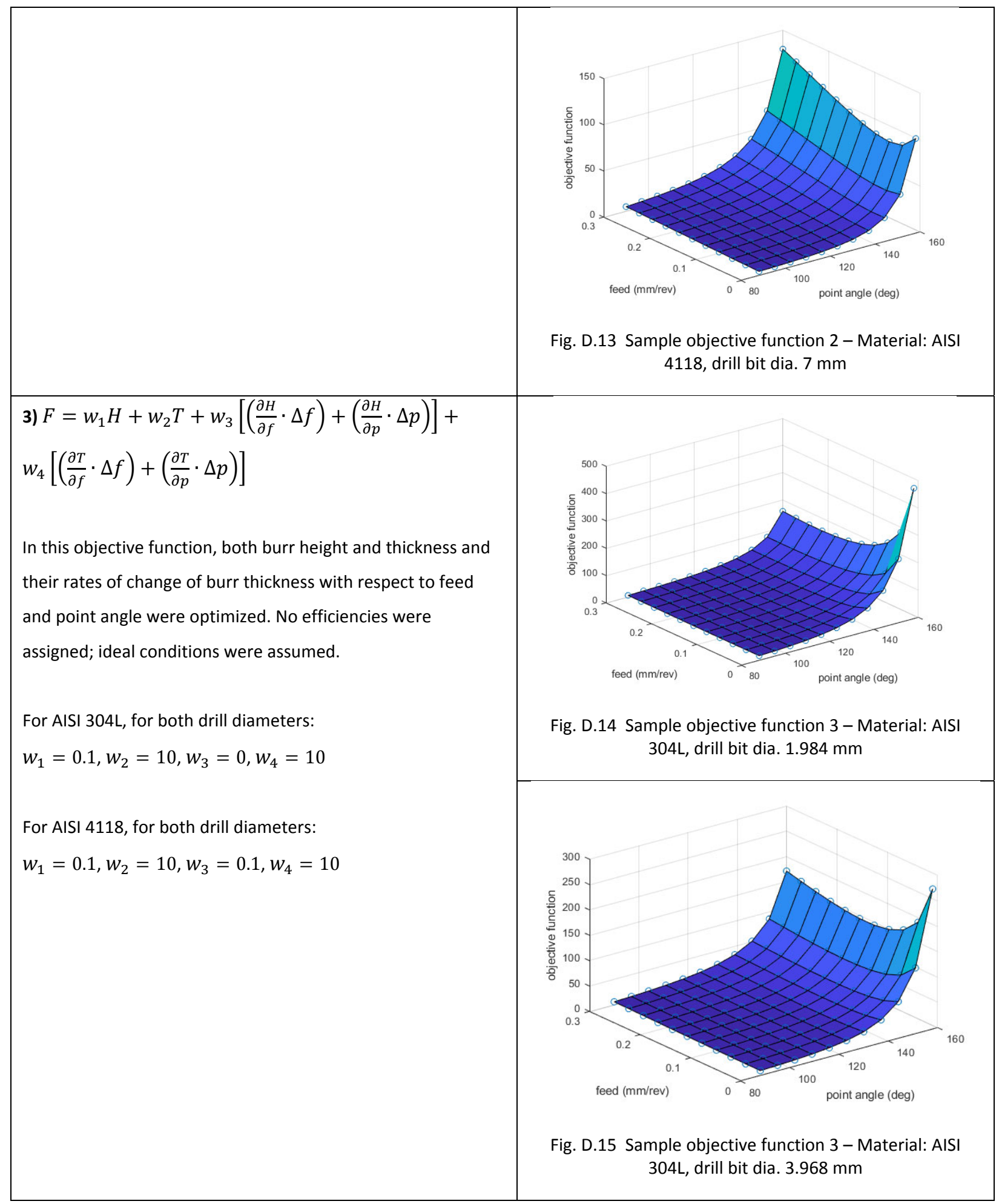




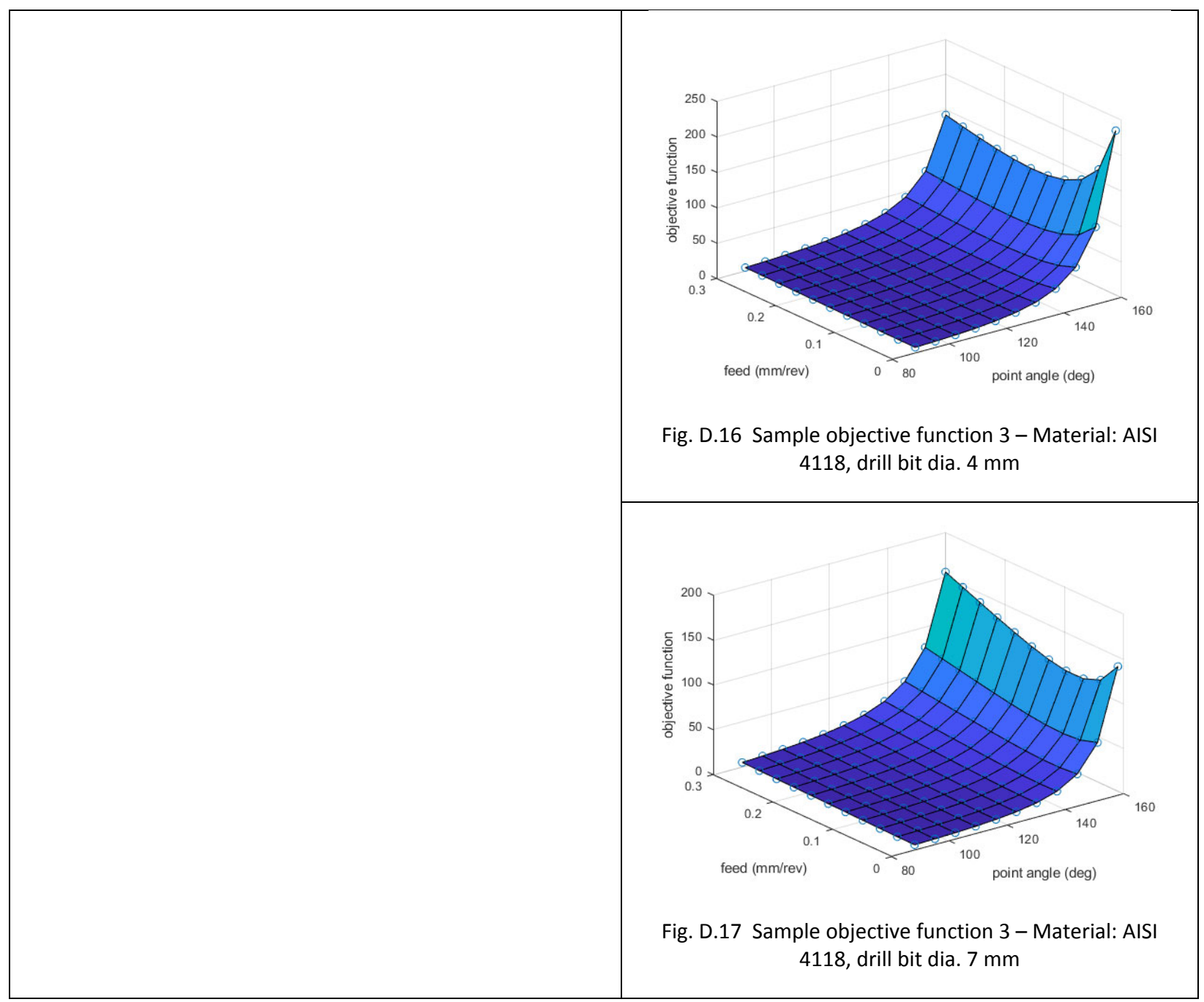




\section{APPENDIX E: PLOUGHING THURST FORCE - DETAILED DERIVATION}

\section{E.1. Ploughing Thrust Force}

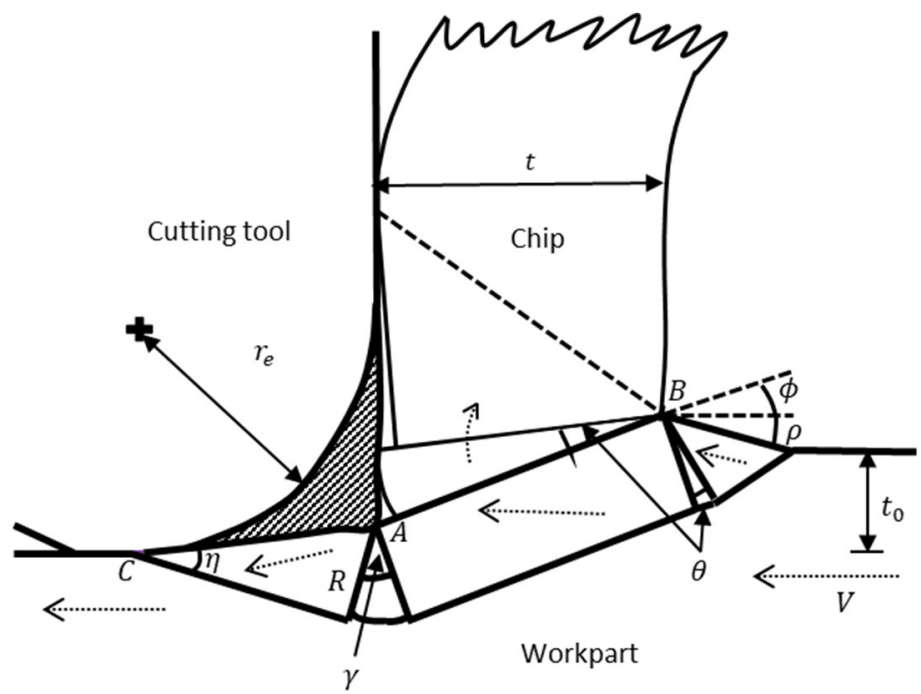

Fig. 4.1 Slip-line field model for ploughing in orthogonal cutting, adapted from [15, Fig. 3]

The ploughing thrust force, eq. (4.1), developed by [15] is as follows:

$$
F_{p l}=k w[(1+2 \sin \theta+2 \gamma+\sin (2 \eta)) \cos (\phi-\gamma+\eta)-\cos (2 \eta) \sin (\phi-\gamma+\eta)] C A
$$

The two key force parameters are $k$, the material shear strength, and $w$, the contact region width defined below. The angles are actually angle projections, called fan field angles $\theta$ and $\gamma$, eq. (E.1), [15, eq. (4)] and eq. (E.2), [15, eq. (5)]:

$$
\begin{gathered}
\theta=\frac{\pi}{4}-\rho_{w}-\phi \\
\gamma=\eta+\phi-\sin ^{-1}\left(\sqrt{2} \cdot \sin \left(\rho_{w}\right) \cdot \sin (\eta)\right)
\end{gathered}
$$

$\eta$ is the angle between the slip line and the bottom surface of the built-up region, as per eq. (E.3) [15, eq. (3)]:

$$
\eta=0.5 \cdot \cos (m)^{-1}
$$


$m$ is the friction factor, from the constant frictional stress relation, as per eq. (E.4) [15, eq. (1)]:

$$
\tau=m * k
$$

The interdependence in eq. (E.4) stems from a high normal stress in the hatched region between the tool and the work in Fig. 4.1 ( $C A$ region). In the ploughing force model, the authors assume $\tau$ to be proportional to the material shear flow stress, identified as $k$. In eq. (E.5) [15, eq. (2)], shear flow stress of work material, $k$, is part of the primary deformation zone in orthogonal cutting. It can be applied in slip-line field and shear plane examinations for continuous chip formation [15].

$$
k=\frac{Y}{\sqrt{3}}
$$

In eq. (E.5), $Y$ is the uniaxial yield stress. For the purposes of this dissertation, where drilling and burr formation are at issue, $k$ has been redefined to represent material shear strength at the shear plane, instead of shear flow. In accordance with the Von Mises stress criterion, $\sigma_{y}$ replaces $Y$ in the shear strength relation as follows:

$$
k=\frac{\sigma_{y}}{\sqrt{3}}
$$

As per Figure 4.1, $C A$, eq. (E.7) [15, eq. (17)] represents both the workpiece and the bottom surface of the material buildup region, as well as the zone where ploughing forces act.

$$
C A=\frac{R_{\operatorname{circ}}}{\sin (\eta)}
$$

A component important to the new model developed in Chapter 4 is $R_{c i r c}$, a subcomponent of $C A$, eq. (E.8) [15, eq. (6)]. $R_{\text {circ }}$ is the radius of the circular fan field with its centre at $A$ :

$$
\begin{aligned}
& R_{\text {circ }} \\
& =\sin (\eta) \times \sqrt{\left.\left(r_{e} \cdot \tan \left(\frac{\pi}{4}+\frac{\alpha}{2}\right)+\frac{\sqrt{2} \cdot R \cdot \sin \left(\rho_{\text {row }}\right)}{\tan \left(\frac{\pi}{2}+\alpha\right)}\right)^{2}+2 \cdot\left[R_{\text {circ }} \cdot \sin \left(\rho_{\text {row }}\right)\right)\right]^{2}}
\end{aligned}
$$


where $r_{e}$ is the tool cutting edge radius, which will be a critical parameter to the new tool wear model.

For the purposes of the development of the ploughing force model, the prow angle is set to approach zero, which leads $R_{\text {circ }}$ to simplify to:

$$
R_{\text {circ }}=\sin (\eta) \times \sqrt{\left(r_{e} \cdot \tan \left(\frac{\pi}{4}+\frac{\alpha}{2}\right)\right)^{2}}
$$




\section{APPENDIX F: PLOUGHING FORCE AND NEW COMBINED DRILLING THRUST FORCE MODEL FLOWCHART}

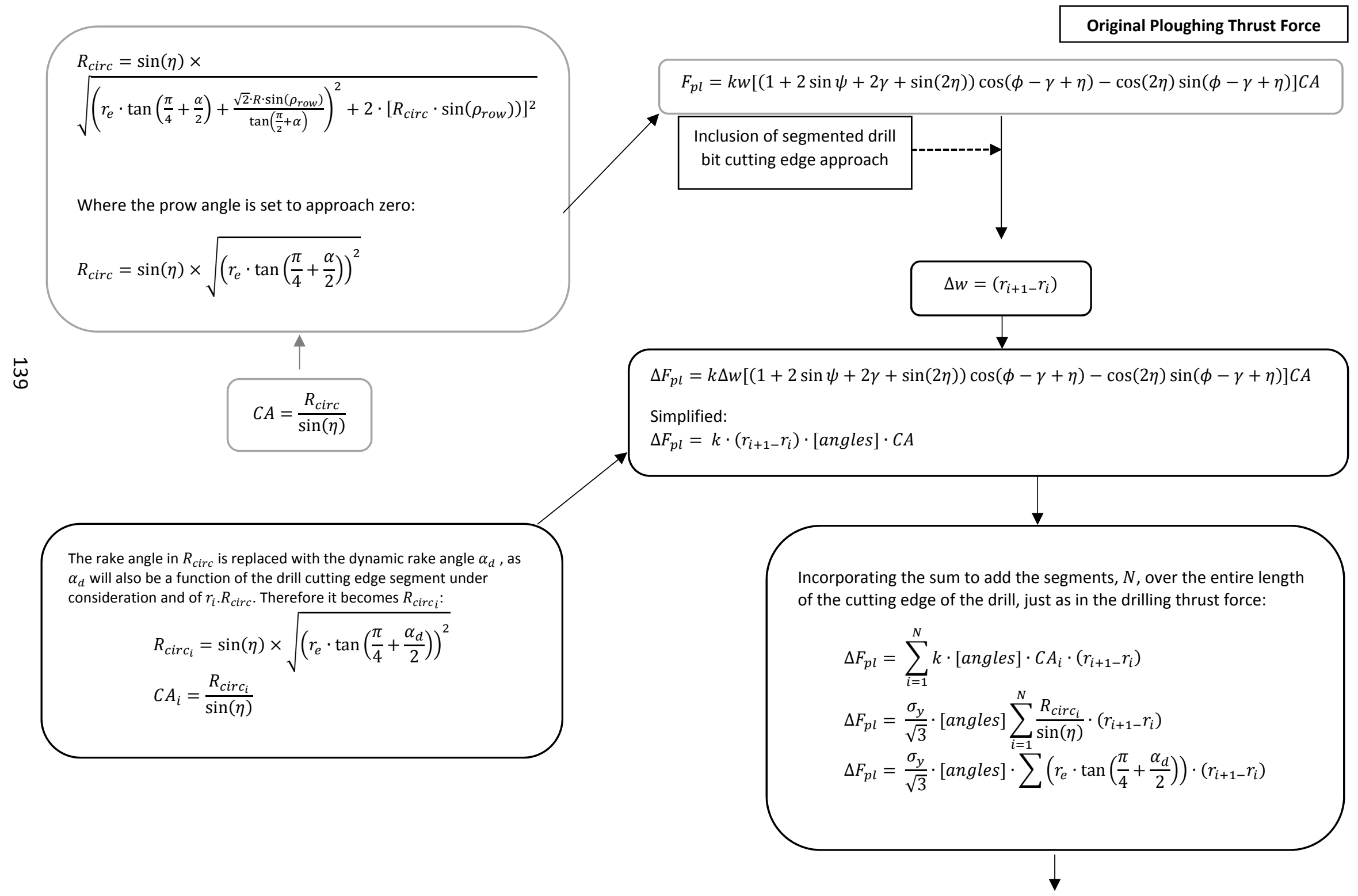




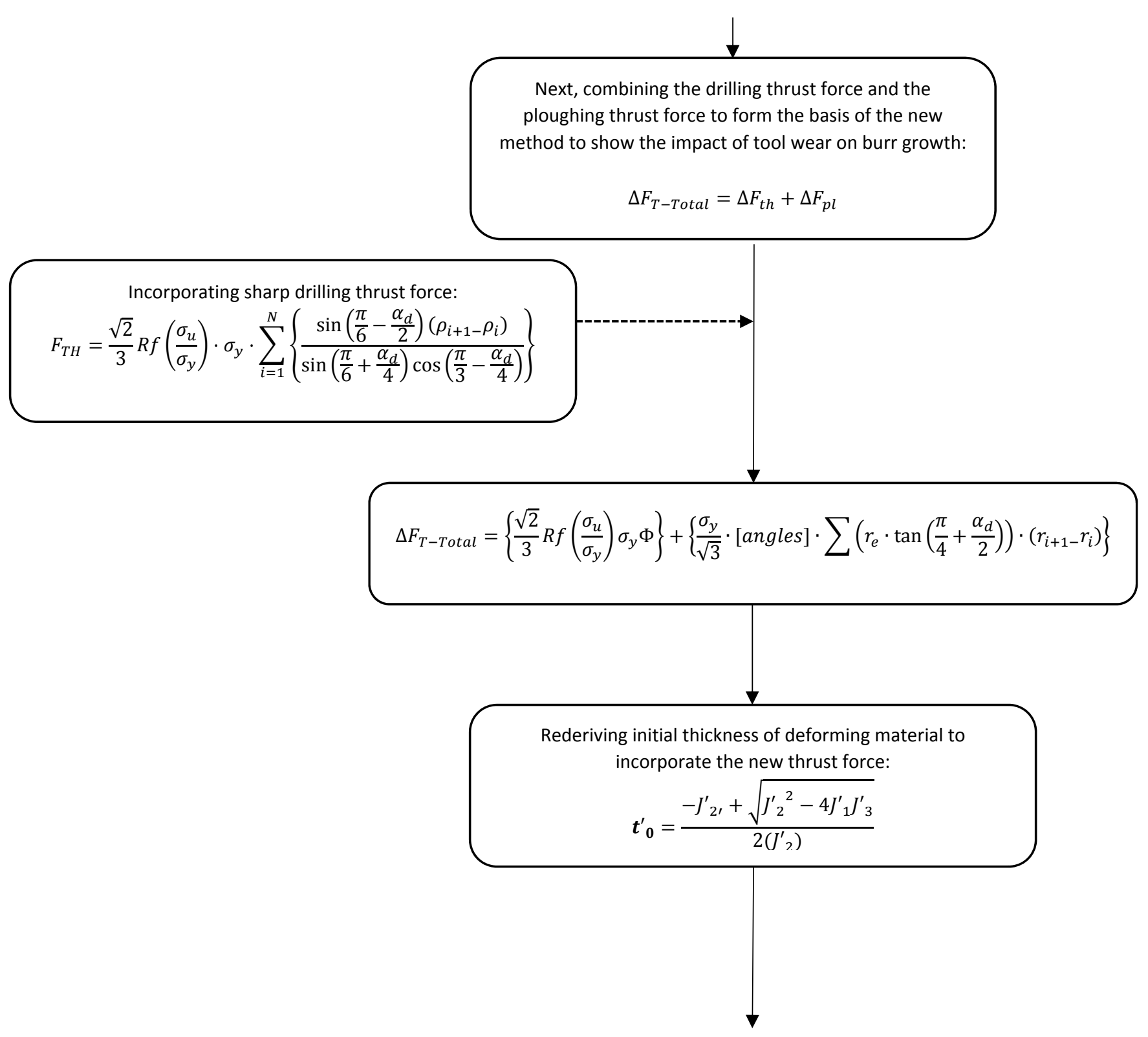




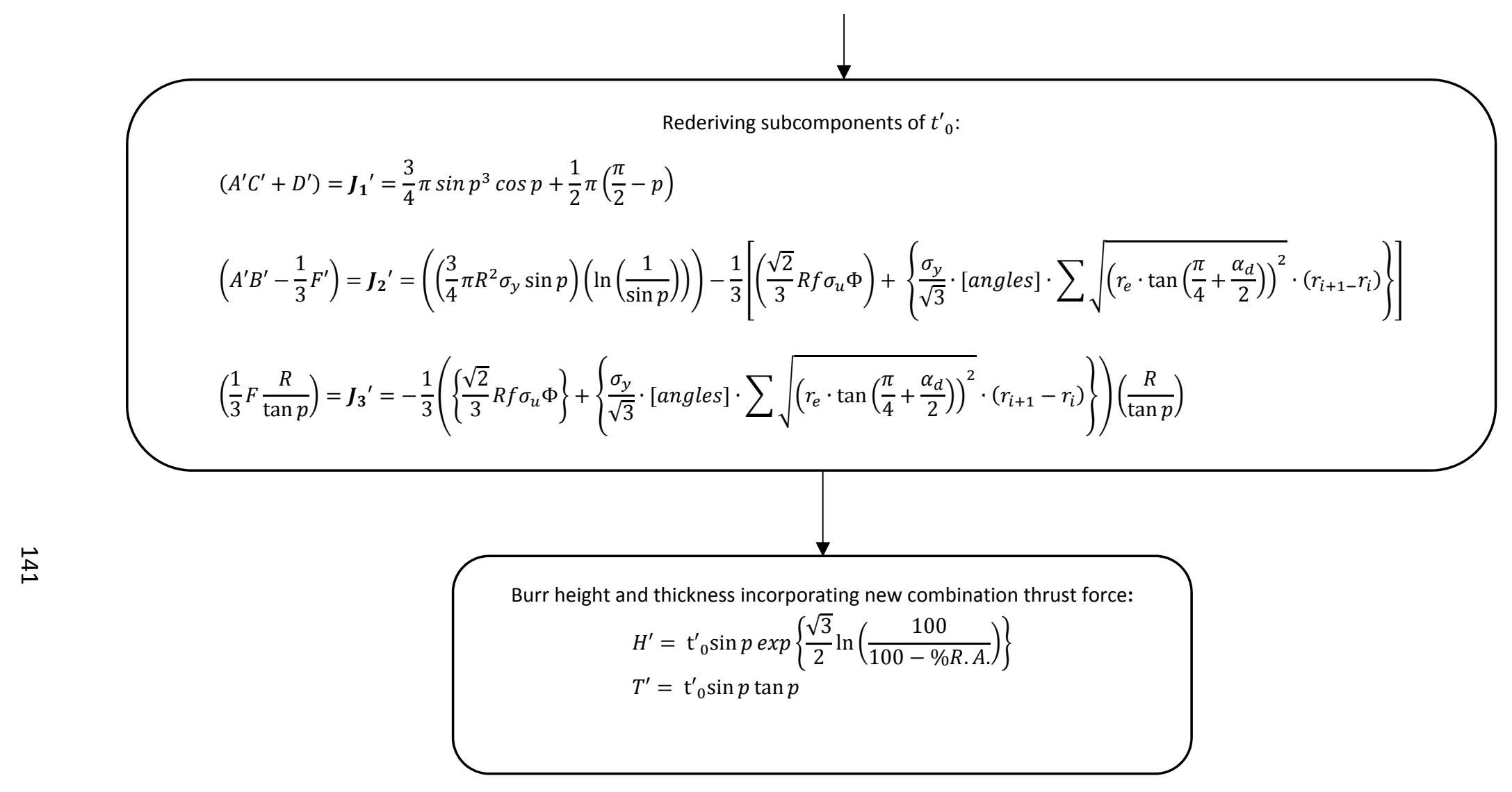

Fig. F.1 Ploughing force and new combined drilling thrust force model flowchart 
APPENDIX G: EXPERIMENTAL DATA FROM DRILLING AL 6061 T6 AND AL 7075 T6

G.1 Al 6061 T6 Data

Table G.1 Al 6061 T6 burr height data at feed $.002 \mathrm{in} / \mathrm{rev}$

\begin{tabular}{|c|c|c|c|c|c|c|c|c|c|c|}
\hline $\begin{array}{l}\text { Material } \\
\text { thickness } \\
\text { [in] }\end{array}$ & \begin{tabular}{|l|} 
Level of \\
drill bit \\
wear
\end{tabular} & $\begin{array}{l}\text { Height } 1 \\
{[\mu \mathrm{m}]}\end{array}$ & $\begin{array}{l}\text { Height } 2 \\
{[\mu \mathrm{m}]}\end{array}$ & $\begin{array}{l}\text { Height } 3 \\
{[\mu \mathrm{m}]}\end{array}$ & $\begin{array}{l}\text { Height } 4 \\
{[\mu \mathrm{m}]}\end{array}$ & $\begin{array}{l}\text { Height } 5 \\
{[\mu \mathrm{m}]}\end{array}$ & $\begin{array}{l}\text { Mean burr } \\
\text { height } \\
{[\mu \mathrm{m}]}\end{array}$ & $\begin{array}{l}\text { Standard } \\
\text { deviation }\end{array}$ & $\begin{array}{l}\text { Simulation } \\
\text { burr height } \\
{[\mu \mathrm{m}]}\end{array}$ & $\begin{array}{l}\text { Delta between } \\
\text { simulation and } \\
\text { experimental } \\
\text { burr height } \\
{[\mu \mathrm{m}]}\end{array}$ \\
\hline \multirow{6}{*}{$\stackrel{\vec{A}}{N}$} & $0 \%$ & 300.00 & 207.00 & 247.00 & 167.00 & 233.00 & 230.80 & 44.03 & 30.6 & 200.20 \\
\hline & $25 \%$ & 443.34 & 475.47 & 553.00 & 86.70 & 45.33 & 320.77 & 211.44 & 38.5 & 282.27 \\
\hline & $50 \%$ & 433.00 & 395.33 & 460.00 & 620.00 & 460.00 & 473.67 & 76.91 & 75.7 & 397.97 \\
\hline & $75 \%$ & 253.00 & 255.00 & 227.00 & 213.00 & 247.76 & 239.15 & 16.42 & 153.2 & 85.95 \\
\hline & $75 \%$ & 440.00 & 620.00 & 427.00 & 587.00 & 500.00 & 514.80 & 77.20 & 153.2 & 361.60 \\
\hline & $100 \%$ & 587.00 & 500.00 & 415.47 & 507.00 & 755.92 & 553.08 & 115.04 & 249.7 & 303.38 \\
\hline .090 & $100 \%$ & 413.00 & 489.00 & 467.00 & 993.00 & 380.00 & 548.40 & 225.62 & 249.7 & 298.70 \\
\hline
\end{tabular}


Table G.2 Al 6061 T6 burr height data at feed $.004 \mathrm{in} / \mathrm{rev}$

\begin{tabular}{|c|c|c|c|c|c|c|c|c|c|c|}
\hline $\begin{array}{l}\text { Material } \\
\text { thickness } \\
\text { [in] }\end{array}$ & $\begin{array}{l}\text { Level of } \\
\text { drill bit } \\
\text { wear }\end{array}$ & $\begin{array}{l}\text { Height } 1 \\
{[\mu \mathrm{m}]}\end{array}$ & $\begin{array}{l}\text { Height } 2 \\
{[\mu \mathrm{m}]}\end{array}$ & $\begin{array}{l}\text { Height } 3 \\
{[\mu \mathrm{m}]}\end{array}$ & $\begin{array}{l}\text { Height } 4 \\
{[\mu \mathrm{m}]}\end{array}$ & $\begin{array}{l}\text { Height } 5 \\
{[\mu \mathrm{m}]}\end{array}$ & $\begin{array}{l}\text { Mean burr } \\
\text { height } \\
\text { [ } \mathrm{mm}]\end{array}$ & $\begin{array}{l}\text { Standard } \\
\text { deviation }\end{array}$ & $\begin{array}{l}\text { Simulation } \\
\text { burr height } \\
{[\mu \mathrm{m}]}\end{array}$ & $\begin{array}{l}\text { Delta between } \\
\text { simulation and } \\
\text { experimental } \\
\text { burr height } \\
{[\mu \mathrm{m}]}\end{array}$ \\
\hline .090 & $0 \%$ & 95.40 & 127.00 & 180.00 & 173.00 & 160.00 & 147.08 & 8.29 & 59.3 & 87.78 \\
\hline .040 & $25 \%$ & 247.00 & 240.00 & 213.00 & 107.00 & 113.00 & 184.00 & 61.51 & 61.4 & 122.60 \\
\hline .090 & $50 \%$ & 775.31 & 346.96 & 535.00 & 846.52 & 524.73 & 605.70 & 181.81 & 91.6 & 514.10 \\
\hline .040 & $75 \%$ & 893.00 & 1010.00 & 1080.00 & 864.00 & 1010.00 & 971.40 & 80.57 & 161.7 & 809.70 \\
\hline .090 & $75 \%$ & 845.99 & 788.16 & 1400.29 & 1417.21 & 1300.00 & 1150.33 & 275.64 & 161.7 & 988.63 \\
\hline .040 & $100 \%$ & 659.95 & 1048.18 & 925.23 & 1028.04 & 1165.67 & 965.41 & 170.76 & 252 & 713.41 \\
\hline .090 & $100 \%$ & 1394.73 & 1471.08 & 1336.54 & 1289.38 & 1337.31 & 1365.81 & 62.34 & 252 & 1113.81 \\
\hline
\end{tabular}


Table G.3 Al 6061 T6 burr height data at feed $.006 \mathrm{in} / \mathrm{rev}$

\begin{tabular}{|c|c|c|c|c|c|c|c|c|c|c|}
\hline $\begin{array}{l}\text { Material } \\
\text { thickness } \\
\text { [in] }\end{array}$ & $\begin{array}{l}\text { Level of } \\
\text { drill bit } \\
\text { wear }\end{array}$ & $\begin{array}{l}\text { Height } 1 \\
{[\mu \mathrm{m}]}\end{array}$ & $\begin{array}{l}\text { Height } 2 \\
{[\mu \mathrm{m}]}\end{array}$ & $\begin{array}{l}\text { Height } 3 \\
{[\mu \mathrm{m}]}\end{array}$ & $\begin{array}{l}\text { Height } 4 \\
{[\mu \mathrm{m}]}\end{array}$ & $\begin{array}{l}\text { Height } 5 \\
{[\mu \mathrm{m}]}\end{array}$ & $\begin{array}{l}\text { Mean burr } \\
\text { height } \\
{[\mu \mathrm{m}]}\end{array}$ & $\begin{array}{l}\text { Standard } \\
\text { deviation }\end{array}$ & $\begin{array}{l}\text { Simulation } \\
\text { burr height } \\
{[\mu \mathrm{m}]}\end{array}$ & $\begin{array}{l}\text { Delta between } \\
\text { simulation and } \\
\text { experimental } \\
\text { burr height } \\
{[\mu \mathrm{m}]}\end{array}$ \\
\hline .090 & $0 \%$ & 95.40 & 127.00 & 180.00 & 173.00 & 160.00 & 147.08 & 8.29 & 59.3 & 87.78 \\
\hline .040 & $25 \%$ & 247.00 & 240.00 & 213.00 & 107.00 & 113.00 & 184.00 & 61.51 & 61.4 & 122.60 \\
\hline .090 & $50 \%$ & 775.31 & 346.96 & 535.00 & 846.52 & 524.73 & 605.70 & 181.81 & 91.6 & 514.10 \\
\hline .040 & $75 \%$ & 893.00 & 1010.00 & 1080.00 & 864.00 & 1010.00 & 971.40 & 80.57 & 161.7 & 809.70 \\
\hline .090 & $75 \%$ & 845.99 & 788.16 & 1400.29 & 1417.21 & 1300.00 & 1150.33 & 275.64 & 161.7 & 988.63 \\
\hline .040 & $100 \%$ & 659.95 & 1048.18 & 925.23 & 1028.04 & 1165.67 & 965.41 & 170.76 & 252 & 713.41 \\
\hline .090 & $100 \%$ & 1394.73 & 1471.08 & 1336.54 & 1289.38 & 1337.31 & 1365.81 & 62.34 & 252 & 1113.81 \\
\hline
\end{tabular}


Table G.4 Al 6061 T6 burr thickness data at feed $.002 \mathrm{in} / \mathrm{rev}$

\begin{tabular}{|c|c|c|c|c|c|c|c|c|c|c|}
\hline $\begin{array}{l}\text { Material } \\
\text { thickness } \\
\text { [in] }\end{array}$ & $\begin{array}{l}\text { Level of } \\
\text { drill bit } \\
\text { wear }\end{array}$ & $\begin{array}{l}\text { Thickness } \\
1[\mu \mathrm{m}]\end{array}$ & $\begin{array}{l}\text { Thickness } \\
2[\mu \mathrm{m}]\end{array}$ & $\begin{array}{l}\text { Thickness } \\
3[\mu \mathrm{m}]\end{array}$ & $\begin{array}{l}\text { Thickness } \\
4[\mu \mathrm{m}]\end{array}$ & $\begin{array}{l}\text { Thickness } \\
5[\mu \mathrm{m}]\end{array}$ & $\begin{array}{l}\text { Mean } \\
\text { burr } \\
\text { thickness } \\
{[\mu \mathrm{m}]}\end{array}$ & $\begin{array}{l}\text { Standard } \\
\text { deviation }\end{array}$ & $\begin{array}{l}\text { Simulation } \\
\text { burr } \\
\text { thickness } \\
{[\mu \mathrm{m}]}\end{array}$ & $\begin{array}{l}\text { Delta between } \\
\text { simulation and } \\
\text { experimental } \\
\text { burr thickness } \\
{[\mu \mathrm{m}]}\end{array}$ \\
\hline .090 & $0 \%$ & 17.70 & 43.50 & 15.80 & 22.30 & 25.70 & 25.00 & 9.88 & 35.1 & -10.10 \\
\hline .040 & $25 \%$ & 25.50 & 26.30 & 15.50 & 14.10 & 32.30 & 22.74 & 6.91 & 44.1 & -21.36 \\
\hline .090 & $50 \%$ & 43.40 & 25.30 & 36.00 & 43.78 & 44.7 & 38.63 & 7.35 & 86.7 & -48.07 \\
\hline .090 & $75 \%$ & 28.50 & 35.70 & 35.50 & 32.80 & 34.70 & 33.44 & 2.67 & 175.5 & -142.06 \\
\hline .040 & $75 \%$ & 25.30 & 17.30 & 16.00 & 21.10 & 31.30 & 22.20 & 5.59 & 175.5 & -153.30 \\
\hline .040 & $100 \%$ & 15.30 & 23.10 & 35.30 & 35.00 & 36.30 & 29.00 & 8.38 & 286.1 & -257.10 \\
\hline .090 & $100 \%$ & 32.00 & 34.30 & 42.70 & 26.50 & 29.70 & 33.04 & 5.48 & 286.1 & -253.06 \\
\hline
\end{tabular}


Table G.5 Al 6061 T6 burr thickness data at feed $.004 \mathrm{in} / \mathrm{rev}$

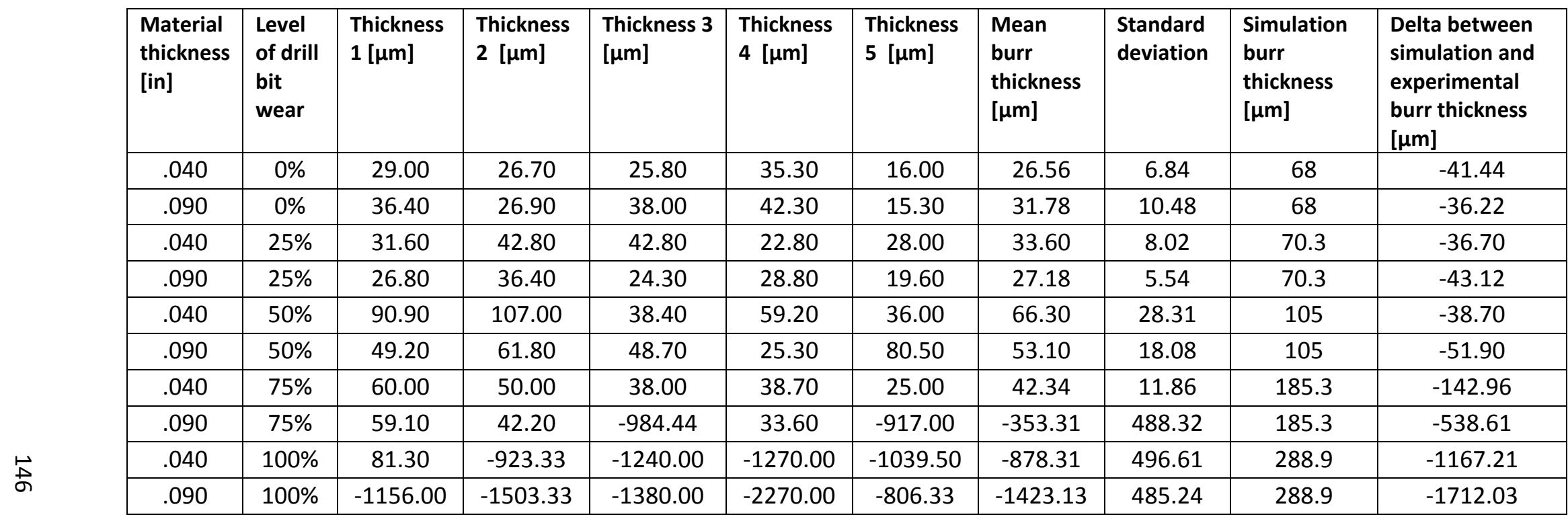


Table G.6 Al 6061 T6 burr thickness data at feed .006 in/rev

\begin{tabular}{|c|c|c|c|c|c|c|c|c|c|c|}
\hline $\begin{array}{l}\text { Material } \\
\text { thickness } \\
\text { [in] }\end{array}$ & $\begin{array}{l}\text { Level } \\
\text { of drill } \\
\text { bit } \\
\text { wear }\end{array}$ & $\begin{array}{l}\text { Thickness } \\
1[\mu \mathrm{m}]\end{array}$ & $\begin{array}{l}\text { Thickness } \\
2[\mu \mathrm{m}]\end{array}$ & $\begin{array}{l}\text { Thickness } \\
3[\mu \mathrm{m}]\end{array}$ & $\begin{array}{l}\text { Thickness } \\
4[\mu \mathrm{m}]\end{array}$ & $\begin{array}{l}\text { Thickness } \\
5[\mu \mathrm{m}]\end{array}$ & $\begin{array}{l}\text { Mean } \\
\text { burr } \\
\text { thickness } \\
{[\mu \mathrm{m}]}\end{array}$ & \begin{tabular}{|l|} 
Standard \\
deviation
\end{tabular} & \begin{tabular}{|l|} 
Simulation \\
burr \\
thickness \\
{$[\mu \mathrm{m}]$}
\end{tabular} & $\begin{array}{l}\text { Delta between } \\
\text { simulation and } \\
\text { experimental } \\
\text { burr thickness } \\
{[\mu \mathrm{m}]}\end{array}$ \\
\hline .090 & $0 \%$ & 44.50 & 36.70 & 62.70 & 21.80 & 31.70 & 39.48 & 13.75 & 99.3 & -59.82 \\
\hline .040 & $25 \%$ & 61.20 & 38.80 & 43.80 & 28.80 & 32.80 & 41.08 & 11.29 & 95.4 & -54.32 \\
\hline .090 & $50 \%$ & -1453.00 & 60.00 & 88.80 & 49.20 & 55.30 & -239.94 & 606.68 & 122.4 & -362.34 \\
\hline .040 & $75 \%$ & -1230.00 & -1280.00 & -1177.50 & -1360.00 & -1306.67 & -1270.83 & 62.76 & 194.7 & -1465.53 \\
\hline .090 & $75 \%$ & -1340.00 & -1310.00 & -1417.33 & -1520.00 & -1365.00 & -1390.47 & 73.72 & 194.7 & -1585.17 \\
\hline .040 & $100 \%$ & -740.00 & -1480.00 & -1642.00 & -1592.50 & -1548.00 & -1400.50 & 334.52 & 291.6 & -1692.10 \\
\hline .090 & $100 \%$ & -1556.00 & -1590.00 & -1536.67 & -1316.67 & -1405.00 & -1480.87 & 103.33 & 291.6 & -1772.47 \\
\hline
\end{tabular}


Table G.7 Al 7075 T6 burr height data at feed $.002 \mathrm{in} / \mathrm{rev}$

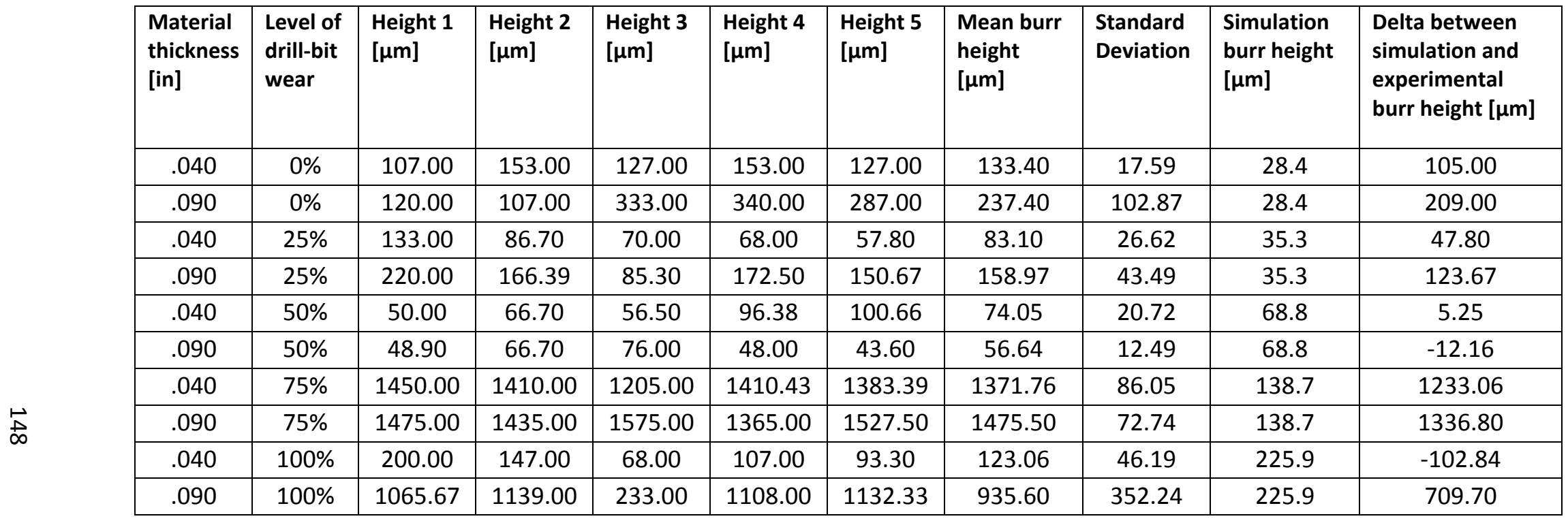


Table G.8 Al 7075 T6 burr height data at feed $.004 \mathrm{in} / \mathrm{rev}$

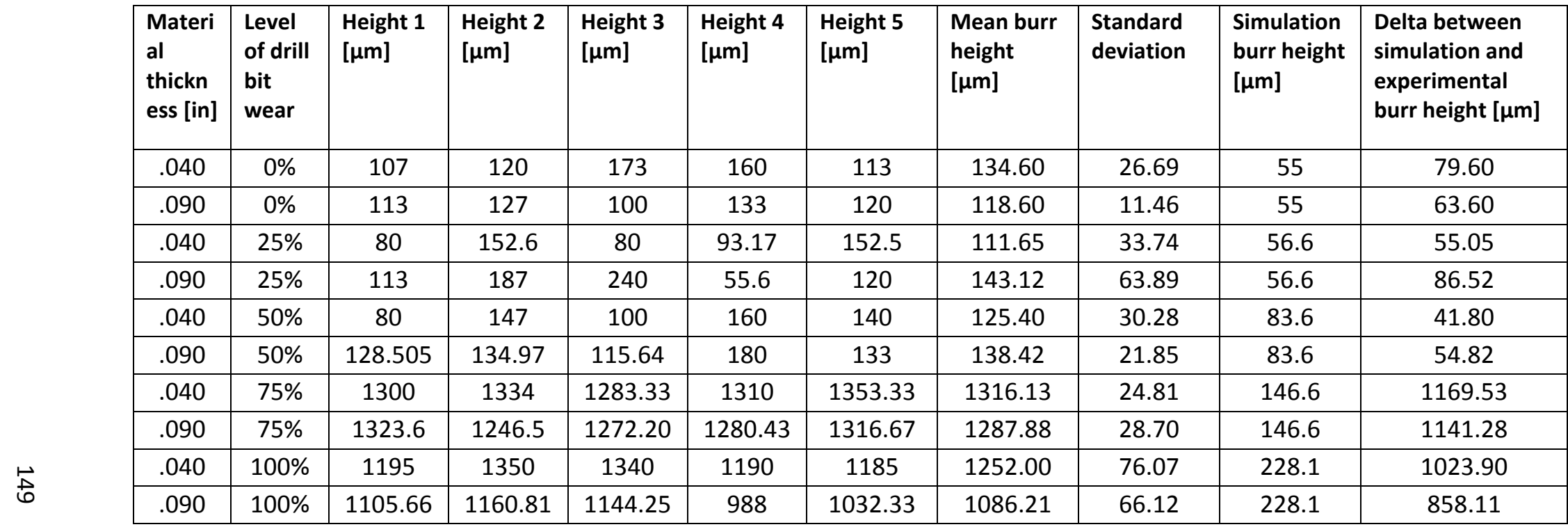


Table G.9 Al 7075 T6 burr height data at feed $.006 \mathrm{in} / \mathrm{rev}$

\begin{tabular}{|l|l|l|l|l|l|l|l|l|c|c|}
\hline $\begin{array}{l}\text { Material } \\
\text { thickness } \\
\text { [in] }\end{array}$ & $\begin{array}{l}\text { Level } \\
\text { of drill } \\
\text { bit } \\
\text { wear }\end{array}$ & $\begin{array}{l}\text { Height 1 } \\
{[\mu \mathrm{m}]}\end{array}$ & $\begin{array}{l}\text { Height 2 } \\
{[\mu \mathrm{m}]}\end{array}$ & $\begin{array}{l}\text { Height 3 } \\
{[\mu \mathrm{m}]}\end{array}$ & $\begin{array}{l}\text { Height 4 } \\
{[\mu \mathrm{m}]}\end{array}$ & $\begin{array}{l}\text { Height 5 } \\
{[\mu \mathrm{m}]}\end{array}$ & $\begin{array}{l}\text { Mean } \\
\text { burr } \\
\text { height } \\
{[\mu \mathrm{m}]}\end{array}$ & $\begin{array}{l}\text { Standard } \\
\text { deviation }\end{array}$ & $\begin{array}{l}\text { Simulation } \\
\text { burr height } \\
{[\mu \mathrm{m}]}\end{array}$ & $\begin{array}{l}\text { Delta between } \\
\text { simulation and } \\
\text { experimental } \\
\text { burr height } \\
{[\mu \mathrm{m}]}\end{array}$ \\
\hline .040 & $0 \%$ & 147 & 109 & 127 & 193 & 180 & 151 & 31.50 & 80.40 & 70.80 \\
\hline .090 & $0 \%$ & 160 & 140 & 100 & 167 & 143 & 142 & 23.32 & 80.40 & 61.60 \\
\hline .040 & $25 \%$ & 158.49 & 80 & 206.5 & 150.00 & 85 & 136 & 47.77 & 76.80 & 59.20 \\
\hline .090 & $25 \%$ & 280 & 86.7 & 236.5 & 320 & 147 & 214 & 85.78 & 76.80 & 137.24 \\
\hline .040 & $50 \%$ & 120 & 107 & 127 & 153 & 99.59 & 121 & 18.52 & 97.70 & 23.62 \\
\hline .090 & $50 \%$ & 102.80 & 99.59 & 93.30 & 166.67 & 120.00 & 116 & 26.61 & 97.70 & 18.77 \\
\hline .040 & $75 \%$ & 1137.67 & 1131 & 1173.33 & 1176.67 & 1206.67 & 1165 & 27.73 & 154.20 & 1010.87 \\
\hline .090 & $75 \%$ & 1300.06 & 1378.61 & 1260 & 1300 & 1305 & 1309 & 38.53 & 154.20 & 1154.53 \\
\hline .040 & $100 \%$ & 1255 & 1215 & 990.00 & 1011.50 & 1080 & 1110 & 106.82 & 230.20 & 880.10 \\
\hline .090 & $100 \%$ & 1173.33 & 1160.00 & 933.00 & 1199.99 & 1135.86 & 1120 & 95.98 & 230.20 & 890.24 \\
\hline
\end{tabular}


Table G.10 Al 7075 T6 burr thickness data at feed $.002 \mathrm{in} / \mathrm{rev}$

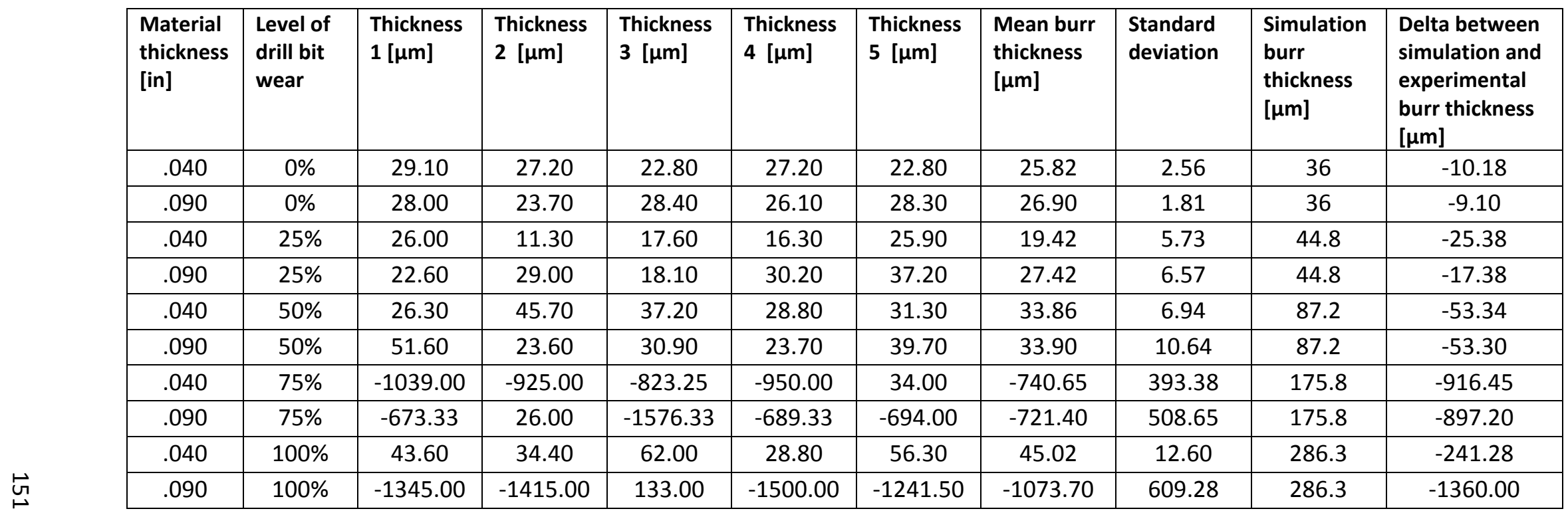


Table G.11 Al 7075 T6 burr thickness data at feed $.004 \mathrm{in} / \mathrm{rev}$

\begin{tabular}{|c|c|c|c|c|c|c|c|c|c|c|}
\hline $\begin{array}{l}\text { Material } \\
\text { thickness } \\
\text { [in] }\end{array}$ & $\begin{array}{l}\text { Level } \\
\text { of drill } \\
\text { bit } \\
\text { wear }\end{array}$ & $\begin{array}{l}\text { Thickness } \\
1[\mu \mathrm{m}]\end{array}$ & $\begin{array}{l}\text { Thickness } \\
2[\mu \mathrm{m}]\end{array}$ & $\begin{array}{l}\text { Thickness } \\
3[\mu \mathrm{m}]\end{array}$ & $\begin{array}{l}\text { Thickness } \\
4[\mu \mathrm{m}]\end{array}$ & $\begin{array}{l}\text { Thickness } \\
5[\mu \mathrm{m}]\end{array}$ & 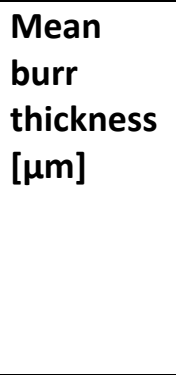 & $\begin{array}{l}\text { Standard } \\
\text { deviation }\end{array}$ & $\begin{array}{l}\text { Simulation } \\
\text { burr } \\
\text { thickness } \\
\text { [ } \mathrm{mm}]\end{array}$ & $\begin{array}{l}\text { Delta } \\
\text { between } \\
\text { simulation } \\
\text { and } \\
\text { experiment } \\
\text { al burr } \\
\text { thickness } \\
\text { [ } \mathrm{\mu m}]\end{array}$ \\
\hline \multirow{8}{*}{$\stackrel{\sim}{N}$} & $0 \%$ & 24 & 32.3 & 25.6 & 17.2 & 28.4 & 25.50 & 5.02 & 69.7 & -44.20 \\
\hline & $0 \%$ & 28.7 & 21.3 & 26.2 & 41.8 & 20.8 & 27.76 & 7.62 & 69.7 & -41.94 \\
\hline & $25 \%$ & 27.7 & 11.3 & 46.4 & 43.2 & 46 & 34.92 & 13.66 & 71.7 & -36.78 \\
\hline & $25 \%$ & 22.2 & 9.5 & 27.4 & 22.6 & 25 & 21.34 & 6.21 & 71.7 & -50.36 \\
\hline .040 & $50 \%$ & 60 & 41.4 & 32.7 & 20.3 & 35.6 & 38.00 & 12.98 & 105.9 & -67.90 \\
\hline .090 & $50 \%$ & 20.7 & 18.9 & 34.2 & 27.6 & 56.9 & 31.66 & 13.73 & 105.9 & -74.24 \\
\hline & $75 \%$ & -1291.67 & -1230.5 & -1223.33 & -1447.5 & -1255.00 & -1289.60 & 82.48 & 185.8 & -1475.40 \\
\hline & $75 \%$ & -1190 & -1170 & -1314 & -1186.67 & -1360.00 & -1244.13 & 77.50 & 185.8 & -1429.93 \\
\hline & $100 \%$ & -1642.5 & -1770 & -1693.33 & -1570 & -1612.5 & -1657.67 & 69.06 & 289 & -1946.67 \\
\hline & $100 \%$ & -1299.25 & -1190 & -1503.33 & -1363.33 & -1313.33 & -1333.85 & 101.89 & 289 & -1622.85 \\
\hline
\end{tabular}


Table G.12 Al 7075 T6 burr thickness data at feed $.006 \mathrm{in} / \mathrm{rev}$

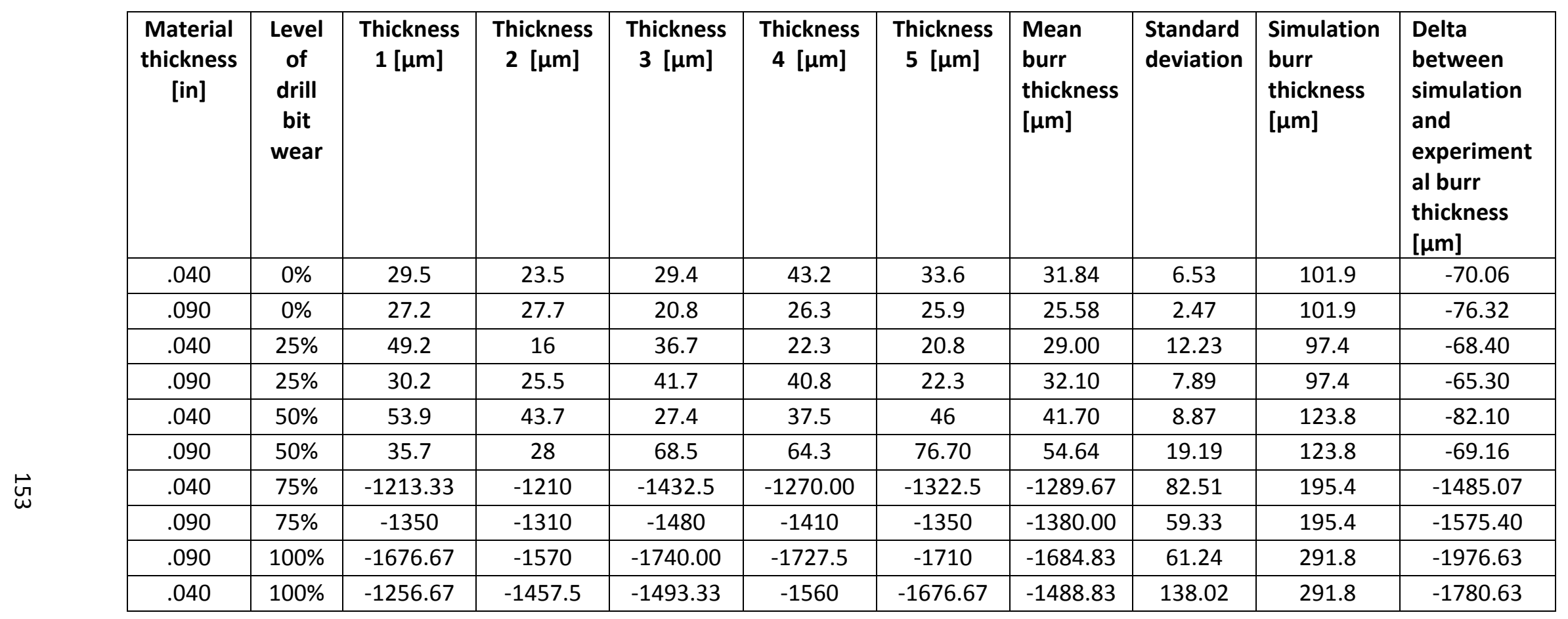




\section{BIBLIOGRAPHY}

[1] F. C. Campbell, Manufacturing Technology for Aerospace Structural Materials. San Diego, CA, USA: Elsevier, 2006.

[2] A. Cort, "Automating Wing Panel Assembly," Assembly Magazine, Jul. 2006.

[3] N. Jayaweera and P. Webb, "Automated assembly of fuselage skin panels," Assembly Automation, vol. 27, no. 4, pp. 343-355, 2007.

[4] F. Xi, "Advanced Aerospace Manufacturing Lecture Material." Ryerson University, Toronto, ON, Canada, 2008.

[5] J. Leopold and R. Wohlgemuth, "Modeling and Simulation of Burr Formation: State-of-the-Art and Future Trends," in Burrs - Analysis, Control and Removal, Heidelberg, Germany: Springer, 2010, pp. 79-86.

[6] L. K. Gillespie and P. T. Blotter, "The Formation and Properties of Machining Burrs," American Society of Mechanical Engineers Journal of Engineering for Industry, vol. 98, no. 1, pp. 66-74, 1976.

[7] L. K. Gillespie, Deburring and Edge Finishing Handbook, 1st ed. Dearborn, MI, USA: Society of Manufacturing Engineers, 1999.

[8] S. Basavarajappa, G. Chandramohan, M. Ashwin, M. Prabu, and K. Mukund, "Analysis of burr formation during drilling of hybrid metal matrix composites using design of experiments," International Journal of Machining and Machinability of Materials, vol. 1, no. 4, pp. 500, 2006.

[9] D. A. Davidson, "Surface condition impacts part performance," Metal Finishing, vol. 105, no. 2, pp. 22-31, 2007.

[10] D. J. Waldorf, "Shearing, ploughing and wear in orthogonal machining," Urbana, IL, USA: University of Illinois at Urbana-Champaign, 1996.

[11] L. K. Lauderbaugh, "Analysis of the effects of process parameters on exit burrs in drilling using a combined simulation and experimental approach," Journal of Materials Processing Technology, vol. 209, no. 4, pp. 1909-1919, 2009.

[12] J. Kim and D. A. Dornfeld, "Development of an analytical model for drilling burr formation in ductile materials," Journal of Engineering Materials and Technology, vol. 124, no. 2, pp. 192-198, 2002.

[13] S. S. Pande and H. P. Relekar, "Investigations on reducing burr formation in drilling," International Journal of Machine Tool Design and Research, vol. 26, no. 3, pp. 339-348, 1986.

[14] V. N. Gaitonde, S. R. Karnik, B. T. Achyutha, B. Siddeswarappa, and J. P. Davim, "Predicting burr size in drilling of AISI 316L stainless steel using response surface analysis," International Journal of Materials and Product Technology, vol. 35, no. 1-2, pp. 228-245, 2009.

[15] D. J. Waldorf, R. E. DeVor, and S. G. Kapoor, "A slip-line field for ploughing during orthogonal cutting," Journal of Manufacturing Science and Engineering, vol. 120, no. 4, pp. 693, 1998. 
[16] L. K. Gillespie, "Your burr technology efforts changed the world," in Proceedings of the 8th International Deburring and Surface Finishing Conference, Osaka, Japan, 2006, pp. 1-37.

[17] L. K. Gillespie, "The formation and properties of machining burrs," Logan, UT, USA: Utah State University, 1973.

[18] L. K. L. Saunders and C. A. Mauch, "An exit burr model for drilling of metals," Journal of Manufacturing Science and Engineering, vol. 123, no. 4, pp. 562-566, 2001.

[19] M.-F. Huang and T.-R. Lin, "Application of grey-Taguchi method to optimise drilling of aluminium alloy 6061 with multiple performance characteristics," Materials Science and Technology, vol. 20, no. 4, pp. 528-532, 2004.

[20] N. Tosun, "Determination of optimum parameters for multi-performance characteristics in drilling by using grey relational analysis," International Journal of Advanced Manufacturing Technology, vol. 28, no. 5-6, pp. 450-455, 2006.

[21] E. Brinksmeier and S. Fangmann, "Burr and cap formation by orbital drilling of aluminum," in Burrs - Analysis, Control and Removal, Heidelberg, Germany: Springer, 2010, pp. 31-45.

[22] F. Z. Fang, K. Liu, T. R. Kufess, and Lim G.C., "Tool-based micro machining and applications in," in MEMS/NEMS Handbook Techniques and Applications Vol. 3 Manufacturing Methods, C. T. Leondes, Ed. Los Angeles, CA, USA: Springer, 2006, p. 86.

[23] D. A. Dornfeld, J. S. Kim, H. Dechow, J. Hewson, and L. J. Chen, "Drilling burr formation in titanium alloy, Ti-6AI-4V," CIRP Annals - Manufacturing Technology, vol. 48, no. 1, pp. 73-76, 1999.

[24] A. Sofronas, "The formation and control of drilling burrs," Detroit, MI, USA: University of Detroit, 1975.

[25] T. Matsumura, J. Leopold, and S.-V. B. Heidelberg, "Cutting force model for analysis of burr formation in drilling process," in Burrs - Analysis, Control and Removal, Heidelberg, Germany: Springer, 2010, pp. 47-53.

[26] J. Kim and D. A. Dornfeld, "Development of a drilling burr control chart for stainless steel," Transactions of the North American Manufacturing Research Institute of the Society of Manufacturing Engineers, vol. 28, pp. 317-322, 2000.

[27] R. Furness, "High speed video of drilling burr formation." AMTD, Ford Motor Company, 1998.

[28] L. Leitz, J. C. Aurich, and V. Franke, "Burr formation in drilling intersecting holes," in Burrs Analysis, Control and Removal, Heidelberg, Germany: Springer, 2010, pp. 99-105.

[29] D. A. Stephenson and J. S. Agapiou, Metal Cutting Theory and Practice, 3rd ed. Boca Raton, FL, USA: CRC Press, 2016.

[30] L. Liao, F. Xi, and S. Engin, "Robotic deburring based on on-line burr measurement," in Burrs Analysis, Control and Removal, Heidelberg, Germany: Springer, 2010, pp. 213-220.

[31] M. P. Groover, Fundamentals of Modern Manufacturing: Materials, Processes, and Systems, 2nd ed. New York, NY: John Wiley \& Sons Inc., 2002. 
[32] A. M. Abdelhafeez, S. L. Soo, D. K. Aspinwall, A. Dowson, and D. Arnold, "Burr formation and hole quality when drilling titanium and aluminium alloys," Procedia CIRP, 2015, vol. 37, pp. 230-235.

[33] J. Wang, C. Z. Huang, and W. G. Song, "The effect of tool flank wear on the orthogonal cutting process and its practical implications," Journal of Materials Processing Technology, vol. 142, no. 2, pp. 338-346, 2003.

[34] Y. Altintas, "Tool Wear and Tool Breakage," in Manufacturing Automation - Metal Cutting Mechanics, Machine Tool Vibrations, and CNC Design, 2nd ed., New York, NY, USA: Cambridge University Press, 2012, pp. 54-62.

[35] A. Faraz, D. Biermann, and K. Weinert, "Cutting edge rounding : An innovative tool wear criterion in drilling CFRP composite laminates," International Journal of Machine Tools \& Manufacture, vol. 49, no. 15, pp. 1185-1196, 2009.

[36] B. Luo, K. Zhang, Y. Li, H. Cheng, and S. Liu, "Modelling of thrust force for worn drill bits characterised by cutting edge radius in drilling carbon fibre-reinforced plastic/Ti-6Al-4V alloy stacks," Proceedings of Institution of Mechanical Engineering Part B: Journal of Engineering Manufacture, vol. 232, no. 11, pp. 1960-1972, 2018.

[37] P. Dewhurst and I. F. Collins, "A matrix technique for constructing slip-line field solutions to a class of plane strain plasticity problems," International Journal for Numerical Methods in Engineering, vol. 7, no. 3, pp. 357-378, 1973.

[38] J. Leopold, G. Schmidt, K. Hoyer, and A. Freitag, "Modeling and Simulation of Burr Formation State-of-the-Art and Future Trends," in 8th CIRP International Workshop on Modeling and Machining Operations, 2005, Chemnitz, Germany, pp. 73-83.

[39] A. J. Black, E. M. Kopalinsky, and P. L. B. Oxley, "Asperity deformation models for explaining the mechanisms involved in metallic sliding friction and wear - a review,"Proceedings of the Institution of Mechanical Engineers, Part C: Journal of Mechanical Engineering Science, vol. 207, no. 5, p. 335-353, 1993.

[40] T. Shi and S. Ramalingam, "Slip-line solution for orthogonal cutting with a chip breaker and flank wear," International Journal of Mechanical Sciences, vol. 33, no. 9, pp. 689-704, 1991.

[41] S. Chinchanikar and S. K. Choudhury, "Cutting force modeling considering tool wear effect during turning of hardened AISI 4340 alloy steel using multi-layer TiCN/AI2O3/TiN-coated carbide tools," International Journal of Advanced Manufacturing Technology, vol. 83, no. 9-12, pp. 1749-1762, 2016.

[42] Y. Long and Y. Huang, "Combined effects of flank and crater wear on cutting force modeling in orthogonal machining part I: Model development," Machining Science and Technology, vol. 14, no. 1, pp. 1-23, 2010.

[43] Y. Long, Y. Huang, and X. Sun, "Combined effects off flank and crater wear on cutting force modeling in orthogonal machining-part II: Bayesian approach-based model validation," Machining Science and Technology, vol. 14, no. 1, pp. 24-42, 2010.

[44] E. Kilickap, "Modeling and optimization of burr height in drilling of Al-7075 using Taguchi method and response surface methodology," International Journal of Advanced Manufacturing Technology., vol. 49, no. 9-12, pp. 911-923, 2010. 
[45] N. Feito, A. S. Milani, and A. Muñoz-Sánchez, "Drilling optimization of woven CFRP laminates under different tool wear conditions: A multi-objective design of experiments approach," Structural and Multidisciplinary Optimization, vol. 53, no. 2, pp. 239-251, 2016.

[46] B. Avitzur, C. K. Huang, and Y. D. Zhu, "A friction model based on the upper-bound approach to the ridge and sublayer deformations," Wear, vol. 95, no. 1, pp. 59-77, 1984.

[47] M. Abebe and F. C. Appl, "A slip-line solution for negative rake angle cutting," in Proceedings of the 9th North American Manufacturing Research Conference, 1981, pp. 341-348.

[48] ASM, ASM Handbook Vol. 3, Aluminum Fabrication and Finishing. Metals Park, OH, USA: American Society for Metals, 1967.

[49] ASM, ASM Handbook Vol. 2, Properties and Selection: Nonferrous Alloys and Special-Purpose Materials. Metals Park, OH, USA: ASM International, 1992.

[50] ASM, "Machining of Aluminum and Aluminum Alloys," in ASM Handbook Vol. 16, Machining, 9th ed., Materials Park, OH, USA: ASM International, 1989, pp. 761-765.

[51] Clarkson-Osborn Tools Limited, "HSS \& Carbide Metal Cutting Tools Catalogue.", Brampton, ON, Canada: Clarkson-Osborn Tools Ltd., 2014.

[52] Federal Aviation Administration, Advisory Circular. Washington, DC, USA: Federal Aviation Administration, 2011, p. 7.

[53] F. Schäfer, Entgraten. Mainz: Krausskopfverlag, 1975.

[54] V. Franke, L. Leitz, and J. C. Aurich, "Burr measurement: A round robin test comparing different methods," in Burrs - Analysis, Control and Removal, Heidelberg, Germany: Springer, 2010, pp.167-178.

[55] Khan Academy, "Population and sample standard deviation review (article) | Khan Academy," 2018. [Online]. Available: https://www.khanacademy.org/math/statisticsprobability/summarizing-quantitative-data/variance-standard-deviation-sample/a/populationand-sample-standard-deviation-review. [Accessed April 20, 2019].

[56] Y. B. Guo and Y. K. Chou, "The determination of ploughing force and its influence on material properties in metal cutting," Journal of Materials Processing Technology, vol. 148, no. 3, pp. 368375, 2004.

[57] "Ductility Review - Strength Mechanics of Materials - Engineers Edge," Engineeredge, 2018. [Online]. Available: https://www.engineersedge.com/material_science/ductility.htm. [Accessed July 19, 2018]. 